\title{
IN-FLIGHT RECEPTIVITY EXPERIMENTS ON A 30-DEGREE SWEPT-WING USING MICRON-SIZED DISCRETE ROUGHNESS ELEMENTS
}

\author{
A Dissertation \\ by \\ ANDREW LEE CARPENTER \\ Submitted to the Office of Graduate Studies of \\ Texas A\&M University \\ in partial fulfillment of the requirements for the degree of \\ DOCTOR OF PHILOSOPHY
}

May 2009

Major Subject: Aerospace Engineering 


\title{
IN-FLIGHT RECEPTIVITY EXPERIMENTS ON A 30-DEGREE SWEPT-WING USING MICRON-SIZED DISCRETE ROUGHNESS ELEMENTS
}

\author{
A Dissertation \\ by \\ ANDREW LEE CARPENTER \\ Submitted to the Office of Graduate Studies of \\ Texas A\&M University \\ in partial fulfillment of the requirements for the degree of \\ DOCTOR OF PHILOSOPHY
}

\begin{abstract}
Approved by:
Chair of Committee, William Saric

Committee Members, Helen Reed

Donald Ward

Andrew Duggleby

Head of Department, Dimitris Lagoudas
\end{abstract}

May 2009

Major Subject: Aerospace Engineering 


\begin{abstract}
In-flight Receptivity Experiments on a 30-degree Swept-wing Using Micron-sized

Discrete Roughness Elements. (May 2009)

Andrew Lee Carpenter, B.S., Arizona State University

Chair of Advisory Committee: Dr. William Saric
\end{abstract}

One of the last remaining challenges preventing the laminarization of sweptwings is the control of unstable crossflow vortices. In low-disturbance environments the transition from laminar to turbulent flow on the swept-wing initially takes the path of receptivity, where surface roughness or disturbances in the environment introduce shortwavelength disturbances into the boundary layer. This is followed by development and linear growth of stationary crossflow vortices that modify the mean flow, changing the stability characteristics of the boundary layer. Finally, breakdown to turbulence occurs over a short length scale due to the high-frequency secondary instability. The receptivity mechanism is the least understood, yet holds the most promise for providing a laminar flow control strategy. Results of a 3-year flight test program focused on receptivity measurements and laminar flow control on a 30-degree swept-wing are presented. A swept-wing test article was mounted on the port wing of a Cessna O-2A aircraft and operated at a chord Reynolds number of 6.5 to 7.5 million. Spanwise-periodic, micronsized discrete roughness elements were applied at the leading edge of the swept-wing in order to excite the most unstable crossflow wavelength and promote early boundary- 
layer transition. An infrared camera was used to detect boundary-layer transition due to changes in leading-edge roughness. Combined with the IR camera, a new technique of calibrating surface-mounted hotfilms was developed for making disturbance-amplitude measurements downstream of modulated roughness heights. This technique proved to be effective at measuring disturbance amplitudes and can be applied in future tests where instrumentation is limited. Furthermore, laminar flow control was performed with subcritically-spaced roughness. A 100\% increase in the region of laminar flow was achieved for some of the conditions tested here. 


\section{DEDICATION}

I would like to dedicate this work to my wife and best friend, Angela, who was willing to pick up everything and move to College Station so I could pursue this research. Her support during this time is greatly appreciated and I am forever indebted to her because of this. I would also like to dedicate this work to my Father, who initially sparked my interest in aviation. 


\section{ACKNOWLEDGEMENTS}

First and foremost I would like to thank my committee chair, Dr. William Saric, for recruiting me first as an undergraduate and then supporting me as a graduate student. It has been a long journey, but I thank him for giving me responsibility to make difficult decisions and then learn from my mistakes. I am a better engineer because of this. $\mathrm{He}$ has also instilled in me hard-work ethics and a demand for perfection that I will carry with me for my lifetime. Thank you.

I would also like to thank my committee members. Dr. Helen Reed provided technical support and stability calculations, and always had a sense of support and good spirit even when none of the data made sense. I am forever indebted to Dr. Donald Ward for sharing his experiences and expertise in the field of flight testing. I am a safer engineer because of you. Thank you, Dr. Andrew Duggleby for serving on my committee on such short notice.

I am grateful to the pilots. Without them, none of this would be possible. Roy Martin helped get this experiment up and running and provided most of the flying time in the beginning. We would still be in front of the Safety Review Board if both Roy Martin and Dr. Donald Ward had not been our pilots. Thanks to Celine Kluzek who helped form the Flight Research Laboratory and always had a sense of safety and good 
spirit. I am also grateful to Lee Denham who stepped up in the end to help me finish these experiments.

I would like to thank all past, present, and future students of the Texas A\&M Flight Research Laboratory. They were with me during every step of the experiments. Without them showing up seven days a week, twelve hours a day, this would not be possible. Specifically I am grateful for Lauren Hunt, who always provided constructive criticism and served as my technical back-up, Shane Schouten for his strong work ethic, Jerrod Hofferth for always being available during early morning flights and weekends, Rick Rhodes and Tyler Neale for providing computations for these experiments, and lastly Brian Crawford and Matt Woodruff who will continue with this research.

I would like to thank Mr. Cecil Rhodes for showing me the way of the A\&P mechanic. I will truly miss our conversations on airplanes, inventions, and anything engineering related. Lastly, I would like to thank Ms. Colleen Leatherman. The Flight Research Laboratory and Unsteady Wind Tunnel would not operate without you. I will miss you most. 


\section{NOMENCLATURE}

A

AoA

$C_{f}$

$a, b, c, d$

$d$

$d / k$

$k$

KIAS

KTAS

m

$N$

n

$T u$

$l$

$l / d$

$U$

V

W

$\alpha$

$\beta$

$\delta$
Empirical constant for hotfilm calibration equation

SWIFT model angle of attack

Skin-friction coefficient

Least squares polynomial curve fit constants

Diameter of roughness element

Ratio of roughness diameter to roughness height

Roughness height

Indicated airspeed in knots

True airspeed in knots

Hotfilm temperature drift constant

$N$-factor

Emperical constant for hotfilm calibration equation

Streamwise freestream turbulence intensity

Spacing between two roughness elements, not center to center

Ratio of roughness spacing to roughness diameter

Freestream $X$-velocity

Freestream $Y$-velocity

Freestream Z-velocity

Aircraft angle of attack

Aircraft sideslip angle

Boundary layer height when $u$ is $99 \%$ of the edge velocity 


\begin{tabular}{|c|c|}
\hline$k / \delta$ & Ratio of roughness height to boundary layer height \\
\hline$\Delta p$ & Differential pressure measured by Preston tube \\
\hline$\Delta p^{+}$ & Non-dimensional Preston tube differential pressure \\
\hline$\lambda$ & Roughness element spacing \\
\hline$d / \lambda$ & Ratio of roughness diameter to roughness spacing \\
\hline$v$ & Freestream kinematic viscosity \\
\hline$\rho$ & Freestream air density \\
\hline$\tau^{+}$ & Non-dimensional wall-shear stress \\
\hline$d_{\text {prest }}$ & Outer diameter of Preston tube \\
\hline$E_{0}$ & Hotfilm voltage with zero airflow \\
\hline$E_{\text {comp }}$ & Temperature-compensated hotfilm voltage \\
\hline$E_{\text {measured }}$ & Measured hotfilm voltage \\
\hline$P_{a}$ & Mean of absolute deviations \\
\hline$P_{q}$ & RMS surface roughness \\
\hline$P_{y}$ & Maximum peak-to-peak value \\
\hline$P_{z}$ & Five-point average of maximum peak-to-peak values \\
\hline$R e_{c}$ & Chord Reynolds number \\
\hline$R e_{k}$ & Roughness Reynolds number based on $k$ \\
\hline $\operatorname{Re}_{k, t}$ & Critical $R e_{k}$ resulting in a boundary-layer trip \\
\hline$T_{\text {nom }}$ & Nominal temperature used for hotfilm temperature compensation \\
\hline$T_{s}$ & Surface temperature \\
\hline$u^{\prime}$ & Fluctuating component of $U$ \\
\hline
\end{tabular}




\begin{tabular}{|c|c|}
\hline$u_{k}$ & Absolute velocity at the top of the roughness element \\
\hline$u_{s}$ & Velocity tangential to swept-wing streamline \\
\hline$u_{t}$ & Velocity normal to swept-wing leading edge \\
\hline$v_{s}$ & Wall-normal velocity at the local streamline location \\
\hline$v_{t}$ & Wall-normal velocity at swept-wing leading edge \\
\hline$V_{n e}$ & Never-exceed speed \\
\hline$V_{\text {no }}$ & Top speed in turbulent conditions \\
\hline$w_{s}$ & Velocity normal to swept-wing streamline \\
\hline$w_{t}$ & Spanwise velocity parallel to swept-wing leading edge \\
\hline$z_{s}$ & Spanwise distance along the swept leading edge \\
\hline$\alpha_{t}$ & Streamwise wavenumber, tangential to swept-wing streamline \\
\hline$\alpha_{t o}$ & First calculated streamwise growth rate \\
\hline$\beta_{t}$ & Spanwise wavenumber, normal to streamline \\
\hline$\lambda_{\text {crit }}$ & Most unstable stationary crossflow wavelength \\
\hline$\Lambda_{L E}$ & Leading-edge sweep \\
\hline$v_{k}$ & Kinematic viscosity at the top of the roughness element \\
\hline$\tau_{w}$ & Wall-shear stress \\
\hline$\omega$ & Non-dimensional frequency \\
\hline
\end{tabular}


TABLE OF CONTENTS

Page

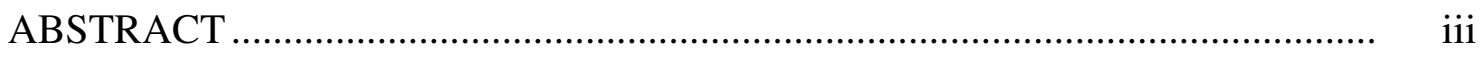

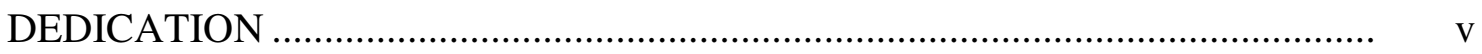

ACKNOWLEDGEMENTS ....................................................................... vi

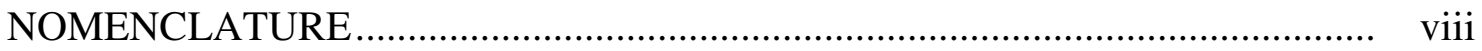

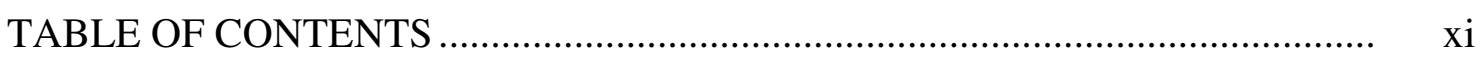

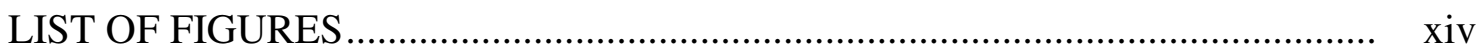

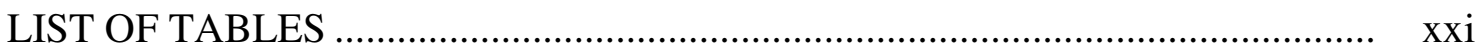

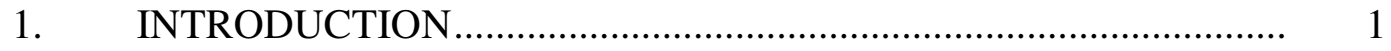

1.1 Overview and Motivation................................................................ 1

1.2 Crossflow Dominated Transition .................................................. 3

1.3 Experimental Objectives ............................................................... 8

2. EXPERIMENTAL SETUP ........................................................ 10

2.1 Experimental Requirements ........................................................ 10

2.2 Test Aircraft ......................................................................... 13

2.3 Swept-Wing In-Flight Testing (SWIFT) Model ........................... 17

2.4 Aircraft Configuration and Coordinate System ............................. 20

2.5 Safety Analysis...................................................................... 26

2.6 Flight Restrictions ..................................................................... 28

2.7 Flight Instrumentation ............................................................ $\quad 30$

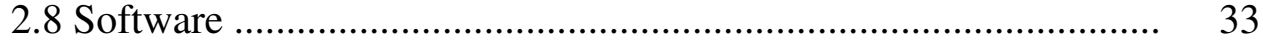

2.9 Freestream Measurements........................................................... 35

2.10 Measurement Uncertainty ........................................................... 40

2.11 Ground Based Instrumentation..................................................... 42

2.11.1 Surface Roughness Measurements ..................................... 42

2.11.2 Roughness Height Measurements ..................................... 46

2.12 Airport Operations.................................................................... 47 
2.13 Flight Number Documentation.............................................. 48

3. TEST CONDITIONS ............................................................ 49

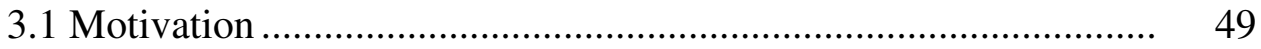

3.2 Turbulence Test Procedures ................................................... 50

3.3 Freestream Disturbance Results .............................................. 53

3.4 SWIFT Accelerometer Data .................................................. 53

3.5 Sound Pressure Levels ........................................................... 56

4. $\quad$ $\quad$ p MEASUREMENTS ............................................................ 57

4.1 Cp Measurement Procedures .................................................... 58

4.2 Comparison with CFD .......................................................... 65

4.3 Root and Tip Pressure Port Comparison .................................. 69

5. BASELINE INFARED THERMOGRAPHY ............................... 72

5.1 Infrared Thermography Overview......................................... 72

5.2 IR Camera Specifications ........................................................ 72

5.3 IR Thermography Procedures ................................................ 74

5.4 Atmospheric Variables ............................................................ 80

5.5 Polished Leading Edge IR Results ........................................... 86

5.6 Crossflow Excitation ............................................................... 92

5.7 Painted Leading Edge IR Results .......................................... 93

5.8 Baseline IR Comparison........................................................ 98

6. CROSSFLOW EXCITATION WITH CRITICAL DREs ................ 100

6.1 Literature Review ................................................................ 100

6.2 Stability Calculations ....................................................... 102

6.2.1 Linear Stability Theory ................................................. 102

6.2.2 Nonlinear Parabolized Stability Equations ........................ 107

6.3 Transition Results with Appliqué Critically Spaced DREs .......... 108

6.4 Effect of Roughness Diameter ................................................. 118

6.5 Design of Variable Height Roughness Elements ....................... 119

6.6 Transition Results with Variable Height Critical DREs .............. 124

6.7 Transition Results with Variable Height and Appliqué DREs...... 131

6.8 Comparison with Historical Roughness Tests ............................ 136 
7. RECEPTIVITY MEASUREMENTS ....................................... 140

7.1 Hotfilm Array Design ........................................................... 141

7.2 Preston Tube.......................................................................... 146

7.3 Temperature Compensation ................................................... 149

7.4 Hotfilm Calibration ........................................................... 155

7.5 Receptivity Results............................................................ 157

8. SWEPT-WING LAMINAR FLOW CONTROL WITH DREs.......... 164

8.1 Linear Stability Theory Calculations ........................................ 165

8.2 Appliqué DREs Forward of Critical Neutral Point ..................... 168

8.3 Variable Height DREs......................................................... 170

8.4 Appliqué DREs at the Critical Neutral Point ............................ 175

8.5 Appliqué DREs at the Control Neutral Point .............................. 177

8.6 Swept Wing Laminar Flow Control Summary .......................... 183

9. OPERATIONAL RECOMMENDATIONS .............................. 186

9.1 Insect Contamination............................................................. 187

9.2 Effect on Laminar Flow ....................................................... 192

10. CONCLUSIONS ................................................................. 197

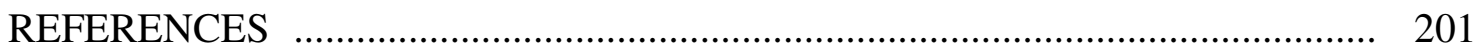

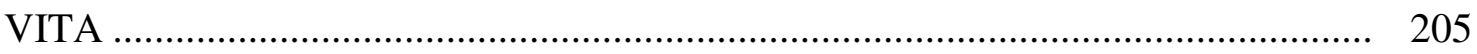




\section{LIST OF FIGURES}

FIGURE

Page

1 Inviscid streamline over a swept wing and standard coordinates used to describe swept wings

2 Swept-wing boundary layer profile showing the tangential, crossflow, and total velocity profiles. Figure courtesy of Saric et al. (2003).....

3 Development of stationary crossflow waves in the chordwise direction.

Figure adapted from Reibert et al. (1996).....

4 Unmodified test aircraft with dimensioned three-view drawing....

5 Breakaway view of the major SWIFT components showing the black powder-coat testside of the model.

6 Schematic of SWIFT model with five-hole probe and the hotwire sting...

7 Pictures of SWIFT model with five-hole probe and the hotwire sting ......

$8 \quad$ Freestream coordinate system for velocities and angles $\alpha$ and $\beta \ldots \ldots \ldots \ldots . .$.

9 Comparison of $A o A$ for the starboard and port outboard pylon locations..

10 Five-hole probe mounted to SWIFT model

11 Plots of $\alpha$ and $\beta$ showing both a smooth and a rough test point for the same dive at two different altitudes.

12 Picture of surface roughness measurements on the SWIFT leading edge .

13 Hotwire sting mounted to the port outboard pylon and air data boom mounted to the starboard outboard pylon.

14 Accelerometer power spectra for the ground rap test

15 Accelerometer power spectra recorded in flight in a level, cruise attitude 
FIGURE Page

16 Accelerometer power spectra in the dive attitude .............................. 56

17 Schematic of SWIFT model showing both root and tip pressure port rows

18 Infrared image showing two turbulent wedges produced by the leadingedge pressure ports and one wedge produced by an isolated roughness element

$19 C p$ data for three representative test points at the root pressure port row .. $\quad 62$

$20 C p$ data for three representative test points at the tip pressure port row ....

21 Experimental and computational comparison at -2.61 degrees $A o A$ for the root pressure port row.....

22 Experimental and computational comparison at -2.61 degrees $A o A$ for the tip pressure port row

23 Experimental and computational comparison at -4.69 degrees $A o A$ for the root pressure port row.

24 Experimental and computational comparison at -4.69 degrees $A o A$ for the tip pressure port row.....

25 Root and tip pressure port comparison for Test Point 31

70

26 Root and tip pressure port comparison for Test Point 27.

27 Plot of both laminar and turbulent surface temperatures and freestream total temperature during a dive producing good IR images

28 IR images showing radiation spots and markers on the model

29 Total temperature lapse-rate examples recorded throughout the year

30 Plot of both laminar and turbulent surface temperatures and freestream total temperature during a dive with a temperature inversion

31 Baseline IR image with the polished leading edge at -0.65 degrees $A o A$.. 
FIGURE

Page

32 Baseline IR image with the polished leading edge at -1.88 degrees $A o A$...

88

33 Baseline IR image with the polished leading edge at -3.17 degrees $A \circ A \ldots$

88

34 Baseline IR image with the polished leading edge at -4.39 degrees $A o A$...

35 Baseline IR image with the painted leading edge at -3.55 degrees $A o A \ldots .$.

36 Baseline IR image with the painted leading edge at -3.96 degrees $A o A \ldots .$.

37 Baseline IR image with the painted leading edge at -4.61 degrees AoA.... 96

38 Baseline IR image with the painted leading edge at -5.07 degrees $A o A \ldots .$.

39 Plot of transition Reynolds number for various AoAs and leading-edge roughness conditions

$40 N$-factors for Test Point 31 at -2.61 degrees $A o A$ along the root pressure port row

$41 \quad N$-factors for Test Point 31 at -2.61 degrees $A o A$ along the tip pressure port row

$42 \mathrm{~N}$-factors for Test Point 27 at -4.69 degrees $A o A$ along the root pressure port row

$43 N$-factors for Test Point 27 at -4.69 degrees $A o A$ along the tip pressure port row

44 Disturbance amplitudes for Test Point 31 at -2.61 degrees $A o A$ along the root pressure port row 108

45 Baseline IR image used to demonstrate critically-spaced DREs 110

46 Depiction of DREs applied to the leading edge with a different $x / \mathrm{c}$ location at the root and tip pressure port rows

47 Plot of root and tip boundary-layer heights for Test Point 27.

48 IR image with [24|4.5|1.1] (Root $R e_{k}=8.8, \operatorname{Tip} R e_{k}=10.1$, $\left.k / \delta_{\text {root }}=0.08, k / \delta_{\text {tip }}=0.08\right)$ critically-spaced DREs 
49 IR image with [30|4.5|1.1] (Root $\operatorname{Re}_{k}=14.1$, Tip $R e_{k}=16.3$, $\left.k / \delta_{\text {root }}=0.10, k / \delta_{\text {tip }}=0.10\right)$ critical DREs and $42 \mu \mathrm{m}\left(\operatorname{Root} R e_{k}=28.4\right.$, Tip $\left.R e_{k}=33.1, k / \delta_{\text {root }}=0.13, k / \delta_{\text {tip }}=0.14\right)$ isolated roughness elements in between the critically-spaced DREs.

50 IR image with [30|4.5|1.1] ( $\operatorname{Root} \operatorname{Re}_{\mathrm{k}}=14.1$, Tip $R e_{k}=16.3$, $\left.k / \delta_{\text {root }}=0.10, k / \delta_{\text {tip }}=0.10\right)$ critically-spaced DREs

51 IR image of the climb with [30|4.5|1.1] (Root $R e_{k}=14.1$, Tip $\left.R e_{k}=16.3, k / \delta_{\text {root }}=0.10, k / \delta_{\text {tip }}=0.10\right)$ critically-spaced DREs

52 Schematic of DRE insert assembly

53 Example of variable-height DRE calibration curves measured at the center of each roughness element......

54 Baseline IR image with polished leading edge and DRE insert with no pressure applied.

55 IR image with [31|4.5|1.1] (Root $R e_{k}=15.1$, Tip $R e_{k}=17.4$, $\left.k / \delta_{\text {root }}=0.10, k / \delta_{\text {tip }}=0.10\right)$ critically-spaced, variable-height DREs

56 IR image with [75|4.5|1.1] (Root $R e_{k}=93.4$, Tip $R e_{k}=109.8$, $\left.k / \delta_{\text {root }}=0.24, k / \delta_{\text {tip }}=0.24\right)$ critically-spaced, variable-height DREs

57 Schematic of $2 \mathrm{~mm}$ diameter, variable-height DREs and an appliqué DRE with a diameter of $1.1 \mathrm{~mm}$

58 Schematic of a variable-height DRE and an appliqué DRE indicating the effective diameter theory 130

59 Variable-height DRE and appliqué DRE scans at 0 psid applied pressure 132

60 Variable-height DRE and appliqué DRE scans at 2 psid applied pressure 133

61 Variable-height DRE and appliqué DRE scans at -2 psid applied pressure 133

$62 k=6 \mu \mathrm{m}\left(\operatorname{Root} R e_{k}=0.3\right.$, Tip $\left.R e_{k}=0.3, k / \delta_{\text {root }}=0.20, k / \delta_{\text {tip }}=0.20\right)$, $d=1.1 \mathrm{~mm}$ appliqué DREs on $2 \mathrm{~mm}$ variable-height DREs at 0 psid....... 
$63 k=6 \mu \mathrm{m}, d=1.1 \mathrm{~mm}$ appliqué DREs on $2 \mathrm{~mm}$ variable-height DREs with the variable-height DREs at $77 \mu \mathrm{m}$. Total roughness height $=83$ $\mu \mathrm{m}\left(\operatorname{Root} R e_{k}=115, \operatorname{Tip} R e_{k}=135, k / \delta_{\text {root }}=0.26, k / \delta_{\text {tip }}=0.27\right)$.

64 Plot showing reduction in $R e_{k, t}$ as two cylindrical roughness elements move close to each other. Figure from Carmichael (1958)

65 Custom hotfilm design for measuring disturbance amplitudes and crossflow vortex spacing.

66 Schematic of hotfilm, Preston tube, surface-mounted thermocouple, and static pressure port on the SWIFT model

$67 E_{o}$ measurements recorded in the FRL hangar

68 Filtered Preston tube data during the cold soak, dive, and hot soak

69 Wall shear stress and recorded hotfilm voltages during the dive 152

70 Example of finalized calibration curves after applying temperature compensation

71 Example calibration curve for one of the hotfilm sensors 157

72 IR image showing a laminar boundary layer over the hotfilm, Preston tube, static pressure port, and surface-mounted thermocouple .....

73 IR image of the hotfilm located just upstream of transition 159

74 IR image of the hotfilm located in a stable laminar boundary layer. 160

75 Power Spectral Density plot for hotfilm \#2 at two different AoAs 160

76 Comparison of different disturbance amplitudes with DREs spaced $4.5 \mathrm{~mm}$ apart

$77 \mathrm{~N}$-factor plot isolating the $4.5 \mathrm{~mm}$ and $2.25 \mathrm{~mm}$ wavelengths for the root pressure port row

$78 \mathrm{~N}$-factor plot isolating the $4.5 \mathrm{~mm}$ and $2.25 \mathrm{~mm}$ wavelengths for the tip pressure port row 
79 Demonstration of SWLFC with [12/2.25I1] (Root $R e_{k}=1.9$,

Tip $\left.R e_{k}=2.1, k / \delta_{\text {root }}=0.05, k / \delta_{\text {itp }}=0.05\right)$ control DREs

80 Demonstration of SWLFC with [25|2.25|1] (Root $R e_{k}=9.0$,

Tip $\left.R e_{k}=10.3, k / \delta_{\text {root }}=0.10, k / \delta_{\text {tip }}=0.10\right)$ control, variable-height

DREs . ....

81 Demonstration of SWLFC with [12/2.25I1] (Root $R e_{k}=1.9$,

Tip $\left.R e_{k}=2.1, k / \delta_{\text {root }}=0.05, k / \delta_{\text {tip }}=0.05\right)$ control, appliqué DREs

82 Demonstration of SWLFC with [12/2.25/1] (Root $\operatorname{Re}_{k}=1.9$,

Tip $\left.R e_{k}=2.1, k / \delta_{\text {root }}=0.05, k / \delta_{\text {tip }}=0.05\right)$ control, appliqué DREs

at the control neutral point

83 Surface scans over the appliqué DREs applied to the polished leading edge, painted leading edge near the root, and the painted leading edge near the tip

84 Schematic showing where the two different roughness scans were performed for Figure 83 .

85 Plot of transition Reynolds number for the polished leading edge and painted leading edge with and without [k/2.25/1] control, appliqué DREs

86 Bug strikes as a function of takeoff time. 188

87 Bug strikes as a function of ground temperature... 189

88 Bug strikes as a function of ground atmospheric pressure 189

89 Bug strikes over the course of a calendar year..... 190

90 Bug strikes as a function of relative humidity 190

91 Bug strikes as a function of wind speed. 191

92 Percentage loss of laminar flow due to a bug strike and assuming transition at $80 \% x / c$. 
FIGURE

Page

93 Percentage loss of laminar flow on the SWIFT model as a function of ground temperature

94 Percentage loss of laminar flow on the SWIFT model as a function of atmospheric pressure

95 Percentage loss of laminar flow on the SWIFT model as a function of relative humidity

195

96 Percentage loss of laminar flow on the SWIFT model as a function of wind speed 


\section{LIST OF TABLES}

TABLE Page

$1 \quad$ Surface roughness examples measured at the FRL .............................. 45 


\section{INTRODUCTION}

\subsection{Overview and Motivation}

This dissertation describes a series of flight tests conducted at the Texas A\&M Flight Research Laboratory (FRL) to study the effects of micron-sized Discrete Roughness Elements (DREs) on a 30-degree swept-wing. The swept-wing test article used here was designed to better understand crossflow-dominated transition applicable to SensorCraft technology (Martinez et al. 2008). Advances in lightweight composite construction, active flight controls, and aerodynamics are all needed to meet the lofty goals set forth for the notional SensorCraft vehicle. This research endeavor focuses on the aerodynamic aspects of SensorCraft and more specifically the design of a laminar flow swept-wing under the project title of Swept-Wing In-Flight Testing (SWIFT).

It is well understood that range and endurance are strongly dependent on the aircraft Lift over Drag ratio, $L / D$. Range and endurance scale proportionally to $L / D$. Improvements in lift can be made, but one of the most promising improvements in $L / D$ is achieved by reducing the drag. Laminar flow on the wings, empennage, and parts of the fuselage is the most promising technology for reducing the overall drag on a flight vehicle (Green 2008). Laminar flow has been a goal for aerodynamicists and aircraft designers for decades, but has only been incorporated into a few designs. Above and

This dissertation follows the style of Journal of Fluid Mechanics. 
beyond the increase in range and endurance, a laminar flow wing will produce less drag, decreasing the required engine thrust, resulting in less fuel burn and less engine noise. With the cyclical nature of high fuel prices, both SensorCraft and every aerodynamic vehicle can benefit from laminar flow.

The targeted Reynolds number for the SensorCraft UAV is 6.5 to 7.5 million. The leading edge sweep, $\Lambda_{L E}$, of the vehicle is 30 degrees. Beyond approximately 15 to 20 degrees of $\Lambda_{L E}$, a wing is subject to crossflow that cannot be stabilized by a favorable pressure gradient. Designers in the past have avoided high sweep angles for this very reason. The unswept wing, or 2-D wing, is well understood and laminarization can be achieved by tailoring the pressure distribution on the airfoil. Research in this area has led to the NACA series of Natural Laminar Flow (NLF) airfoils and several flight experiments and production aircraft have demonstrated this concept with good results.

This series of flight tests is a successor to approximately 15 years of research focusing on laminarizing swept-wings with the use of Discrete Roughness Elements (DREs). Promising results have been achieved in the experiments of Saric et al. (1998), where an innovative technique of applying passive roughness near the leading edge of a 45-degree swept-wing delayed transition beyond the naturally occurring location. These experiments were performed in a low-turbulence wind-tunnel and limited to Reynolds numbers up to 3.0 million for a $1.83 \mathrm{~m}$ chord model at $30 \mathrm{~m} / \mathrm{s}$. This technique was then applied to a 30-degree swept-wing operating at Mach 1.85 on an F-15 at NASA Dryden 
operating at a chord Reynolds number of 10 million (Saric et al. 2005). Almost full chord laminar flow was achieved on part of the model at Mach 1.85 and Mach 0.91, despite being faced with a spanwise flow variation across the model generated by the fuselage of the F-15. The current set of flight experiments attempts to repeat the success obtained in these past experiments, and attempts to get one step closer to developing DREs as a viable Laminar Flow Control (LFC) technique for future vehicles like SensorCraft.

\subsection{Crossflow Dominated Transition}

There are several instabilities that can lead to boundary-layer transition on a swept-wing. These include streamwise viscous instabilities such as Tollmien-Schlichting (T-S) waves, centrifugal instabilities (Görtler vortices), attachment-line contamination, and crossflow vortices. A recent review of the instabilities on a swept-wing can be found in Reed \& Saric (2008). The pressure distribution on the SWIFT model has been tailored to stabilize the T-S waves by incorporating an aft pressure minimum at $70 \% x / c$. Görtler vortices are eliminated on the model because concave curvature has been avoided near the leading edge. Attachment-line contamination has been avoided by designing the SWIFT model with a free end, not attached to any turbulent boundary layers. These design features isolate the remaining crossflow instability which is destabilized by the favorable pressure gradient used to stabilize the T-S waves. This leads to a category of LFC titled Swept-Wing Laminar Flow Control (SWLFC). SWLFC takes into account all the features of NLF with a slight favorable pressure gradient, but incorporates spanwise- 
periodic roughness at the leading edge for added control of the unstable crossflow vortices. It is this class of LFC that was studied in the SWIFT flight test program described here.

To understand the crossflow instability, and the control technique of applying DREs at the leading edge, one needs to first understand the physics within the boundary layer of a swept-wing. On a swept-wing the inviscid streamlines approaching the leading edge of the wing first deflect away from the fuselage, for a traditional swept-back wing, due to the fact that the flow must be parallel to the leading edge at the attachment line. Aft of this point the favorable pressure gradient starts to force the streamlines back towards the fuselage, until they deflect again away from the fuselage aft of the pressure minimum to recover the pressure at the trailing edge. Figure 1 shows an idealized inviscid streamline over a swept-wing. Because the static pressure is constant through the boundary layer, $d p / d y=0$, the centripetal force generated by the curved streamline is opposed by the pressure gradient at the edge of the boundary layer, but at the surface only the pressure gradient is present. This generates the crossflow boundary-layer velocity, perpendicular to the inviscid streamlines and zero at the surface and zero at the boundary-layer edge. Figure 2 shows a schematic of the crossflow boundary layer. 


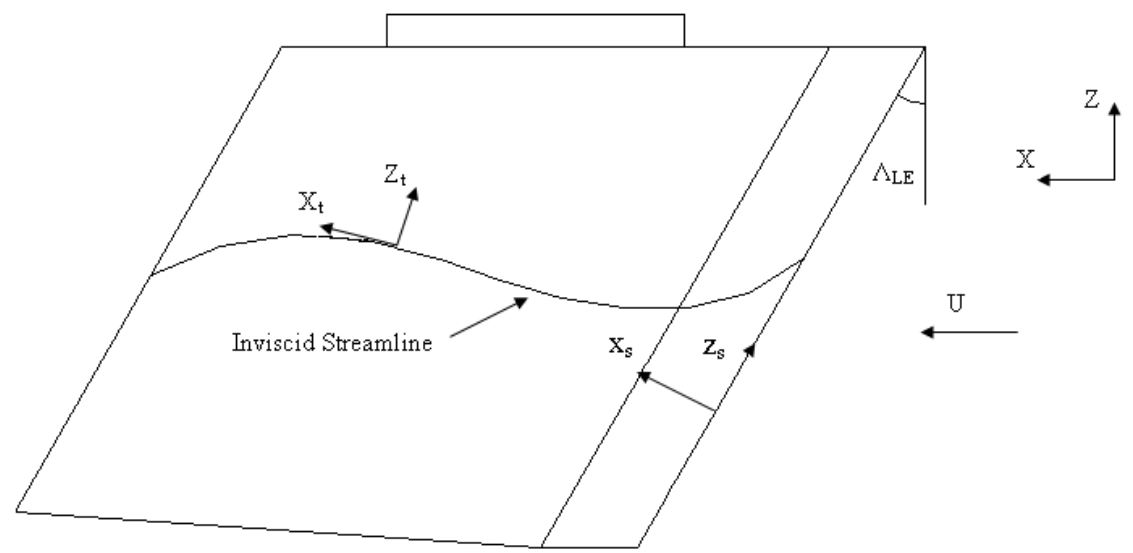

Figure 1: Inviscid streamline over a swept-wing and standard coordinates used to describe swept-wings.

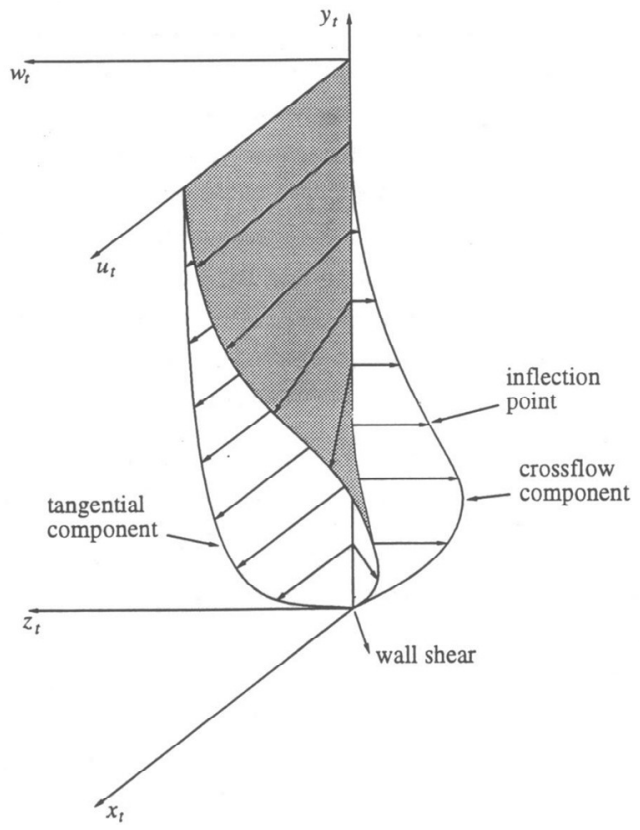

Figure 2: Swept-wing boundary layer profile showing the tangential, crossflow, and total velocity profiles. Figure courtesy of Saric et al. (2003). 
The crossflow component has an inflection point which has been proven to be unstable in the inviscid limit. On the swept-wing the crossflow instability manifests itself as growth in the form of co-rotating vortices, where low momentum fluid at the surface is drawn up higher in the boundary layer, while higher momentum fluid is forced lower in the boundary layer towards the surface. This produces co-rotating crossflow vortices forming around the crossflow inflection point that ultimately leads to and feeds into the high-frequency secondary instability resulting in eventual breakdown to turbulence. Comprehensive reviews for both two- and three-dimensional flows are given by Arnal (1994), Mack (1984), Reed \& Saric (1989), Reshotko (1994), and Saric et al. (2003). Figure 3 shows experimental contour plots showing the streamwise growth of the crossflow vortices taken from boundary layers scans in the experiments of Reibert et al. (1996). For this case DREs were applied to generate uniform crossflow vortices spaced $12 \mathrm{~mm}$ apart. More detail will be provided in Section 6 on the use of DREs. 

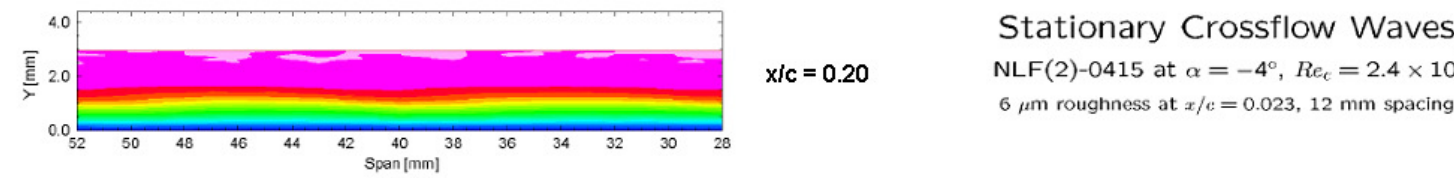

NLF(2)-0415 at $\alpha=-4^{\circ}, R e_{c}=2.4 \times 10^{6}$

$6 \mu \mathrm{m}$ roughness at $x / c=0.023,12 \mathrm{~mm}$ spacing

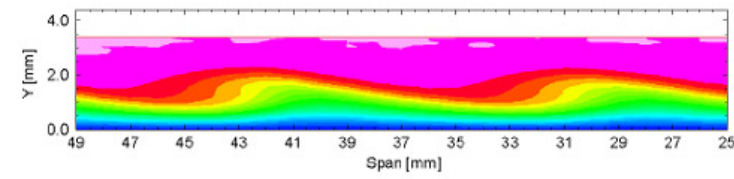

$x i c=0.30$

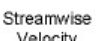
Velocity
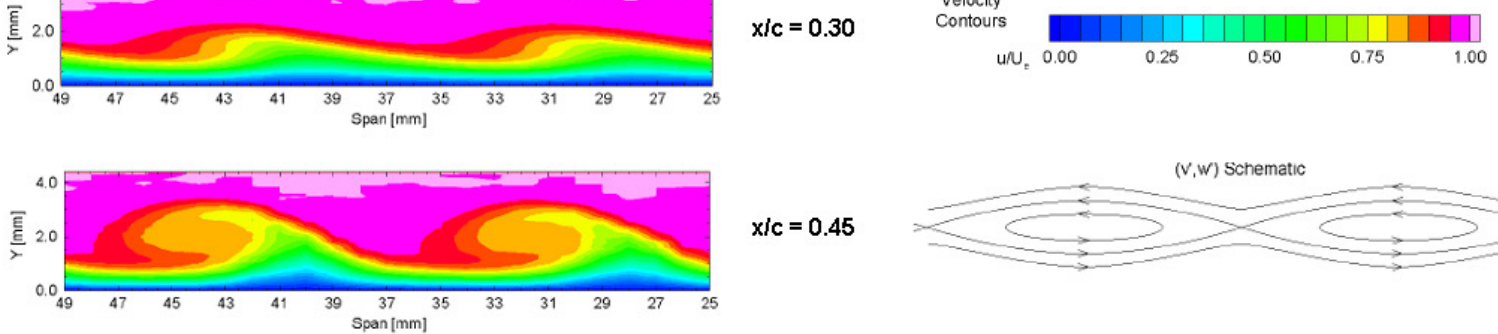

$x i c=0.45$

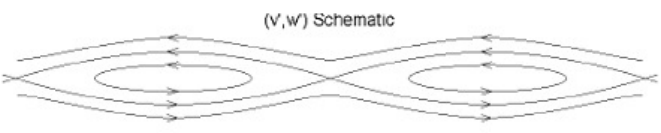

Figure 3: Development of stationary crossflow waves in the chordwise direction. Figure adapted from Reibert et al. (1996).

Linear Stability Theory (LST) and solution of the Orr-Sommerfeld equation show that a disturbance within the boundary layer can be characterized with a streamwise wavenumber $\alpha_{t}$, a spanwise wavenumber $\beta_{t}$, and an unsteady frequency $\omega$, in the form of Equation 1. Subscript $t$ has been added to distinguish the wavenumbers from the aircraft angle of attack, $\alpha$, and the aircraft sideslip angle, $\beta$ to be introduced in Section 2. The imaginary part of $\alpha_{t}$ is the growth rate in the streamwise direction. The real part of $\beta_{t}$ describes the spacing of the crossflow vortices. A swept-wing will experience both stationary $(\omega=0)$ and traveling $(\omega \neq 0)$ crossflow waves. Despite demonstrating higher growth rates, the traveling crossflow vortices typically do not lead to transition on the swept-wing in low-disturbance environments since it has been shown that the stationary crossflow waves dominate the transition process (Saric et al. 2003). On the other hand, traveling crossflow waves dominate in high-disturbance environments (Bippes 1997, Deyhle \& Bippes 1996). Since flight is generally 
characterized with low freestream turbulence, investigation of the stationary crossflow vortices is considered to be more important between the two problems. As a result the majority of this research endeavor focuses on, first understanding the destabilizing stationary crossflow vortices, and then controlling them by introducing discrete roughness at the leading edge to prevent the growth of the most unstable wavelength.

$$
\phi^{\prime}(x, y, z, t)=\phi(y) e^{i\left(\alpha_{t} x+\beta_{t} z-\omega t\right)}+c . c .
$$

\subsection{Experimental Objectives}

When studying boundary-layer transition on the swept-wing, the breakdown to turbulence can be divided into three categories. The first stage is receptivity where longwavelength disturbances in the environment or surface imperfections produce shortwavelength disturbances in the boundary layer (Saric et al. 2002). Once the initial disturbances are introduced through the receptivity mechanism, exponential growth occurs in the form of crossflow vortices. LST predicts the initial growth rate in this region well, but does not account for the amplitude saturation measured by Reibert et al. (1996) and calculated by Haynes \& Reed (2000). Lastly, the secondary, high frequency, instability dominates and transition to turbulence occurs over a very short distance (White \& Saric 2005). LFC can be applied at any one of the three stages. However, the receptivity region holds the most promise for LFC because linear growth and the secondary instability can all be controlled if the receptivity problem is understood and receptivity mechanisms are reduced. Most attention is spent investigating the linear 
growth region, whereas the receptivity region is the least understood and few experiments deal with this subject (Choudhari \& Streett 1990). This dissertation describes a series of flight experiments focusing on the receptivity problem for the swept-wing with an ultimate goal to provide SWLFC on a 30-degree swept-wing test article with the use of DREs.

Specifically the objectives for SWIFT were to design a 30-degree swept-wing to be operated under SensorCraft flight conditions. First, the crossflow instability is investigated by promoting early transition with the use of critically-spaced DREs. A new technique for measuring disturbance amplitudes with the use of calibrated surfacemounted hotfilms was then developed to make disturbance amplitude measurements as a function of modulating DRE heights. This measurement has never been attempted before, and is one of the last missing links for performing SWLFC with DREs. Lastly, the control problem of delaying transition was performed with subcritically-spaced DREs. 


\section{EXPERIMENTAL SETUP}

\subsection{Experimental Requirements}

Several options were available for studying the swept-wing test article needed for the SWIFT program. Both wind-tunnel tests and flight tests were considered. The most important requirement for conducting a stability experiment is to choose an environment that closely represents the conditions experienced by the final flight vehicle. There is an endless supply of transition experiments performed in noisy wind tunnels where the transition results are extrapolated to flight by performing empirical corrections such as the popular $e^{N}$ method, where $N$ is adjusted for noisy wind tunnels, low turbulence wind tunnels, or flight (Smith 1956, Smith \& Gamberoni 1956, Van Ingen 1956). These methods usually fail to predict transition on the flight vehicle because the fundamental instabilities leading to transition are not correctly matched in a noisy tunnel and the nature of the instability experienced in flight might be completely different. For example, the crossflow dominated swept-wing may experience traveling crossflow waves in a noisy wind tunnel but in flight will be dominated by stationary crossflow waves. In this case the defining parameter is the freestream turbulence. Flight is generally characterized as a low-disturbance environment with low freestream turbulence. Consequently the SWIFT experiments would need to be conducted in a lowturbulence wind tunnel, or in flight, to match the freestream turbulence levels for SensorCraft. 
To achieve the targeted Reynolds number, $R e_{c}$, of 6.5 to 7.5 million requires a large wind tunnel capable of achieving high speeds and capable of supporting a large model. Unfortunately all wind tunnels suffer from an increase in freestream turbulence as the velocity in the test section increases. Eventually a speed is reached where the freestream turbulence exceeds the values needed to be considered a low disturbance environment necessary for these experiments. Very few wind tunnels can claim low disturbance conditions at the speeds required for SensorCraft, thus a flight experiment was selected as the more appropriate option.

An aircraft was needed that was relatively cheap to operate, capable of carrying a large swept-wing test article, and fast enough to meet the $R e_{c}$ requirement from 6.5 to 7.5 million. A secondary requirement was to match the SensorCraft Mach number of 0.6. Mach number was considered to be of secondary importance because Mach number corrections can always be applied after the data are collected. If one were conducting a supersonic experiment and studying shock wave/body interaction, then Mach number would be the most important parameter. However, when one looks at boundary-layer transition, the $R e_{c}$ is the defining variable. Last, but not least, the aircraft needed to have areas of "clean" airflow where a test article could be mounted away from propeller or jet wash, engine exhaust, and static pressure fluctuations from the rest of the aircraft.

There were three options considered for the design of the swept-wing test article. One could replace the existing wing of the test aircraft and build a new wing, such as in 
the X-21 program where a Douglas B-66 aircraft was modified with a new laminar flow swept wing (Fowell \& Antonatos 1965). A second option is to install a wing glove over the existing wing as in the F-16XL experiments (Woan et al. 1991), B-757 flight tests (Collier 1993), and the NASA Jetstar flight tests (Fischer et al. 1983, Maddalon \& Braslow 1990), to name a few. In these cases the new airfoil shape was built up over the existing wing. Lastly, one could mount an entire wing section somewhere on the aircraft as in the supersonic experiments on the F-15 at NASA Dryden (Banks et al. 2000). This third option of mounting an entire wing section somewhere on the aircraft was chosen for the SWIFT program because it resembled a wind tunnel experiment, which was familiar for most members of the FRL. Moreover, there are safety concerns with replacing and or modifying an existing wing on an aircraft. It was decided early on in the program that the objectives would be met with as little modification to the test aircraft as possible. A wing glove was also rejected because the glove would affect the flight characteristics of the aircraft and there is always the added complexity of fairing the design into the existing airfoil shape at the trailing edge of the glove. An external store allowed complete control over the size, shape, and surface finish of the entire model from leading edge to trailing edge. As a result an aircraft was selected capable of carrying large stores, where the test article would be an external attachment somewhere on the aircraft. Furthermore, the mounting location would be ideally located near the center of gravity of the aircraft to reduce any moments generated by the swept-wing test article. 


\subsection{Test Aircraft}

A 1968 Cessna O-2A Skymaster was purchased specifically for this series of experiments and was dedicated to the program for the majority of the time period. The $\mathrm{O}-2 \mathrm{~A}$ is a high-wing, twin-engine aircraft featuring the distinctive twin tail and centerline thrust configuration. The centerline thrust was designed to provide added safety in the event of an engine failure, eliminating the yawing moment produced by an engine out on a conventional twin. The O-2A is a military modification of the Cessna 337 and includes four wing-mounted pylons, reinforced spars for cyclic loading, observation windows on both the pilot and co-pilot sides, a third-row radio rack in the cabin, and jettisonable cabin doors and pilot-side window in the case of an emergency evacuation. Figure 4 shows a picture of the unmodified aircraft in addition to a dimensioned three-view drawing. The Cessna O-2B had a different mission and most notably did not have the four wing-mounted pylons. As a result the O-2A designation will be used throughout to distinguish it from the O-2B aircraft. 

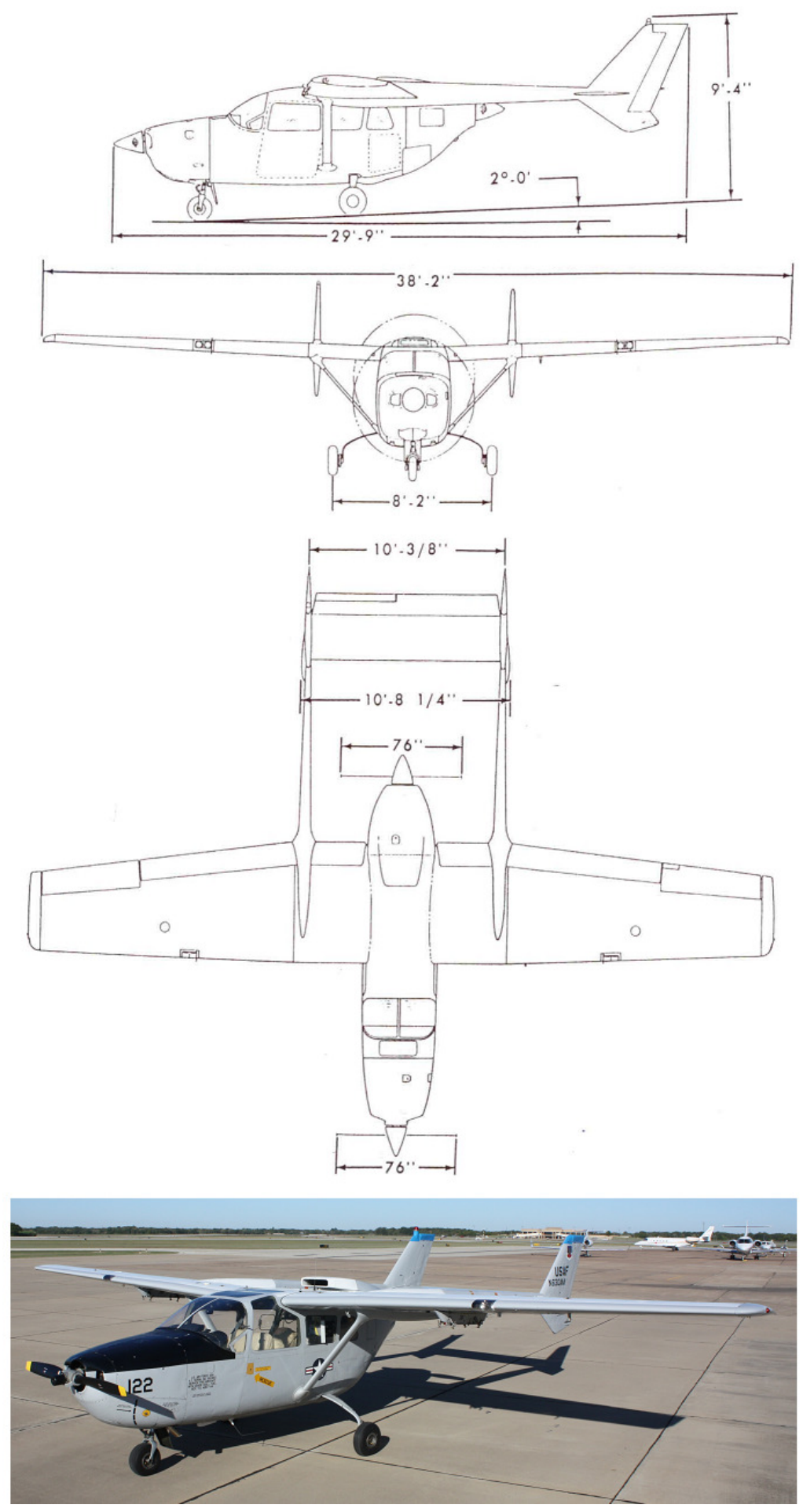

Figure 4: Unmodified test aircraft with dimensioned three-view drawing. 
Of all the features incorporated into the O-2A design, the wing-mounted pylons were specifically targeted for mounting a large swept-wing test article. A model could be designed and mounted to one of the pylons which already had a rich military history of carrying large stores. Furthermore, the Cessna O-2A's military history provided copious amounts of documents which assisted in the risk mitigation part of the SWIFT experiments. Documents ranging from aircraft performance (Rydman \& Palmer 1969), static load tests on the pylons (Van Dyke 1970), pylon fatigue tests (Davis 1968), ground vibration tests, and flutter analysis (Pate 1967) were all available for the O-2A aircraft. The aircraft's historical reputation was important in providing a foundation for flutter clearance and identifying weak points in the pylons which helped greatly when performing the safety analysis.

One disadvantage of using the Cessna O-2A for this experiment is its top-speed limitation. During level flight the O-2A cruises around 130 knots true airspeed, KTAS. To achieve the targeted $\operatorname{Re}_{c}$ for SensorCraft the model would have to be either very large, or the speed of the aircraft would have to be increased. Weight and safety concerns prevented the model from being excessively large, and as a result a compromise was made by increasing both the size of the model and the top speed of the aircraft. In order to increase the speed of the aircraft, the flight profile would have to include a dive. This was not limiting because a temperature differential was needed for identifying boundary-layer transition, which will be described in Section 5. Internal cooling and heating of the model was considered, but in the end it was decided to design 
a smaller model for weight and safety concerns, use the temperature lapse rate in the atmosphere for visualizing boundary layer transition, and increase the top speed of the aircraft by initiating a dive for all test points. This technique required no additional weight or complexity for cooling or heating the model, but suffers from a reduced run time during the dive in addition to a lengthy cold soak time at altitude. All data would have to be acquired during a limited time interval in the dive; typically only three minutes on test conditions were available before the test pilot would level off. Since the Reynolds number is the most important parameter for stability and transition studies, the dives would be accomplished under conditions of constant $R e_{c}$. Furthermore, all dives would be made at the lowest possible safe altitude to benefit from the decrease in kinematic viscosity closest to sea level in order to achieve the targeted $\operatorname{Re}_{c}$ of 7.5 million.

The aircraft was registered in the Restricted category in order to allow modifications to the type-certificated airframe. As a result, the FAA issued operating limitations were specific to this aircraft. Flight operations with the model were to be conducted outside congested areas with the exception of takeoff and landing. Flight was limited to day VFR flight only, and only necessary crewmembers could fly when the model was attached. 


\subsection{Swept-Wing In-Flight Testing (SWIFT) Model}

The test article designed for these experiments is an aluminum model with each component being milled out of a billet of 6061-T6 aluminum. Aluminum was chosen over a composite model because FRL members had more experience with machining practices, surface finish, and waviness capabilities of aluminum over a composite model. This resulted in a weight penalty over lightweight carbon fiber, but was within the operating limitations of the Cessna O-2A aircraft.

Starting with the leading edge, the SWIFT model has a leading edge sweep of $\Lambda_{L E}=30$ degrees, per the requirements for SensorCraft. A chord of 54 inches $(1.37 \mathrm{~m})$ was chosen to provide the targeted $R e_{c}$ between 6.5 and 7.5 million within the performance capabilities of the O-2A during a dive. The span of the SWIFT model was 42 inches at the leading edge, but a 4-degree cut starting aft of the leading edge was incorporated for added ground clearance during rotation at takeoff and landing. The model has two interchangeable leading edges depending on the given flight test. Leading edge \#1 has static pressure ports. This leading edge was used for all initial flying-quality flights and Pressure Coefficient $(C p)$ measurements. Leading edge \#2 had no pressure ports and demonstrated the DRE technology and hotfilm receptivity measurements. Both leading edge attachments have the same dimensions and were mounted to the rest of the airfoil at the $15 \%$ chord position. The rest of the airfoil, from the $15 \%$ chord position to the trailing edge, was a powder-coated surface used to provide a non-reflective surface for the infrared camera, in addition to providing a thin insulating barrier to reduce heat 
conduction across the transition front. The powder-coat finish was black to provide the highest emissivity possible for the infrared camera. The interior cavity of the model was milled out to provide the lightest possible structure, but still strong enough to withstand the aerodynamic loads with a necessary factor of safety. All interior stiffeners were milled out of the design from a billet of aluminum resulting in a model composed of three main parts: the test-side surface, the non test-side surface, and the leading edge. The three main parts were assembled with all bolts on the non test side of the model, leaving the test side of the model disturbance free.

Preliminary safety analysis determined that the SWIFT model required a strut to support the model and alleviate some of the loads experienced in the pylon. The strut selected was a streamlined aluminum extrusion normally intended as a sailplane strut. It attached to a stainless-steel plate at the bottom of the model and extended up to one of the tie-down hardpoints in the wing. The strut was on the backside of the model and did not obstruct the view of the test side during the experiment. Furthermore, a study was conducted to verify that the strut did not influence the pressure distribution on the testside of the model. Rhodes et al. (2008) showed that the strut could be rotated as much as \pm 20 degrees angle of attack and still not alter the pressure distribution on the test side of the model. Figure 5 shows the SWIFT model assembly resulting in a final weight of 242 lbs. Depicted in Figure 5 is the mounting structure, top plate, test side, non test side, bottom plate, leading edge, strut, and strut attachment plate. All components were aluminum with the exception of the stainless-steel plates at the top and bottom of 
the model to hold the two halves together. The hollowed out internal structure of the non test side of the model is also visible in Figure 5.

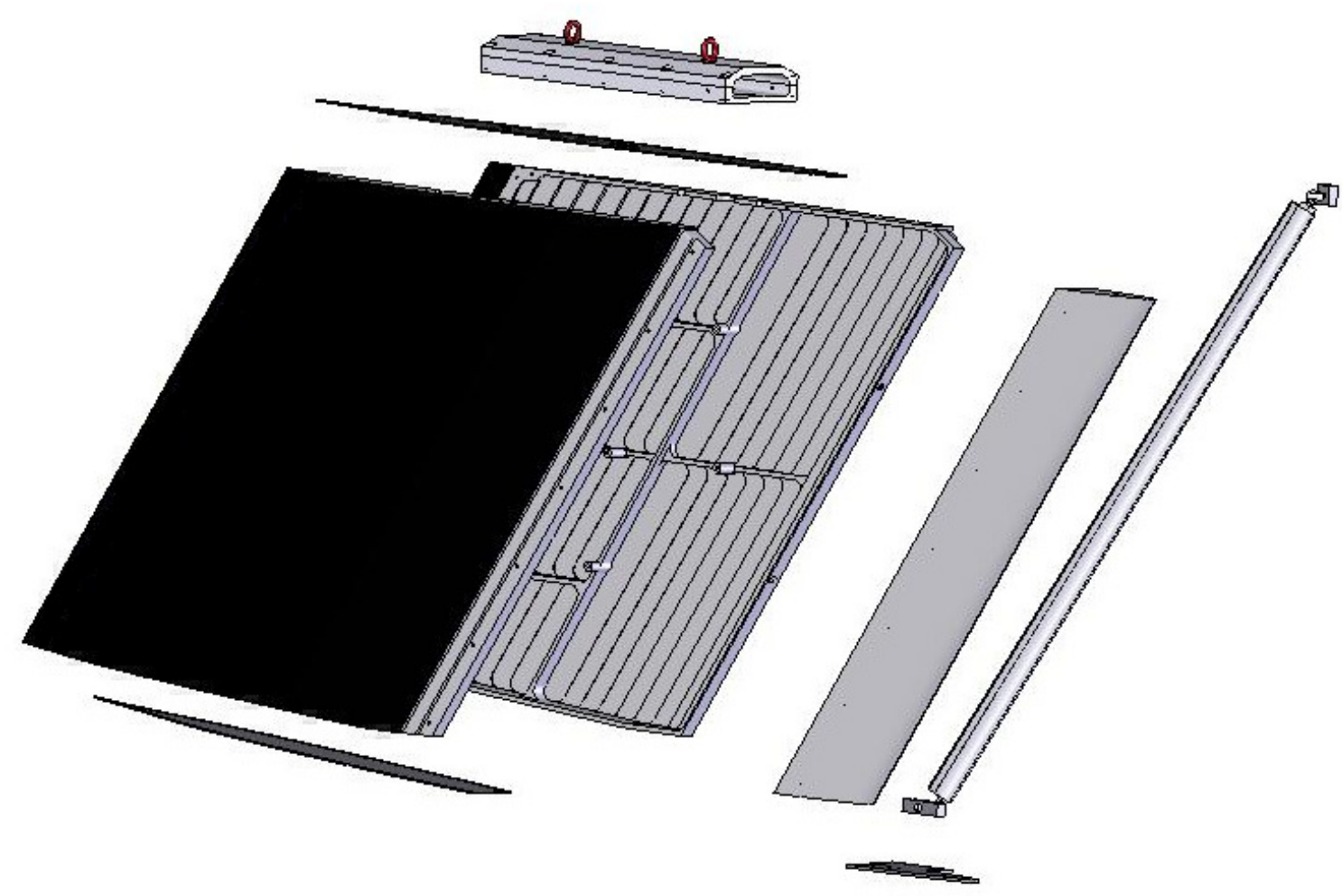

Figure 5: Breakaway view of the major SWIFT components showing the black powdercoat test side of the model.

The airfoil coordinates of SWIFT were designed, not as a final wing design for SensorCraft, but rather an experimental test article intended to study the effectiveness of the DRE technology. It was desired that the SWIFT model would generate zero lift at zero angle of attack. This helped reduce some of the aerodynamic loads on the model and aircraft during non-testing conditions. The leading edge was symmetric up to $15 \%$ 
$x / c$. Beyond $15 \% x / c$ the airfoil on the non test side was designed to cancel out any lift generated on the suction side of the model, with the test side considered to be the suction side of the model. The shape of the airfoil coordinates also needed to generate enough crossflow to demonstrate crossflow-dominated transition at 7.5 million $\operatorname{Re}_{c}$ and 30 degrees of $\Lambda_{L E}$. The model was designed to transition to turbulence around $50 \% x / c$ at 7.5 million $R e_{c}$ based on an $N$-factor of 9. Lastly the airfoil needed to be subcritical to T$\mathrm{S}$ waves in order to isolate the crossflow instability.

The mounting structure used to mate the SWIFT model with one of the O-2A's wing-mounted pylons is also depicted in Figure 5. The mounting structure has two eyebolts spaced 14 inches apart to line up with the standardized military MA-4 bomb rack. In addition to supporting the model, the mounting structure also has the ability to change the incidence of the model on the ground. This feature was used later in the program to rotate the leading edge of the model away from the fuselage in order to promote negative angles of attack. This feature in the mount design allowed some of the large negative angles of attack without forcing the aircraft to fly at extreme sideslip angles. Some of the settings used with the mounting structure are described in Section 4.

\subsection{Aircraft Configuration and Coordinate System}

During all phases of the experiment the aircraft was configured with three crewmembers. A test pilot occupied the front left seat. A safety observer occupied the 
front right seat with the primary objective of operating the radio and watching for traffic, especially during the dive portion of the experiment when the test pilot was focused on maintaining test conditions. The third crewmember was the flight test engineer (FTE) who occupied the rear right seat behind the safety observer. The third row in the O-2A was equipped with a radio rack and most of the instrumentation in the cabin was mounted there. An infrared camera occupied the rear-left-seat location and was pointed out towards the SWIFT model for boundary-layer transition detection. With the SWIFT model attached to the port wing, the hotwire sting was placed at the starboard outboard pylon position for lateral balance. More information on the hotwire sting is provided in Section 3 regarding freestream disturbance measurements. Figure 6 is a schematic of the finalized configuration of the SWIFT model and Cessna O-2A aircraft used for these experiments. Figure 7 complements Figure 6 with photographs. In both Figures 6 and 7 a five-hole probe is shown attached to the SWIFT model. More information on this probe is presented later in this section. 

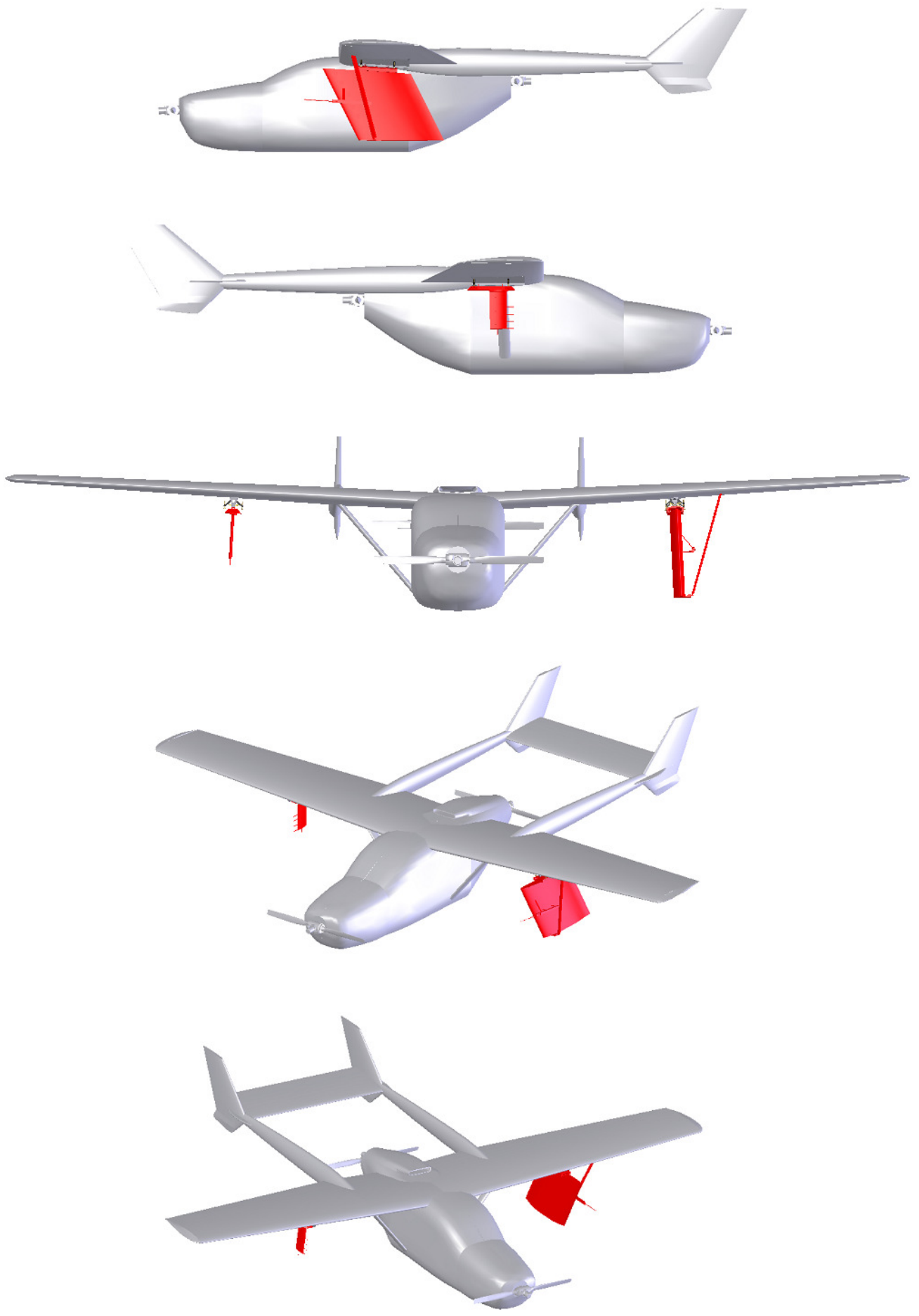

Figure 6: Schematic of SWIFT model with five-hole probe and the hotwire sting. 

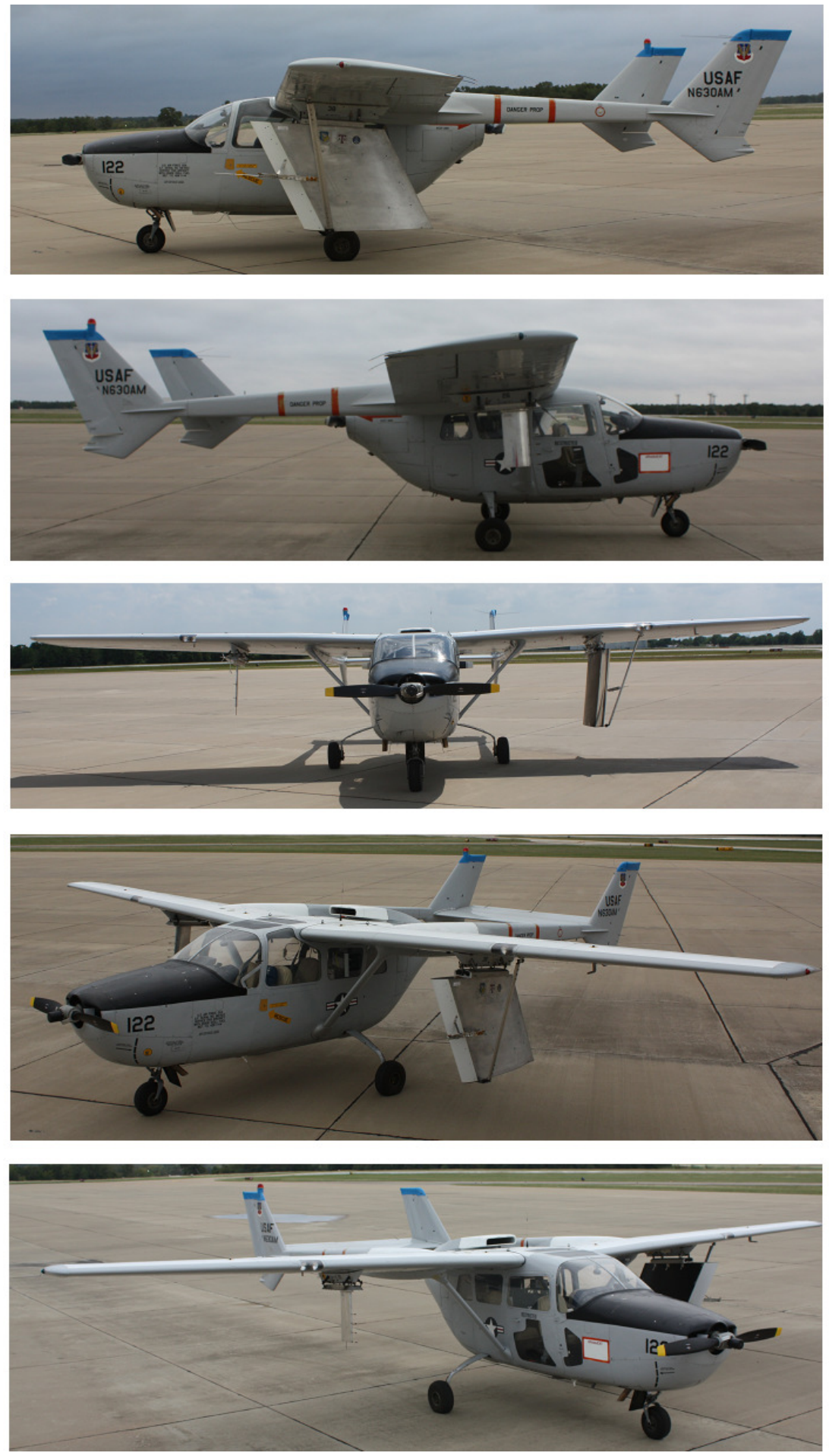

Figure 7: Pictures of SWIFT model with five-hole probe and the hotwire sting. 
A new coordinate system was developed for studying this unconventional, vertically-mounted airfoil. The aircraft angle of attack, $\alpha$, corresponds to a change in sweep on the SWIFT model. An increase in $\alpha$ decreases $\Lambda_{L E}$. The aircraft sideslip angle, $\beta$, corresponds to the angle of attack on the SWIFT model. However, to keep the convention for positive $\beta$, the SWIFT angle of attack is the opposite of $\beta$. Negative angles of attack on SWIFT correspond to positive $\beta$ values. Because of the sometimes confusing nature of the symbols, $\beta$ always refers to aircraft sideslip, and where necessary the SWIFT angle of attack is denoted as AoA. $\alpha$ is always referenced as aircraft angle of attack, and changes in sweep on SWIFT is denoted with $\Lambda_{L E \text {. Equations }}$ 2 and 3 show the relationship between the four variables of interest.

$$
\Lambda_{L E}=30^{\circ}-\alpha
$$

$$
A o A=-\beta
$$

Past wind-tunnel experiments within this research group have developed standardized figures for the coordinate systems used to describe swept wings. However, these experiments were always conducted with flow going from the left to the right. To reduce any confusion, Figure 1 is referenced again as the appropriate coordinate system for this experiment. The freestream velocity components are $U, V$, and $W$. When following one of the inviscid streamlines on the swept-wing another coordinate system is 
convenient to use. The velocities $u_{t}, v_{t}$, and $w_{t}$, are the velocity components tangential, wall normal, and orthogonal to the streamline, respectively. The third coordinate system is referenced normal to the leading edge and is also denoted with lowercase variables as $u_{s}, v_{s}$, and $w_{s}$. Moreover, Figure 8 shows the coordinate system defining the freestream velocities and angles $\alpha$ and $\beta$ used for the SWIFT experiments.

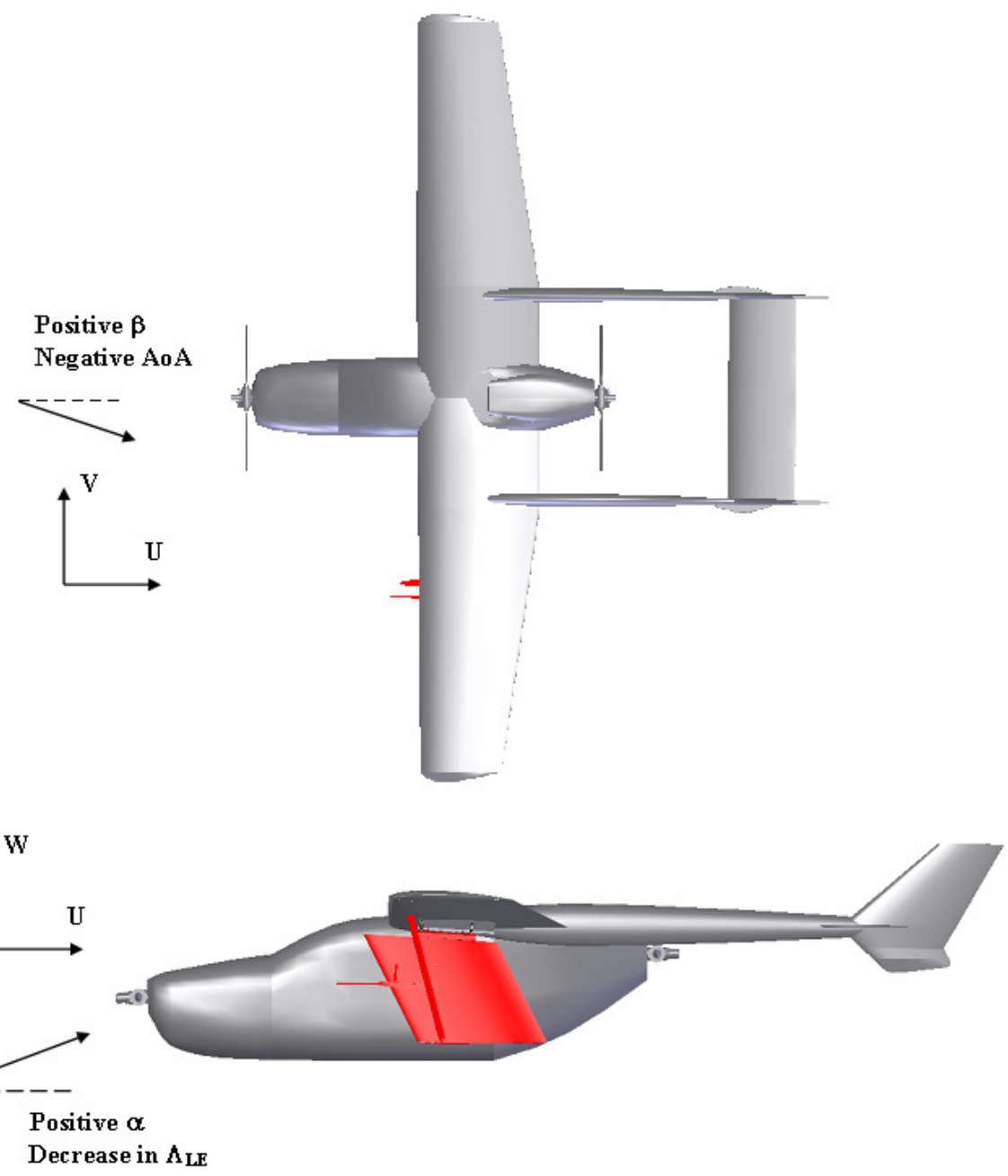

Figure 8: Freestream coordinate system for velocities and angles $\alpha$ and $\beta$. 


\subsection{Safety Analysis}

Prior to the first flight with the SWIFT model an extensive safety analysis was performed on the model and critical components of the aircraft. The FRL team performed the necessary load estimations, stress analysis, flutter analysis, and low-speed directional control analysis before the first flight. Furthermore, three static load tests were performed where flight hardware was loaded to 1.5 times the highest expected load. A thorough review of the analysis can be found in McKnight (2006) and Saric et al. (2006). Only the highlights of the analysis are mentioned here, as these two documents explain the analysis in more detail.

The SWIFT model and aircraft are designed to withstand a 7-degree AoA (30degree bank) on the SWIFT model at 165 knots indicated airspeed, KIAS, combined with a 2-g pull up maneuver. This maneuver was envisioned as a possible collision avoidance maneuver with another aircraft during the high-speed dive. Under these conditions the model generates approximately $500 \mathrm{lbs}$ of lift. With a model weight of $242 \mathrm{lbs}$, the $2-\mathrm{g}$ pull-up maneuver results in a $484 \mathrm{lb}$ vertical load down. The drag load and pitching moments were considered negligible for the analysis and were ignored in both the stress analysis and static load tests.

McKnight (2006) built a finite-element model of the structure and analyzed every component of SWIFT, the O-2A pylon, and the hardpoints attached to the O-2A leadingand trailing-edge spars. The results of the analysis were twofold. First, the analysis 
identified weak components in the assembly requiring reinforcements, and second, the analysis determined the factors of safety of each component. The stress analysis exposed the pylon as the weakest part of the entire assembly. The aircraft routinely carried large external stores in the form of SUU-14A bomblet dispensers, LAU-59/A rockets, and SUU-11A/A miniguns. However, when one examines the geometry, and more importantly the aerodynamic loads of these military stores, the pylon was designed to withstand mostly drag and thrust loads generated from the reaction force of a fired rocket or minigun. The pylon was also designed to support inertial loads up to the rated amount of $3.8 \mathrm{~g}$ vertically down. The worst case scenario already tested with the original weaponry was a $1900 \mathrm{lb}$ aft load created by the firing of a LAU-59/A rocket. The highest inertial load was from the $325 \mathrm{lb} \mathrm{SUU}-11 \mathrm{~A} / \mathrm{A}$ minigun at $3.8 \mathrm{~g}$ down resulting in 1235 lbs pulling down on the bomb rack and pylon (Davis 1968). However, these extreme loads are very different from the lifting loads generated by the geometrically larger SWIFT model. This resulted in the addition of the strut on the back side of the model and the redesign of some critical components in the pylon. In the end, the analysis determined that every component was designed with a factor of safety greater than 2 to yield. After all analyses were completed, three static load tests were conducted with loads applied up to 1.5 times the worst load case on flight hardware.

Despite being lighter than most of the weapons already flown, the SWIFT model had the highest pitch inertia about the aeroelastic axis of the O-2A wing because its center of gravity, CG, was much lower than any store ever flown before. For example, the minigun was mounted 8 inches below the pylon, whereas the SWIFT model's CG is 
24 inches below the pylon. However, the ground vibration tests performed by Cessna on all wing stores and even ballasted $350 \mathrm{lb}$ SUU-14A bomblet dispensers showed that the flutter mechanism for this aircraft was independent of wing stores (Pate 1967). Because the rotational moment of inertia of the SWIFT model was close to the pitch inertia of the $350 \mathrm{lb}$ bomblet dispenser, and less than the combined effect of two $250 \mathrm{lb}$ bomblet dispensers, the model was cleared for flutter through similarity.

Furthermore, a conservative flying-qualities and flutter-clearance campaign was initiated after all the safety analyses were completed and is best described in Martin et al. (2008). Pilots Roy Martin and Dr. Donald T. Ward, two pilots with over 70 years and 16,000 hours of combined military flight-test experience, cleared the envelope reducing any concern regarding low-speed directional control and high-speed flutter. After 19 flights and 23.5 flight hours of preliminary flying quality flights with and without the SWIFT model, the two test pilots made recommendations for limiting the flight envelope for the experiments.

\subsection{Flight Restrictions}

The 54 inch $(1.37 \mathrm{~m})$ chord model provided the necessary size to achieve the targeted $R e_{c}$ when the aircraft initiates a dive at 165 KIAS. The aircraft is rated for higher speeds, but only in smooth conditions. The Never Exceed Speed, $V_{N E}$, of the aircraft is 192 KIAS. It was decided to limit the dive speed to the Top Speed in Turbulent Atmospheric Conditions, $V_{N O}$, in case any turbulence was encountered during the dive. 
The $V_{N O}$ speed is 165 KIAS and was used as the targeted speed for early tests. However, during the flutter clearance flights the test pilots initiated a dive at $175 \mathrm{KIAS}$, even though the targeted flutter clearance speed was 5 knots less at 170 KIAS. No signs of flutter were observed at this speed. As a result, the aircraft was cleared for flutter at 170 $K I A S$ and this speed was used as the targeted condition for the dive, but only in perfectly smooth conditions. Whenever moderate atmospheric turbulence was encountered the test pilots always reduced speed down to the 165 KIAS $V_{N O}$ condition.

With the asymmetric wing loading, the Pilot's Operating Handbook (POH) provided limitations for crosswind takeoffs. With a $210 \mathrm{lb}$ differential weight $(242 \mathrm{lb}$ SWIFT model on the port wing and the $32 \mathrm{lb}$ hotwire sting on the starboard wing), the crosswind limitation was 4 knots. Despite always taking off with more fuel in the starboard tank, the $\mathrm{POH}$ did not allow for differential fuel loading as a means for increasing the crosswind limitation. Only external wing stores could be added for decreasing the asymmetric loading limitation. Four knots was a strict crosswind limitation, but rarely resulted in a canceled flight with the three intersecting runways available at Easterwood Airport.

The POH allowed for CG limits between 137 and 143 inches aft of the datum. Early flying-quality flights with the test pilots demonstrated that the O-2A aircraft had little directional stability at low speeds and aft CG locations. The two test pilots suggested the aft CG limit be placed at 140.5 inches initially and then later cleared for 
141 inches to accommodate additional instrumentation needed for later flights. This limitation affected the mounting locations for some of the heavier instruments. For example, the 50-lb anemometer used for freestream turbulence and hotfilm receptivity measurements could never be mounted on the aft instrument rack. A separate instrument rack was fabricated for mounting it behind the pilot's seat at a forward CG location.

\subsection{Flight Instrumentation}

During all flights, the FTE operated all of the instrumentation located on the third row instrumentation rack. The FTE held a Dell Precision M65 laptop running Windows XP. A Magma 4-slot PCI-PCMCIA bridge provided the necessary interface between the PCI DAQboards used for data acquisition and the laptop PCMCIA card. Two National Instruments analog to digital DAQboards were used. The first board was a 12-bit analog input board, PCI-6071E, which was used for all data acquisition. The PCI-6071E has 32 differential analog input channels and could sample up to $1.25 \mathrm{MHz}$ for one channel. This board also has two analog outputs in which one channel was used for the electronic pressure regulator for controlling the variable-height DREs explained in Section 6. A second 13-bit DAQboard was used for 2 channels of analog output for displaying the $R e_{c}$ and $\beta$ for the pilot on two digital displays. This board was a PCI-6723 DAQboard and has a total of 32 channels of analog output capable of $800 \mathrm{kHz}$ output rate for one channel. 
Power for all instrumentation requiring $115 \mathrm{VAC}, 60 \mathrm{~Hz}$ power came from an Exeltech 600-Watt power inverter. The inverter took supply power from the 24-volt aircraft battery which was drawing power from the aircraft's electrical bus. The inverter generated a sine wave with a maximum distortion of $2 \%$. A 40-amp circuit breaker provided circuit protection for all AC and DC power used for the experiments. A "kill switch" was wired up to the pilot's instrument panel so that at any time the test pilot had control over all the AC powered instruments running in the back. This "kill switch" was used as an emergency switch for shutting down all instrumentation in an emergency, in addition to standard on/off procedures for the inverter. The aircraft has two 60-amp alternators, one on each engine. This available electrical power was more than enough to run the instrumentation for SWIFT which usually ran less than 30 amps of current during the experiment. The aircraft instruments generally used less than 10 amps of current.

The third PCI slot in the bridge was used for the FLIR infrared camera PCI board. The IR camera was used as the primary transition detection tool and is described in Section 5. The IR camera was attached to an articulating camera tripod mount which simply clamped onto the instrument rack. A safety strap was constructed and hooked to the roof of the cabin to help alleviate some the weight carried by the camera mount. The IR camera pointed out an open window to the left side of the FTE. The 8-9 micron spectral range of the camera could not transmit through the Plexiglas of the cabin so one of the rear windows was removed to avoid transmission losses. Furthermore, an air 
deflector was constructed out of sheet metal to deflect most of the air from entering the cabin. A 60-degree lens could easily capture the entire model. A 20-degree lens was available, but was only used on a few flights, as its field of view was small.

An Aeroprobe, conical-tip, five-hole probe was used for all freestream measurements of total pressure, static pressure, $\alpha$, and $\beta$. The five-hole probe was 24 inches long and mounted to the non-testside of the SWIFT model. The six pressure ports on the five-hole probe led to four Honeywell Sensotech FP2000 pressure transducers inside the internal cavity of the model. Three of the pressure transducers were differential units with a full scale of \pm 2 psid and an accuracy of 0.002 psid. The fourth pressure transducer was an absolute unit used for measuring the freestream static pressure. This transducer had a maximum operating pressure of 15 psia and an accuracy of 0.015 psia. An Omega 100-ohm, 3-wire RTD was mounted underneath the port wing of the O-2A for total-temperature measurements. With the exception of the hotfilm and hotwire cables, all instrumentation channels leading from the model to the DAQboards ran through the leading edge of the O-2A aircraft via 22 GA, shielded, twisted-pair wire. Hotfilm and hotwire cables also ran through the leading edge but utilized RG-174 coaxial cable.

Early in the program, the pilots requested a display of $\beta$ while on test conditions. Initially two displays were added. One screen showed $\alpha$ while the second showed $\beta$. While on test conditions the pilot was expected to hold $\beta$ constant, maintaining a 
constant $A o A$ on the model. $\alpha$ was not as critical and no attempt was made to control $\alpha$ during the dive. As a result later flight tests displayed $R e_{c}$ in lieu of the $\alpha$ display. The two digital screens used for the pilot's display were Otek LPE panel meters. The Otek displays were unique in that they have programmable gains and offsets allowing the user to program a custom sensitivity into the display. The displays were powered by 24 VDC and could accept an analog voltage between \pm 2 VDC. Both displays were mounted on top of the instrument panel replacing the panel normally used for jettisoning the military wing stores in an emergency. Since the SWIFT model was never designed to be jettisoned, this panel location was not being utilized and was ideal for mounting the $R e_{c}$ and $\beta$ displays. In addition, the power switch to turn on the displays also sent power to the pressure transducers for the five-hole probe. This feature gave control to the test pilot for shutting down both AC and DC electrical power in the event of an emergency.

\subsection{Software}

All analog input/output channels were acquired through Labview 8.2 and analyzed with Matlab 6.5. The data-acquisition programs were created in house in order to meet the specific needs for SWIFT. Labview provides a means for embedding Matlab scripts into Labview. The overall strategy was to use Labview for hardware communication and Matlab for any calculations and post processing. Two programs were used during the course of the SWIFT experiments. SWIFT Dive Record.vi is a Labview program designed for acquiring data and sending $R e_{c}$ and $\beta$ signals forward to the digital display for the pilot. During the climb this program was also used to record 
the temperature lapse rate up to altitude as described in Section 5. This program also acquires continuous data during the dive.

The digital video from the IR camera was acquired through the FLIR provided software, ThermaCam Researcher 2001. The ThermaCam software was used without modifications for flight. The software provided both acquisition and real-time display of the IR image. The real-time display proved to be useful as the FTE could watch boundary-layer transition during the dive and call out different test points on the fly.

While on test conditions, both software programs ran on the flight computer simultaneously. Provided the sampling rate was low enough, e.g. (less than $30 \mathrm{~Hz}$ ), both programs could run simultaneously with no issues. At sampling rates above $30 \mathrm{~Hz}$ there were communication problems at the PCMCIA interface. Typically all freestream data with time stamps were recorded at $20 \mathrm{~Hz}$. The IR video data were also sampled at 20 frames per second so that the freestream data and IR video could be merged together to produce a video for each dive. This arrangement proved very helpful when identifying changes in transition and trying to correlate them with changes in roughness or changes in freestream conditions, such as atmospheric turbulence. As a result videos were incorporated for all later flights. Whenever a high-speed acquisition was needed, a buffered acquisition was performed in Labview with the consequence that the pilot's display and IR camera image would freeze momentarily on the laptop. Since most high- 
speed acquisitions only require a one- or two-second acquisition, the frozen screen was never an issue.

A second Labview program IR review.vi was used for all post processing of the data. This program was used for creating a video file of the data combined with the IR images. It could also be used for generating still images of the freestream conditions for any given time during the dive.

\subsection{Freestream Measurements}

During the flying-qualities campaign and initial transition studies a Space Age Controls air data boom was used to record freestream conditions on the starboard wing. It was assumed that the conditions measured on the starboard side of the aircraft would be equivalent to those on the port side and also equivalent to the freestream conditions in front of the aircraft. This was desirable because all instrumentation could be mounted on the starboard wing helping to reduce some of the rolling moment from the heavier SWIFT model on the port wing. Furthermore, with all the instrumentation mounted on the starboard wing, this cleaned up the flowfield on the port wing near the SWIFT model. However, during the initial testing phase it was discovered that the $\beta$ measured on the starboard side of the aircraft was not equal to the $\beta$ measured near the SWIFT model on the port side. It was initially assumed that the outboard pylon position was far enough from the propeller and fuselage that the $\beta$ angle measured on the starboard wing 
would be equivalent to the $\beta$ angle experienced by the SWIFT model. This turned out to be an incorrect assumption.

It was discovered experimentally, and confirmed computationally, that the $\beta$ angle varies between the starboard outboard pylon and the port outboard pylon. As the aircraft moves through the air, the fuselage displaces the air around the front engine cowling. This produces a positive $V$ velocity on the starboard side and a negative $V$ velocity on the port side. $V$ was assumed to be zero at both outboard pylon positions. There were several clues that could have prevented this error, but were ignored early on. Initial designs for placing the air data boom at the outboard pylon location focused on minimizing static and total pressure errors. The air data boom was extended below and forward of the O-2A wing to minimize these errors. However, both $\alpha$ and $\beta$ were never examined when designing the mount for the air data boom, and as a result all initial flights were conducted at incorrect $\alpha$ and $\beta$ values. A more detailed description of the flowfield around the aircraft can be found in Rhodes et al. (2008). In summary, Figure 9 shows a plot of $A o A$ along two lines extending to \pm 20 meters at the two outboard pylon positions. For this simulation a zero $\alpha$, zero $\beta$ condition was input into the freestream, which is characteristic of a dive attitude. Figure 9 shows that $A o A$ differs from the starboard side compared to the port side of the aircraft, where they were initially assumed to be equal and zero for this case. There is nominally a 2-degree difference between the starboard outboard pylon position and the port outboard pylon position. Furthermore, the plot of $A o A$ along the port outboard pylon position shows a large rise 
and fall in $A o A$ near $\mathrm{X}=1 \mathrm{~m}$, which is not matched on the starboard side. This is the influence of the SWIFT model only seen on the port side of the aircraft.

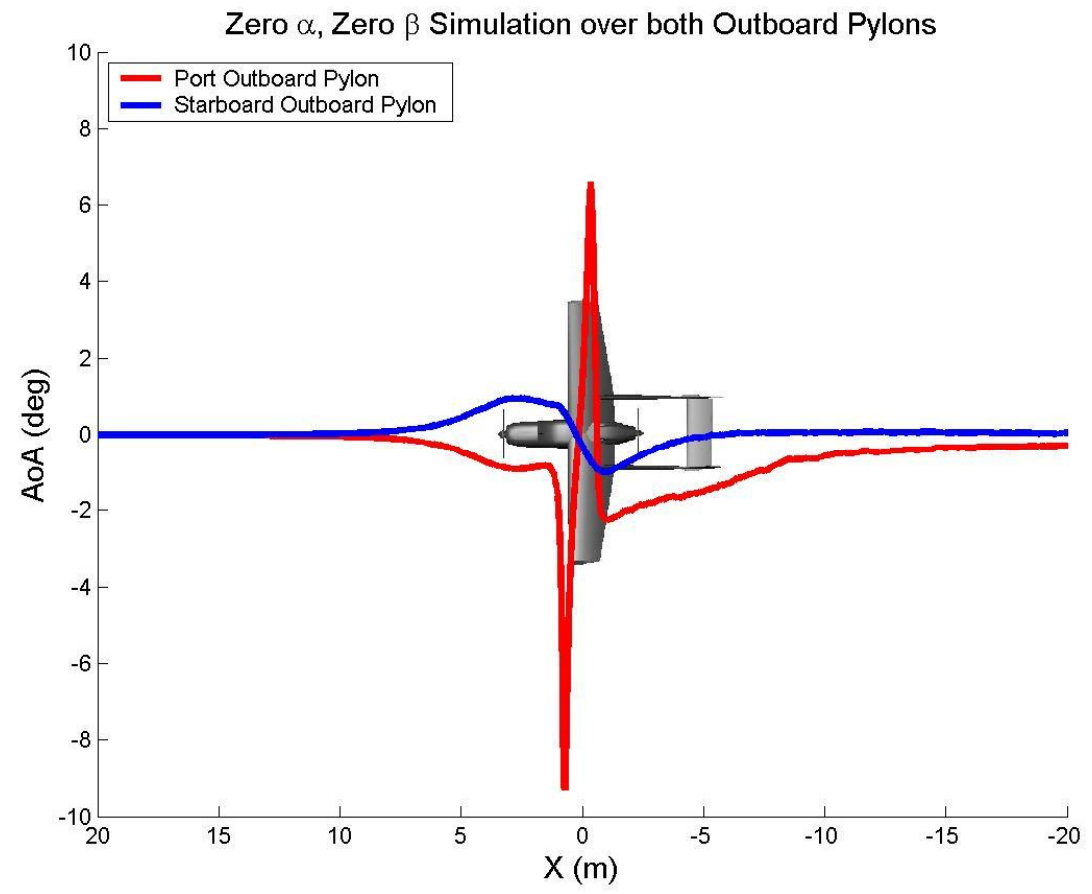

Figure 9: Comparison of $A o A$ for the starboard and port outboard pylon locations.

The discrepancy in $A o A$, displayed in Figure 9, was first discovered by examining the attachment line $C p$ values. The first pressure port read highest pressure, not at zero degrees $A o A$ as one would expect for an almost symmetric airfoil, but rather at 2 degrees $A o A$. This indicated that there was roughly a -2 degree $A o A$ on the model when the air data boom on the starboard wing was measuring zero AoA. To verify this discrepancy a five-hole probe was mounted to the model at half span to make 
measurements locally. A bracket was designed to extend the five-hole probe out into the local freestream, but offset from the SWIFT chordline to insure that the wake of the probe was not in line with the leading edge and testside of the model. This was confirmed by looking at transition images with and without the probe to confirm no changes in transition location. The positioning was also confirmed by calculations from Rhodes et al. (2008) to verify that the probe was mounted forward of the SWIFT static pressure and $A o A$ influence. Moreover, the test points of interest for the SWIFT program were negative AoAs, which had a tendency to push the wake of the five-hole probe away from the testside of the model. The five-hole probe was 24 inches long, but the custom bracket extended the tip of the five-hole probe 25 inches forward of the leading edge and offset 6 inches from the chordline of the model. Figure 10 shows the five-hole probe mounted to the SWIFT model. Simultaneous measurements from the five-hole probe and air data boom confirmed the $\beta / A o A$ discrepancy calculated by Rhodes et al. (2008). As a result, the five-hole probe was implemented for all future flights and the air data boom on the starboard wing was replaced with the hotwire sting mount shown in Figures 6 and 7. 

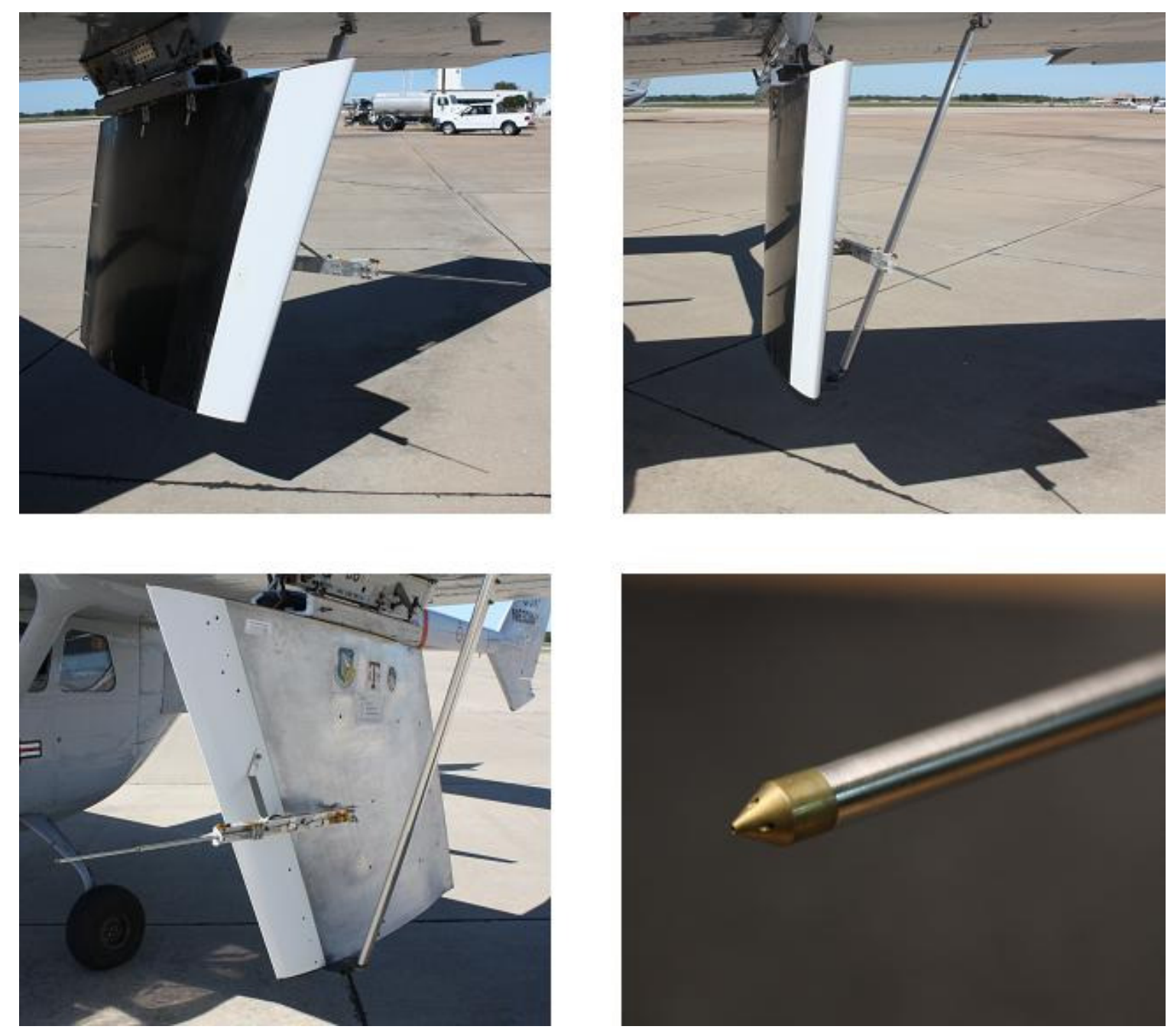

Figure 10: Five-hole probe mounted to SWIFT model.

Figure 9 shows that there is an upstream influence generated by the forward engine cowling. To capture the aircraft freestream conditions at one of the outboard pylon positions would require a probe extended forward of the front propeller. This was not practical, so it was decided to make localized freestream measurements inside the influence of the fuselage while being outside the influence of the SWIFT model. 
Additionally, the calculations performed in Figure 9 do not include the forward engine propeller. Since the $A o A$ comparison between the port and starboard side matched the computational results, there is confidence that the flow angularity is produced by the shape of the fuselage and not the front propeller.

\subsection{Measurement Uncertainty}

After taking into account the uncertainty in all instrumentation, it is instructive to determine the uncertainty in some of the calculated parameters such as airspeed, $R e_{c}, \alpha$, and $\beta$. Both airspeed and $\operatorname{Re}_{c}$ had an uncertainty of $0.2 \%$. The largest source of uncertainty in both $\alpha$ and $\beta$ came from the errors in aligning the five-hole probe and the SWIFT model. A system was devised for aligning the five-hole probe every time it was removed from the model, but despite best efforts to come up with a sound technique for aligning the five-hole probe, the uncertainty is estimated to be \pm 0.2 degree for $A o A$. The uncertainty in $\alpha$ is \pm 0.1 degree. This source of error was much larger than the error associated with instrumentation accuracy for the pressure transducers and the calibration of the five-hole probe, therefore the uncertainty of \pm 0.2 degree is used as a conservative estimate for $A o A$.

Despite only having an accuracy of \pm 0.2 degrees, all $A o A$ values reported here

display two digits of precision. The two digits of precision is used to identify different test points with those test points calculated in Rhodes et al. (2008). Therefore, the reader 
is reminded that a \pm 0.2 degree $A o A$ discrepancy between two figures can be ignored and the two AoAs can be assumed to be equal.

Moreover, the determining factor for calculating the overall measurement uncertainty is not in the uncertainty in the instrumentation, but rather the steadiness of the flight conditions. Despite every effort to avoid atmospheric turbulence, some of the test points were recorded during turbulent conditions. Typically these test points were deleted and repeated. As a result a method was devised for characterizing the turbulence for each test point. Among the information available, simple plots of both $\alpha$ and $\beta$ as a function of time were the best indicators of atmospheric turbulence. As a result, every infrared image displaying transition was accompanied with an $\alpha$ and $\beta$ plot to show the "smoothness" of the conditions. Figure 11 shows an example from Flight No: 111306-2 where smooth conditions were recorded at 5000 feet with very little variation in $\alpha$ and $\beta$ over the five seconds of acquisition. On the other hand, at 3000 feet, atmospheric turbulence was encountered and variations in $\alpha$ and $\beta$ were unacceptable, i.e. on the order of \pm 1 degree. Whenever conditions like these occurred, the dive was terminated and those test points were repeated. 

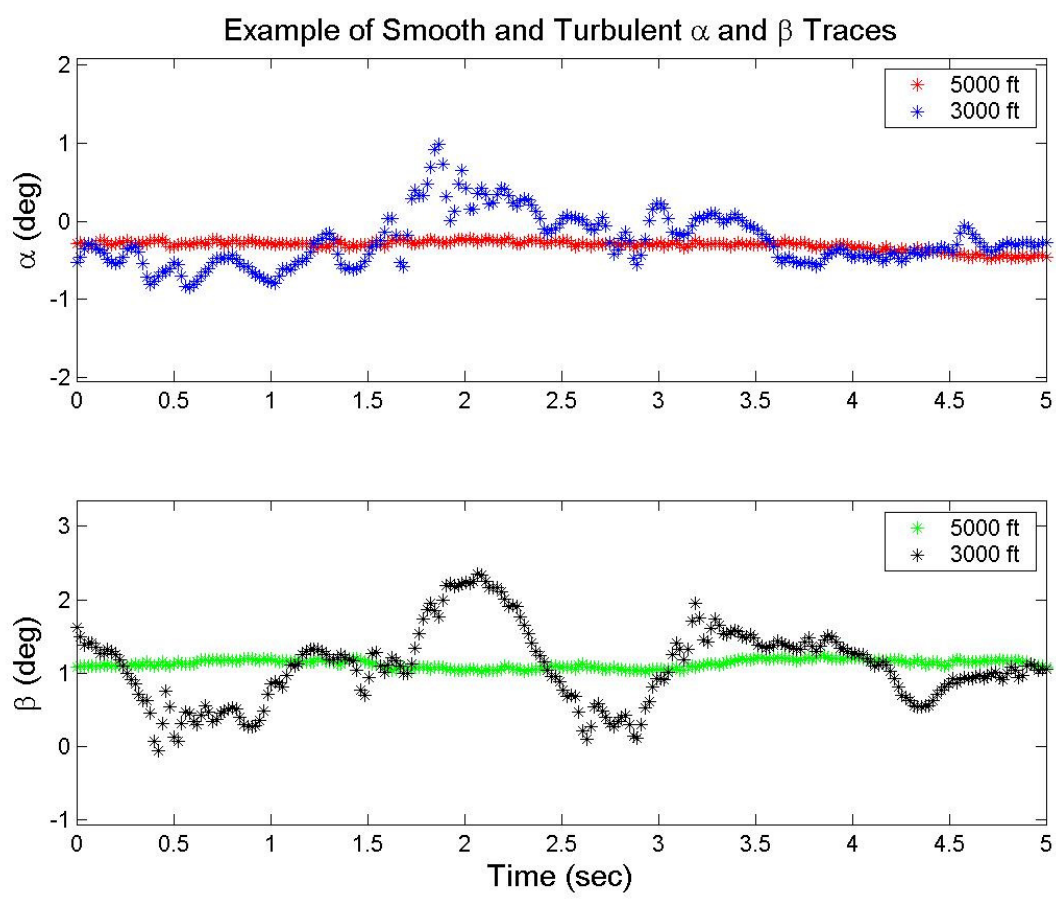

Figure 11: Plots of $\alpha$ and $\beta$ showing both a smooth and a rough test point for the same dive at two different altitudes.

\subsection{Ground Based Instrumentation}

\subsubsection{Surface Roughness Measurements}

A Mitutoyo SJ-400 Surface Roughness Tester was used for measuring the surface roughness of the SWIFT leading edge. The SJ-400 model is a small, hand-held unit designed for taking into the field and making localized measurements. The entire setup is comprised of two pieces: the roughness tester, which includes the stylus used to scan the surface, and the display unit which provides battery power, a digital touchscreen panel for the user, a printer, and serial connections for connecting to a computer. 
The display unit also has a memory-card slot for saving data without a computer. The SJ-400 also comes with a calibrated roughness specimen to allow the user to selfcalibrate the tester whenever needed. Additionally, the SJ-400 has the capability to measure step heights, but was not used during the SWIFT program because a stepcalibration piece was not available.

The SJ-400 has a maximum travel of $25 \mathrm{~mm}$ with the stylus. It was desired to measure the surface roughness across the entire span of the SWIFT leading edge. A jig was designed for moving the roughness tester across the entire span of the model at 25 $\mathrm{mm}$ increments. After making measurements, the roughness tester would be moved approximately 1 inch over and the measurements repeated. This procedure was repeated along the entire span of the leading edge and a statistical average of all the roughness values was used to characterize the entire surface. To facilitate this procedure, the jig was attached to the chuck of a three-axis mill to provide accurate positioning. The leading edge, with its removable design, was placed on the table of the mill for making the measurements. All surface roughness measurements were made as close to the DRE $x / c$ location as possible, which was in the region of $1 \%$ to $2 \% x / c$. Figure 12 shows a picture of the setup on the mill. 


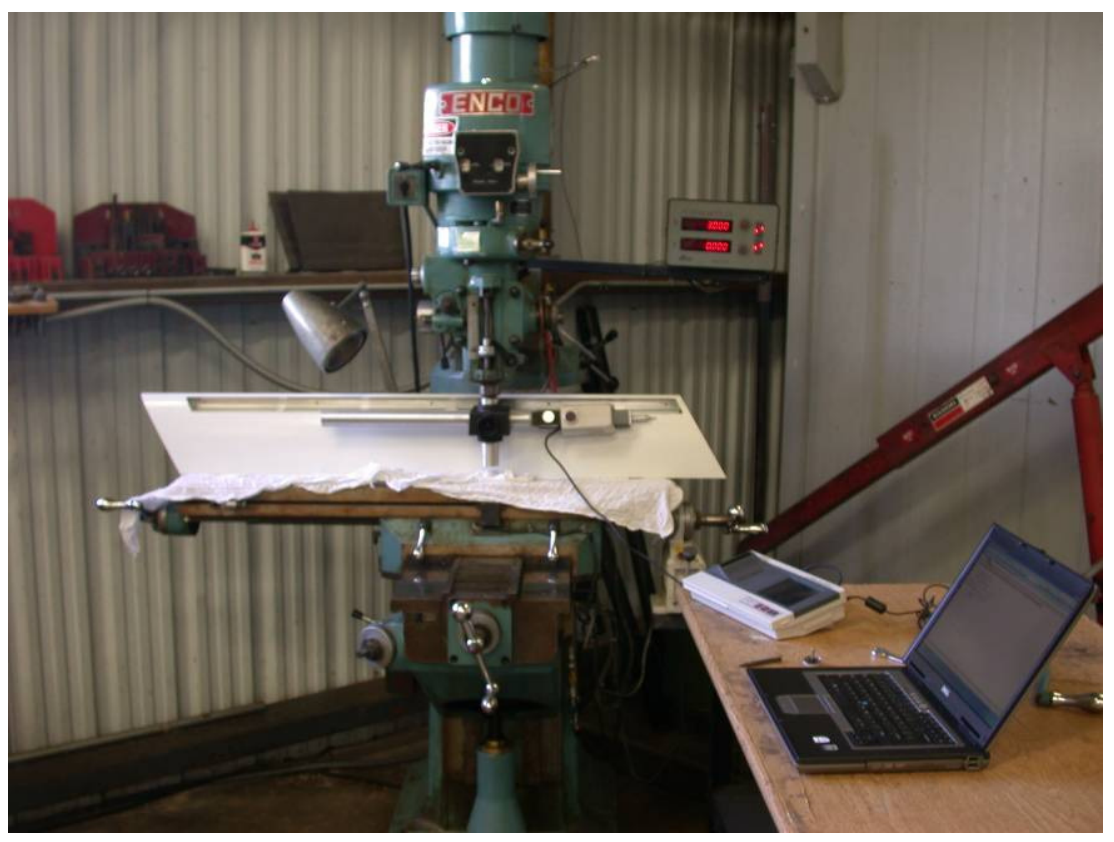

Figure 12: Picture of surface roughness measurements on the SWIFT leading edge.

After scanning the surface, the display unit automatically displays the roughness statistics defined by the user. For these measurements the most useful statistics for characterizing the roughness of the leading edge were the RMS roughness, $P_{q}$, mean of absolute deviations, $P_{a}$, maximum peak-to-peak value, $P_{y}$, and the five-point average of the maximum peak-to-peak values, $P_{z}$. Furthermore, the SJ-400 performed filtering options for looking at specific roughness wavelengths. For example, a low-pass filter could be applied for identifying surface waviness. Alternatively, a high-pass filter could be applied to identify only surface roughness. Because both surface roughness and surface waviness were important for the SWIFT measurements, a P-profile was selected to perform no filtering on the data. The P-profile was used for all SWIFT measurements 
and all measurements used for a comparison. Table 1 shows a list of P-profile statistics as a comparison between different rough surfaces.

Table 1: Surface roughness examples measured at the FRL.

\begin{tabular}{|c|c|c|c|c|c|}
\hline Surface & Material/Coating & $\underline{P q}(\mu \mathrm{m})$ & $\underline{\mathbf{P a}(\mu \mathrm{m})}$ & $\underline{\operatorname{Py}(\mu \mathrm{m})}$ & $\underline{\mathbf{P z}(\mu \mathrm{m})}$ \\
\hline Gulfstream-V Wing & Painted Aluminum & 0.16 & 0.11 & 2.72 & 1.67 \\
\hline SWIFT DRE Insert Leading Edge & Polished Aluminum & 0.24 & 0.19 & 1.46 & 1.00 \\
\hline Learjet 45 Wing & Painted Aluminum & 0.24 & 0.18 & 2.60 & 1.55 \\
\hline $\begin{array}{l}\text { SWIFT Pressure Port Leading } \\
\text { Edge }\end{array}$ & Polished Aluminum & 0.33 & NA & NA & 2.20 \\
\hline $\begin{array}{c}\text { Stemme S-10V Motorglider } \\
\text { Horizontal Tail } \\
\end{array}$ & Painted Carbon Fiber & 0.34 & 0.28 & 2.08 & 1.20 \\
\hline $\begin{array}{c}\text { NLF(2)-0415 Swept-Wing Wind } \\
\text { Tunnel Model }\end{array}$ & Polished Aluminum & 0.38 & 0.32 & 1.81 & 1.36 \\
\hline Tape covered DRE Insert & $\begin{array}{l}\text { Polyester Film Tape over } \\
\text { Polished Aluminum }\end{array}$ & 1.24 & 1.03 & 5.46 & 4.57 \\
\hline Velocity Aircraft Canard & Painted Fiberglass & 1.63 & 1.33 & 7.33 & 6.06 \\
\hline $\begin{array}{l}\text { SWIFT Painted LE (sanded down } \\
\text { with } 1000 \text { grit sandpaper) }\end{array}$ & $\begin{array}{l}\text { Sanded down Painted } \\
\text { Aluminum }\end{array}$ & 1.66 & 1.17 & 12.38 & 7.69 \\
\hline Velocity Aircraft Canard & Primed Fiberglass & 1.75 & 1.39 & 9.25 & 5.97 \\
\hline Cessna O-2A Wing & Painted Aluminum & 1.83 & 1.31 & 9.77 & 7.08 \\
\hline Beech Bonanza Wing & Painted Aluminum & 1.87 & 1.39 & 17.82 & 10.04 \\
\hline $\begin{array}{c}\text { NLF(2)-0415 Swept-Wing Wind } \\
\text { Tunnel Model }\end{array}$ & Painted Aluminum & 1.95 & 1.61 & 8.37 & 5.97 \\
\hline Global Hawk Wing & Painted Carbon Fiber & 1.98 & 1.61 & 9.97 & 6.37 \\
\hline Mooney Wing & Painted Aluminum & 2.05 & 1.76 & 10.52 & 7.64 \\
\hline Global Hawk Landing Gear Door & Painted Carbon Fiber & 4.32 & 3.58 & 23.59 & 17.47 \\
\hline SWIFT Painted LE, unsanded & $\begin{array}{l}\text { Painted Aluminum with no } \\
\text { thinning agent }\end{array}$ & 6.24 & 4.84 & 42.10 & 31.42 \\
\hline
\end{tabular}


For all measurements a range of $80 \mu \mathrm{m}$ was selected resulting in a resolution of $0.00125 \mu \mathrm{m}$. It was routine to recalibrate the device daily before making measurements. Furthermore, one pass was always made over the calibration specimen to check the accuracy of the calibration. This procedure was deemed sufficient for the two-digit precision needed from the measurements. Lastly the stylus presses down with a force of $0.75 \mathrm{mN}$ on the surface, which was light enough to not create any visible scratches in the polished aluminum and painted surfaces tested here.

The SJ-400 was also used to scan over the surface of DREs. The problem with this application is that the stylus of the SJ-400 would drag through the surface of a DRE and remove some of the material. This was observed after looking at the DREs under a microscope. Furthermore, the small pressure applied by the stylus pushed down the tape on the variable-height DREs altering the height of the DRE. Despite these difficulties, the SJ-400 produced very clean scans of DREs and will be presented later in Section 6 to characterize the cross-sectional shape of some of the roughness elements.

\subsubsection{Roughness Height Measurements}

A Keyence LT-8120 confocal laser was used primarily for measuring roughness heights on the variable-height DREs. The confocal laser emits a Class II laser which reflects off the surface of interest and measures the displacement from the reflected light. The laser was also mounted in the thee-axis mill for positioning control within a

resolution of $12.5 \mu \mathrm{m}$. The LT-8120 can be used for measuring displacements, 
maximum peak heights, or maximum valley heights. The resolution of the laser is 0.2 $\mu \mathrm{m}$ and is capable of measuring displacements as high as $2 \mathrm{~mm}$. The output signal can be displayed on a television monitor or to a computer for recording. The LT-8120 was used to provide a calibration for the variable-height DREs. Section 6 discusses the variable-height DREs in more detail.

\subsection{Airport Operations}

The FRL's base of operations is Easterwood Airport in College Station, TX. Texas A\&M University owns the airport so it was a natural fit for these experiments and the FRL. The airport is located less than three miles west of the campus. The airport has three intersecting runways with the longest runway measured at 7,000 feet. In 2007 there was an average of 138 operations per day with a breakdown of $14 \%$ commercial, $30 \%$ military, and 56\% general aviation. These statistics have been updated for 2007 and differ from the statistics in Saric et al. (2006) which were based on older data. The airport was controlled, class-D airspace during the day, but reverted to uncontrolled, class-G airspace during the hours of 2100 to 0800 local time. During most SWIFT operations the airport was class-D controlled airspace unless there was an early morning mission with a takeoff prior to 0800 .

With the exception of the neighboring towns of Bryan and College Station, the airspace was uncongested and mostly farm fields. All missions were conducted either 
due West of the airport from the 280 radial from the College Station VOR, or in the second test area North of Bryan. The North test area called for an initial westbound departure to avoid the congested areas of Bryan, then a turn out to the North once all buildings were cleared. Depending on the weather and sun location, the test pilot decided which test area to fly in.

The FRL occupies a $6000 \mathrm{ft}^{2}$ hangar on the south end of the airport. The hangar contains two temperature-controlled offices on opposite ends of the hangar, occupying approximately $2400 \mathrm{ft}^{2}$ of the internal hangar space. At any given time up to three small aircraft can fit in the hangar utilizing the remaining $3600 \mathrm{ft}^{2}$ of hangar space. Unfortunately, the hangar was not insulated, heated, or cooled so it was subject to all temperature and humidity extremes. All instrumentation not in use would always be stored in one of the temperature-controlled offices at the FRL.

\subsection{Flight Number Documentation}

A flight number is included as a reference in most of the figures. The system created at the FRL is based on the date and the number of the flights conducted that day. For example, the first flight of the day on April 2, 2008 is labeled as Flight No: 0402081. The second flight of the day is labeled as Flight No: 040208-2, and so on. 


\section{TEST CONDITIONS}

\subsection{Motivation}

When conducting an experiment of this nature, it is imperative to document the test conditions as much as possible. Computationalists require initial conditions for simulations and future experimenters will try to recreate promising results. There is never too much data that can be collected, especially data characterizing the freestream. Freestream turbulence, $T u$, is a measure of the flow quality in a wind tunnel or in a flight experiment such as this one. Actually since no effort was made to separate sound from turbulence and to eliminate electronic noise, the numbers quoted below refer to the overall disturbance level. As it turns out, the levels were well below the critical threshold and there was no need to do the separation. Tu is defined in Equation 4 with $u^{\prime}$ the AC fluctuating component of $\mathrm{U}$.

$$
T u=\frac{u^{\prime}}{U}
$$

Crossflow-dominated transition is highly sensitive to freestream turbulence, therefore it is easy to see the importance of mapping out this quantity before one dives into a swept-wing stability experiment. It was hoped that the freestream turbulence measured at the port outboard pylon location would be below $0.1 \%$, low enough to be considered a low-disturbance environment. The experiment could proceed with the design of the SWIFT model provided this requirement was met. 


\subsection{Turbulence Test Procedures}

Prior to the first flight with the SWIFT model, a campaign to measure the freestream-disturbance levels was initiated. A hotwire sting was constructed to be mounted at the same location as the SWIFT model, i.e. the port outboard pylon location. A NACA 0015 sting was constructed to house four freestream hotwires spaced four inches apart. The hotwire sting was smaller than SWIFT, so the bottom hotwire only reached the half-span location of the SWIFT model. The hotwire sting had a span of 23 inches and a chord of 10 inches. It was machined out of aluminum and the entire assembly weighed $32 \mathrm{lbs}$. The hotwire tubes were designed to extend four inches forward of the sting leading edge. Figure 13 shows a picture of the hotwire sting mounted in the SWIFT location and the air data boom attached to the starboard outboard pylon. These measurements were made early in the campaign. As a result the AoA discrepancy measured at the starboard outboard pylon location with the air data boom, as discussed in Section 2.9, had not been discovered yet. However, the air-data-boom's ability to accurately measure true airspeed was not compromised because the mount was specifically designed to eliminate static and total pressure errors. As a result the true airspeed calculations for these measurements were accurate. The measurements of $\alpha$ and $\beta$ were not, but are not important for the determination of $T u$. In Figure 13 the hotwire sting has rubber caps over the four supporting tubes to keep debris out. The bottom of the sting has an RTD extending three inches into the freestream for total-temperature measurements. The internal cavity of the hotwire sting was hollow and some of the early flights included a tri-axial accelerometer. Also depicted in Figure 13 is the air data boom 
used for measurements of freestream conditions. Visible in this figure is a hotwire sting extending below the air data boom. This hotwire sting was a retrofit to the original design of the air-data-boom mount, and was incorporated to also measure turbulence levels. However, this feature of the air data boom was never used as it was replaced with the five-hole probe before hotwires could be attached. This sting also had a RTD extending out the bottom of the sting for total temperature measurements.
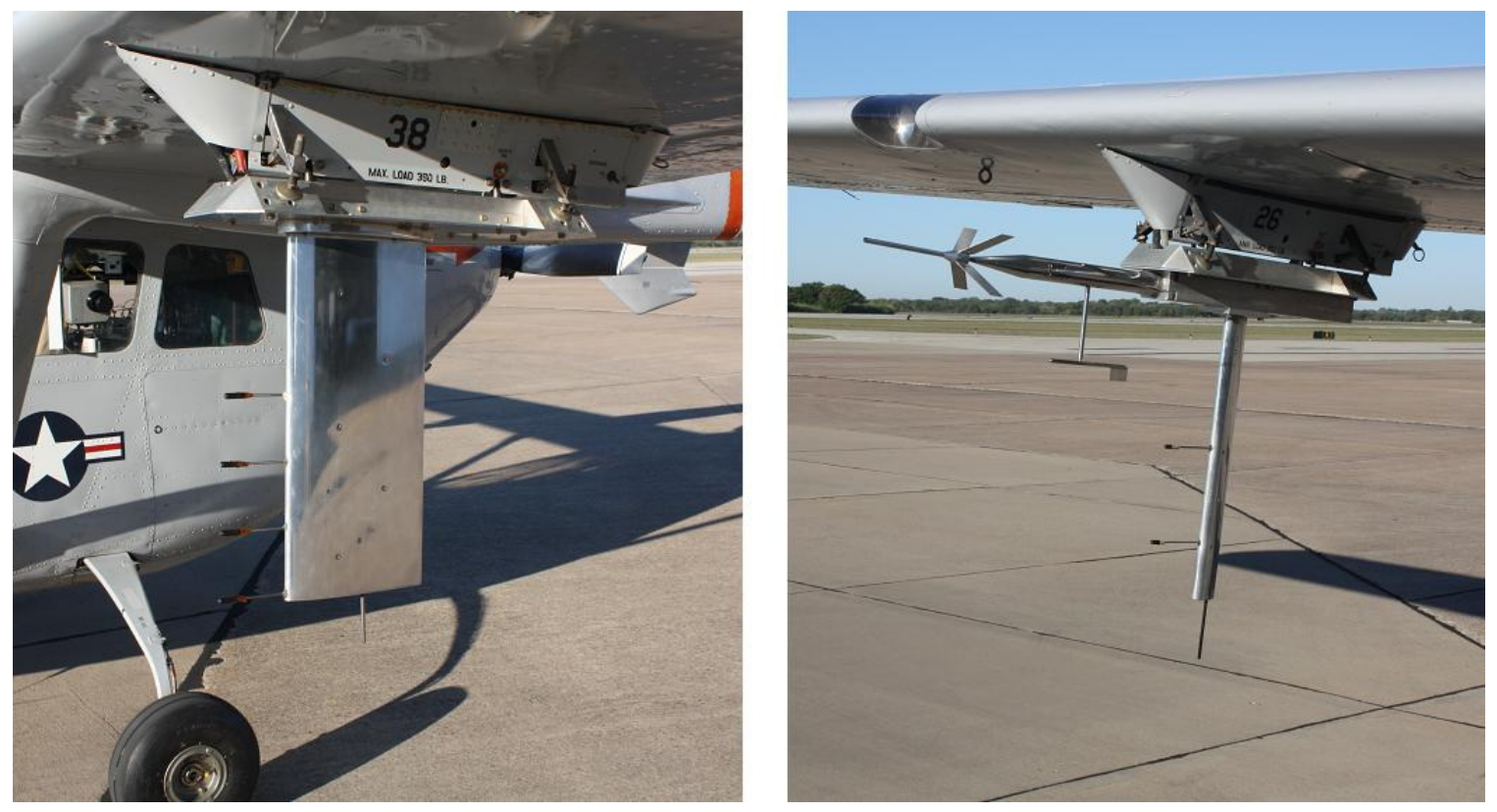

Figure 13: Hotwire sting mounted to the port outboard pylon and air data boom mounted to the starboard outboard pylon.

Two Dantec, $5 \mu \mathrm{m}$, platinum-plated, tungsten hotwires were used for all measurements of $T u$. The two hotwires occupied the bottom two hotwire positions on the sting. The top two locations were never used during these measurements. Both hotwires were connected to an AA Lab Systems AN1003 Constant Temperature Anemometer 
installed in the cabin of the O-2A aircraft. Due to the high weight of the anemometer, 50 lbs per 10 channels, an instrument rack was constructed to shift the anemometer as far forward as possible behind the pilot's seat. The anemometer was powered by 115 VAC provided by the onboard inverter. During this part of the program onboard batteries were used to power all experimental instrumentation by supplying power to the DC to AC inverter. Signal conditioning for both hotwires came from a Kemo VBF44 bandpass filter. Each hotwire signal was bandpassed between $1 \mathrm{~Hz}$ and $10 \mathrm{kHz}$ with both signals sampled at $25 \mathrm{kHz}$.

Turbulence measurements were recorded in the dive at four different altitudes. Because the freestream temperature was constantly increasing during the dive, it was important to take into account temperature effects between the calibration temperature and the temperature the high-speed recordings were made at. To take this into account, four different calibrations were performed for four different altitudes. Calibrations were performed at 3000, 4000, 5000, and 6000 feet in a level attitude. In the level attitude the O-2A tops out at $130 \mathrm{KTAS}$. During the dive, the test pilots typically flew close to 165 $K T A S$. As a result, the calibration curves had to be extrapolated to the higher dive velocity while making turbulence measurements in the dive. Furthermore, the time between calibration and actual high-speed acquisition for $T u$ was as much as one hour for the lowest altitude of 3000 feet. The procedure for this mission was to climb to 3000 feet and perform a calibration by increasing the airspeed at five-knot increments to the aircraft's top speed at that altitude. Then a climb was initiated up to 4000 feet and the 
calibration procedure was repeated at 4000, 5000, and 6000 feet. It was approximately 1 hour between the time it took to perform the first calibration at 3000 feet before disturbance measurements were recorded in the dive back at 3000 feet at the bottom of the dive. During this time the temperature was constantly increasing at all altitudes as the day got longer. However, during the course of this one hour the highest temperature difference between the calibrated temperature and the temperature recorded on the way back down during the dive was only $1{ }^{\circ} \mathrm{C}$. This procedure was considered to be good enough to sample the freestream disturbance levels. Improved techniques for performing temperature compensation with hotwires can be found in Radeztsky et al. (1993b) and White (2000) and should be used for all future measurements of $T u$.

\subsection{Freestream Disturbance Results}

During the dive the freestream disturbance level was measured to be $0.05 \%$ to $0.07 \%$ of $\mathrm{U}$ at airspeeds between 158 and 168 KTAS. These values are low enough to be considered a low-disturbance environment and the experiment was given the green light to proceed into the second phase of the program.

\subsection{SWIFT Accelerometer Data}

A PCB 356A32 tri-axial accelerometer was added to the internal cavity of the SWIFT model. The accelerometer was oriented to measure accelerations in the streamwise $X$, wall normal $Y$, and spanwise $Z$ directions. The 356A32 has a sensitivity of 
$100 \mathrm{mV} / \mathrm{g}$ and can sense accelerations from 2 to $4000 \mathrm{~Hz}$. It was desired to first measure the natural frequency of the model with a simple rap test. A rubber mallet was used to strike the non test side of the model and the flight computer measured the natural frequency of the model in the wall normal direction. Figure 14 shows power spectra from the ground rap test. A natural frequency of approximately $170 \mathrm{~Hz}$ is picked up with the tri-axial accelerometer. The SWIFT model is much stiffer in the streamwise and spanwise directions and an impulsive signal was never recorded after attempting a spanwise rap with the mallet. The streamwise test was never performed to avoid striking the leading edge with the mallet for obvious reasons.

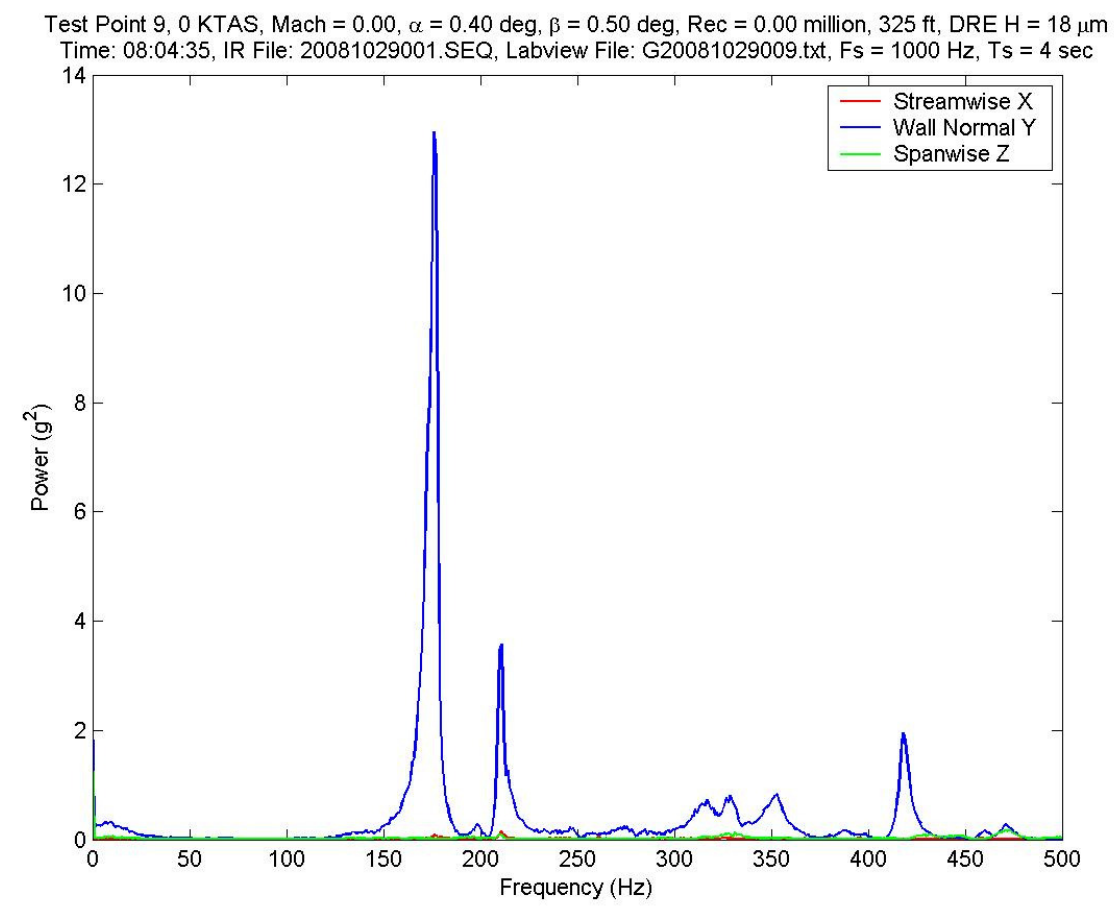

Figure 14: Accelerometer power spectra for the ground rap test. 
It was also desired to measure power spectra with the accelerometer during flight in both the level and dive attitude. The intent here was to verify that the dominate frequencies of vibration during flight did not overlap the T-S passband frequencies. Figure 15 shows power spectra during level flight. In this figure the $170 \mathrm{~Hz}$ natural frequency is present, but so are two other dominate frequencies around $540 \mathrm{~Hz}$ and 1450 Hz. These two higher frequencies come and go as the airspeed is modulated, but no trend appears in the data leading to the source of the vibrations. During the dive, the accelerometer picks up a richer spectrum of frequencies, but only the natural frequency of $170 \mathrm{~Hz}$ appears to dominate. Furthermore, all frequencies start to die off around 1500 Hz. Figure 16 shows the spectra during a typical dive.

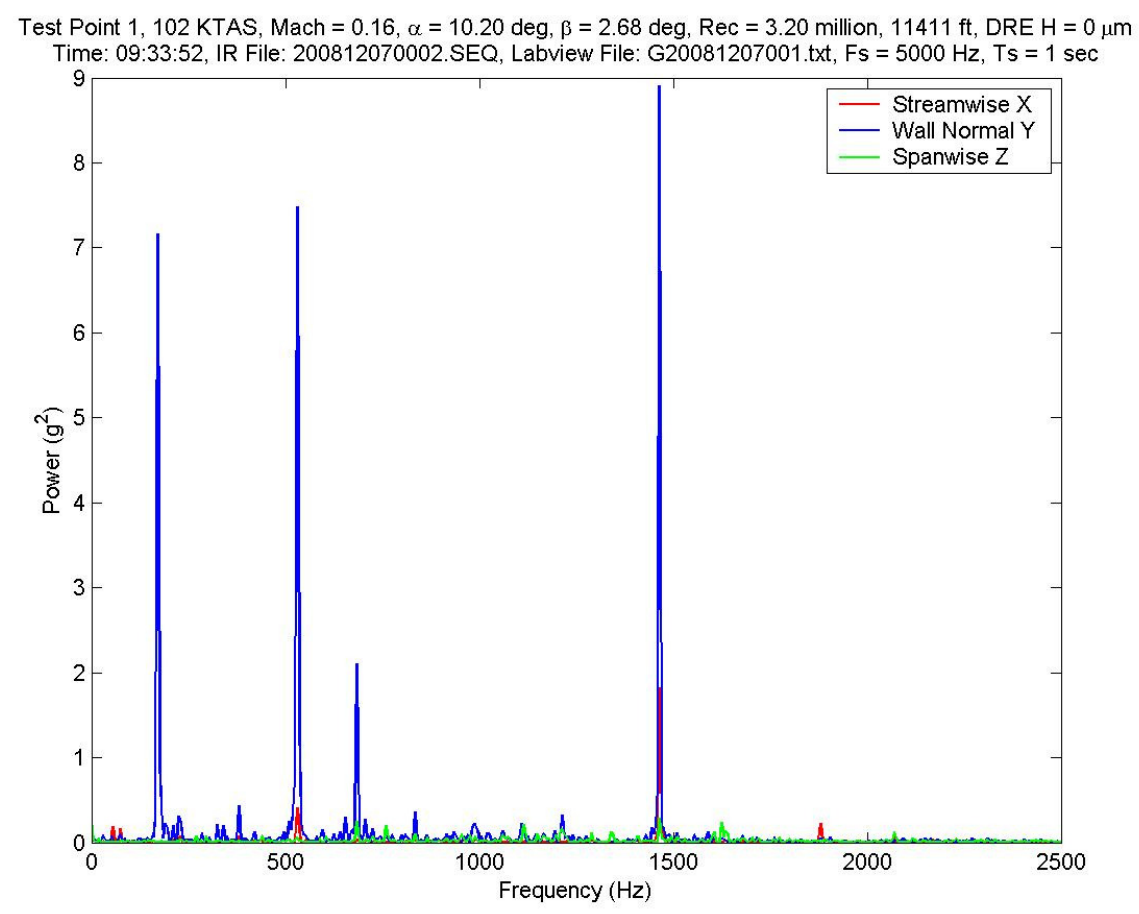

Figure 15: Accelerometer power spectra recorded in flight in a level, cruise attitude. 


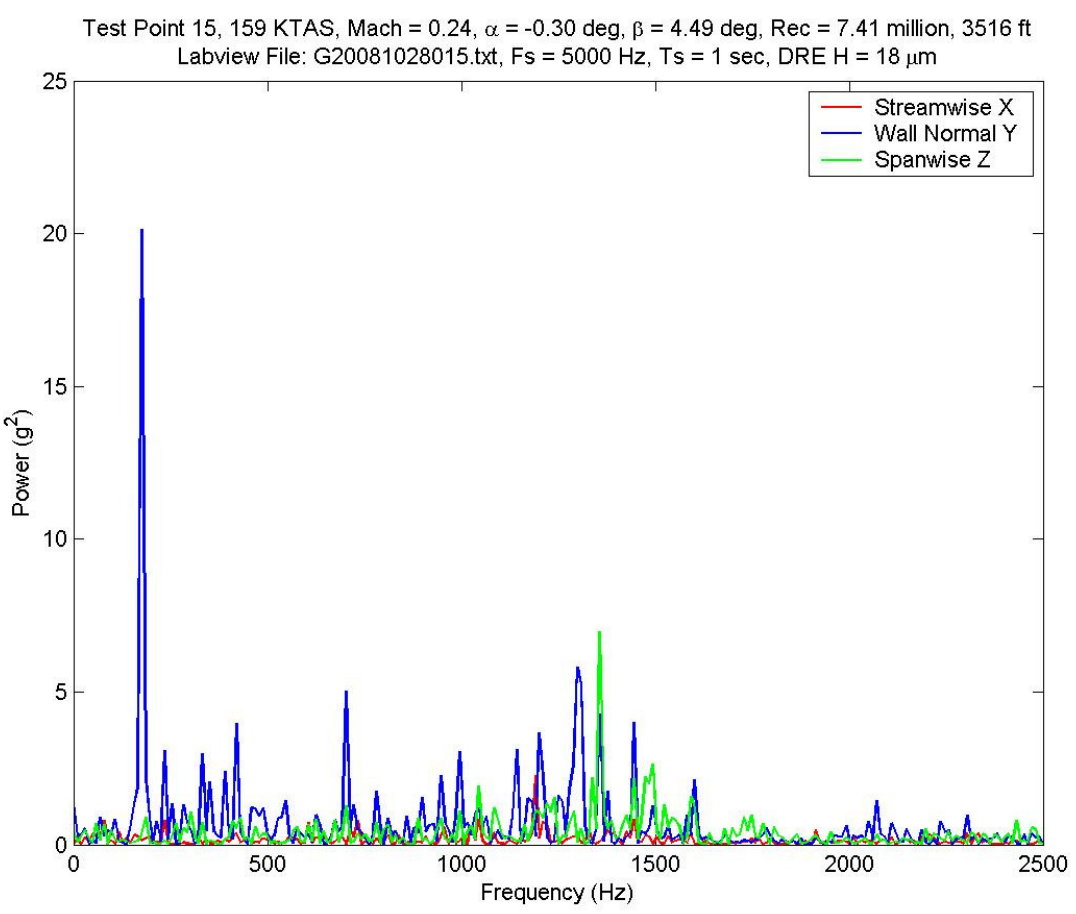

Figure 16: Accelerometer power spectra in the dive attitude.

\subsection{Sound Pressure Levels}

Based on the experiments of Radeztsky et al. (1993a), the crossflow vortices tested there were insensitive to freestream sound up to $125 \mathrm{~dB}$ over all important frequencies. The B-757 wing-glove flight tests also demonstrated an insensitivity to sound with sound-pressure levels as high as $130 \mathrm{~dB}$ generated by high-bypass ratio engines (Runyan et al. 1987). It was desired to record the freestream sound-pressure levels from the engines of the O-2A aircraft during flight with a PCB-style microphone. However, this was not completed in time so this task is left to future experimenters. Currently there does not appear to be any sensitivity to engine noise. 


\section{4. $C p$ MEASUREMENTS}

The SWIFT program consisted of both a computational effort and an experimental effort. The experimental part is described here whereas the computational effort is summarized in Rhodes et al. (2008). Moreover, Dr. Helen Reed and her coworkers devoted considerable efforts over the past four years in the basic airfoil design and the associated boundary-layer stability calculations. Rhodes et al. (2008) and Carpenter et al. (2009) only touch on history of this work. Rhodes developed a solid model of the aircraft with the SWIFT test article and performed a full Navier-Stokes analysis on the entire flowfield. The primary objective of this computational analysis was to compare the $C p$ measurements recorded in flight with the calculated NavierStokes solution. Once these matched, within experimental error, then the computations proceeded to provide boundary-layer and stability calculations for the experiments. The primary objective for conducting experimental $C p$ measurements on the SWIFT model was to determine which AoAs produced sufficient crossflow, resulting in a crossflowdominated transition. An aft pressure minimum of $70 \% x / c$ with an accelerated flow back to this point was the desired test point for studying the DREs effect on transition. Secondarily, one could compare the experimental $C p$ with the CFD solution to confirm the accuracy of the computational model, and that the mean flow calculations are correct. 


\subsection{Cp Measurement Procedures}

The SWIFT model contains 64 static pressure ports on the surface. The testside of the model has the majority of the ports with 50 total ports divided between two rows. The first row is labeled as the root pressure port row and is located 13 inches below the top of the model. The tip pressure port row is 29 inches below the top of the model. This leaves a 16-inch region without pressure ports in the center of the model, designed to be the area of focus for the transition studies. The leading edge has the highest density of ports with most ports concentrated near the attachment line of the leading edge. Leading edge \#1 was used for all $C p$ measurements as it contained the leading edge pressure ports. All static pressure ports were 0.025 inch inner-diameter stainless-steel tubes. All pressure lines leading back to an internally mounted 64-channel pressure scanner were 0.043 inch inner diameter polyurethane tubing. The pressure scanner was a \pm 5 psid Pressure Systems scanner with 16 bits of data conversion and an accuracy of 0.005 psid. The scanner was imbedded inside the model to reduce pressure lag time within the tubing. Furthermore, it was easier to transmit the electrical signal back to the flight computer with one 15 -conductor wire then to run 64 individual pressure lines back to a cockpit-mounted pressure scanner. For all $C p$ flights the pressure scanner used the freestream static pressure from the five-hole probe as the reference. Figure 17 shows the two pressure port rows in addition to the distribution of ports along the chordline of the model with the highest density near the attachment line. 


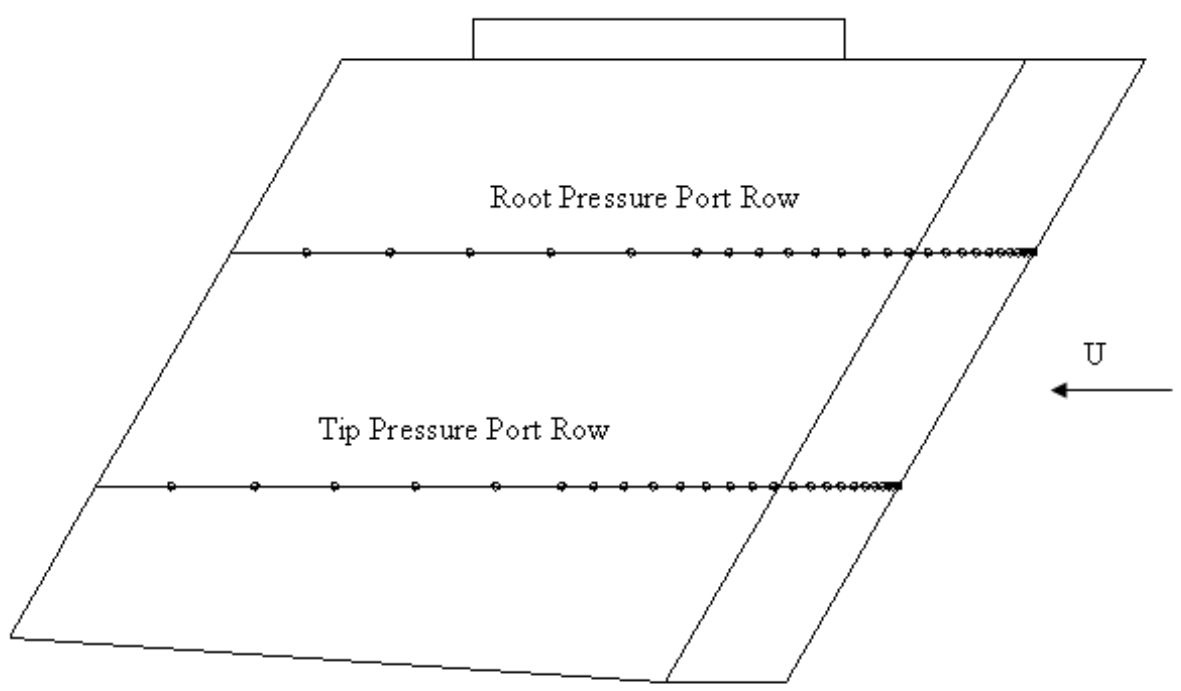

Note: Pressure Ports not Drawn to Scale

Figure 17: Schematic of SWIFT model showing both root and tip pressure port rows.

During all $C p$ measurements the infrared camera was simultaneously used to record transition data. The primary purpose of running the infrared camera in conjunction with the pressure scanner was to make sure the pressure ports near the leading edge were not tripping the boundary layer and producing a turbulent wedge over all ports. It was discovered that above 7.2 million $R e_{c}$, the static pressure ports near the attachment line produced turbulent wedges. This effect was especially problematic during negative AoAs where the sensitivity to the pressure ports increased due to the elevated crossflow and sensitivity to leading edge 3-D disturbances (Radeztsky et al. 1993a). As a result all $C p$ measurements were performed below 7.2 million $R e_{c}$. Of secondary importance, the infrared camera could identify turbulent wedges produced by bug strikes or any other disturbance picked up on the leading edge during takeoff. For 
this case the flight would be terminated so that the leading edge could be wiped clean on the ground. Figure 18 shows an infrared image of turbulent wedges produced by the leading edge pressure ports. The warmer region indicates a turbulent boundary layer, while the colder region outlines a laminar boundary layer. A more detailed explanation of infrared images is given in Section 5. A third turbulent wedge is visible in Figure 18. This turbulent wedge was produced by an isolated roughness element designed to trip the boundary layer. This technique was used to calibrate the infrared images and will also be explained in Section 5 .

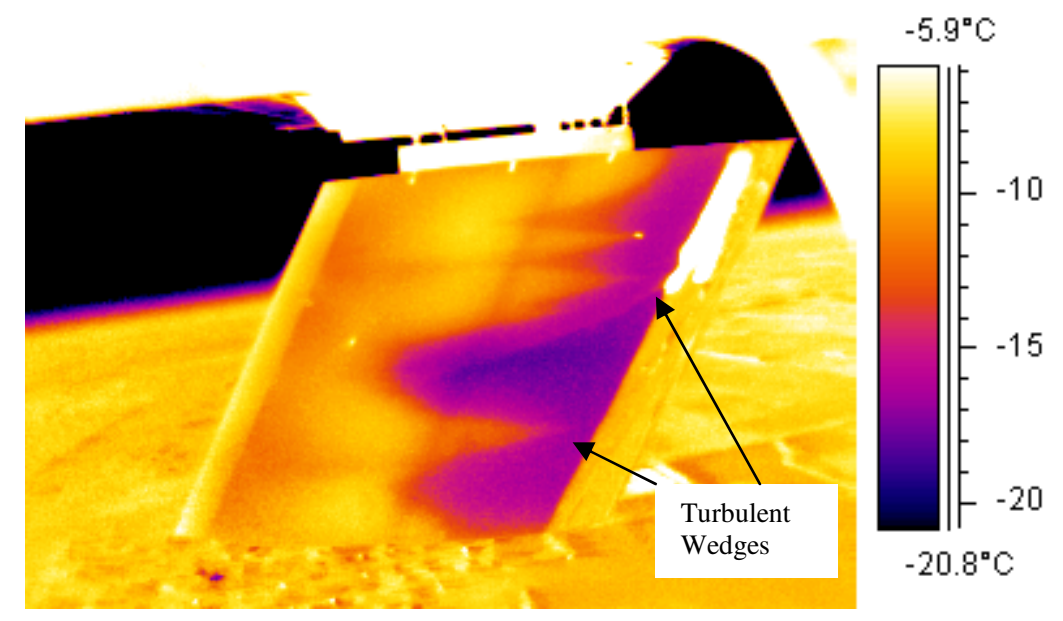

Figure 18: Infrared image showing two turbulent wedges produced by the leading-edge pressure ports and one wedge produced by an isolated roughness element.

The pilot used both the real-time $R e_{c}$ and $\beta$ display during these measurements to maintain conditions as close as possible to the targeted values. During smooth atmospheric conditions the pilot could routinely hold $\beta$ to within \pm 0.1 degrees for a fivesecond pressure acquisition. For each test point a five-second acquisition of freestream 
conditions and $C p$ values was recorded. These values were then averaged over the five seconds, but the scatter of $C p$ values was used to generate error bars to compare with the CFD calculations. The goal was to measure $C p$ values in the dive attitude at $A o A$ values between -5 degrees and +2 degrees at 0.5 degree increments. Figures 19 and 20 show $C p$ values for both the root and tip pressure port rows for three representative cases. Also displayed are the coordinates of the SWIFT airfoil.

The test points selected in Figures 19 and 20 represent the highest, the middle, and one of the lowest $A o A$ values tested during this campaign. Test Point 5, at 2.21 degrees $A o A$, was the highest $A o A$ recorded because a suction peak started to form at the tip pressure port row near the leading edge. The suction peak and overall flat $C p$ curve at this test point was not desired for demonstrating the DREs, so this $A o A$ became the stopping point. Again the goal was to excite crossflow and the flat top of the $C p$ curve at this $A o A$ was not sufficient to meet this goal. Test Point 35, at -1.20 degrees $A o A$, represents the middle $A o A$ tested here. This test point also did not produce enough crossflow and was not tested later in the program. Test Point 35 is only displayed here to show an example of the $C p$ trend as the $A o A$ is decreased for this airfoil. Lastly, Test Point 27, at -4.69 degrees $A o A$, represents one of the lowest $A o A s$ tested and much attention was paid to this test point. This test point demonstrates early transition, and stability calculations showed strong crossflow growth at this $A o A$. What is uniform about all three test points and both pressure port rows displayed in Figures 19 and 20 is that the pressure minimum is near $70 \% x / c$ and all test points show an accelerated favorable 
pressure gradient back to this point. Overall the shape of each $C p$ curve was promising and expected based on the initial airfoil design goals.

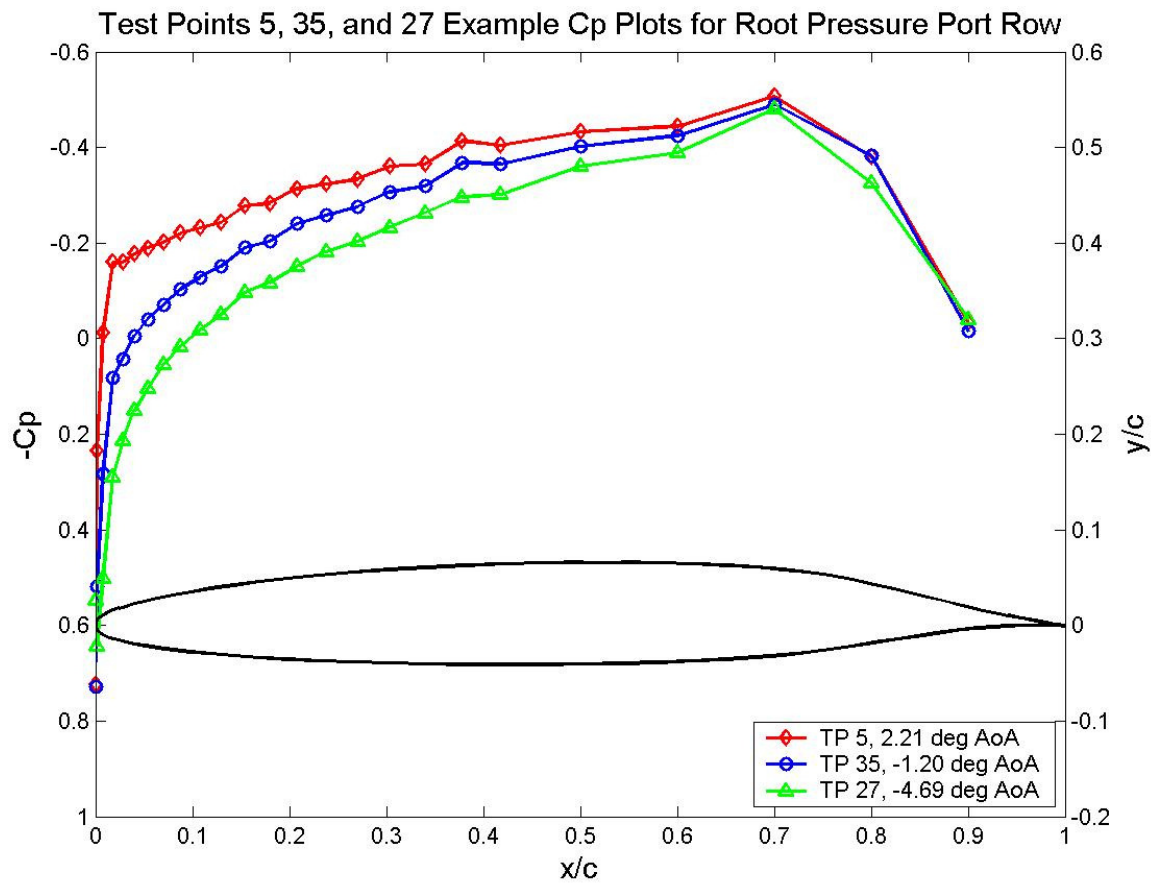

Figure 19: $C p$ data for three representative test points at the root pressure port row. 


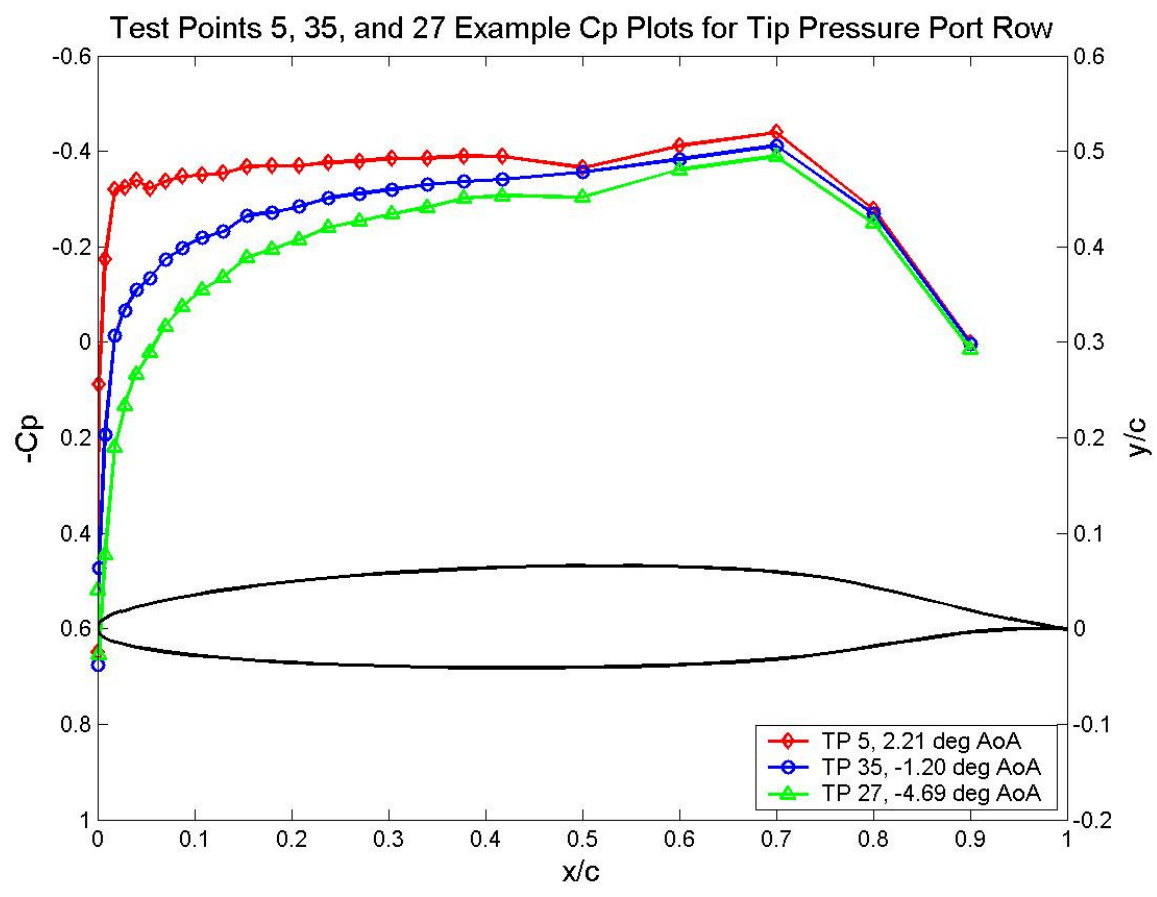

Figure 20: $C p$ data for three representative test points at the tip pressure port row.

The initial goal was to record $C p$ values at 0.5 degree $A o A$ increments. At the end of the $C p$ campaign, 29 test points were recorded between 2.21 degrees $A o A$ and -5.15 degrees $A o A$ resulting in a much finer spacing in $C p$ values. This dissertation focuses on two test points of interest, namely -2.61 and -4.69 degrees $A o A$.

In between the range of -1.01 degrees $\leq A o A \leq 2.21$ degrees the model was mounted at zero incidence where the chordline of the model was parallel to the aircraft centerline. To investigate lower $A o A$ values the model was rotated 1 degree toe out (leading edge rotated away from the fuselage). As a result the aircraft flew relatively straight but still produced negative AoAs on the model. This feature of the SWIFT model 
was designed into the mounting structure and is described in McKnight (2006). This proved to be a good design feature because during one flight the model was kept at zero incidence and the aircraft was set in an extreme sideslip angle to produce positive $A o A s$ on the model. As the pilot rolled the left wing down to maintain a constant heading, the fuel in the port wing rushed to the wingtip and the forward engine quit. After performing a successful single-engine landing it was decided not to exceed $3 / 4$ ball deflection on the aircraft inclinometer. Instead the model would be rotated near the required $A o A$ for all future testing. Once the model was fixed, the pilot still had rudder trim authority to change the $A o A$ on the model by as much as \pm 2 degrees in either direction, provided the inclinometer did not exceed the $3 / 4$ ball deflection self-imposed limit. The most extreme test point was recorded at -5.15 degrees $A o A$. This test point required a two-degree toeout incidence, and is not plotted in Figures 19 and 20 because a computational grid has not been generated for the two-degree toe-out condition to compare with the CFD. The two test points of interest in this dissertation were recorded with the model rotated 1degree toe out, hence this condition was kept for the remainder of the program. Lastly, the change in model incidence could not be done during flight. The mounting structure could not be rotated with the weight of the model attached to it. To change the incidence, the model was removed from the aircraft, placed on a work table, and the mounting structure was rotated to the desired angle. Typically half a day was required to perform this change, but this option proved to be an invaluable feature after experiencing the engine-out incident. 
In all cases it was preferred to use the rudder trim wheel rather than the rudder pedals to control aircraft sideslip. This proved to be more consistent and produced good $A o A$ traces during smooth atmospheric conditions. Only under extreme $A o A$ conditions was it necessary for the pilot to use the rudder pedals.

After completing the $C p$ measurements, it was clear that $\alpha$ does not change significantly. The procedure was to dive at $170 \mathrm{KIAS}$ until the targeted $R e_{c}$ was reached. 170 KIAS typically corresponded to a descent rate around 1500 to 2000 feet per minute. At this attitude $\alpha$ was approximately constant at -0.4 degrees. Using Equation 2 this corresponds to $\Lambda_{L E}=30.4$ degrees. Over the course of the 29 test points tested for $C p$ measurements the $\Lambda_{L E}$ varied from 30.9 degrees at the lowest $A o A$ and 30 degrees at the highest $A o A$. Note that $\alpha$ measured by the five-hole probe is different than the aircraft angle of attack. The aircraft angle of attack is approximately two degrees greater than $\alpha$ because the five-hole probe is installed parallel to the SWIFT chordline and not the O2A chordline.

\subsection{Comparison with CFD}

Two test points were selected for comparing with the CFD solutions, Test Point 31 at -2.61 degrees $A o A$ and Test Point 27 at -4.69 degrees $A o A$. Test Point 31 represents a stable, laminar condition. This $A o A$ was used to demonstrate the critically-spaced DREs in later flights, where the intent was to excite the crossflow instability with DREs and demonstrate early transition on the model. The second test point of interest was a 
less stable case at -4.69 degrees $A o A$. At this point the crossflow is destabilized and signs of early transition appear on the model. This test point was used to demonstrate the DRE control scheme in later flights, where the goal was to increase the extent of laminar flow. Figures 21 through 24 show a comparison between the experimental and computational $C p$ for these two test points and both pressure port rows. The experimental data are represented with error bars generated from the scatter of $C p$ values recorded during the five-second acquisition. Error bars were also generated based on the accuracy of all the instruments, however these error bars proved to be much smaller than the scatter and were not included.

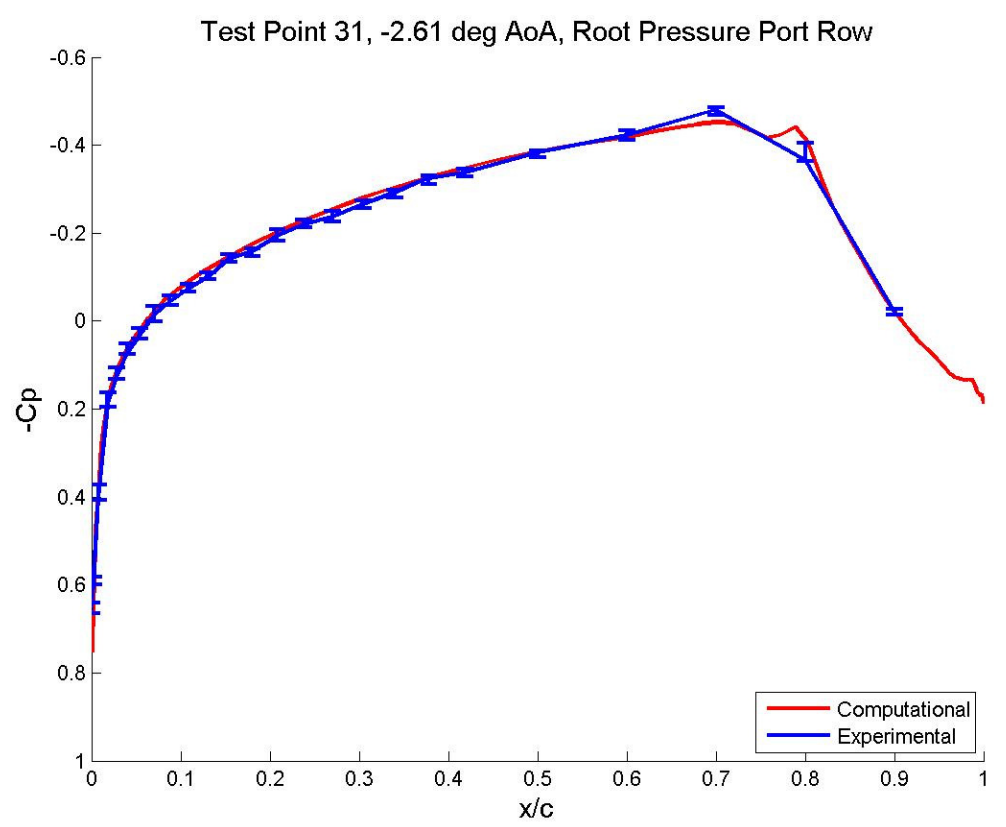

Figure 21: Experimental and computational comparison at -2.61 degrees $A o A$ for the root pressure port row. 


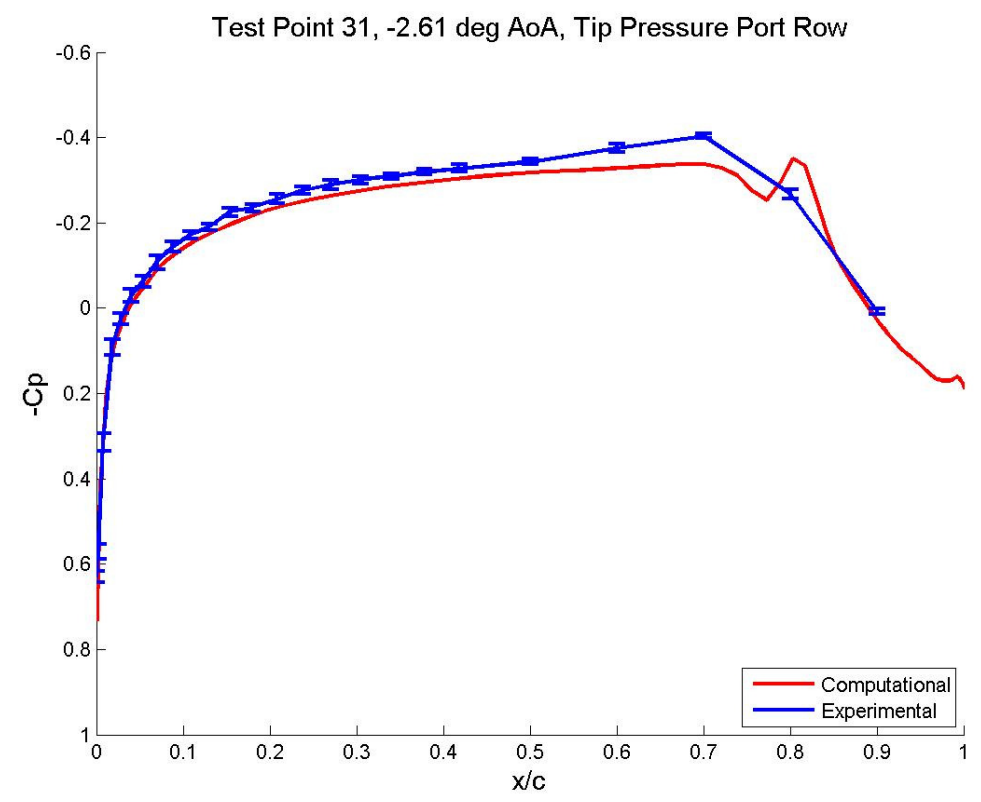

Figure 22: Experimental and computational comparison at -2.61 degrees $A o A$ for the tip pressure port row.

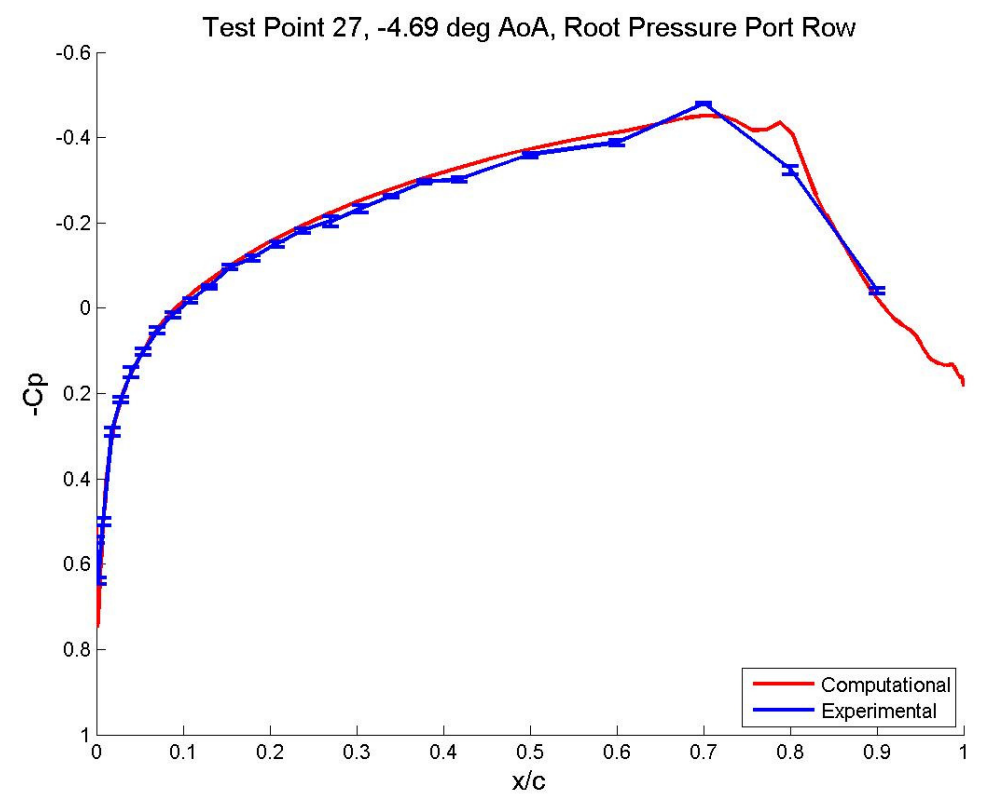

Figure 23: Experimental and computational comparison at -4.69 degrees $A o A$ for the root pressure port row. 


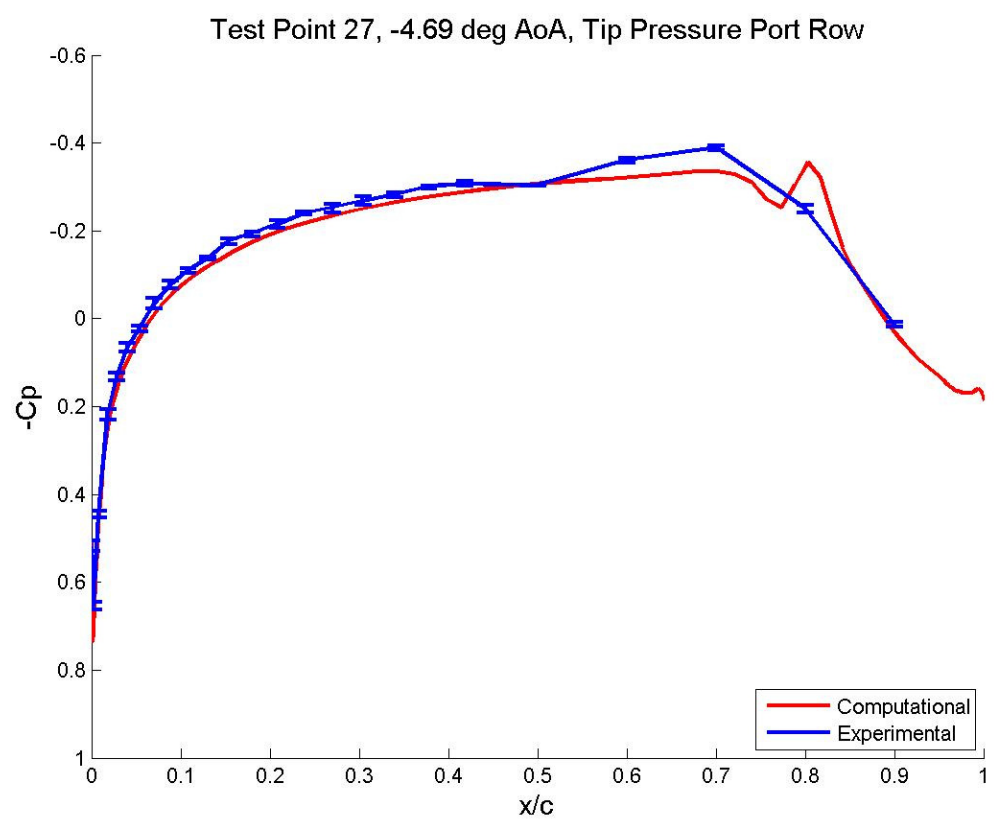

Figure 24: Experimental and computational comparison at -4.69 degrees $A o A$ for the tip pressure port row.

Agreement between the experimental and computational $C p$ values was achieved by taking an iterative approach to match up the $\beta$ values recorded by the five-hole probe with those calculated in the CFD. For both Test Points, 31 and 27, there is similarity at the root pressure port row. The tip pressure port row is close, but not as good as the root. It is believed that the four-degree cut aft of the leading edge at the tip of the model is affecting the computations resulting in some error. Furthermore, there could be some end effects from a wingtip vortex that is affecting the tip pressure measurements. Despite the slight error at the tip pressure port row, excellent agreement was achieved for these two test points and both pressure port rows. 
In Figures 21 through 24 each of the computational $C p$ curves show an oscillation aft of the pressure minimum. This is due to laminar separation in the computations. This effect is not a concern because the stability calculations only use $C p$ values up to the pressure minimum and this laminar separation region is not included. The flight $C p$ curves show a smooth curve aft of the pressure minimum indicating that separation does not occur in flight.

For all $C p$ values, the freestream static pressure and freestream dynamic pressure recorded by the five-hole probe were used as the reference values. The procedure for eliminating turbulent test conditions, outlined in Section 2.10, was used here to generate finalized plots with steady $\alpha$ and $\beta$ traces within \pm 0.1 degrees. Lastly, after reviewing Figures 21 through 24 one could conclude that the channel attached to the tip pressure port row at $50 \% x / c$ is bad and should be deleted from all $C p$ curves. This channel was found to be intermittent because the dip in $C p$ values is not seen in all test points. As a result, this pressure port remains in all the $C p$ figures.

\subsection{Root and Tip Pressure Port Comparison}

A comparison between the root and tip pressure port rows can be made with the experimental data. The SWIFT model exhibits an obvious spanwise variation in the $C p$ data. The tip pressure port row accelerates sooner, but does not reach the same pressure minimum as the root pressure port row. The tip pressure port row also exhibits a more obvious suction peak near the leading edge and positive AoAs. Figures 25 and 26 show a 
comparison between the root and tip pressure port rows for both Test Points 31 and 27 . The experiments of Radeztsky et al. (1993a), Reibert et al. (1996), Chapman et al. (1998), Saric et al. (1998), and White \& Saric (2000) all incorporated wall liners at the top and bottom of the model to generate a spanwise uniform flow. This option was not available for the SWIFT model so all transition studies take into account this spanwise variation on the model.

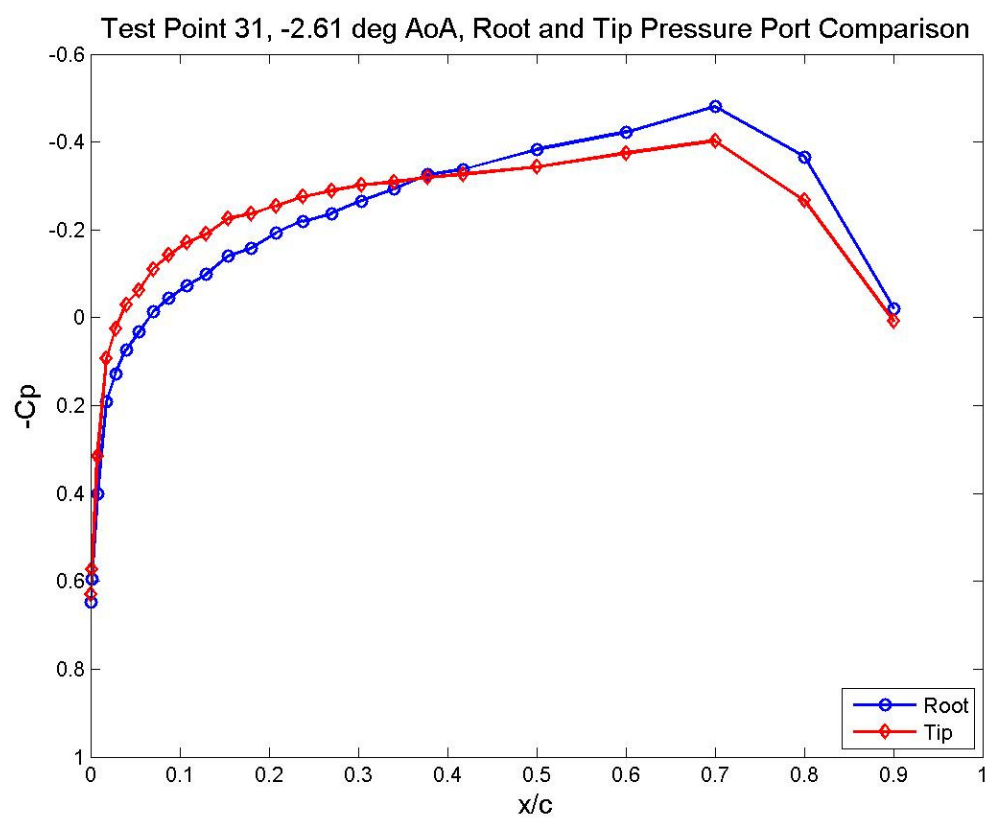

Figure 25: Root and tip pressure port comparison for Test Point 31. 


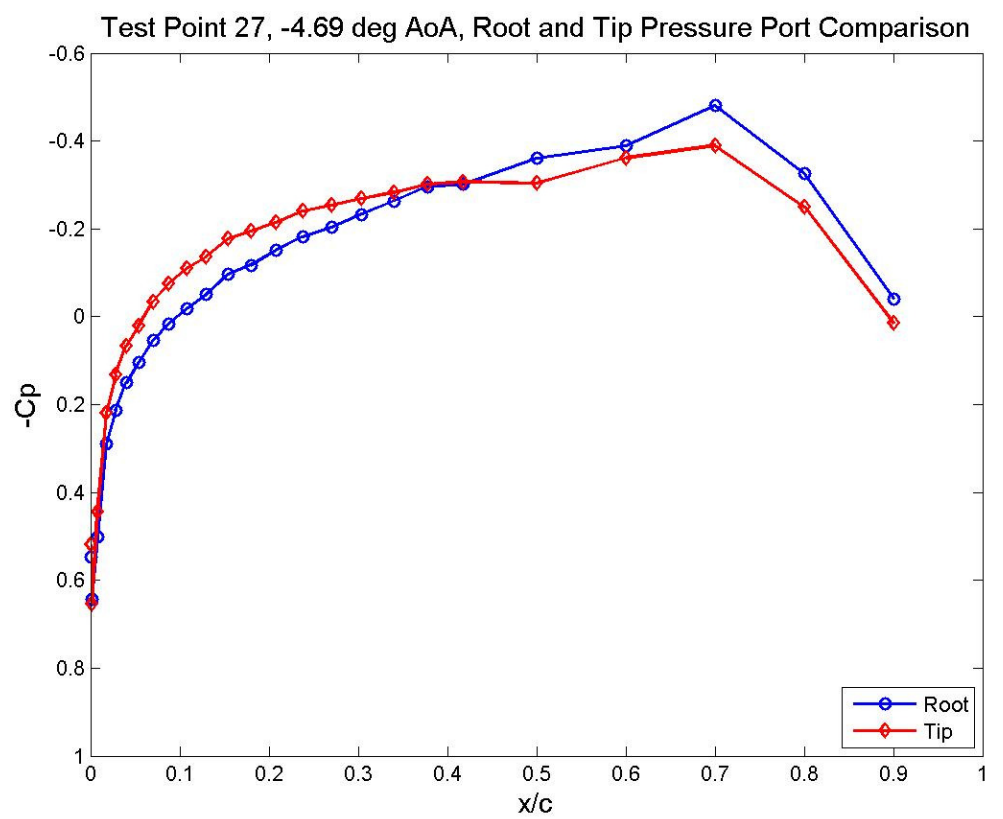

Figure 26: Root and tip pressure port comparison for Test Point 27. 


\section{BASELINE INFRARED THERMOGRAPHY}

\subsection{Infrared Thermography Overview}

Infrared (IR) thermography was used to visualize boundary-layer transition on the SWIFT model. One advantage of using this technique is that no obtrusive probes or treatments are required on the surface. When conducting boundary-layer stability experiments, the experimenter attempts to design sensors small enough to avoid disturbing the flowfield and boundary layer. With IR thermography, only a nonreflective surface and a temperature differential between the laminar region and the turbulent region are required to visualize transition. The IR camera can record a global image of transition and hence has proven to be a valuable tool for transition detection. This technique has been used in the F-15 flight experiments at NASA Dryden (Banks et al. 2000, Saric \& Reed 2002), T-34C experiments (Brandon et al. 1990), and B-737 experiments (Miley et al. 1997) with much success. It was desired to use IR thermography here to visualize the transition location for both a polished leading edge and a painted leading edge between the AoAs of -0.5 and -5.5 degrees. These AoAs spanned the entire spectrum of crossflow instability, with little crossflow growth at the upper end, and strong crossflow growth at the lower end.

\subsection{IR Camera Specifications}

A FLIR SC3000 camera was used for all IR flights. This camera operates in the 8-9 $\mu \mathrm{m}$ spectral range which is best suited for colder bodies. The SC3000 has a GaAs 
Quantum-Well Infrared Photodetector which is cooled down to $70 \mathrm{~K}$ with an onboard Stirling-motor cooler. The cooler takes approximately five minutes to cool down the sensor, so it was routine to turn on the IR camera before taxiing to the runway. The camera has a sensitivity of $0.02{ }^{\circ} \mathrm{C}$ at a temperature of $30{ }^{\circ} \mathrm{C}$, however a temperature difference of $0.3{ }^{\circ} \mathrm{C}$ between the laminar region and the turbulent region was required to clearly identify a transition pattern at the altitudes tested here. The camera has three different temperature ranges, in which the lowest range from $-20{ }^{\circ} \mathrm{C}$ to $80{ }^{\circ} \mathrm{C}$ was used for all flights. The camera is capable of high-speed sampling at $750 \mathrm{~Hz}$, but most data were sampled at $20 \mathrm{~Hz}$ as explained in Section 2.8. The SC3000 has a reported accuracy of $\pm 1{ }^{\circ} \mathrm{C}$, but to meet these specifications, the software must be adjusted for the atmospheric temperature, humidity, and model emissivity. The emissivity of the powdercoated surface on the testside of the model was fixed, but the temperature and humidity was constantly varying during the length of the dive. Consequently, the $\pm 1{ }^{\circ} \mathrm{C}$ accuracy was not matched in these experiments because an average value of both temperature and humidity was entered into the software. It was not feasible to change the settings of the camera during the dive because it would interrupt the continuous IR recording. This constraint was not limiting because most data presented here used the IR camera as a qualitative transition detection tool. One exception are the data displayed in Figures 27 and 30 where surface temperatures are pulled from the IR video data. 


\subsection{IR Thermography Procedures}

The IR camera is designed to measure the surface temperature on the model, however, this alone is not enough to observe transition. The key to visualizing boundarylayer transition with an IR camera is to force a temperature differential between the laminar region on the model and the turbulent region. An insulating material reduces heat conduction across this boundary and produces the highest contrast. The powdercoat epoxy surface on the SWIFT model, approximately $100 \mu \mathrm{m}$ thick, provides an insulating layer to reduce the surface heat conduction between the laminar and turbulent regions, thus providing a distinct transition location. At high Mach numbers the difference in laminar and turbulent adiabatic recovery temperature is enough to generate measureable temperature differentials at the surface (Banks et al. 2000). However, at low Mach numbers, such as these experiments, a convective heat transfer problem must be created to generate the needed temperature differential.

Past wind tunnel experiments have recorded quality IR images by cooling or heating the model surface. For example, in the experiments of Zuccher \& Saric (2007), liquid nitrogen was poured on the surface of models prior to starting the wind tunnel. Conversely, heating pads were placed on models to generate the same effect, but with the direction of convective heat transfer in the opposite direction. In the case of the liquid nitrogen, the warmer air heats the model through convective heating, whereas with the heating pads the air is cooling the model's surface. At low Mach numbers the temperature differential between the laminar and turbulent regions is created because the 
convective heat transfer rate is higher for a turbulent boundary layer then it is for a laminar one. If the model is colder than the freestream air, the turbulent boundary layer heats the surface at a faster rate. The laminar region heats up at a slower rate making this region appear colder. Unless heat transfer to the surface is continuous, both the laminar and turbulent regions will reach a near-constant temperature through heat conduction in the model and transition is no longer observable with the IR camera. Therefore, this technique was easy to implement in the wind tunnel and in flight, but suffers from a limited runtime capability once the model reaches close to uniform temperatures. The time in which the model reaches a uniform temperature depends on the model material, skin thickness, and difference between the freestream air temperature and the model surface temperature.

For these flight experiments, the temperature lapse rate in the atmosphere was used to generate the temperature differential between the freestream and the model surface, similar to the T-34C experiments performed at night (Brandon et al. 1990). The procedure was as follows. The model was cold soaked at 10,500 feet until a uniform temperature was reached on the model. The FTE used the IR camera to decide when the model was at a uniform temperature, which typically took 30 minutes. Later flights incorporated a 20-minute cold soak. This was also successful, but produced less transition data since these flights required more altitude before a good image was produced. As the model descended into warmer air, the turbulent region on the aft section of the model heated up faster than the laminar region. At the end of the dive it was possible to generate as much as a $3{ }^{\circ} \mathrm{C}$ differential between the laminar and turbulent 
regions, which was well within the sensitivity of the SC3000 camera. Figure 27 shows a plot of the surface temperatures in the laminar region, turbulent region, and the freestream total temperature for a flight producing good IR images at the bottom of the dive. Note that the accuracy of the surface temperatures displayed in Figure 27 is predicted to be less than $\pm 1{ }^{\circ} \mathrm{C}$ based on the argument presented at the end of Section 5.2 .

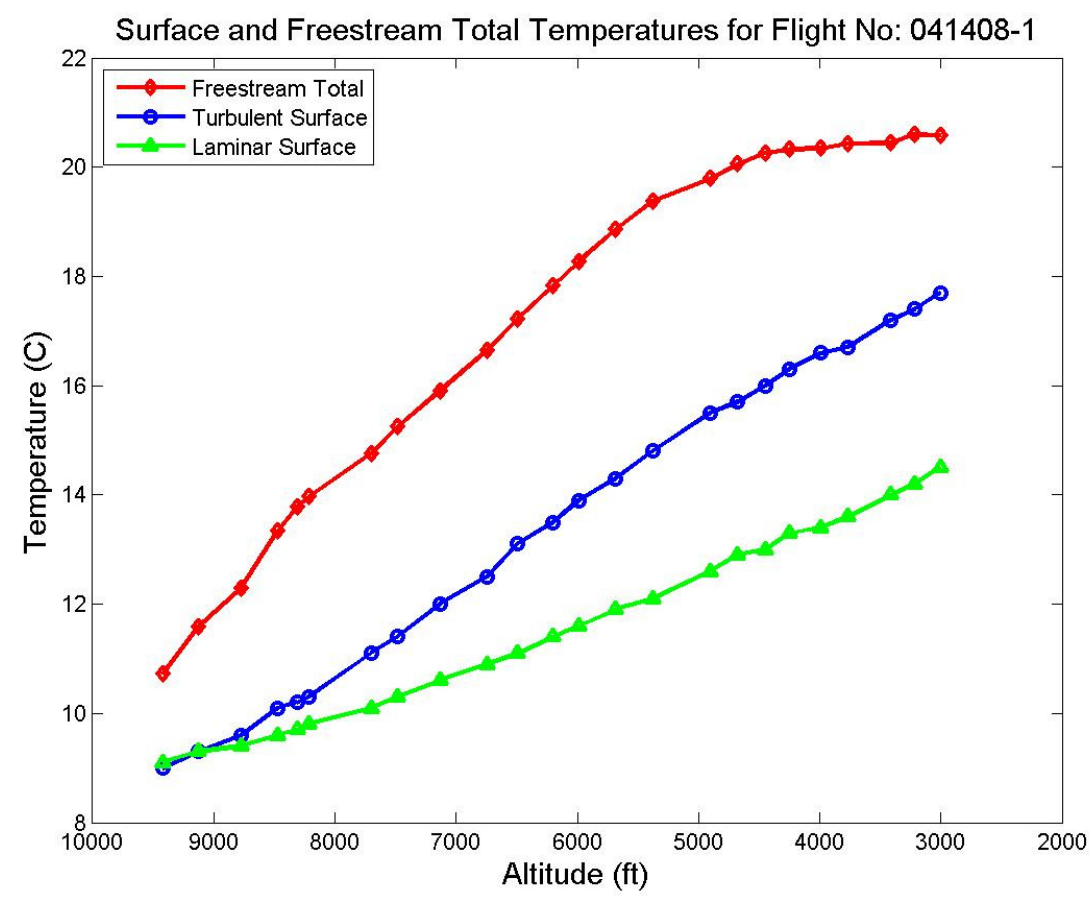

Figure 27: Plot of both laminar and turbulent surface temperatures and freestream total temperature during a dive producing good IR images.

In Figure 27 both the laminar and turbulent surface temperatures are almost equivalent and lower than the freestream total temperature at the beginning of the dive. This is indicative of a sufficient cold-soak time where the entire model surface is close to 
a uniform temperature. As the O-2A descends into warmer air, both the laminar and turbulent regions heat up, with the exception that the turbulent region heats up at a faster rate. As more altitude is lost, the temperature difference between the laminar and turbulent region increases resulting in a clearer IR image of transition with greater contrast. In Figure 27, a temperature difference of $0.3^{\circ} \mathrm{C}$ was achieved around 8500 feet. This marked the beginning of visible transition data for this dive. At the bottom of the dive, a $3{ }^{\circ} \mathrm{C}$ temperature differential was present and produced the best IR images. Furthermore, the lower altitudes also correspond to the highest $R e_{c}$ due to a decrease in kinematic viscosity. Therefore the goal was to always be on test conditions by the time the aircraft was between 5000 feet and 3000 feet to produce the best set of data. However, the lower altitudes were more prone to atmospheric turbulence. To combat this, every attempt was made to takeoff early in the morning before the atmosphere became too unstable. Furthermore, dives were never extended below 2700 feet for safety concerns. The tallest obstacle in the test area is an antenna at 1700 feet. It was desired to maintain 1000 feet of vertical clearance from this antenna.

One disadvantage of this flow-visualization technique was that the IR camera picked up radiation from hot spots on the aircraft, such as the front engine, rear engine, and especially the exhaust pipes. Furthermore, the contamination of the IR images was exacerbated by the fact that the model was mounted at an even waterline as the fuselage of the O-2A. As a result, some parts of the aircraft that were not at elevated temperatures also appeared on the IR images due to heat radiation. These superfluous features made it difficult to process the IR images initially, but most of the features were constant and 
easy to filter out once the pattern was recognized on later flights. Figure 28 shows two example IR images where all the extraneous features are clearly visible and are described in the following text.

The model has aluminum tape markers identifying the 40, 60, and $80 \% x / c$ locations. These markers are placed at the top and bottom of the model. Note that some of the early IR images presented in this dissertation only have tick marks at 40,60, and $80 \% x / c$ and do not include the numbers 40,60 , and 80 . The removable leading edge is attached at $15 \% x / c$. In Figure 28, the polished leading edge was attached for both flights represented. The highly-reflective surface resulted in very little detail that can be identified forward of $15 \% x / c$ with the IR camera. At $15 \% x / c$ there is a wavy line running the entire span of the model. This wavy line is where the powder coat on the main part of the model was hand sanded to eliminate any steps between the testside of the model and leading edge due to the material thickness of the powder coat. This feature is difficult to see in Figure 28, but appears on future IR images with the painted leading edge. At half-span, at approximately $20 \% x / c$ and $80 \% x / c$, there are hot spots indicating the fore and aft engine exhaust pipes, respectively. Running along half-span of the model is a curved line. This is the bottom of the fuselage being reflected onto the model. At the top there is a curved warm region just below the pylon. This is the reflection of the Plexiglas in the cabin, which runs parallel with the bottom of the fuselage line seen at half-span. This was confirmed in flight by opening and closing the window next to the pilot and observing a change in color with the IR camera. At the 
trailing edge, there is a small region at the remaining $5 \% x / c$ of the model that is clearly a different color with a straight line parallel to the trailing edge. This is where the model transitions to solid aluminum and is no longer hollow like the rest of the model. This difference in skin thickness shows up as a different color on all IR images. At $50 \% x / c$ there is an internal stiffener in the model. This feature shows up as a dark line on most images. There are also three chordwise internal stiffeners that show up as dark lines. Lastly, there are more identifying marks at the trailing edge. These markers are aluminum tape and identify the location for the two static pressure port rows. The root and tip pressure port row tape markers are sometimes absent in some of the IR images. This absence is because the markers are directly inline with the aft propeller and there is a blade-passing frequency seen in the IR image that causes the tape to disappear and reappear. Depending on which frame is chosen for the IR image, those tape markers are sometimes absent. 


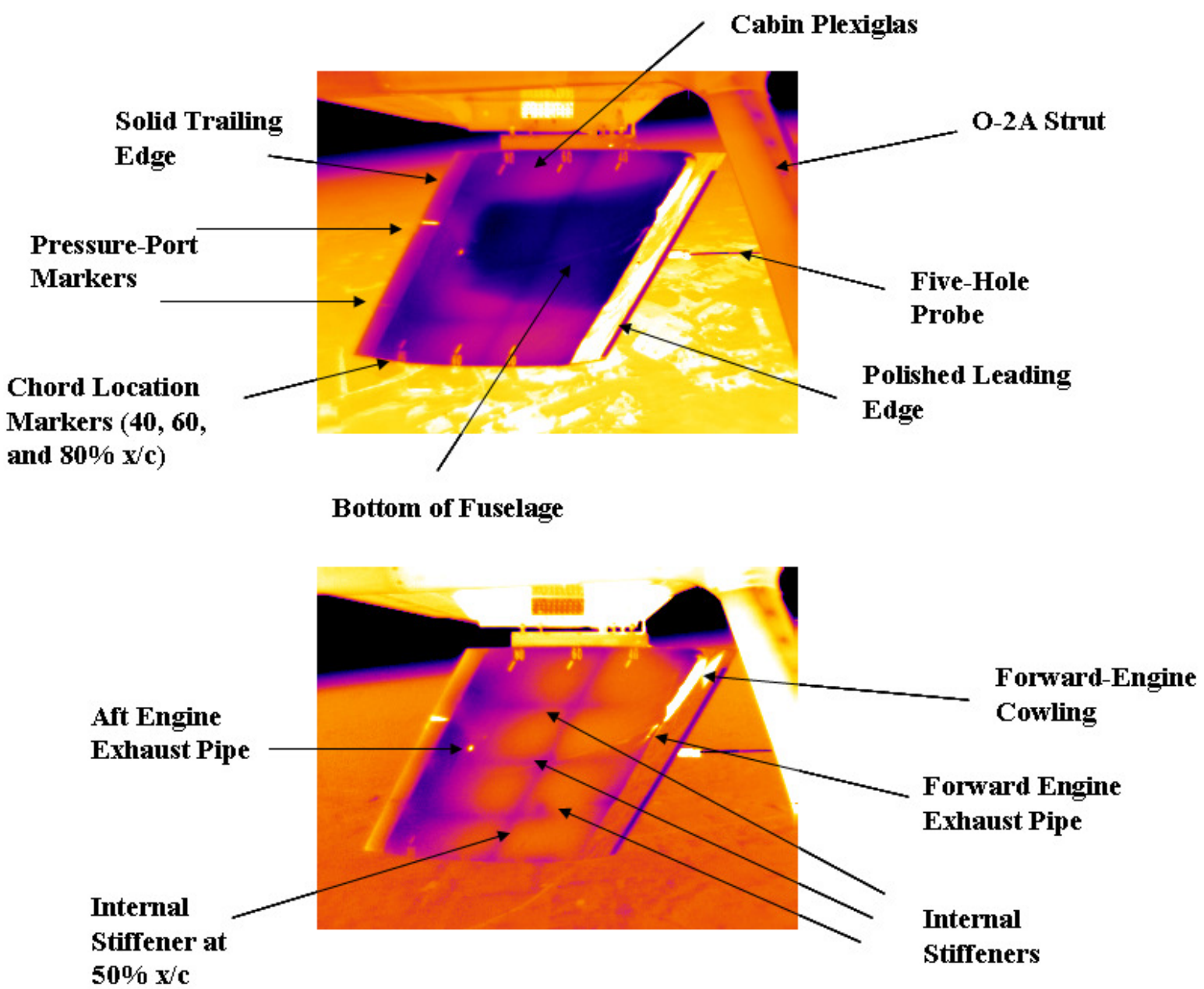

Figure 28: IR images showing radiation spots and markers on the model.

\subsection{Atmospheric Variables}

In addition to radiation contaminating the IR images, the temperature lapse rate also caused issues. On average, the lapse rate in the Troposphere is linear with a slope of approximately $-2^{\circ}$ C/1000 feet (1976 U.S. Standard Atmosphere). However, during one flight early in the program, the co-pilot observed a lapse rate contrary to those displayed in standard atmosphere tables. During the climb, the temperature increased for 1000 feet 
and then continued to decrease as more altitude was gained. This inversion in the temperature lapse rate explained some of the confusing IR images displaying diminished IR quality at the bottom of the dive. According to Figure 27, the best images should be recorded at the bottom of the dive when the temperature differential between the laminar and turbulent regions is maximized. The data in Figure 27 were collected when the freestream temperature was decreasing almost linearly with altitude, as predicted. If a temperature inversion is encountered, the air switches from heating the model, to cooling the model during the dive. When this occurs, the turbulent region starts to cool faster than the laminar region and reduces the temperature differential. When the temperature difference decreases below the empirically determined $0.3{ }^{\circ} \mathrm{C}$ threshold for the camera, transition is no longer observable. After the discovery of temperature inversions, the FTE began to continuously monitor the temperature during the climb. With this information, some of the reversed IR images recorded during the dive could be correlated, and the flight plan could be modified to make sure the model was cold soaked at the lowest possible temperature.

Early in the program, flight was limited to an altitude of 10,500 feet. However, this was later extended to 11,500 feet because temperature inversions were occasionally picked up around 9000 feet and it was beneficial to climb to a higher altitude to cold soak the model at a lower temperature. The 10,500 and 11,500 foot altitudes proved to be appropriate for cold soaking after taking into account the additional time for the normally aspirated $\mathrm{O}-2 \mathrm{~A}$ to climb to a higher altitude, FAA oxygen requirements for 
crew above 12,000 feet, and even in the coldest conditions the targeted $\operatorname{Re}_{c}$ was never reached above 6000 feet. Thus a longer dive time would only result in more data being collected below the targeted $R e_{c}$. In light of these restrictions it was not beneficial to climb above 10,500 feet unless a temperature inversion was encountered.

In southeastern Texas, weather patterns are dominated by the Gulf of Mexico, and experience with temperature inversions showed that lapse rates were best during the summer. The summer lapse rates were usually linear and rarely had temperature inversions. During the colder winter months, temperature inversions were common. Additionally, early-morning flights in the winter typically had cold surface temperatures, a large rise in temperature up to about 2000 feet, and then a gradual linear decline in temperature up to 10,500 feet. This situation was not as difficult to handle as the temperature inversions because the minimum dive altitude was 2700 feet. Therefore, the decrease in temperature at the lower altitudes was rarely reached before leveling off. Figure 29 shows three example lapse rates recorded during the course of the program. The good lapse rate recorded for Flight No: $041408-1$ was ideal because there was a large temperature difference between the top and bottom of the dive. Also, the temperature decreased linearly with altitude so the IR images were clear and predictable. The acceptable lapse rate recorded for Flight No: 091608-1 shows a smaller temperature difference between the top and bottom of the dive and also displays a large temperature inversion around 5000 feet. This lapse rate produced acceptable IR images because it was linear and good images were recorded above the inversion. At 5000 feet, the surface 
temperatures on the model started to equilibrate and the transition image disappeared with the IR camera. During flights with temperature inversions, the goal was to pass through the inversion region as quickly as possible in order to recover the IR image at the bottom of the dive. Overall this lapse rate is labeled as acceptable because the IR images were good with the exception of the data below 5000 feet. Lastly, the poor lapse rate recorded on Flight No: 063108-1 had only a few degrees of difference between the bottom and top of the dive. Furthermore, there are several inflection points in the lapse rate preventing the turbulent boundary layer to properly heat up the surface of the model for a good quality IR image. This flight produced very little useful data.

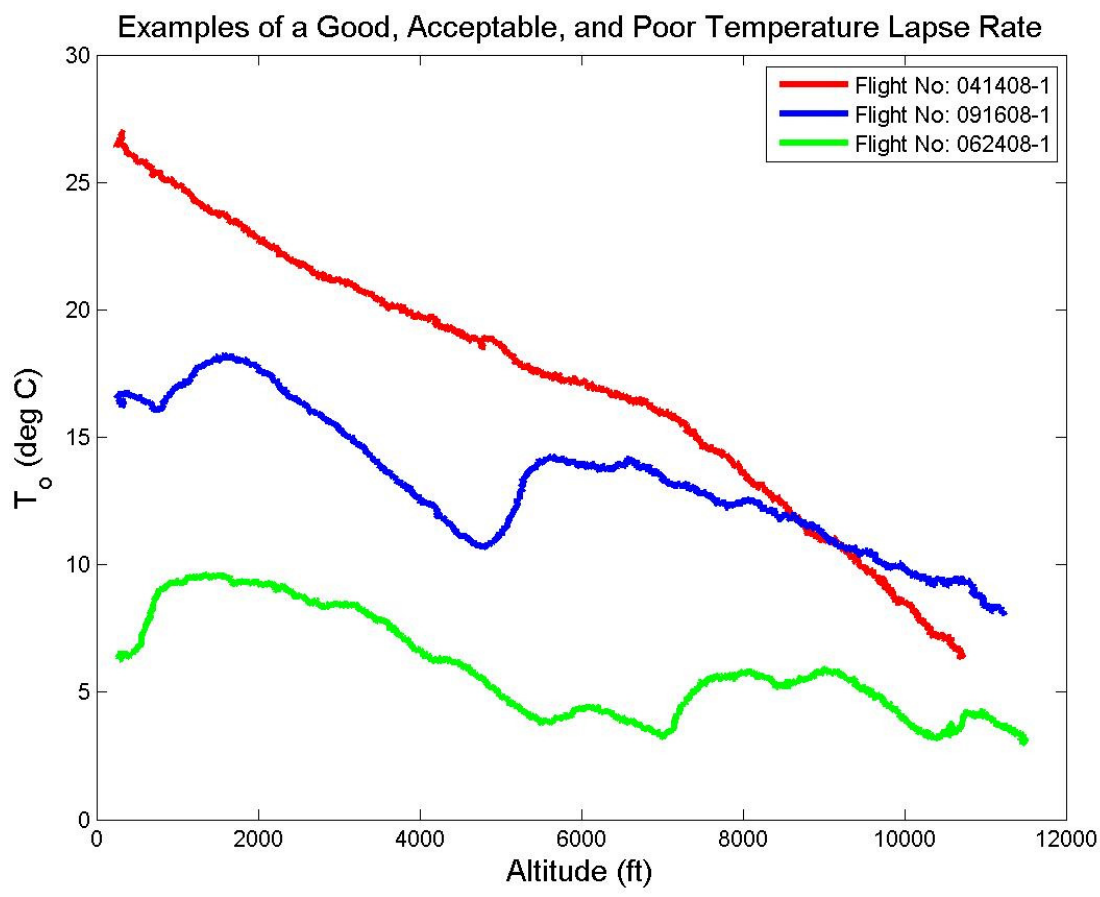

Figure 29: Total temperature lapse-rate examples recorded throughout the year. 
As a comparison to Figure 27, Figure 30 is presented for the data recorded on Flight No: 091608-1. Figure 30 shows the freestream total temperature with a large inversion near 5000 feet, in addition to the laminar and turbulent surface temperatures recorded with the IR camera. For this flight, transition data were recorded between 9000 feet and 5000 feet. At 5000 feet, the decrease in total temperature started to cool the model and the difference between the laminar and turbulent temperature decreased below the sensitivity of the IR camera. Thus the region of useful transition data stopped at 5000 feet and the transition pattern was never recovered at the bottom of the dive past the inversion. To exacerbate the problem further, temperature inversions were typically recorded around 4000 feet to 5000 feet, which were always the altitudes for picking up the targeted $R e_{c}$. As a result, some test flights only recorded transition data below the targeted $R e_{c}$ at the higher altitudes before temperature inversions were recorded at the lower altitudes. 


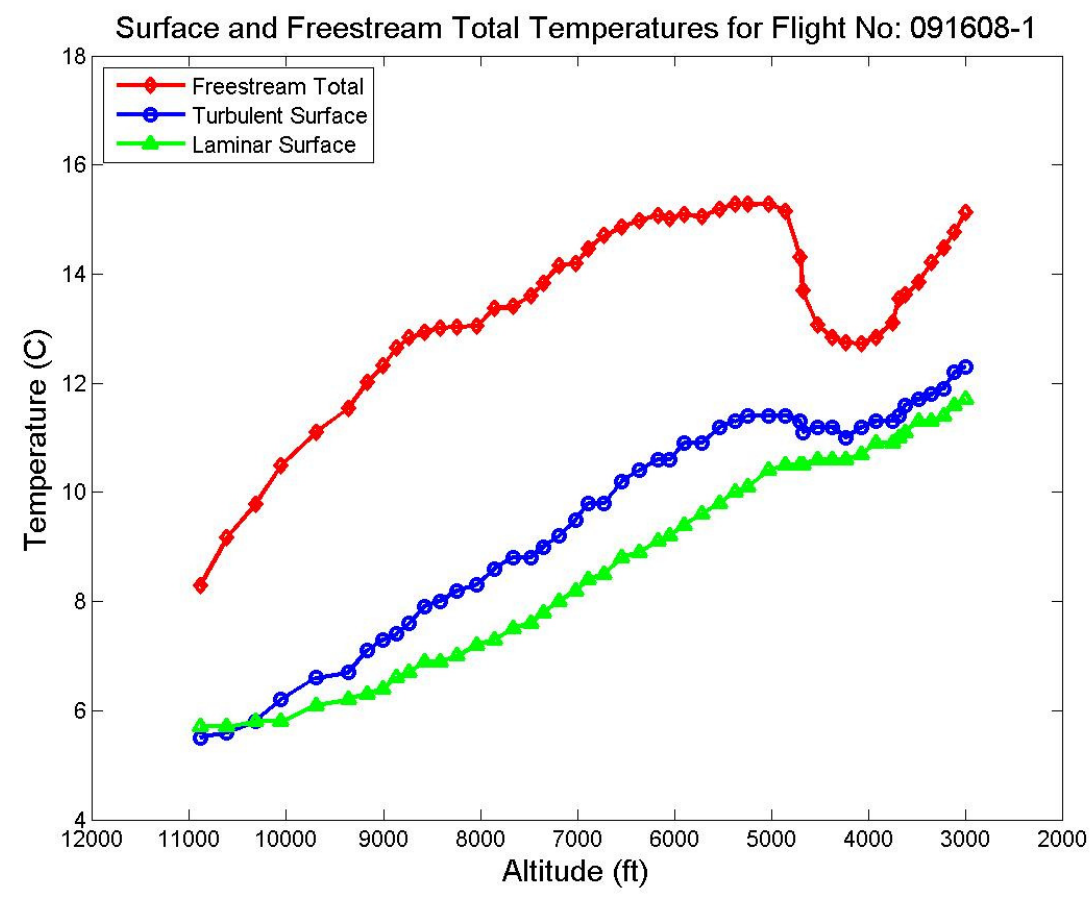

Figure 30: Plot of both laminar and turbulent surface temperatures and freestream total temperature during a dive with a temperature inversion.

Although the temperature lapse rates were typically much better in the summer, there was the added difficulty of hitting the targeted $R e_{c}$ of 7.5 million with the increase in dynamic viscosity. Moreover, there was usually more moisture coming in from the Gulf of Mexico at the lower altitudes during the summer. This made it common in the summer months to pick up condensation on the model around 3000 or 4000 feet, resulting in an instantaneous full-chord, full-span turbulent model. Whenever this occurred, the dive was terminated and a climb was initiated to try again. During the winter months, the targeted $R e_{c}$ was reached sooner and the model rarely formed condensation at the lower altitudes. This resulted in dives that could be extended down 
to 2700 feet. However, there was always the difficulty in producing good IR images as the model passed through temperature inversions, or worse, isothermal conditions. So the generation of quality IR images was not without its difficulties. Overall, the summer produced the best IR images because the lapse rates were linear and predictable. However, this gain was at the expense of taking more time to reach $R e_{c}$, in addition to the condensation difficulties experienced at the bottom of the dive. Furthermore, the summer months were prone to atmospheric turbulence which resulted in a dive termination at low altitudes. In the end, the difficulties with the IR camera were not with the operation of the camera in flight, but rather with the difficulties of generating good temperature differentials in light of the always dynamic atmospheric conditions.

\subsection{Polished Leading Edge IR Results}

Despite the difficulties with unwanted body radiation and atmospheric variables, very clear images were generated with the IR camera and dive profile setup for this program. Initially, baseline IR data were recorded with a polished leading edge and $A o A$ values between \pm 2 degrees, as measured with the air data boom on the starboard wing. These $A o A$ values have been corrected based on the findings explained in Section 2.9. With the polished leading edge, baseline IR images were recorded from corrected AoAs between -0.65 and -4.39 degrees. Figures 31 through 34 show four example IR images with the polished leading edge as the $A o A$ is decreased. Note that the five-hole probe is not present in these images because it was added after these images were taken. In each of these figures, the turbulent regions are at the aft section of the model and are depicted 
with a warmer temperature. The laminar region is depicted with a colder temperature and is always upstream of the turbulent region. The reader is reminded that in all images, the flow direction is from right to left. Using Figure 31 as an example, transition is at $80 \% x / c$ at half-span and above. The lower half of the model shows transition moving forward along a line intersecting $80 \% x / c$ at half span to $40 \% x / c$ at the wingtip. As the $A o A$ is decreased, the crossflow instability is destabilized and transition moves forward. Using Figure 34, transition has moved forward to $50 \% x / c$ at half-span at an $A o A$ of 4.39 degrees. The characteristic saw-tooth pattern, described in Dagenhart et al. 1989, is also present in this image, indicating a crossflow-dominated transition for this test point.

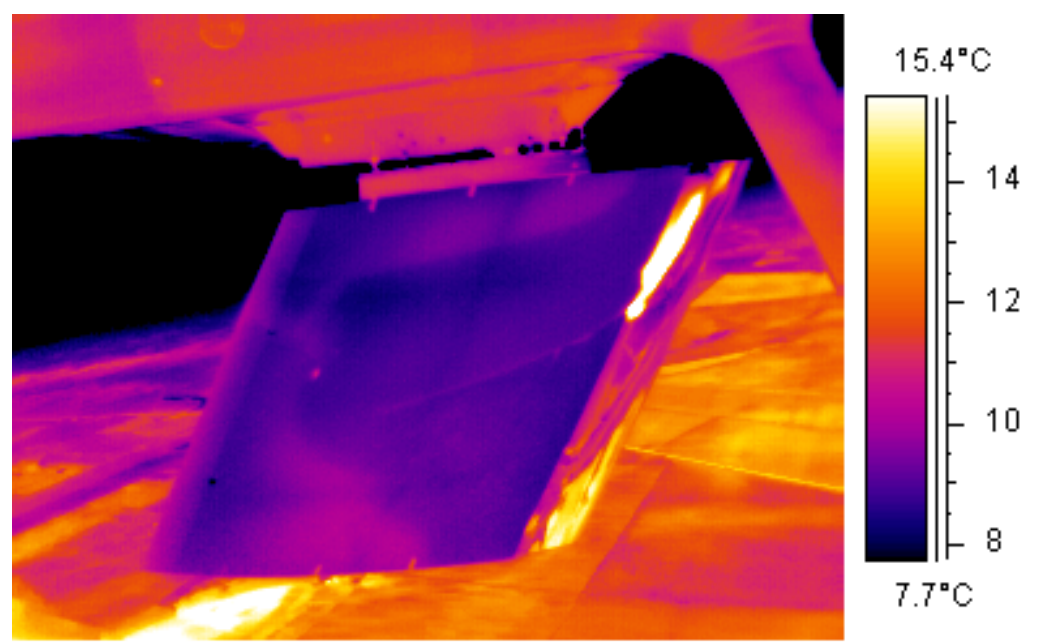
$\mathrm{AoA}=-0.65 \mathrm{deg}$
Flight No: 032106-2 $\mathrm{Re}_{\mathrm{c}}=7.45$ million
Time: 13:07:27

Figure 31: Baseline IR image with the polished leading edge at -0.65 degrees AoA. 


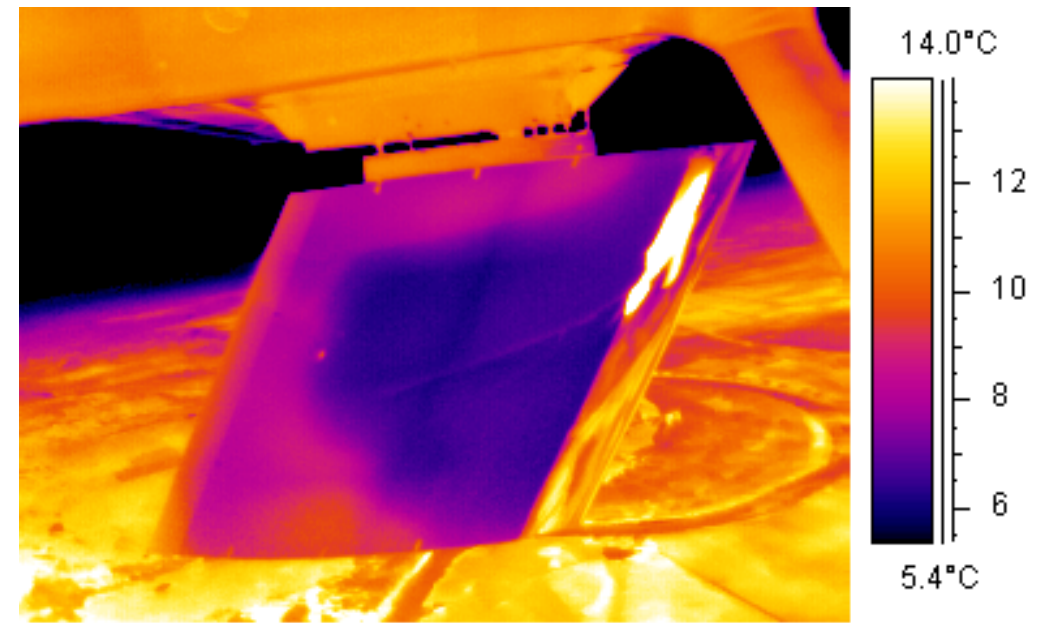

$$
\begin{array}{ll}
\text { AoA }=-1.88 \text { deg } & \text { Flight } N_{0}: 032106-2 \\
\operatorname{Re}_{c}=7.46 \text { million } & \text { Time: } 12: 55: 28
\end{array}
$$

Figure 32: Baseline IR image with the polished leading edge at -1.88 degrees AoA.

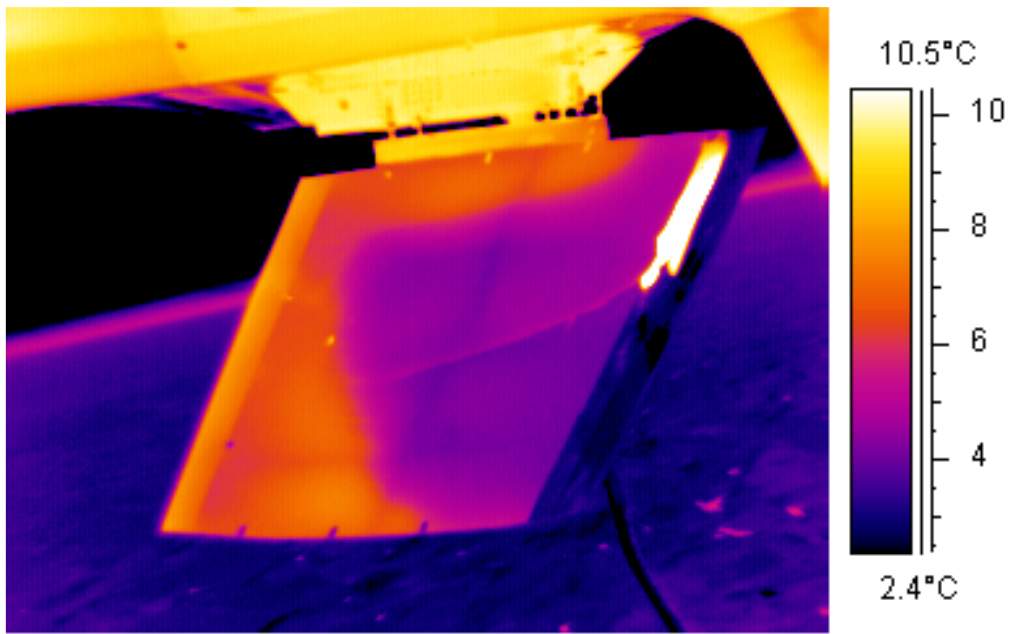

$$
\begin{array}{ll}
\mathrm{AoA}=-3.17 \text { deg } & \text { Flight } \mathrm{N}_{0}: 032206-1 \\
\operatorname{Re}_{c}=7.42 \text { million } & \text { Time: 07:31:13 }
\end{array}
$$

Figure 33: Baseline IR image with the polished leading edge at -3.17 degrees AoA. 


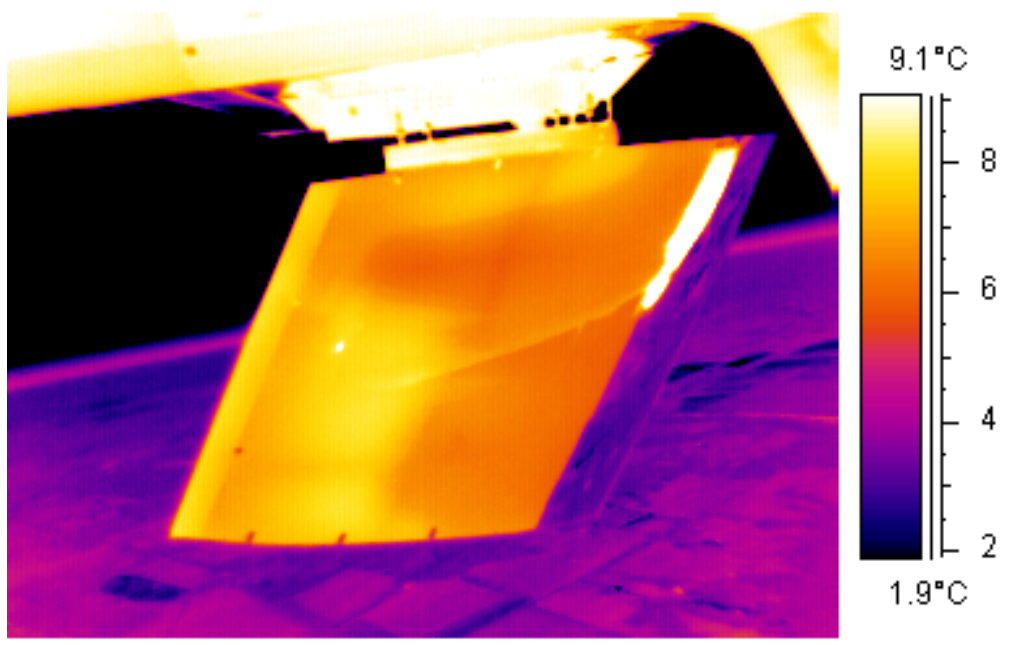

$$
\begin{array}{ll}
\mathrm{AoA}=-4.39 \mathrm{deg} & \text { Flight } \mathrm{N}_{0}: 032206-1 \\
\mathrm{Re}_{\mathrm{c}}=7.16 \text { million } & \text { Time: } 07: 53: 06
\end{array}
$$

Figure 34: Baseline IR image with the polished leading edge at -4.39 degrees AoA.

Considering Figure 31, there are a few factors that might be affecting the early transition pattern at the bottom half of the model. Primarily the laminar boundary layer below the tip pressure port row might be destabilized by a wingtip vortex. It is also possible that the four-degree cut at the bottom of the model is affecting the transition pattern. The $C p$ measurements presented in Section 4 displayed a spanwise variation on the model. The transition pattern follows this trend with the top region of the model appearing to be more stable than the bottom half of the model. In all four figures, the reflection of the cabin Plexiglas explained in Section 5.3 appears to be a turbulent region based on the color of the IR image. The reader is reminded that this is the reflection of the cabin Plexiglas and is constant for all IR images presented in this dissertation. With the exception of the laminar region in the upper right hand section of the model, the top six inches cannot be used for detecting transition due to this Plexiglas reflection. 
Therefore, it is assumed that any laminar flow observed just below the Plexiglas reflection area is extended to the top of the model. Lastly, using Figure 34 at -4.39 degrees $A o A$, despite showing early transition at the center of the model, there is still a laminar region at the top of the model extending back to $80 \% x / c$. This region is constant in many IR images and appears to be independent of surface roughness at the leading edge and all AoAs above -5 degrees. The laminar boundary layer above the root pressure port row might be stabilized by the influence of the O-2A wing above this region. It is difficult to accurately determine what is going on in this region because the pressure ports do not extend that high, and as result, no stability calculations can be performed there based on experimental $C p$ data. This stable region of laminar flow reinforces the assumption that the Plexiglas region at the top of the model is also laminar for it is even closer to the O-2A wing.

With a polished leading edge, NLF was achieved beyond the pressure minimum at all test points with AoAs greater than -3 degrees. This surprised the FRL team because the model was predicted to transition to turbulence around $50 \% x / c$ at zero degrees AoA, not -4 degrees. Because transition was observed beyond the pressure minimum, the instability for this case was probably T-S waves due to the adverse pressure gradient aft of $70 \% x / c$. Furthermore, transition has never been observed beyond the $80 \% x / c$ location regardless of leading edge roughness or stabilizing $A o A$ condition. $80 \%$ laminar flow is the most laminar flow observed during these experiments. The large amounts of NLF on this model confirmed the belief that the flight environment is indeed a lowdisturbance environment. It also confirms the fact that stability experiments must be 
performed in low-disturbance wind tunnels or in flight. If not, transition predictions made in a noisy wind tunnel are not valid for flight.

Even though all baseline IR images were recorded at incorrect $A o A$ values with the starboard-mounted air data boom, the entire baseline IR campaign provided experience and confidence with identifying transition. It also provided assurance in predicting the transition location for future tests. A unique characteristic of this airfoil occurs when the model has large amounts of NLF. When transition is not crossflow dominated, laminar flow is recorded past the pressure minimum at $70 \% x / c$ and is observed at $80 \% x / c$. Once this much laminar flow is achieved, any increase in $A o A$ stabilizes the crossflow and transition remains at $80 \% x / c$. This feature provided confidence later in the program, where regardless of leading edge configuration or roughness, an increase in $A o A$ was always stabilizing producing more laminar flow up to the observed maximum of $80 \% x / c$. Furthermore, once $80 \%$ laminar flow was achieved there was no point in increasing the $A o A$ further. On the other hand, a decrease in $A o A$ was always destabilizing and transition would eventually move forward from the $80 \%$ $x / c$ location due to the excited crossflow instability.

The subcritical DREs used for LFC were never designed to work under these conditions with laminar flow beyond the pressure minimum. Control DREs prevent growth of the most unstable crossflow wavelength in order to increase the amount of laminar flow on the swept-wing. With very little crossflow growth, the control DREs 
would do nothing. With the polished leading edge, only Figure 34 shows signs of a crossflow-dominated transition. Only in Figure 34 at -4.39 degrees $A o A$ is there a condition where the DREs could help extend the amount of laminar flow. As a result, it was decided to excite the crossflow instability in order to move transition forward. Once this occurred, the effectiveness of the control DREs would be demonstrated by pushing transition back to the original, polished baseline location.

\subsection{Crossflow Excitation}

There are several ways of moving transition forward on the swept-wing. Crossflow vortices can be destabilized by decreasing the $A o A$, increasing the $R e_{c}$, increasing the $T u$, increasing $\Lambda_{L E}$, and/or increasing the 3-D roughness near the leading edge. It was desired to demonstrate the DREs at an $A o A$ close to the designed $A o A$ conditions for SensorCraft, which are \pm 2 degrees. As a result, decreasing the AoA lower than -4.39 degrees was not preferred. The FRL tried increasing the $R e_{c}$ by deploying to a colder base of operations. One week of flight operations were conducted in Coffeyville, KS, with the polished leading edge. There, a $\operatorname{Re}_{c}$ of 8 million was achieved but transition was still observed at $80 \% x / c$ with the polished leading edge. The third option of adjusting the freestream turbulence was not a viable option. This option might be available for a wind tunnel experiment, but not possible for these flight experiments. $\Lambda_{L E}$ could not be increased because it was fixed at 30 degrees per the requirements for SensorCraft. Lastly, the FRL tried to increase the 3-D roughness close to the attachment 
line by painting the leading edge. Besides changing the $A o A$, painting the leading edge was the easiest to perform of the five options listed here.

Before painting the leading edge, the surface roughness of the polished leading edge was measured to record the conditions of the experiment. Using the procedure outlined in Section 2.11.1, the surface roughness of the polished leading edge was measured to be $0.33 \mu \mathrm{m}$ rms with $2.20 \mu \mathrm{m}$ average peak-to-peak values.

\subsection{Painted Leading Edge IR Results}

The leading edge was painted in order to excite the crossflow instability and move transition forward. This would eliminate the need for a highly negative $A o A$ and also eliminate the need to operate above the targeted $R e_{c}$ of 7.5 million. The first attempt to move transition forward with a painted leading edge failed. The polished aluminum was covered with an epoxy primer and Jet Glow polyurethane paint thinned to a ratio of 4:1. Past wind tunnel experiments proved that a paint job of moderate quality was enough to excite the crossflow instability. However, in these flight experiments the first round of paint failed to move transition forward. Unfortunately the surface roughness for this condition was never measured. A second coat of paint was applied to the leading edge, only this time a thinning agent was not added to the paint. This was purposely done to produce an "orange peel" effect. This technique was more successful because early transition was recorded at moderately negative AoAs. IR images with this painted leading edge are in Section 8 with the control DREs. The painted IR results presented in 
this section were taken with the $C p$ leading edge. Late in the program, the $C p$ leading edge was needed to demonstrate LFC with the DREs. As a result, the pressure ports were filled with Bondo to eliminate the wedges discovered above 7.2 million $\operatorname{Re}_{c}$. The $C p$ leading edge was primed with an epoxy primer and Jet Glow polyurethane paint with no thinning agent. The color of choice was white in order to enhance the contrast between the black DREs and the leading edge. Figures 35 through 38 shows IR images of four different $A o A$ conditions with this painted leading edge. Note that the leading edge now has a flat finish to it so detail in this region can be captured with the IR camera. The area where the powder-coated testside was sanded to mate up with the leading edge is also clear in the IR images depicted below. This region is absent of paint and shows up as a reflective surface because it is bare aluminum. Another addition in Figures 35 through 38 is the five-hole probe extending forward of the leading edge. The images depicted in these four figures were recorded after adding the five-hole probe, so the $A o A$ values do not require a correction. 


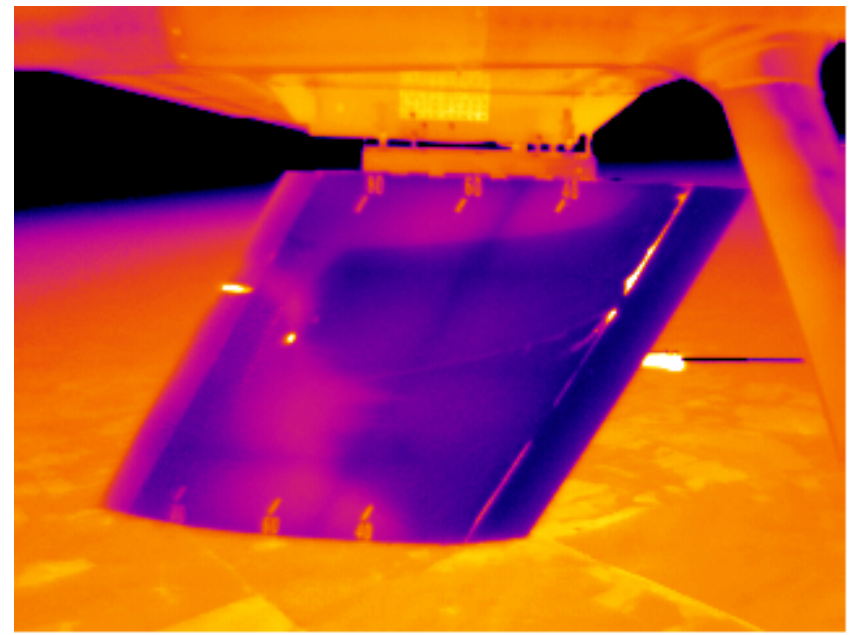

$18.4^{\circ} \mathrm{C}$

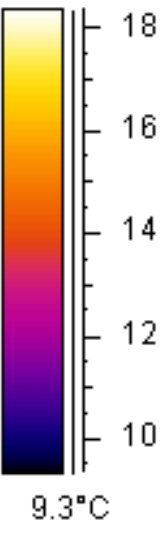

$$
\begin{array}{ll}
\mathrm{AoA}=-3.55 \mathrm{deg} & \text { Flight No: 090408-1 } \\
\operatorname{Re}_{\mathrm{c}}=7.22 \text { million } & \text { Time: 09:25:25 }
\end{array}
$$

Figure 35: Baseline IR image with the painted leading edge at -3.55 degrees AoA.
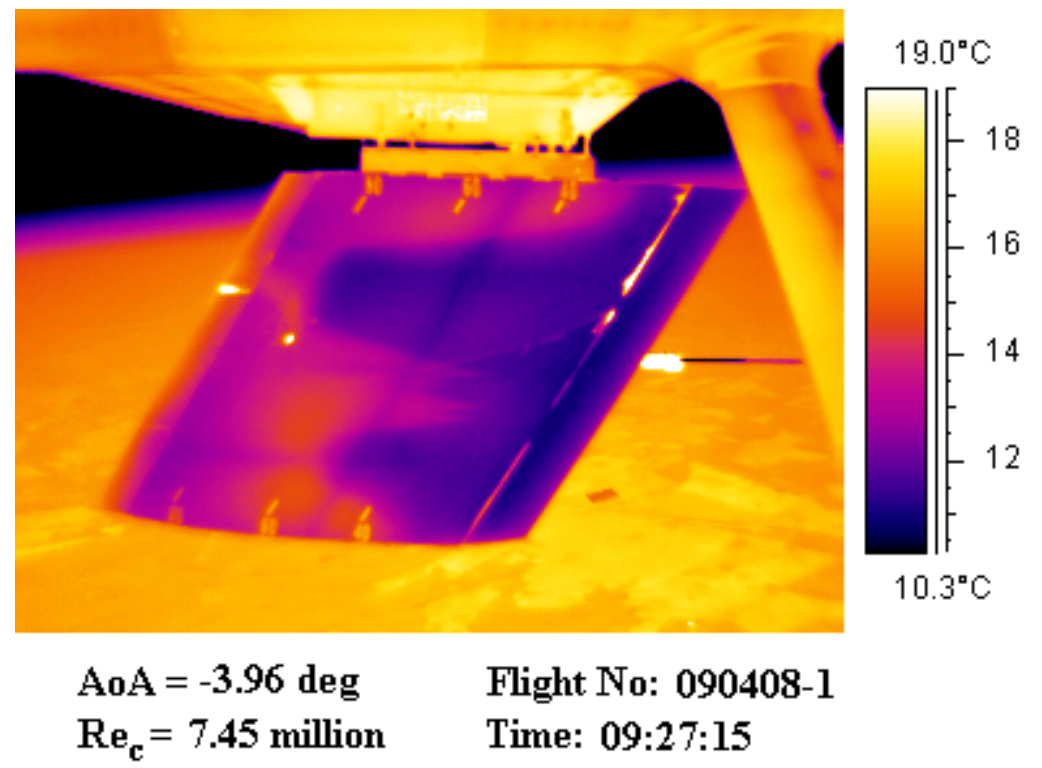

Figure 36: Baseline IR image with the painted leading edge at -3.96 degrees AoA. 


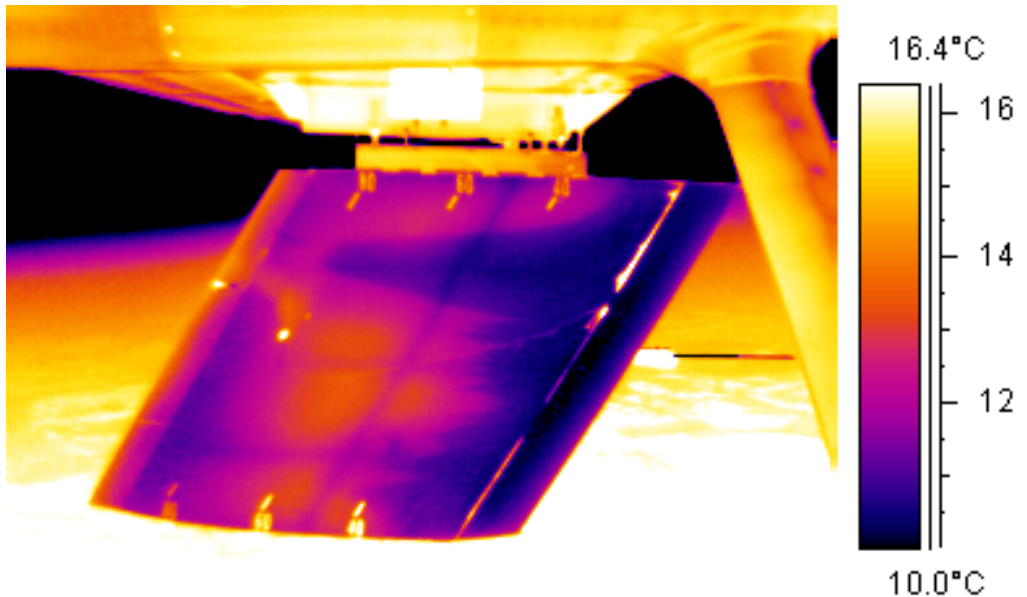

$$
\begin{array}{ll}
\text { AoA }=-4.61 \text { deg } & \text { Flight No: 090408-1 } \\
\operatorname{Re}_{c}=7.48 \text { million } & \text { Time: 10:08:26 }
\end{array}
$$

Figure 37: Baseline IR image with the painted leading edge at -4.61 degrees $A o A$.
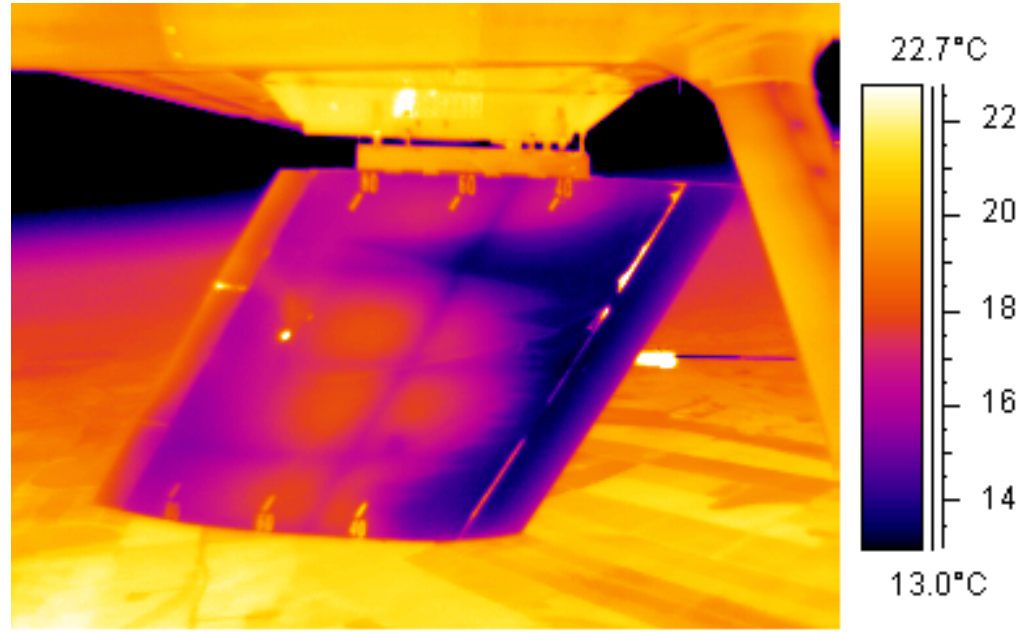

$$
\begin{array}{ll}
\text { AoA }=-5.07 \text { deg } & \text { Flight No: 090408-1 } \\
\text { Re }_{c}=7.40 \text { million } & \text { Time: 10:08:50 }
\end{array}
$$

Figure 38: Baseline IR image with the painted leading edge at -5.07 degrees AoA. 
Figures 35 through 38 show IR images with the painted leading edge at $A o A$ between -3.55 degrees and -5.07 degrees. At -3 degrees $A o A$ the model is laminar back to $80 \% x / c$, much like the IR image depicted in Figure 31 with the polished leading edge. However, there is a big jump in transition location in Figure 36 when the $A o A$ is decreased to -3.96 degrees. This large jump in transition location is due to the elevated crossflow instability with the painted leading edge. A decrease in AoA of less than half a degree moves transition forward from $50 \% x / c$ to $30 \% x / c$, although only a small section in the center of the model moves forward. As the $A o A$ continues to decrease, there is not a large forward jump in the transition location as in Figure 36, but a more gradual growth in spanwise turbulence. At -5.07 degrees $A o A$, the entire model starts to fill in with a turbulent transition front that moves forward slightly to approximately $20 \% x / c$. Another observation with the images in Figures 35 through 38 is that the painted leading edge did very little to destabilize the regions above and below the root and tip pressure port rows, respectively. It is not until -5.07 degrees $A o A$ that these two regions start to fill in and produce a uniform transition front at $20 \% x / c$.

As in the polished leading edge case, the surface roughness of the painted leading edge was measured. The first $2 \% x / c$ was sanded down with 1000 -grit sand paper to provide a uniform base for future DRE flights. This section had an rms roughness of $1.66 \mu \mathrm{m}$, and average peak-to-peak values of $7.69 \mu \mathrm{m}$. The region from $2 \% x / c$ to $15 \%$ $x / c$ was untouched after painting. This section had a rms surface roughness of $6.24 \mu \mathrm{m}$, with average peak-to-peak values of $31.42 \mu \mathrm{m}$. 


\subsection{Baseline IR Comparison}

A comparison between the polished and painted leading-edge transition data can be made. The AoAs tested during the painted leading-edge campaign were more negative than the polished leading edge test points. This is contradictory to the goal of studying $A o A$ values near \pm 2 degrees per the design requirements for SensorCraft. However, it was discovered that a combination of both a painted leading edge and a negative $A o A$ below -2 degrees was required to generate strong crossflow growth on this model. This argument is based on an operating $R e_{c}$ of 7.5 million. More deployments to colder temperatures and operation at $R e_{c}=8$ million might reduce the need to go to large negative $A o A$ conditions.

Figure 39 shows a plot of transition Reynolds number, $R e_{\text {trans }}$, as a function of $A o A$ for both the polished and painted leading edge. The polished leading-edge transition data show a flat line for $R e_{\text {trans }}$ on the right hand side of the figure. At $A o A$ values greater than -2 degrees, laminar flow is never extended beyond $80 \% x / c$. This results in a maximum $R e_{\text {trans }}$ of $6.0 \times 10^{6}$. As the $A o A$ decreases, the $R e_{\text {trans }}$ also decreases linearly for the polished leading edge. For the painted leading edge, a similar trend is observed at AoA values above -3.5 degrees. Under these conditions it appears as though leading edge surface roughness has little effect, and the dominating instability is a combination of crossflow and T-S waves. However, below an $A o A$ of -3.5 degrees, leading-edge roughness has a significant role in transition and the dominating instability is crossflow. Below -3.5 degrees $A o A$ there is a significant drop in $R e_{\text {trans }}$ for the painted leading edge 
data that are not observed with the polished leading edge. Figure 39 agrees with the Radeztsky et al. (1993a) experiments demonstrating that boundary layers with strong crossflow vortices are highly sensitive to leading edge roughness.

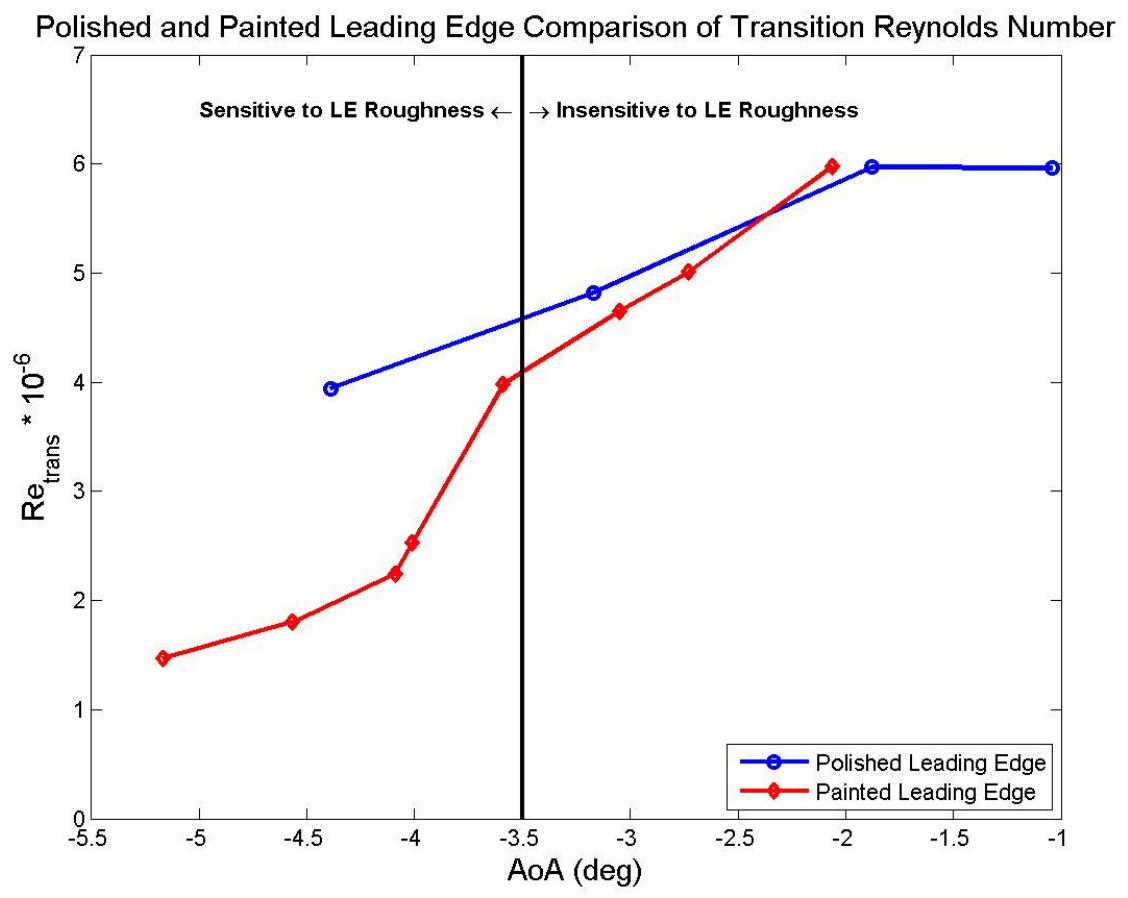

Figure 39: Plot of transition Reynolds number for various AoAs and leading-edge roughness conditions. 


\section{CROSSFLOW EXCITATION WITH CRITICAL DRES}

\subsection{Literature Review}

In a crossflow dominated boundary layer, transition occurs due to the growth of the most unstable crossflow wavelength, $\lambda_{\text {crit }}$ LST is used for determining $\lambda_{\text {crit }}$ and the neutral point, or location where $\lambda_{\text {crit }}$ initially grows. Before the neutral point, disturbances decay. Aft of the neutral point, crossflow vortices become unstable. If one excites, i.e. (promotes the growth of $\lambda_{\text {crit }}$ ), then early transition occurs. On a 45-degree swept wing, Radeztsky et al. (1993a) showed that within the laminar region downstream of an isolated, cylindrical roughness element, $\lambda_{\text {crit }}$ was excited due to the presence of the roughness element. A turbulent wedge formed downstream of the isolated roughness element. As the height of the roughness was increased in increments of $6 \mu \mathrm{m}$, the turbulent wedge moved closer to the roughness element. The diameter was also modulated to determine the most effective diameter, which was on the order of $40 \%$ to $50 \%$ of $\lambda_{\text {crit }}$ for exciting the crossflow instability. No effect was observed for roughness elements with diameters smaller than $10 \%$ of $\lambda_{\text {crit }}$. Furthermore, Radeztsky et al. (1993a) showed the neutral point for $\lambda_{\text {crit }}$ was the most effective location for locating isolated roughness. The disturbance generated by a roughness element placed forward of the neutral point will decay, reducing its effectiveness. On the other hand, aft of the neutral point, the roughness height compared to the boundary-layer height is too small to be effective. 
Reibert et al. (1996) then took a spanwise-periodic array of cylindrical roughness elements with a spacing equal to $\lambda_{\text {crit }}$ and applied them at the neutral point of the same swept wing used in the Radeztsky et al. (1993a) experiments. For this case, the DREs forced a uniform transition front that was spanwise invariant. Unlike natural transition due to random surface roughness at the leading edge, the transition front generated by critically spaced DREs was uniform. This transition pattern was then used to understand crossflow amplitude growth on the swept wing, as it provided an idealized set of spanwise invariant boundary conditions. Stationary crossflow amplitudes were measured by performing boundary-layer scans with a hotwire. An example of these hotwire scans is provided in Figure 3. In this manner, Reibert et al. (1996) showed that the crossflow vortex amplitude grows exponentially, but then demonstrates nonlinear amplitude saturation. It was also shown that the saturation level was independent of the initial roughness height.

In the SWIFT experiments, it was desired to also use critical DREs to excite early transition with the polished leading edge. The reasons for this were twofold. First the critical DREs could be used to confirm the stability calculations. According to LST, early transition should ensue if $\lambda_{\text {crit }}$ is excited. Secondly, the critical DREs could be used for making receptivity measurements to better understand the importance of roughness height. More detail on the receptivity measurements is provided in Section 7. 


\subsection{Stability Calculations}

After completing $C p$ measurements and demonstrating good agreement with computational results, stability calculations were performed for calculating neutral points, $\lambda_{\text {crit }}$, and ultimately a scheme for performing SWLFC. The two AoAs presented in Section 4 were targeted for performing stability calculations. This section focuses on the -2.61 degree $A o A$ condition to try and excite early transition with the use of criticallyspaced DREs. Section 7 deals with receptivity measurements at both AoAs of interest, and lastly Section 8 explains the SWLFC scheme for preventing growth of $\lambda_{\text {crit }}$. That section deals mainly with the -4.69 degree $A o A$ condition in order to delay transition with subcritically-spaced DREs.

\subsubsection{Linear Stability Theory}

$N$-factors can be calculated for the two test points of interest. Recall the following relationship for $N$ :

$$
N=\ln \left(A / A_{0}\right)=\int_{x_{0}}^{x}-\alpha_{i} d x
$$

where $A_{0}$ is the amplitude at the first neutral stability point and $A$ is the amplitude at $x$. Therefore an $N$-factor of 9 represents disturbance amplitude growth equal to $\mathrm{e}^{9}$. The value of $N$ at transition is uncertain, but in general an $N$-factor greater than 9 is expected for flight experiments. Figure 40 shows $N$-factors for Test Point 31 at -2.61 degrees AoA 
along the root pressure port row. When calculating $N$-factors different spanwise wavenumbers, $\beta_{t}$, are isolated to compare their growth rates. Because stationary crossflow vortices are expected to dominate the transition process, only stationary, i.e. $\omega$ $=0$, vortices are considered. In Figure 40 the $5 \mathrm{~mm}$ and $4.5 \mathrm{~mm}$ spaced crossflow vortices demonstrate the highest growth. At $70 \% x / c$ an $N$-factor of 10 is achieved. The reader is reminded that this $A o A$ demonstrated laminar flow back to $80 \% x / c$ at an even larger $R e_{c}$ than the ones calculated here. Thus $N$-factors are expected to be even higher at the targeted test conditions. Note again that the spacing of $4.5 \mathrm{~mm}$ is measured parallel to the leading edge.

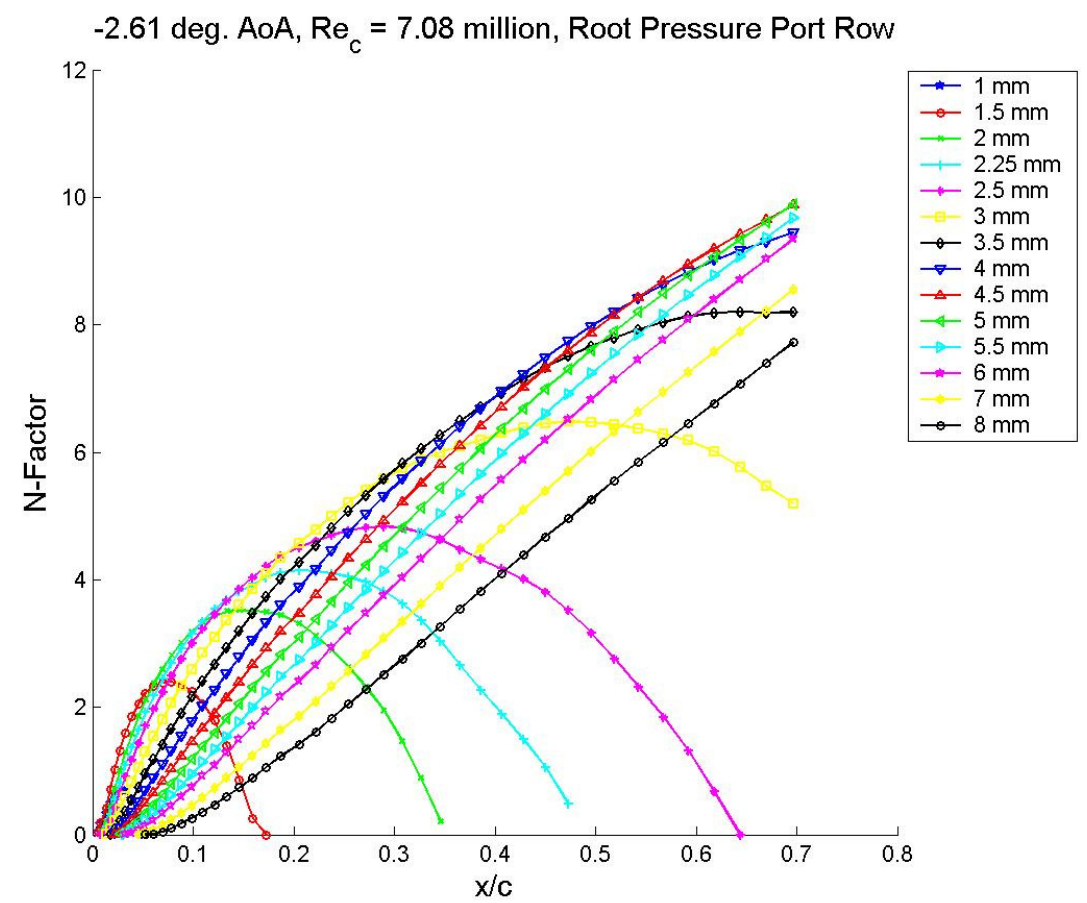

Figure 40: $N$-factors for Test Point 31 at -2.61 degrees $A o A$ along the root pressure port row. 
Figure 41 shows the $N$-factors for the same condition at the tip pressure port row. Like the root, the $5 \mathrm{~mm}$ and $4.5 \mathrm{~mm}$ crossflow vortices are the most unstable. However, the $N$-factors calculated for the tip are much lower at a maximum of 6 . This is contrary to the baseline IR images presented in Section 4 where the top half of the model demonstrated more laminar flow. This paradox supports the hypothesis that the early transition location at the tip pressure port row is probably caused by a tip vortex, or an end effect with the 4-degree cut at the bottom, and not a result of increased crossflow growth there.

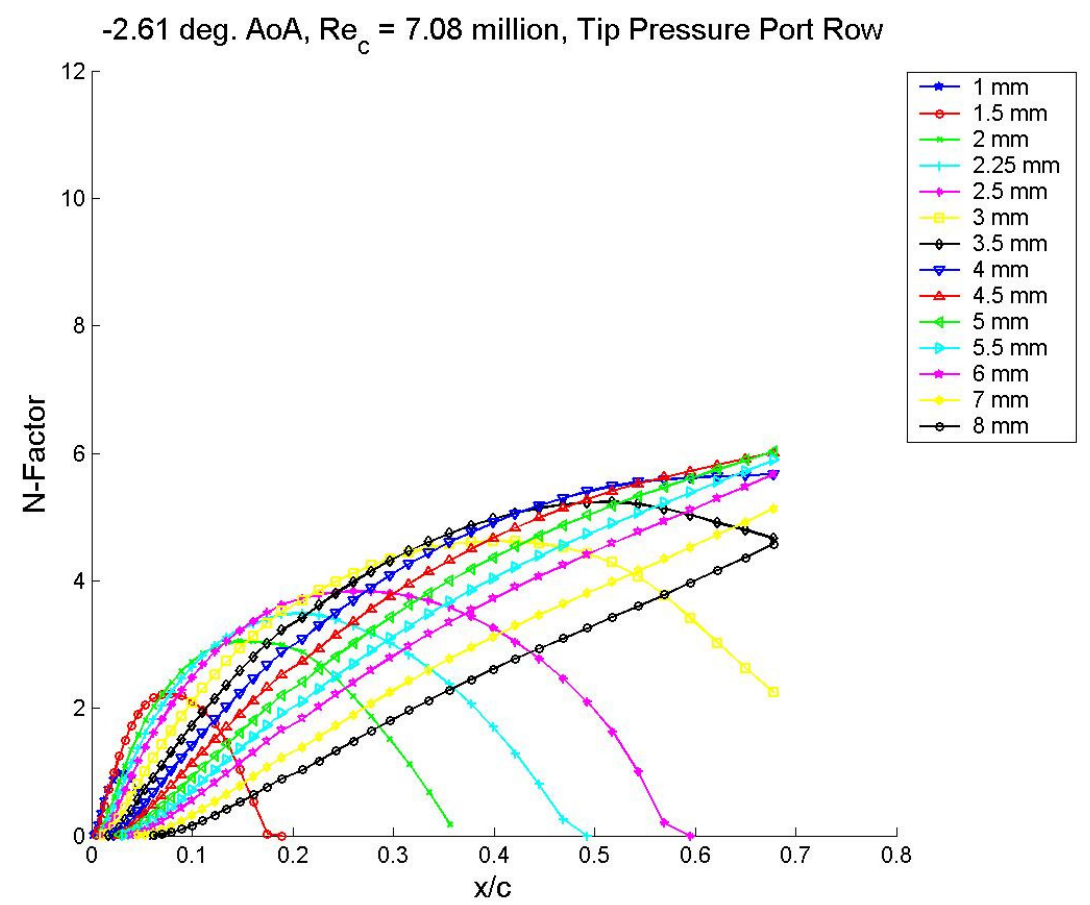

Figure 41: $N$-factors for Test Point 31 at -2.61 degrees $A o A$ along the tip pressure port row. 
Figure 42 shows the $N$-factors calculated for Test Point 27 at -4.69 degrees $A o A$. As expected, this test point demonstrates higher $N$-factors than the more stable AoA at 2.61 degrees. For this $A o A$ transition is estimated to be at $60 \% x / c$ for both the root and tip pressure port row. Figure 42 shows that this also corresponds to an $N$-factor of 10 at transition. Like Test Point 31, the tip demonstrates lower $N$-factors despite showing an equivalent transition location for both the root and tip pressure port rows. Again this discrepancy is believed to be generated by a possible end effect at the wingtip of the model. Figure 43 shows the same trend calculated for the tip pressure port row at this less stable $A o A$. This figure also shows lower $N$-factors at the tip as did Test Point 31.

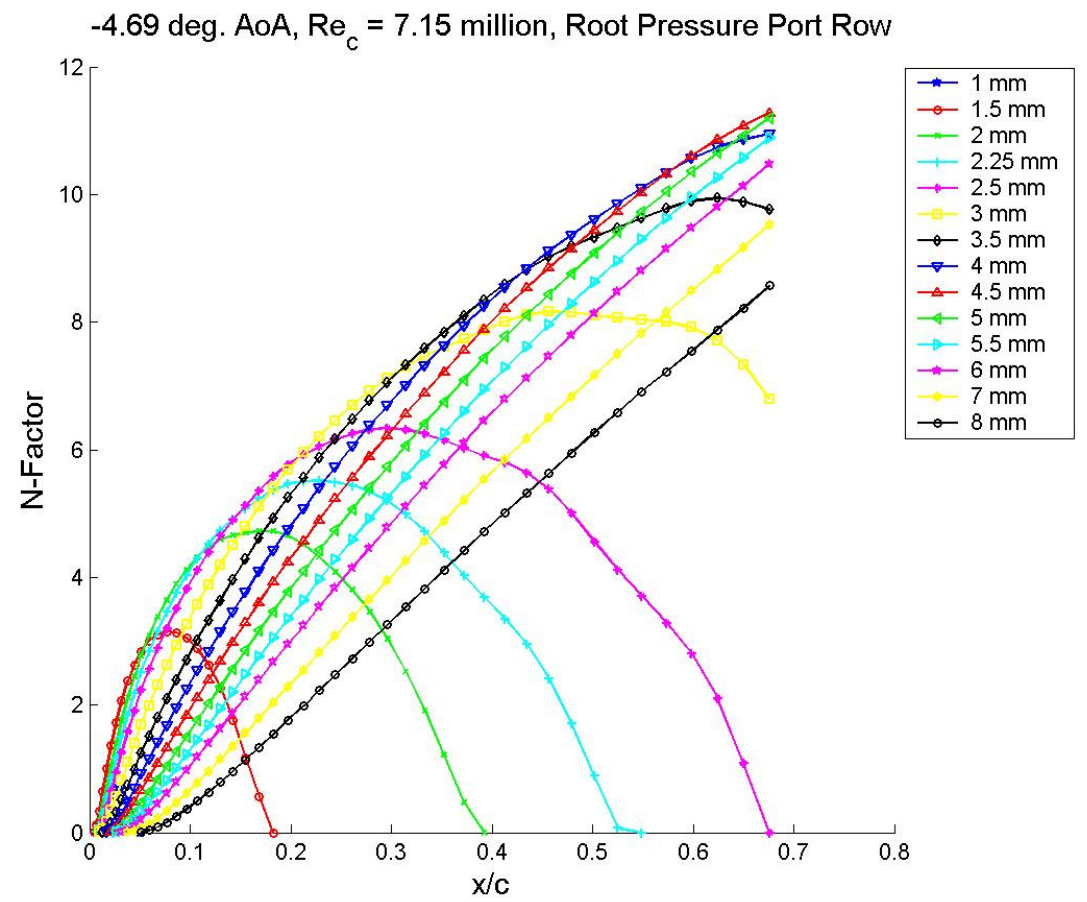

Figure 42: $N$-factors for Test Point 27 at -4.69 degrees $A o A$ along the root pressure port row. 


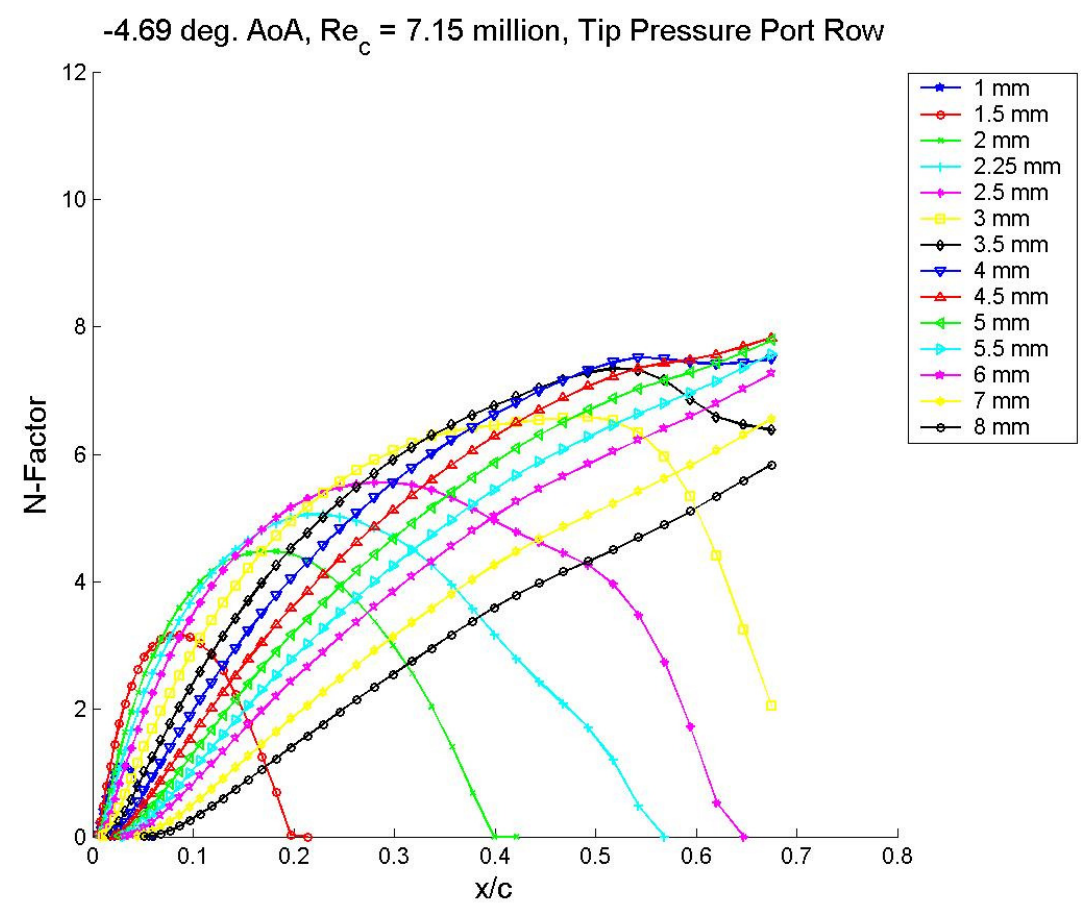

Figure 43: $N$-factors for Test Point 27 at -4.69 degrees $A o A$ along the tip pressure port row.

When examining the $N$-factor plots in Figures 40 through 43 , it is apparent that the $4.5 \mathrm{~mm}$ wavelength demonstrates the strongest growth over a wide range of test conditions. To test these calculations, DREs spaced $4.5 \mathrm{~mm}$ apart were placed at the calculated neutral point for the -2.61 degree $A o A$ test condition. This section focuses on this test condition because the baseline IR measurements demonstrated large amounts of NLF at this $A o A$. Thus the effectiveness of the critically-spaced DREs could be tested at this $A o A$ by promoting growth of the crossflow instability and observing any changes in the transition location with the IR camera. 


\subsubsection{Nonlinear Parabolized Stability Equations}

LST does a good job of determining the $\lambda_{\text {crit }}$, and neutral points, but fails to correctly determine the nonlinear shape of the disturbance growth. This effect was shown in the experiments of Reibert et al. (1996) and the calculations of Haynes \& Reed (2000). Nonlinear Parabolized Stability Equations (NPSE) can accurately match the nonlinear growth rate, but can also account for disturbances generated by roughness elements. This feature of the NPSE makes them ideally suited for demonstrating the effectiveness of DREs. A thorough review of NPSE calculations is given in Herbert (1997). In Figure 44, the stable test point at -2.61 degrees $A o A$ has been isolated for demonstrating the effect of adding roughness spaced $4.5 \mathrm{~mm}$ apart at different amplitudes. In the legend to the right there are different disturbance amplitude inputs, representative of roughness heights. However, the relationship between roughness heights and the disturbance amplitudes in Figure 44 is unknown. What is important in Figure 44, the disturbance amplitude $\mathrm{u}^{\prime}$ grows as the amplitude of the disturbance input is increased. If critically spaced DREs are placed at the neutral point and the roughness height is increased, then the disturbance amplitude also increases. If u' reaches a high enough amplitude, then early transition occurs due to leading edge roughness. 


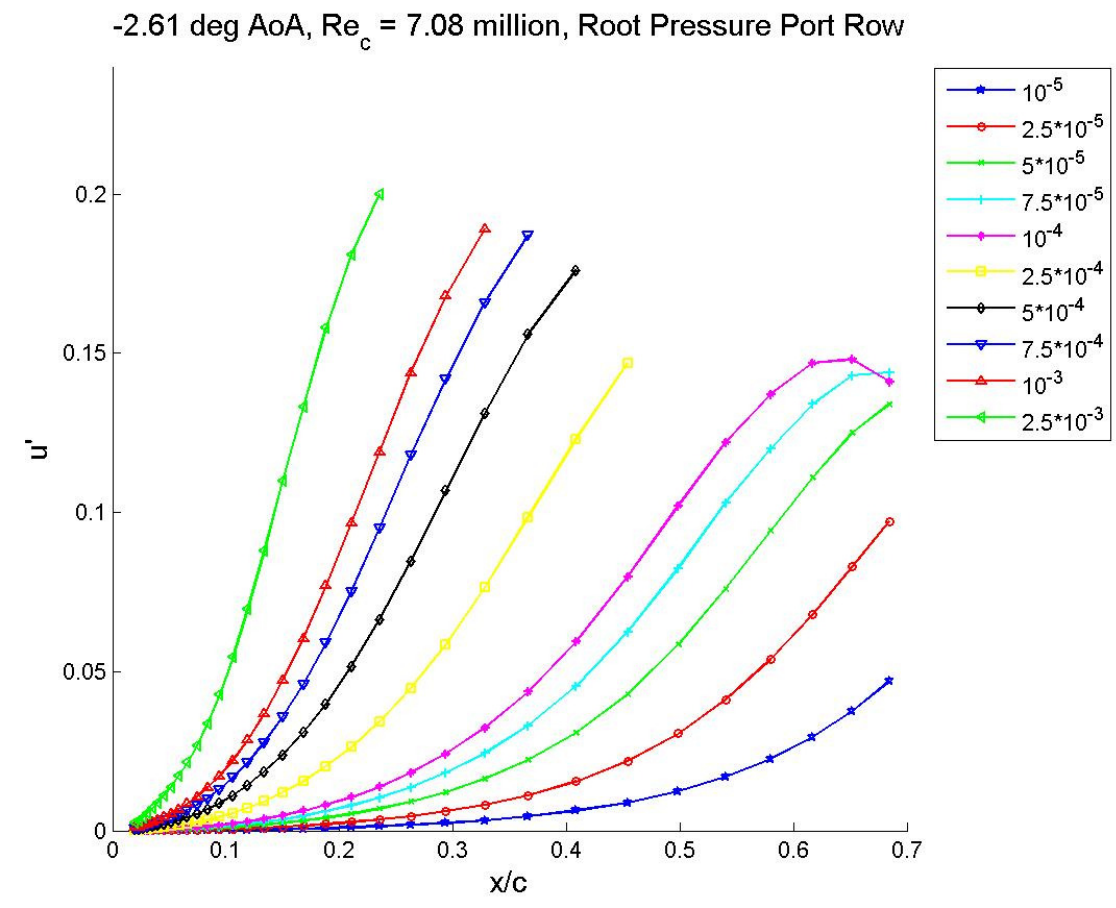

Figure 44: Disturbance amplitudes for Test Point 31 at -2.61 degrees AoA along the root pressure port row.

\subsection{Transition Results with Appliqué Critically Spaced DREs}

Test Point 31 at -2.61 degrees $A o A$ was chosen as a good test condition for demonstrating the effectiveness of the critically-spaced DREs. The goal was to excite the most unstable crossflow wavelength and move transition forward. At this moderately negative $A o A$, transition was observed at $80 \% x / c$ for both the painted and polished leading edge. For these experiments, the polished leading edge was used with the DRE insert and zero differential pressure applied. More information on the DRE insert will be provided in Section 6.5. Figure 45 shows the transition location for this condition. The flight number depicted in Figure 45 had DREs applied to the leading edge, but no effect 
on the transition location was observed. The baseline flight for this condition showed the same transition location, but the baseline flight produced poor IR images due to less than ideal atmospheric conditions. Thus, the IR image displayed in Figure 45 is used here as a representative baseline image, despite having DREs applied at the leading edge. Additionally, the $R e_{c}$ for this flight was lower than the targeted $R e_{c}$ of 7.5 million. This phase of the experiments was conducted during the summer when it was difficult to reach targeted $R e_{c}$ due to elevated temperatures. This limitation did not pose a problem because the success of the critically-spaced DREs would be more effective if transition moved forward under conditions of a lower $R e_{c}$. Figure 45 shows transition at $80 \% x / c$ for the area of interest in between the pressure port rows. The goal was then to take this condition and promote the growth of the crossflow instability by introducing periodic roughness at the most unstable vortex spacing. The IR camera was used to determine the change in transition location as a function of DRE height, $k$. 


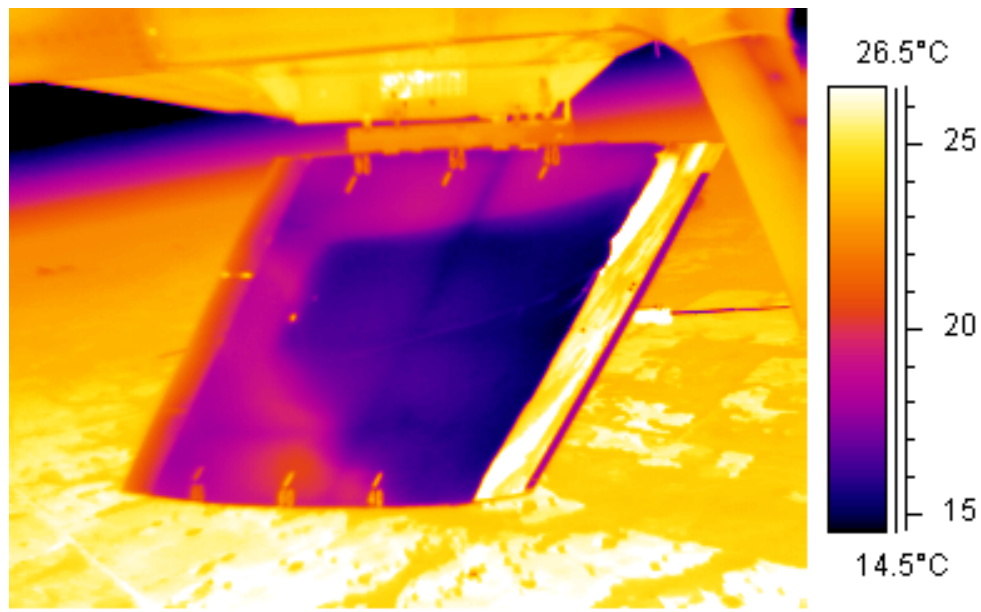

$$
\begin{array}{ll}
\text { AoA }=-2.42 \text { deg } & \text { Flight } N_{0}: 080208-1 \\
\operatorname{Re}_{c}=7.05 \text { million } & \text { Time: } 09: 40: 33
\end{array}
$$

Figure 45: Baseline IR image used to demonstrate critically-spaced DREs.

The surface roughness of the leading edge was measured as before, except in this case the roughness was measured over the DRE insert, where the DREs were applied, and over the polished aluminum downstream of the DRE insert. The surface roughness over the DRE insert was measured to be $1.24 \mu \mathrm{m}$ rms with average peak-to-peak values of $4.57 \mu \mathrm{m}$. The polished aluminum downstream of the DRE insert was measured to be $0.24 \mu \mathrm{m}$ rms with average peak-to-peak values of $1.00 \mu \mathrm{m}$.

The LST calculations showed that one of the most unstable crossflow vortex spacing was $4.5 \mathrm{~mm}$. Roughness elements were placed $4.5 \mathrm{~mm}$ apart at the calculated neutral point of $2.21 \% x / c$ at the root pressure port row and $2.41 \% x / c$ at the tip pressure port row. The calculations showed that the spanwise variation in the $C p$ distribution resulted in a spanwise variation in the neutral point. Under these flight conditions, the 
root pressure port row always had a neutral point closer to the attachment line. As a result, the DREs were placed along a line that was not at a constant $x / c$, but rather shifted downstream at the tip to account for the spanwise variation. Figure 46 shows the configuration used for all flights incorporating DREs at the leading edge. In Figure 46 the DREs are shown along the entire span of the model. The first and last DRE behave as an isolated $3-\mathrm{D}$ roughness which introduces a wave packet containing $\lambda_{\text {crit }}$ (Radeztsky et al. 1993a). As a result, the endpoints may produce turbulent wedges contaminating the area of interest. To avoid such turbulent wedges, the DREs are applied along the entire span of the leading edge to reduce end effects.

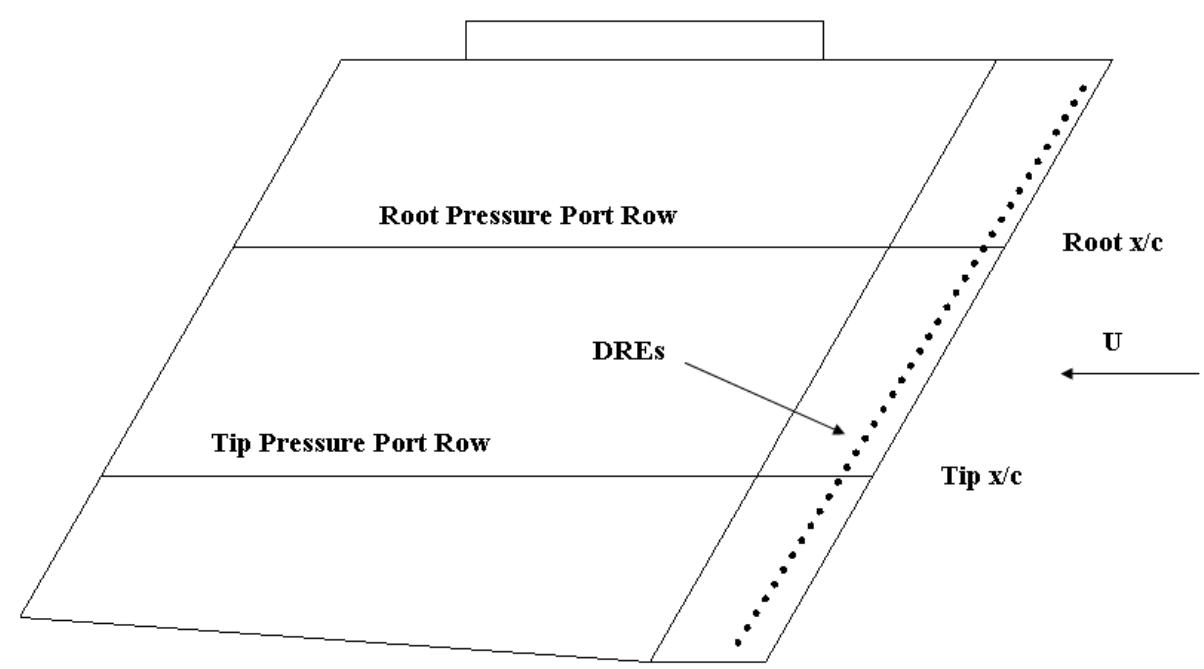

Note: DREs not drawn to scale

Figure 46: Depiction of DREs applied to the leading edge with a different $x / c$ location at the root and tip pressure port rows. 
The roughness elements used during this part of the experiment will be referred as appliqué DREs. They are transfer elements commonly used in the graphic arts industry. Each element is idealized as right-circular cylinders with minimum heights of 6 $\mu \mathrm{m}$. Increased roughness heights are created by stacking individual elements.

In addition to specifying the roughness height, $k$, it is instructive to present roughness data with a Reynolds number based on roughness height, $R e_{k}$. which is calculated using the velocity, $u_{k}$, and the kinematic viscosity, $v_{k}$, at the roughness height. Equation 7 shows the relationship for $R e_{k}$.

$$
R e_{k}=\frac{u_{k} k}{v_{k}}
$$

Furthermore, the roughness height can be represented as a fraction of the boundary-layer height, $k / \delta$. Figure 47 shows a plot of calculated boundary layer heights for both the root and tip pressure port rows for Test Point 27 at -4.69 degrees AoA. The boundary-layer heights for Test Point 31 are close to those for Test Point 27 tested here. Therefore, Figure 47 is used here to represent the magnitude of the boundary-layer height under these $R e_{c}$ conditions. $k, R e_{k}$, and $k / \delta$ are supplied for all figures incorporating DREs as a reference for the heights of the DREs. 


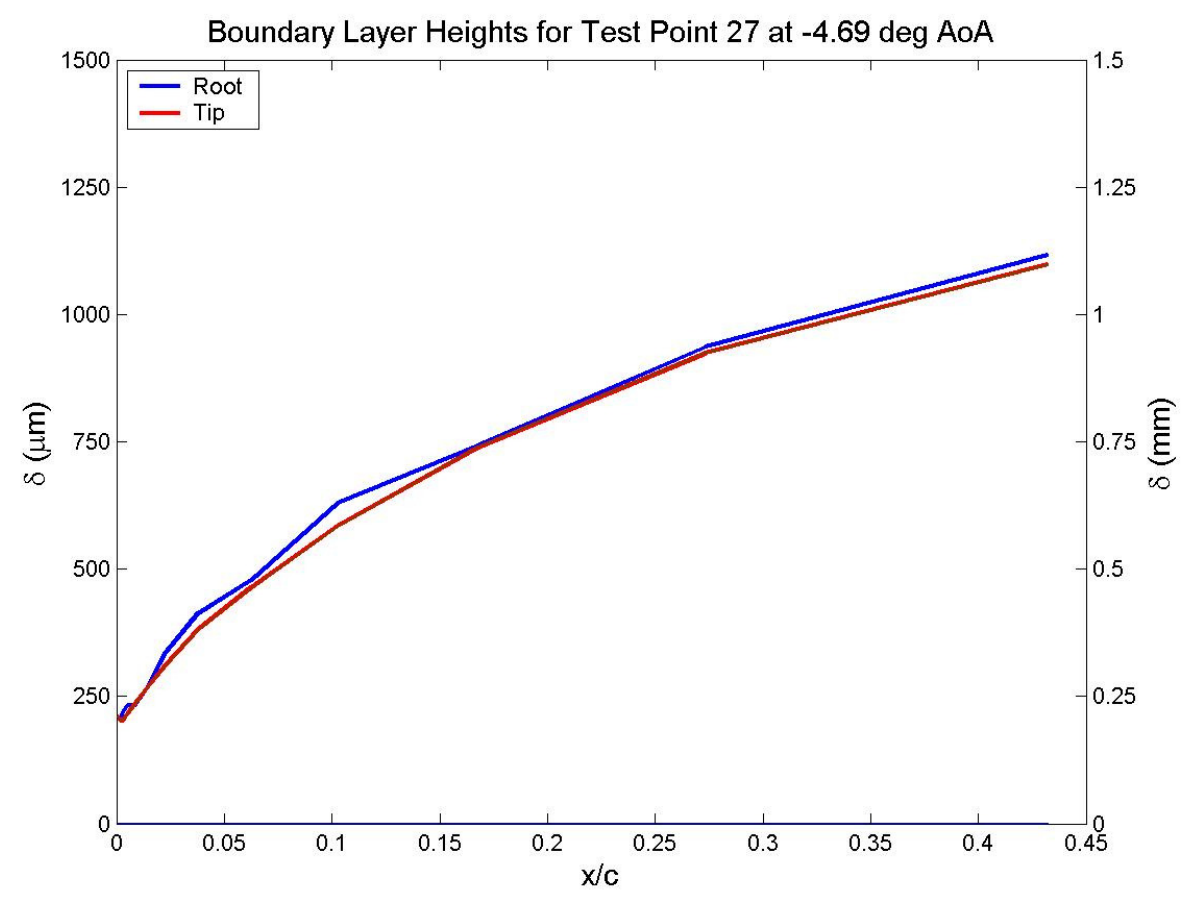

Figure 47: Plot of root and tip boundary-layer heights for Test Point 27.

Flights were conducted at these $x / c$ locations until the DREs reached heights of $30 \mu \mathrm{m}$. At roughness heights of $30 \mu \mathrm{m}$, only the bottom half of the model showed signs of early transition. This was indicative that the neutral point calculation for the tip was correct, but the root DRE location required some adjustment. As a result, the DREs at the root pressure port row were shifted forward to $2.07 \% x / c$. The procedure was then repeated and explained in more detail here.

A full-span array of critically-spaced DREs was applied to the leading edge at the $2.07 \% x / c$ root location and the $2.41 \% x / c$ tip location. The roughness elements used for these experiments had a height of $k=6 \mu \mathrm{m}$ and a diameter of $d=1.1 \mathrm{~mm}$. The notation 
for representing roughness statistics established in Reibert et al. (1996) is also employed here, with the exception that the roughness diameter is also added for greater clarity. Roughness data are presented as $[k|\lambda| d]$ in all figures, where $k$ is in $\mu \mathrm{m}, \lambda$ is in $\mathrm{mm}$, and $d$ is in $\mathrm{mm}$. The spacing between each roughness element was $\lambda=4.5 \mathrm{~mm}$ during this part of the experiments. For the $6 \mu \mathrm{m}$ case there was no change and transition was again observed at $80 \% x / c$, as in Figure 45. An additional layer of roughness elements was applied up to $12 \mu \mathrm{m}$, again with the result that no change in transition was observed. This procedure was repeated until $k$ equaled $24 \mu \mathrm{m}$ and the section of the model below the tip pressure port row became fully turbulent at all $A o A s$ tested and all $\operatorname{Re}_{c}$ observable with the IR camera. The top region eventually became turbulent, but only at a more unstable negative AoA. Furthermore, the turbulent region on the upper half of the model never moved forward of the $15 \% x / c$ location, as the bottom half had done with the DREs. The turbulent region in Figure 48 appears to be very close to the attachment line. However, the leading edge was polished aluminum and very little detail can be recovered forward of $15 \% x / c$ with the IR camera. Therefore, it is unknown where the actual transition location was and only an estimate of less than $15 \% x / c$ can be made. 


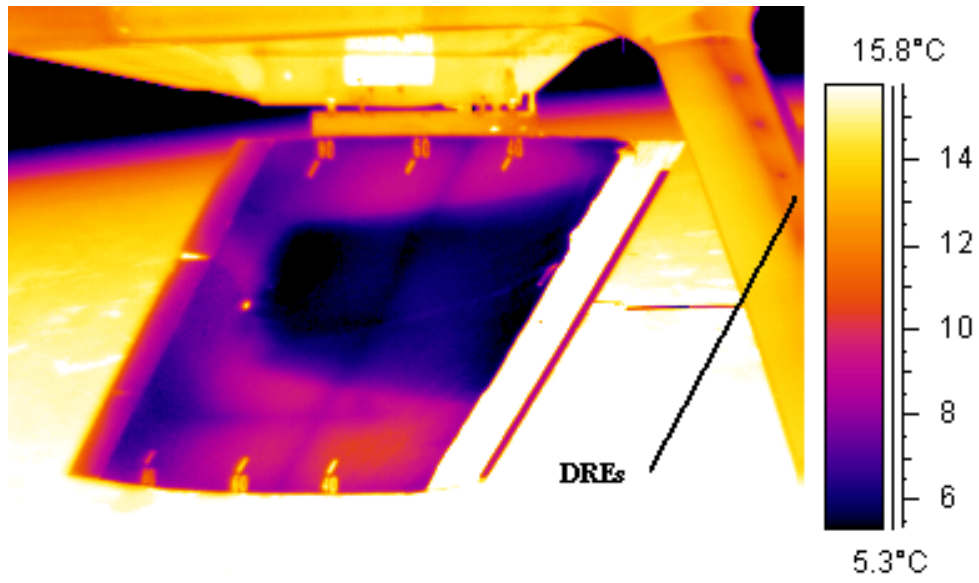

$\mathrm{AoA}=-3.28 \mathrm{deg}$

Flight No: 051808-1 $\operatorname{Re}_{\mathbf{c}}=7.26$ million

Time: 10:20:55

Figure 48: IR image with [24|4.5|1.1] $\left(\operatorname{Root} R e_{k}=8.8\right.$, Tip $R e_{k}=10.1, k / \delta_{\text {root }}=0.08$, $\left.k / \delta_{\text {tip }}=0.08\right)$ critically-spaced DREs.

The DRE heights were then increased to $30 \mu \mathrm{m}$, but not along the entire span. The critical DREs were placed at the locations indicated in Figure 49. In these two regions, transition occurred very close to the DREs, i.e. less than $15 \% x / c$. In between the two sections of DREs were isolated roughness elements with heights of $42 \mu \mathrm{m}$. These isolated roughness elements were placed in the two laminar regions depicted in Figure 49. In light of Figure 49, it appears as though the isolated roughness elements are less effective than the periodic array of roughness at exciting the crossflow instability. The areas with isolated roughness elements showed no sign of early transition, despite having higher roughness heights at $42 \mu \mathrm{m}$. On the other hand, the region downstream of the $30 \mu \mathrm{m}$ DREs was turbulent. Based on the success of the $30 \mu \mathrm{m}$ DREs, the entire span was filled in at the $2.07 \%$ root $x / c$ location and $2.41 \%$ tip $x / c$ location. Under these 
conditions the model was turbulent for all $R e_{c}$ values recorded with the IR camera. This condition is shown in Figure 50.

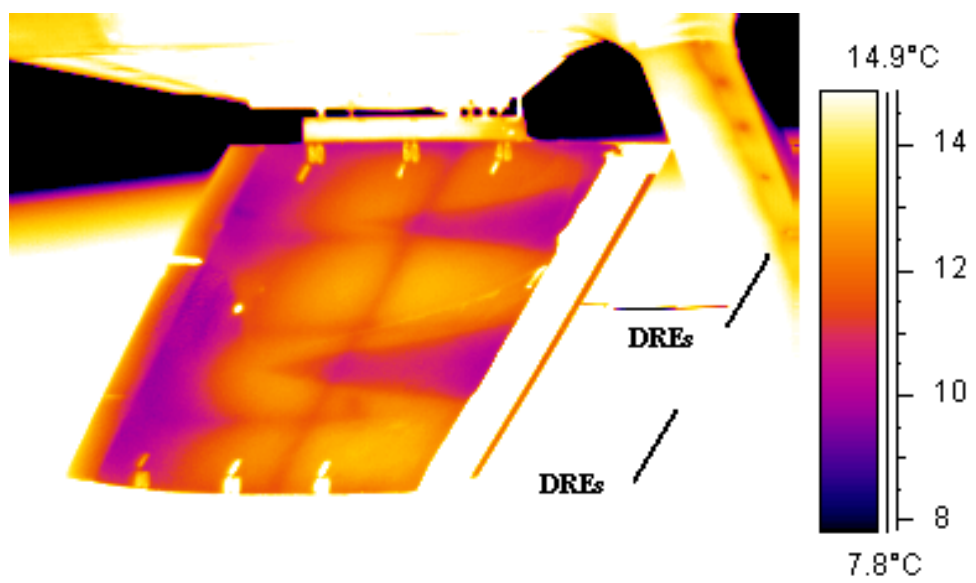

$$
\begin{array}{ll}
\mathrm{AoA}=-2.65 \mathrm{deg} & \text { Flight } \mathrm{No}_{0} 051908-2 \\
\operatorname{Re}_{\mathbf{c}}=7.15 \text { million } & \text { Time: 10:01:08 }
\end{array}
$$

Figure 49: IR image with [30|4.5|1.1] (Root $R e_{k}=14.1$, Tip $R e_{k}=16.3, k / \delta_{\text {root }}=0.10$, $\left.k / \delta_{\text {tip }}=0.10\right)$ critical DREs and $42 \mu \mathrm{m}\left(\operatorname{Root} R e_{k}=28.4\right.$, Tip $R e_{k}=33.1, k / \delta_{\text {root }}=0.13$, $k / \delta_{\text {tip }}=0.14$ ) isolated roughness elements in between the critically-spaced DREs. 


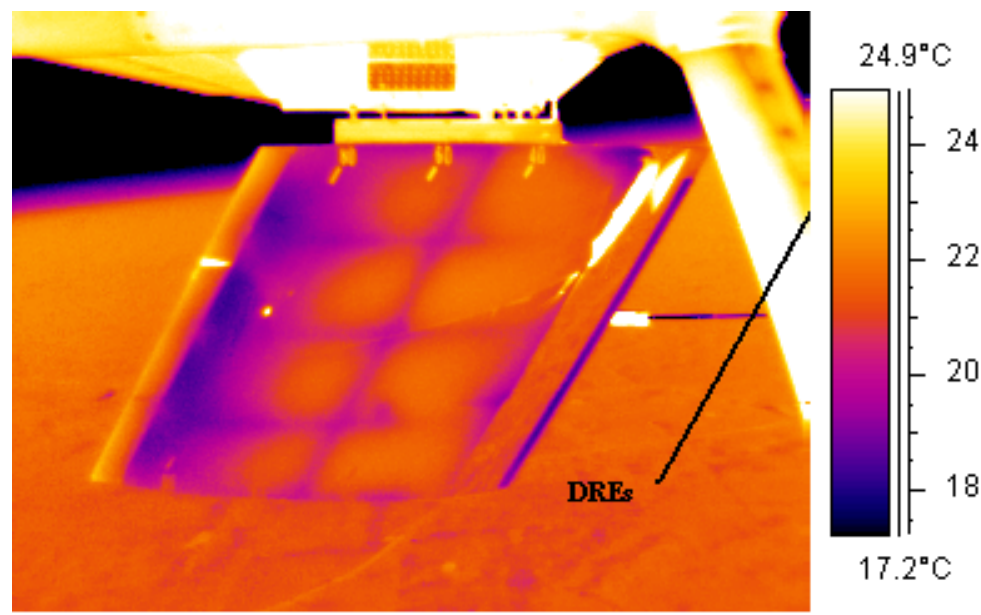

$$
\begin{array}{ll}
\mathrm{AoA}=-2.55 \mathrm{deg} & \text { Flight } \mathrm{No:} 052008-1 \\
\operatorname{Re}_{c}=7.37 \text { million } & \text { Time: } 07: 30: 53
\end{array}
$$

Figure 50: IR image with [30|4.5|1.1] (Root $R e_{k}=14.1$, Tip $R e_{k}=16.3, k / \delta_{\text {root }}=0.10$, $\left.k / \delta_{\text {tip }}=0.10\right)$ critically-spaced DREs.

For Figures 48 through 50 it is worth mentioning that each of these IR images were unchanged throughout the dive. In each case, transition was observable at approximately 7 million $R e_{c}$ and the image looked identical to the ones displayed here. Therefore, the DREs spaced at $4.5 \mathrm{~mm}$ appear to be effective at destabilizing the crossflow instability over a wide range of $R e_{c}$ values. Additionally, during these three flights the pilot was instructed to change $A o A$ on the model. During these $A o A$ excursions the transition pattern did not change, also indicating that the $4.5 \mathrm{~mm}$ stationary crossflow vortices were destabilizing over a large range of test conditions. Figure 51 shows an IR image of Flight No: 052008-1 during the climb. This flight corresponds to Figure 50 which had DREs at $k=30 \mu \mathrm{m}$. Figure 51 shows that the model was laminar at the lower $R e_{c}$ values, indicating that the critical DREs were only effective 
above a minimum $R e_{c}$ value. Note that in Figure 51 the IR image is reversed in colors. The colder region is turbulent, whereas the warmer region is laminar. This reversal is because the model is being cooled during the climb, so the turbulent boundary layer is cooling the model at a faster rate. It was routine for the FTE to record the IR video during the climb in order to identify turbulent wedges from bug strikes or bad DREs. In this case, the mission would be aborted so the leading edge could be wiped clean or the DREs could be repaired.
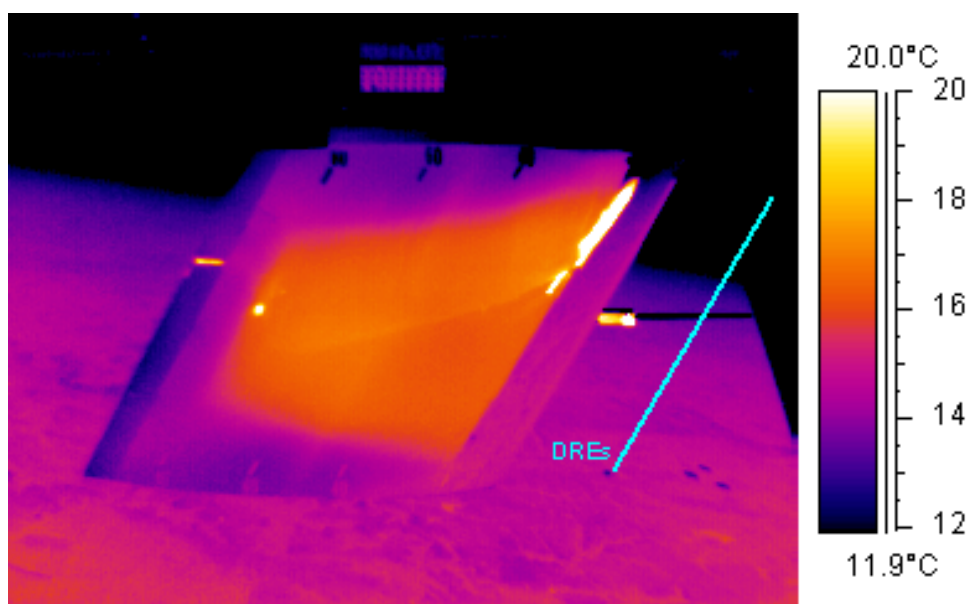

$$
\begin{array}{ll}
\mathrm{AoA}=-1.79 \mathrm{deg} & \text { Flight } \mathrm{No}_{0}: 052008-1 \\
\operatorname{Re}_{c}=3.18 \text { million } & \text { Time: 06:46:13 }
\end{array}
$$

Figure 51: IR image of the climb with [30|4.5|1.1] (Root $\operatorname{Re}_{k}=14.1, \operatorname{Tip} R e_{k}=16.3$,

$$
\left.k / \delta_{\text {root }}=0.10, k / \delta_{\text {tip }}=0.10\right) \text { critically-spaced DREs. }
$$

\subsection{Effect of Roughness Diameter}

All of the flights depicted in Figures 48 through 51 had DREs with a diameter of $d=1.1 \mathrm{~mm}$ and a spacing of $\lambda=4.5 \mathrm{~mm}$. This resulted in a ratio of $d / \lambda=0.24$. Using 
this configuration, the critical DREs appeared to be effective once the height reached somewhere between $24 \mu \mathrm{m}$ to $30 \mu \mathrm{m}$. At lower heights, no change in transition was observed. However, Radeztsky et al. (1993a) showed that the most effective $d / \lambda$ ratios were on the order of 0.40 and 0.50 . As a result, larger $d / \lambda$ ratios were tested here to compare with these earlier results. A small segment of the model was tested with 4.5 mm-spaced DREs with diameters of $2.3 \mathrm{~mm}$. With a $d / \lambda$ ratio of 0.51 , these DREs were expected to be more effective than the $1.1 \mathrm{~mm}$ diameter DREs. The first height tested was $6 \mu \mathrm{m}$, but no sign of early transition was observed. At $12 \mu \mathrm{m}$, the region downstream of these larger diameter DREs was turbulent forward of $15 \% \mathrm{x} / \mathrm{c}$. These larger DREs were also placed along the same $x / c$ line of $2.07 \%$ at the root, and $2.41 \%$ at the tip. The early transition observed with the $2.3 \mathrm{~mm}$ DREs agrees with the findings of Radeztsky et al. (1993a) that a $d / \lambda$ ratio of 0.4 to 0.5 is most effective at introducing streamwise vorticity to excite the crossflow instability.

\subsection{Design of Variable Height Roughness Elements}

The behavior exhibited with the appliqué roughness elements indicated that there was some critical height leading to the magnification of $\lambda_{\text {crit }}$. A gradual change in transition was never observed here. Transition was either observed at $80 \% x / c$ or forward of $15 \% x / c$. As a result, a design was initiated for changing the roughness heights in a continuous manner and not only at discrete 6 - $\mu \mathrm{m}$ increments. Furthermore, it was desired to have a control system that could change the heights of the DREs while in 
flight. This required a design change of the leading edge. The design used here was first demonstrated with White \& Saric (2000) and proved to be effective at modifying transition. The idea was to create a pressurized chamber where a flexible membrane would seal off holes at the top of the surface. As pressure is applied to the chamber, the flexible membrane would stretch and generate a roughness element. The height of the element was proportional to the applied pressure and could be controlled in flight. Additionally, vacuum could be applied for generating variable-height dimples. This type of roughness element is referred to as variable-height DREs.

Leading edge \#2 was modified to accept an insert that nearly covered the entire span of the leading edge. A pocket was milled out of the leading edge to hold various DRE inserts. The pocket was $2.7 \% x / c$ thick with the leading edge of the insert at $0.6 \%$ $x / c$ and the trailing edge at $3.3 \% x / c$. The insert was composed of two parts. The top surface matched the shape of the leading-edge curvature. The underside of the top surface incorporated a pressure chamber that was filled with positive or negative gauge pressure. The outer surface of the top piece was drilled with small-diameter holes to create the circular cross-sectional shape of the roughness element. A layer of $48 \mu \mathrm{m}$ thick Polyester-film tape was applied over the surface to seal off the top surface and act as the flexible membrane for the variable-height DREs. The second part was a flat plate with an O-ring to seal off the bottom of the pressurized chamber. The insert assembly was intentionally designed to sit below the surface of the airfoil in order to account for the tape thickness. A pipe fitting at the trailing edge of the insert provided supply 
pressure for positive-height DREs, or vacuum for creating dimples. A pressure port was added to the leading edge for a static pressure reference. Figure 52 is a schematic of the modified leading edge with the DRE insert.

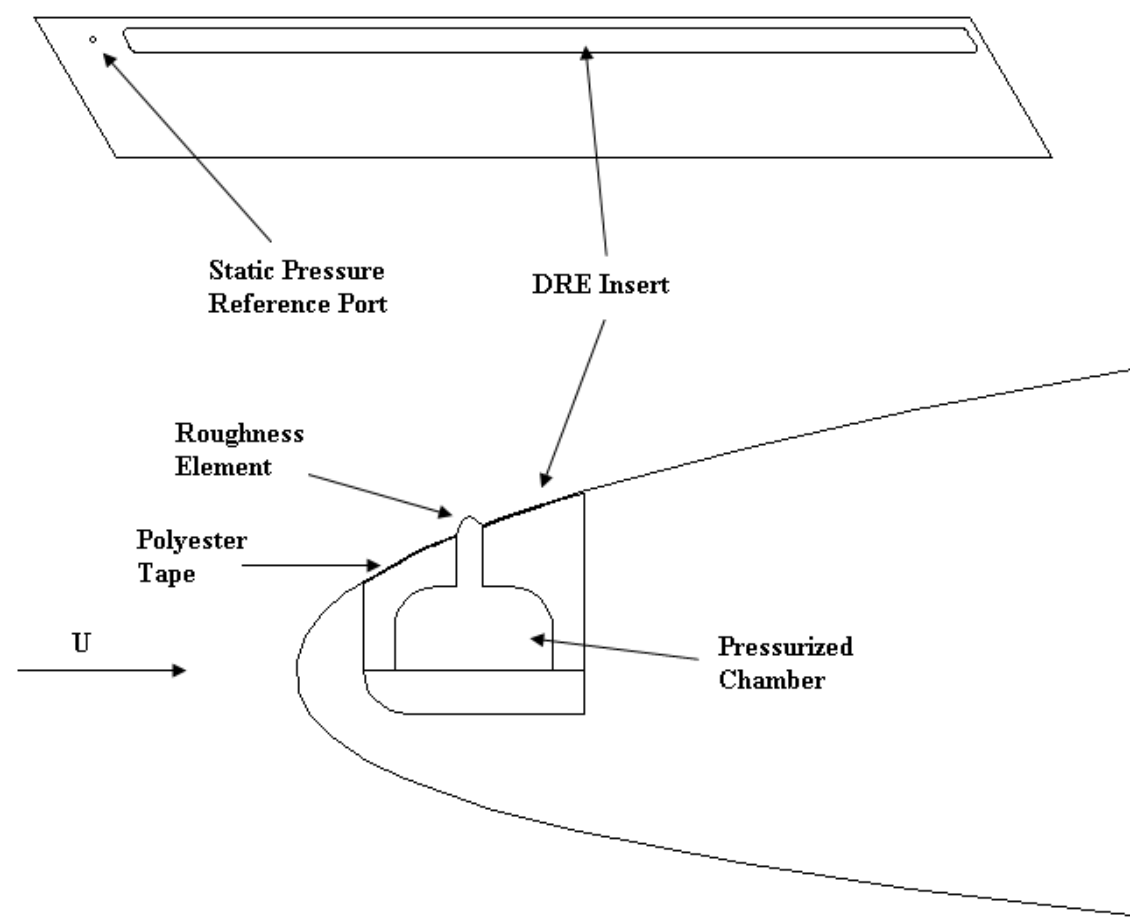

Figure 52: Schematic of DRE insert assembly.

An electronic pressure regulator, capable of both pressure and vacuum control, was purchased to drive this pneumatic system. The LJ Engineering 487-002 selfrelieving electronic regulator met these specifications. This regulator can supply either pressure or vacuum up to $15 \mathrm{psig}$. The electronic regulator was controlled by a 0 to 5 VDC signal from the flight computer. In order to switch from vacuum mode to pressure mode, the supply and reference lines needed to be switched on the regulator. To 
accomplish this in flight, a four-way valve, accessible to the FTE, was installed to switch the two pressure lines. To supply pressure to the regulator, a one-gallon air tank was assembled next to the regulator. Before each flight, the air tank was filled to 90 psig. Because no significant mass flow was used in the system, the one-gallon tank could supply pressure to the DRE insert for several hours before requiring a refill. On the other hand, when applying vacuum, the tank could only supply enough vacuum for a few minutes of operation. As a result, the control system was modified to incorporate a vacuum pump with an automatic vacuum switch and check valve for maintaining the required vacuum in the supply tank. Typically a flight was either dedicated as a pressure flight or a vacuum flight. However, the entire assembly could be switched in flight from pressure to vacuum in less than five minutes. The first dive was dedicated as a pressure run. During the climb back to altitude, the FTE would deplete the pressure in the tank, turn on the vacuum pump, and then switch the four-way valve going to the regulator. The reverse operation was not an option because the compressed air side of the pump was never connected to the tank in order to simplify the design. The entire assembly weighed $32 \mathrm{lbs}$ and was mounted on the instrumentation rack behind the FTE.

The supply lines going to the leading edge were all $1 / 8$ " inner diameter, vacuumrated Tygon tubing. The supply pressure going to the insert was referenced to the static pressure port on the leading edge, as depicted in Figure 52. This pressure port was above the DRE insert, and at the same $x / c$ location as the DREs. The static pressure port allowed calibration of the DRE heights as a function of supply pressure on the ground, 
yet the calibration was still valid at altitude. Provided all calibrations were performed with respect to the pressure reference, the calibrations would be valid for any ambient pressure. The procedure for calibrating the pneumatic DREs with the confocal laser was described in Section 2.11.2. Figure 53 shows three example calibration curves for three different hole diameters. Both the $1.1 \mathrm{~mm}$ and $2 \mathrm{~mm}$ DREs were tested with the critical spacing of $\lambda_{\text {crit }}=4.5 \mathrm{~mm}$. The $1-\mathrm{mm}$ diameter DREs were used for the control case, which is described in Section 8. A third-order polynomial curve fit, with the intercept set to zero, was used for all calibration curves. Typically three random DREs were chosen to perform a calibration to test for repeatability. There was usually some variation between the three calibration curves. The variation was due to difficulty in locating the center of the variable-height roughness element rather than strength variations in the tape. This is assumed because the variation in calibration curves got smaller with the larger $2 \mathrm{~mm}$ holes, where the roughness center was easier to locate. Note that there is a discontinuity in Figure 53 at zero pressure. This discontinuity occurs because the pneumatic control system cannot continuously switch between vacuum and pressure. The acquisition must be stopped for the system to be switched between vacuum and pressure, resulting in a discontinuity at zero differential pressure. 


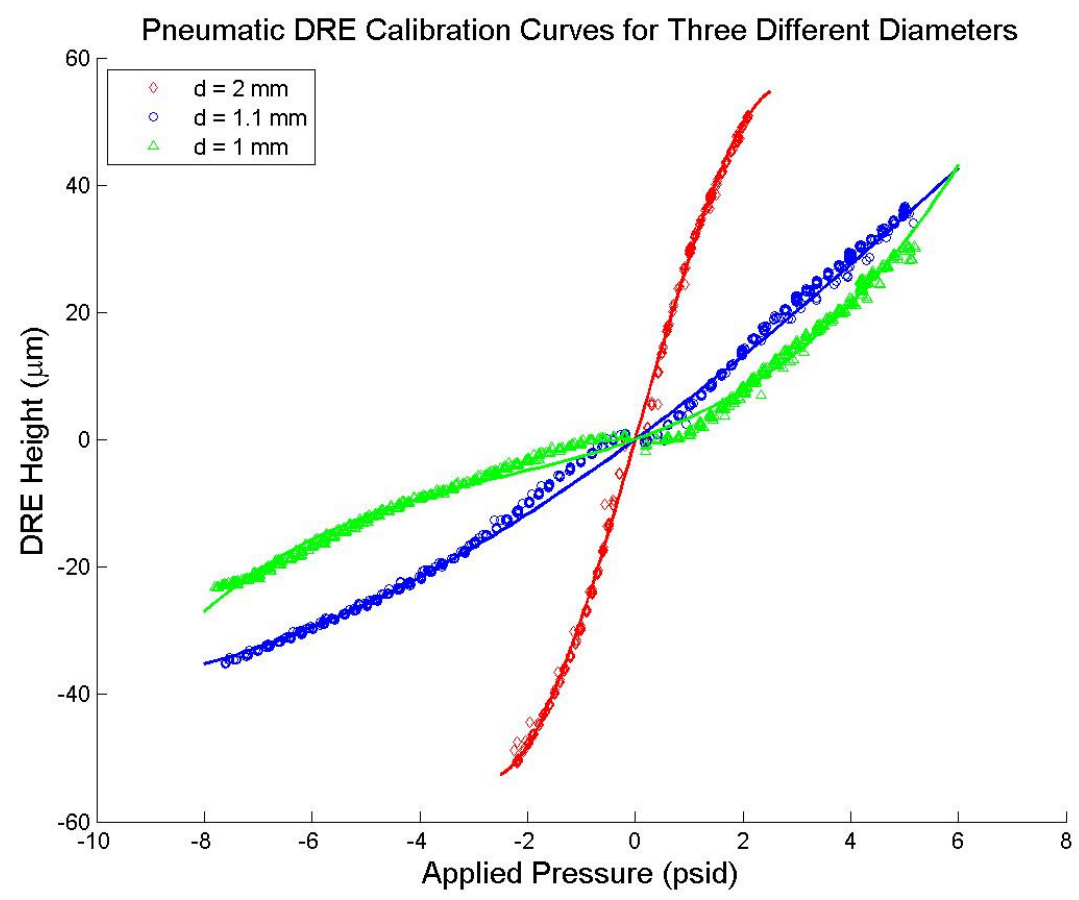

Figure 53: Example of variable-height DRE calibration curves measured at the center of each roughness element.

\subsection{Transition Results with Variable Height Critical DREs}

Based on the results obtained with the appliqué DREs, one of the DRE inserts was drilled to match the conditions already tested with the appliqué DREs. The insert was drilled with $1.1 \mathrm{~mm}$ holes, spaced $4.5 \mathrm{~mm}$ apart, and at the $2.07 \% x / \mathrm{c}$ root and $2.41 \% x / c$ tip location. It was expected that early transition would be observed somewhere between 24 and $30 \mu \mathrm{m}$, as was observed with the appliqué DREs. With the variable-height DREs, the height could be held in between this range to see if transition could be manipulated in a more controlled manner. Figure 54 shows an IR image with the DREs turned off and Figure 55 shows an IR image with the DREs at $k=31 \mu \mathrm{m}$. No 
change in transition was observed after pressurizing the DREs. The heights were then pushed beyond what was calibrated to an extrapolated DRE height of $75 \mu \mathrm{m}$. This condition is shown in Figure 56. Even under these extreme roughness heights, no change in transition was observed. While the DREs remained pressurized, the pilot was instructed to decrease the $A o A$. Transition moved forward, but only due to the destabilizing nature of the natural crossflow at decreased $A o A$ values. The transition front never moved forward of the $15 \% x / c$ location, which it had before with the appliqué DREs. The same was true after trying more stable conditions at increased AoAs where transition remained at $80 \% x / c$ for this case. These results were contrary to the results obtained earlier with the appliqué DREs. The holes were the same diameter, in the same location, and heights were pushed beyond what was tested with the appliqué DREs; yet, no change in transition was observed. Vacuum was also applied down to -60 $\mu \mathrm{m}$, but still no change in transition was recorded. 


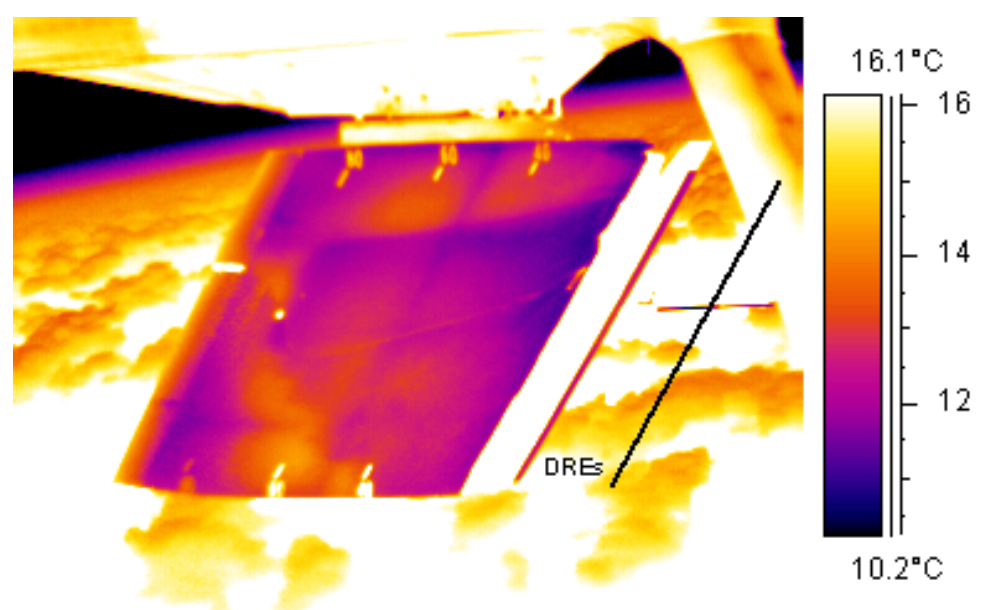

$$
\begin{array}{ll}
\mathrm{AoA}=-2.60 \mathrm{deg} & \text { Flight } \mathrm{No:}: 061408-1 \\
\operatorname{Re}_{c}=7.05 \text { million } & \text { Time: } 11: 42: 14
\end{array}
$$

Figure 54: Baseline IR image with polished leading edge and DRE insert with no pressure applied.

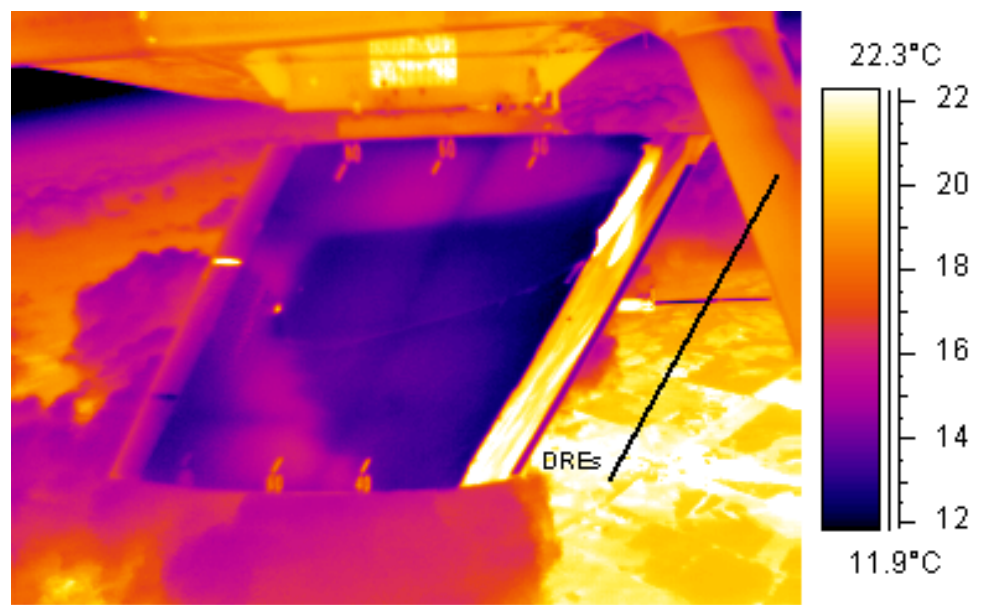

$$
\begin{array}{ll}
\text { AoA }=-2.54 \text { deg } & \text { Flight } N_{0}: 061408-1 \\
\operatorname{Re}_{c}=7.32 \text { million } & \text { Time: } 11: 42: 42
\end{array}
$$

Figure 55: IR image with [31|4.5|1.1] (Root $R e_{k}=15.1$, Tip $R e_{k}=17.4, k / \delta_{\text {root }}=0.10$, $\left.k / \delta_{\text {tip }}=0.10\right)$ critically-spaced, variable-height DREs. 


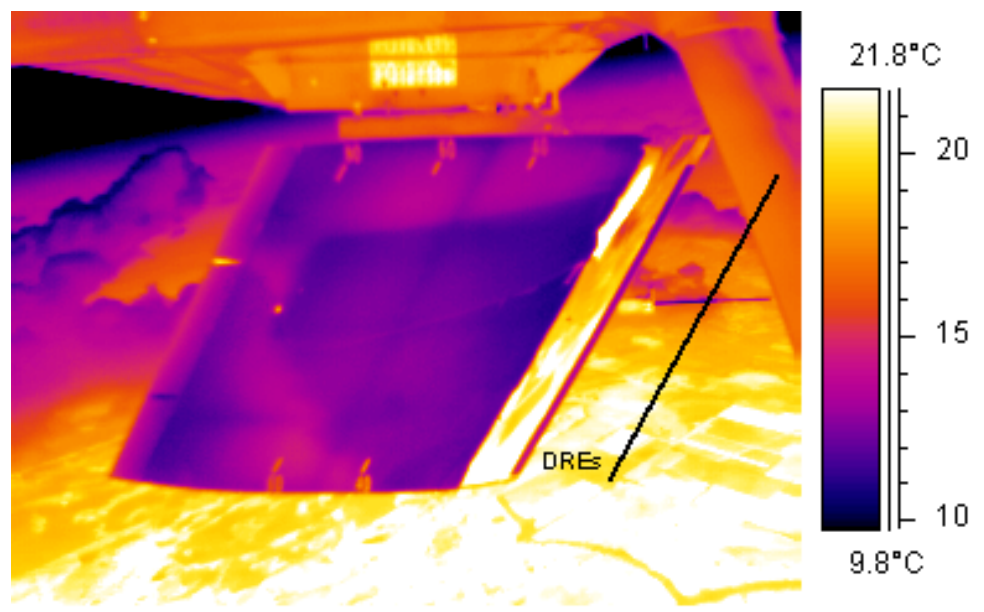

$$
\begin{array}{ll}
\mathrm{AoA}=-2.54 \text { deg } & \text { Flight } \mathrm{No}_{0} \text { 061408-1 } \\
\operatorname{Re}_{\mathrm{c}}=7.21 \text { million } & \text { Time: } 12: 35: 09
\end{array}
$$

Figure 56: IR image with [75|4.5|1.1] $\left(\operatorname{Root} R e_{k}=93.4\right.$, Tip $R e_{k}=109.8, k / \delta_{\text {root }}=0.24$, $k / \delta_{\text {tip }}=0.24$ ) critically-spaced, variable-height DREs.

A suggestion was made to paint the leading edge in an attempt to introduce additional roughness and vorticity that might interact with the variable-height DREs. First, the leading edge and DRE insert were painted separately and then assembled. The same procedure of omitting the thinning agent in the paint, as described in Section 5, was also used here. However, no change in transition was observed. The paint introduced non-uniformities in the DRE heights in addition to inhibiting the growth of $k$ from the added material thickness of the paint. Furthermore, it was difficult to visually see the roughness elements when white paint was applied over the normally reflective Polyester-film tape. This paint made it difficult to locate the DRE center with the confocal laser for height calibration. In light of these difficulties, the paint was removed 
from the DRE insert, but the paint remained on the rest of the leading edge. This setup also failed and transition did not move forward under similar flight conditions.

Another suggestion was made to increase the hole diameter. When one looks at the cross-section of the variable-height DREs, there is a difference between the variableheight DREs and the appliqué DREs. Figure 57 shows DRE scans with the Mitutoyo surface-roughness tester of the two different DRE types tested. The appliqué DREs are idealized as a right-circular cylinder whereas the variable-height DREs are shaped more like a Gaussian curve. Figure 57 also shows the vacuum applied dimple has a larger $k$ than the pressure applied bump. This is because the surface roughness tester scans the surface with a stylus. The stylus pushes down on the surface with a small force of 0.75 $\mathrm{mN}$. This force is not large enough to scratch a polished surface, but is large enough to deflect the tape under all pressure settings. Furthermore, the stylus drags through the surface of the appliqué DREs which removes some of the material, as seen after viewing the appliqué DREs under a microscope. Despite these difficulties, the intent of using the Mitutoyo surface roughness tester was to get an idea of the cross-sectional shape and not the absolute height, $k$. The confocal laser was used for measuring $k$, but the surface roughness tester did a good job of scanning the cross-sectional shape.

Note that in Figure 57 the appliqué roughness element is $1.1 \mathrm{~mm}$ in diameter, however the scans show a diameter closer to $1.5 \mathrm{~mm}$. This is due to inconsistencies in stacking the appliqué DREs on top of one another. To reach a height of $30 \mu \mathrm{m}$ with the appliqué DREs requires five layers of DREs. As the number of layers increases, the 
diameter of the overall roughness element increases proportionally. Nevertheless, the cross-sectional scans still show a nearly vertical edge, which is not seen with the variable-height DREs.

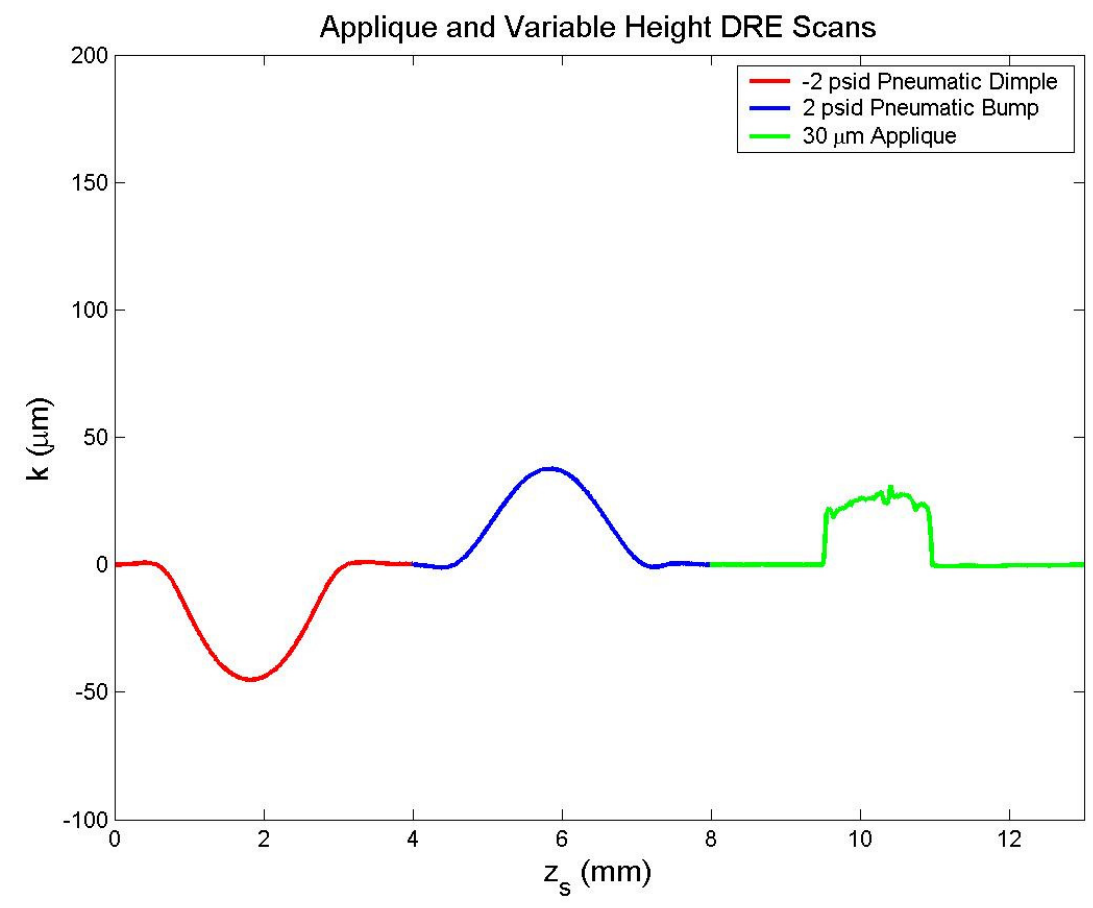

Figure 57: Schematic of $2 \mathrm{~mm}$ diameter, variable-height DREs and an appliqué DRE with a diameter of $1.1 \mathrm{~mm}$.

It was believed that there might be an effective roughness diameter that is less than the hole diameter drilled into the surface. Therefore, the $1.1 \mathrm{~mm}$ appliqué DREs were effective at moving transition, but the variable-height DREs had a $d / \lambda$ ratio too small to be effective. Figure 58 shows a depiction of the possible effective diameter of a $2 \mathrm{~mm}$ variable-height DRE. For this case the effective diameter is smaller than the $2 \mathrm{~mm}$ 
hole drilled into the surface, possibly making it less effective. As a result, a twelve inch section of the holes centered on the root pressure port row were drilled from $1.1 \mathrm{~mm}$ to 2 $\mathrm{mm}$ diameter. This resulted in a $d / \lambda$ ratio of 0.44 , which should have been large enough to be effective. Unfortunately, no change in transition was observed with the $2 \mathrm{~mm}$ holes and $k$ values ranging from $75 \mu \mathrm{m}$ with pressure applied and $-60 \mu \mathrm{m}$ with vacuum.

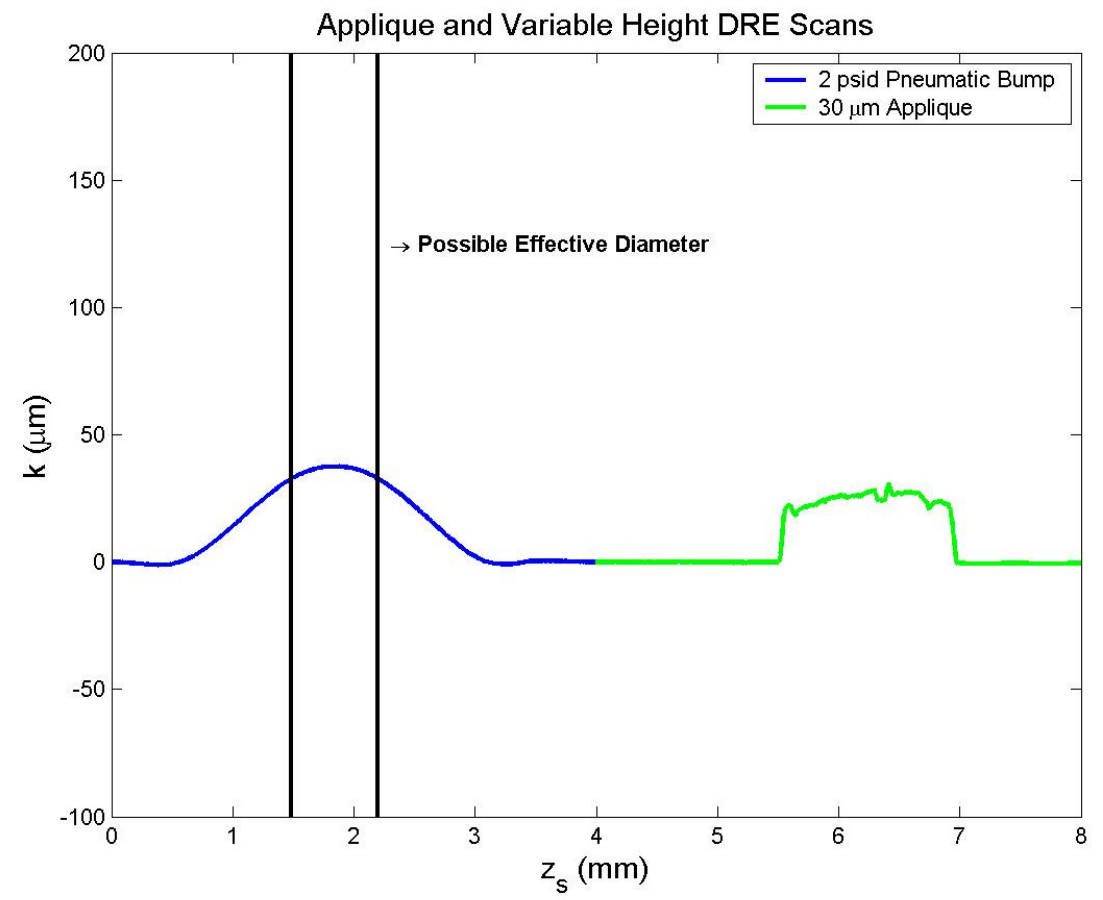

Figure 58: Schematic of a variable-height DRE and an appliqué DRE indicating the effective diameter theory.

After receiving the same lack of results with the $2 \mathrm{~mm}$ holes, it became apparent that the shape of the roughness element plays a very important role in the transition 
process. This result was not seen in the experiments performed by White \& Saric (2000), but has become a new issue with these flight experiments.

\subsection{Transition Results with Variable Height and Appliqué Critically Spaced DREs}

The IR images displayed in Figures 55 and 56 show no change in transition location despite trying roughness elements with heights 2.5 times greater than the applique DREs. It is apparent that the cross-sectional shape of the DREs plays an important role in the transition process. With the appliqué DREs, transition moved forward with the $2.3 \mathrm{~mm}$ diameter DREs at $k=12 \mu \mathrm{m}$ and with the $1.1 \mathrm{~mm}$ diameter DREs transition moved forward at 24 to $30 \mu \mathrm{m}$. With the variable-height DREs, no change in transition is observed up to $75 \mu \mathrm{m}$ for positive-height mounds, and as low as $60 \mu \mathrm{m}$ for negative-height dimples. As a result, an attempt was made to combine the two systems by placing an appliqué DRE on top of a variable-height DRE. With this method, the applique DRE provided the critical shape while the height variation came from the pneumatic system. $1.1 \mathrm{~mm}$ DREs were concentrically placed on the $2 \mathrm{~mm}$ variableheight DREs. Utilizing this setup, there was an attempt to raise and lower the appliqué DREs above and below the critical height leading to early transition. Figure 59 shows a scan of four different DRE types with the Mitutoyo roughness tester. Figure 59 was recorded with zero differential pressure applied to the DRE insert. The force of the stylus is apparent in this figure where deflection of the tape is observed. 


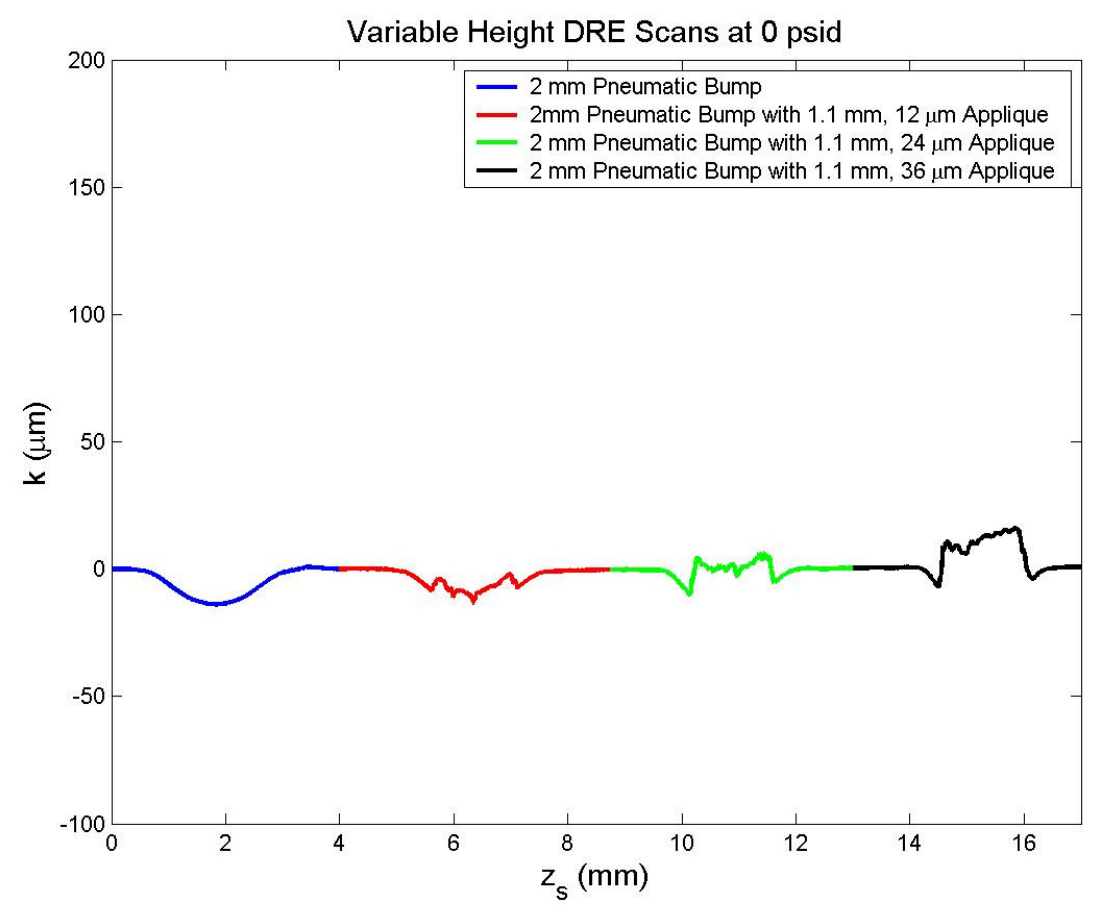

Figure 59: Variable-height DRE and appliqué DRE scans at 0 psid applied pressure.

Figures 60 and 61 show DRE scans at 2 psid and -2 psid applied pressure, respectively. It is interesting to note that under both pressure values the appliqué DREs form to the shape of the Gaussian bump and dimple. The appliqué DREs have an inklike consistency and form to the shape of the DRE. However, in both figures a defined edge is displayed at the edge of the appliqué DRE. 


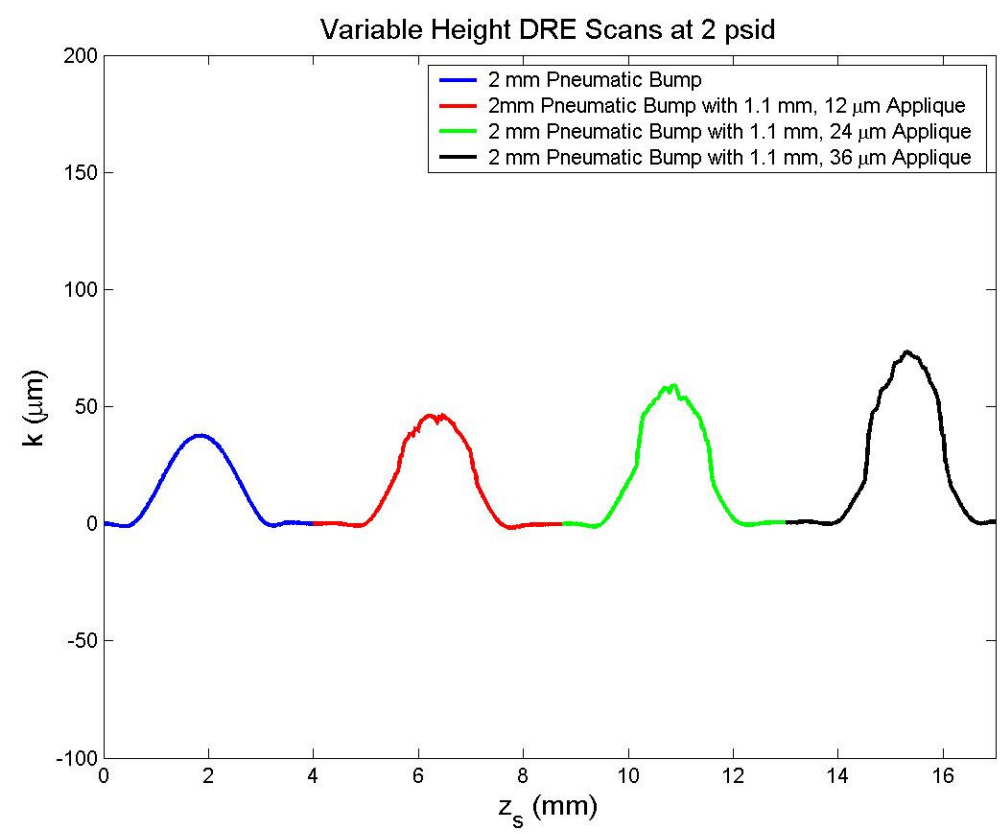

Figure 60: Variable-height DRE and appliqué DRE scans at 2 psid applied pressure.

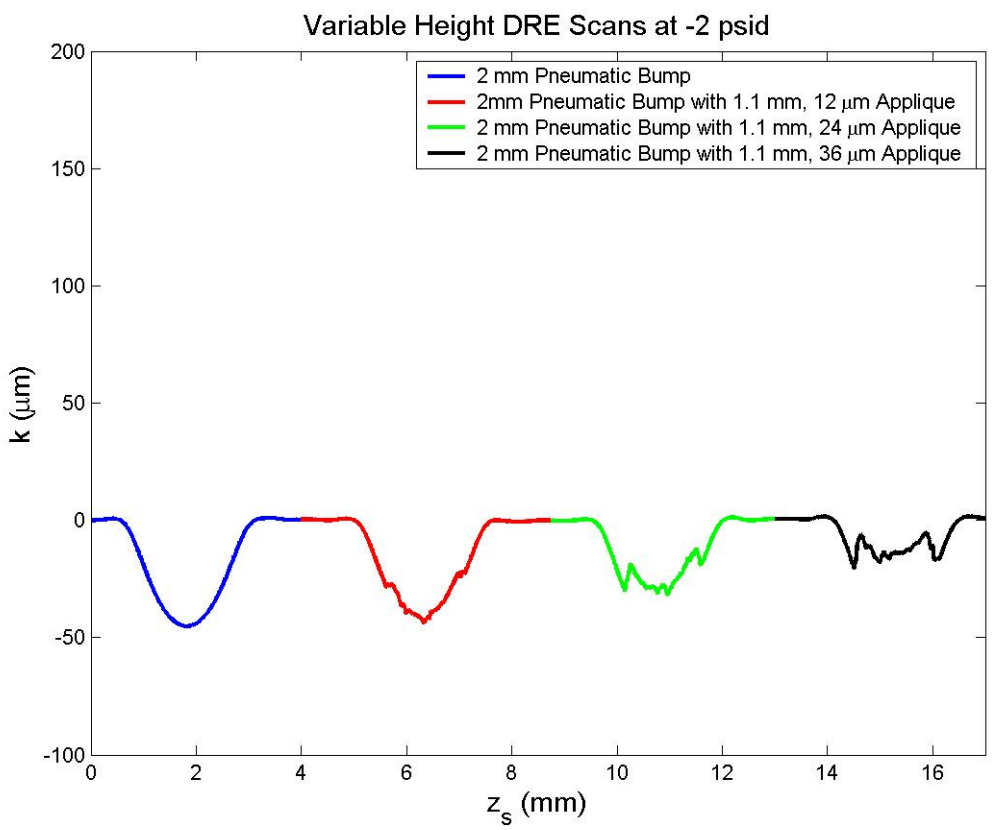

Figure 61: Variable-height DRE and appliqué DRE scans at -2 psid applied pressure. 
$1.1 \mathrm{~mm}$ appliqué DREs were applied concentrically to the $2 \mathrm{~mm}$ variable-height DREs. First $6 \mu \mathrm{m}$ appliqué DREs were applied. Figure 62 shows an IR image with zero pressure applied to the insert, therefore the roughness height was only $k=6 \mu \mathrm{m}$. For this case transition did not change; it remained at $80 \% x / c$. In Figure 62, the region where the applique DREs were applied is indicated on the IR image. During the same dive, the DRE insert was pressurized resulting in a total roughness height of $83 \mu \mathrm{m}$. Again, transition did not move from the $80 \% x / c$ location. Applique DREs were then sequentially applied until $k=18 \mu \mathrm{m}$ for one region, and $k=24 \mu \mathrm{m}$ for another region. The $24 \mu \mathrm{m}$ region was turbulent forward of $15 \% x / c$. On the other hand, the $18 \mu \mathrm{m}$ region remained laminar despite pressurizing the DRE insert up to $65 \mu \mathrm{m}$ for a total roughness height of $83 \mu \mathrm{m}$.
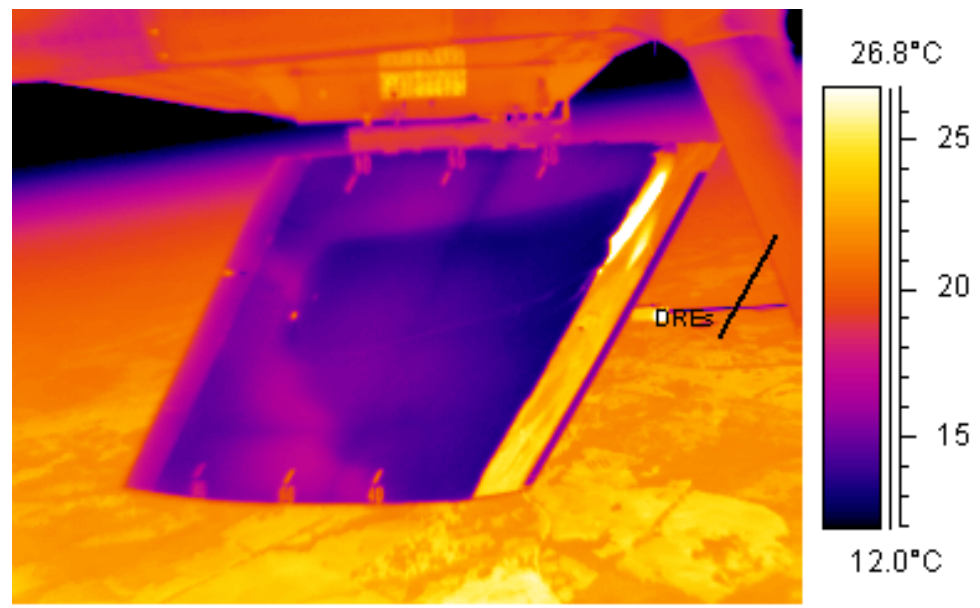

$$
\begin{array}{ll}
\text { AoA }=-2.73 \text { deg } & \text { Flight No: 080208-1 } \\
\text { Re }_{c}=7.15 \text { million } & \text { Time: 09:39:44 }
\end{array}
$$

Figure 62: $k=6 \mu \mathrm{m}\left(\right.$ Root $R e_{k}=0.3$, Tip $\left.R e_{k}=0.3, k / \delta_{\text {root }}=0.20, k / \delta_{\text {tip }}=0.20\right), d=1.1$ $\mathrm{mm}$ appliqué DREs on $2 \mathrm{~mm}$ variable-height DREs at 0 psid. 


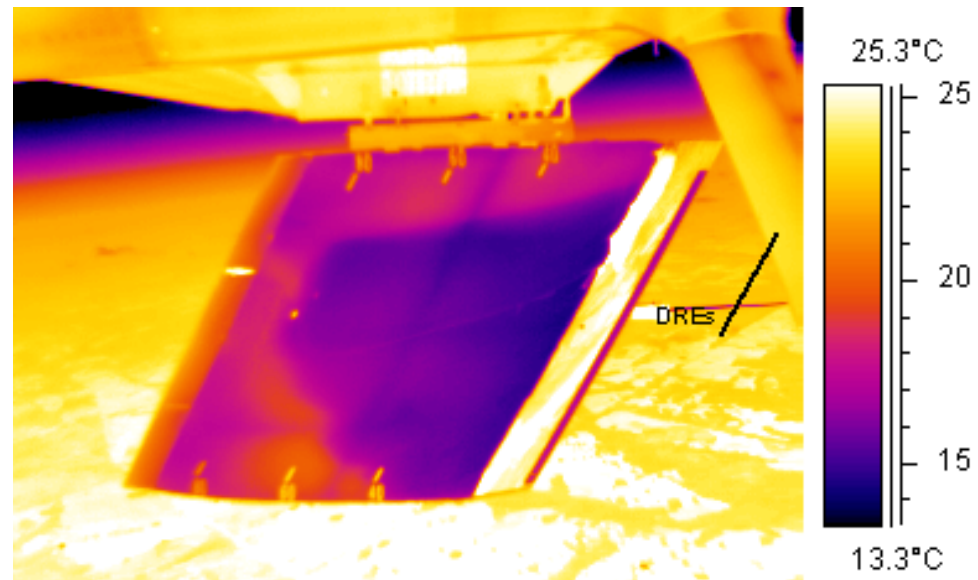

$$
\begin{array}{ll}
\text { AoA }=-2.43 \mathrm{deg} & \text { Flight No: 080208-1 } \\
\text { Re }_{\mathbf{c}}=7.07 \text { million } & \text { Time: 09:40:36 }
\end{array}
$$

Figure 63: $k=6 \mu \mathrm{m}, d=1.1 \mathrm{~mm}$ appliqué DREs on $2 \mathrm{~mm}$ variable-height DREs with the variable-height DREs at $77 \mu \mathrm{m}$. Total roughness height $=83 \mu \mathrm{m}\left(\operatorname{Root} R e_{k}=115\right.$, Tip

$$
R e_{k}=135, k / \delta_{\text {root }}=0.26, k / \delta_{\text {tip }}=0.27 \text { ). }
$$

The results with both the appliqué DREs and the variable-height DREs show that the shape of the right-circular cylinder is critical for introducing streamwise vorticity into the boundary layer. The thickness of the appliqué DRE appears to be the most important factor, and not an absolute height above or below the surface. With these results it was necessary to make further measurements to understand the disturbance created by these two different types of DREs. Section 7 discusses receptivity measurements with a calibrated hotfilm placed downstream of the different type of DREs. However, the dramatic loss of laminar flow has been described before in experiments investigating 3-D roughness near the leading edge. A comparison is made between these results and past experiments dealing with 3-D roughness. 


\subsection{Comparison with Historical Roughness Tests}

The two different roughness types tested here demonstrated strikingly different results. The transition location with the appliqué DREs could not be identified exactly, but transition was undoubtedly close to the roughness elements. Furthermore, there were no early indications that the boundary layer was about to go turbulent. An increase of 6 $\mu \mathrm{m}$ in the roughness height was enough to move transition from $80 \% x / c$ to somewhere forward of $15 \% x / c$. This behavior is similar to the experiments presented in von Doenhoff \& Braslow (1961), where no indications of disturbances are detected below a critical height for 3-D roughness applied at the leading edge. Above the critical roughness height, transition moves substantially up to the roughness element. The experiments presented in this historical paper all involve transition in 2-D boundary layers. Conversely, Radeztsky et al. (1993a) showed substantially different results with isolated 3-D roughness applied to a crossflow-dominated swept wing. In these experiments the effect of the roughness height was observed as transition gradually moved closer to the isolated roughness element. Based on these observations, the SWIFT model must generate little crossflow at this lower $A o A$ of -2.61 degrees because the behavior observed here was similar to the 2-D boundary layers described in the von Doenhoff \& Braslow (1961) paper. The IR image presented in Figure 45 supports this hypothesis. Transition is observed aft of the pressure minimum, along a uniform front, both indications of very little crossflow growth. Moreover, the transition Reynolds number plot in Figure 39 also supports this premise, where the $A o A$ at -2.61 degrees is insensitive to leading edge roughness, another indication of little crossflow growth. 
Despite the clues of a tripped boundary layer, the critical $R e_{k}$ values leading to an early transition, $R e_{k, t}$ provided in von Doenhoff \& Braslow (1961) were still substantially higher than the $R e_{k}$ values calculated here. Considering Figure 50, with [30|4.5|1.1] appliqué roughness, the root $R e_{k}$ is calculated to be 14.1 and the tip $R e_{k}$ is calculated to be 16.3. The data in von Doenhoff \& Braslow (1961) show a critical $R e_{k}$ for this roughness to be approximately 144 , based on a $d / k$ ratio of 37 . Thus the roughness heights tested here were subcritical. However, if the data presented in Carmichael (1958) are considered, there is a decrease in the $R e_{k, t}$ for spanwise-periodic cylindrical roughness elements. Carmichael (1958) showed that $R e_{k, t}$ for cylindrical roughness elements decreased as the spacing between two elements is reduced. At the most extreme case where two roughness elements are touching each other, the $\operatorname{Re}_{k, t}$ for the pair is $40 \%$ of the isolated roughness $R e_{k, t}$. Figure 64 shows the relationship observed by Carmichael (1958) for two cylindrical roughness elements placed next to each other. For the [30|4.5|1.1] appliqué roughness, the $R e_{k, t}$ is not reduced. However, the [18|4.5|2.3] appliqué roughness has a roughness spacing, $l$, to roughness diameter ratio, $l / d$, equal to 0.95, resulting in a reduction in $R e_{k, t}$ of $30 \%$. Thus, the data in Carmichael (1958) provide a possible explanation for why the larger diameter appliqué roughness elements tripped the boundary layer at a lower height than the roughness elements with the smaller diameters. It could also explain why the isolated roughness elements in Figure 49 did nothing to modify transition despite having larger roughness heights than the periodic DREs. 


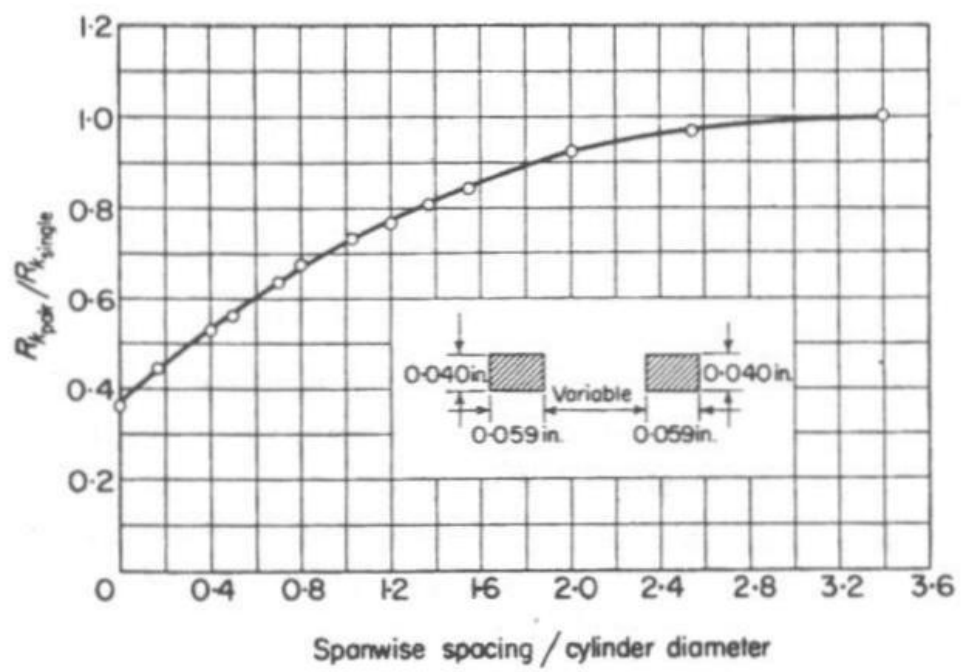

Figure 64: Plot showing reduction in $R e_{k, t}$ as two cylindrical roughness elements move close to each other. Figure from Carmichael (1958).

Despite predicting a higher sensitivity to cylindrical roughness elements placed close to each other, the $R e_{k}$ values are still lower than those required to trip the boundary layer according to the literature. Therefore, it can be assumed that the combination of roughness height, reduced spanwise spacing, and ratio of $d / \lambda_{\text {crit }}$ optimized to excite the most unstable wavelength produces a slightly different behavior. The dramatic loss of laminar flow demonstrated here is similar to the results in von Doenhoff \& Braslow (1961), but there are still some similarities to the Radeztsky et al. (1993a) experiments with sensitivity to roughness below the calculated $R e_{k, t}$. One premise is that the $d / \lambda_{\text {crit }}$ ratios tested here were already shown to be the most effective at exciting the unstable 4.5 mm wavelength. Thus, these experiments demonstrate sensitivity with subcritical roughness because the elements are optimized for exciting the most unstable crossflow 
wavelength. There is a coupling between the two different problems resulting in boundary layer trips at subcritical roughness heights.

Despite performing all the critical requirements to excite early transition, no change in transition was observed with the variable-height DREs. The roughness heights were increased well beyond those tested with the appliqué DREs. The hole diameters were increased in order to match $d / \lambda_{\text {crit }}$ ratios shown to excite the crossflow instability. And lastly, the larger diameters tested also decreased the spacing in between the roughness elements, which should have reduced the $R e_{k, t}$ based on Figure 64 . Nevertheless, transition did not move with the variable-height DREs. Therefore, it appears that the experiments of Carmichael (1958) are only applicable to cylindrical roughness elements and not the bumps and dimples tested here. The $R e_{k}$ values tested with the variable-height DREs were just below the critical values given in von Doenhoff and Braslow (1961), explaining why early transition was not observed. In light of these findings, it is necessary to understand the receptivity problem to leading- edge roughness. Only so much can be learned by observing changes to transition with an IR camera. Thus, the next step in the program was to make receptivity measurements in order to better understand the effect of roughness shape and height on the transition process. 


\section{RECEPTIVITY MEASUREMENTS}

The multi-element hotfilm array is a valuable tool for transition location, shock detection, and crossflow vortex spacing identification. In many applications the small size of the sensors and small thickness of the substrate do not trip the laminar boundary layer. Furthermore, hotfilm sensors have time responses in the $\mathrm{kHz}$ range so high frequency signals can be analyzed. With these features, multi-element hotfilm arrays have been widely used in both wind tunnel and flight test experiments.

Transition can be identified with a hotfilm by looking at the spectral content of the voltages acquired from a constant temperature, constant current, or constant voltage anemometer. An even easier approach is to plot voltages from a hotfilm on an oscilloscope and observe the jump in RMS voltage across the transition front. These same procedures can also be used for identifying shocks (Moes et al. 1997). Moreover, both amplitude and phase information can be used for identifying crossflow vortex spacing on a swept-wing (Magalan et al. 1990, Agarwal et al. 1992). However, in each of these aforementioned experiments the actual shear stress values are not calculated, only voltages are recorded. In order to resolve the importance of roughness shape and height, a calibrated hotfilm was placed downstream of modulating DREs to measure varying disturbance amplitudes. These measurements can then be compared with the NPSE calculations in Figure 44. Uncalibrated hotfilms could not achieve these results due to variations in sensor resistances between each channel. It is possible that an uncalibrated hotfilm could measure the crossflow vortex spacing as was done in the 
Magalan et al. (1990) and Agarwal et al. (1992) experiments, but to measure the disturbance amplitude requires a calibrated hotfilm array. Therefore, a calibration scheme was needed for calibrating the hotfilms in flight. A Preston tube was selected as the known shear stress measuring device needed for the calibration procedure.

\subsection{Hotfilm Array Design}

A custom hotfilm was designed for measuring the crossflow amplitude and spacing on the SWIFT model. With a maximum crossflow spacing of $4.5 \mathrm{~mm}$, a multielement hotfilm array was needed for measuring the high and low shear stress regions in between areas of strong crossflow growth. The spacing of each hotfilm sensor needed to be small enough to resolve the shape of the crossflow wavelength, in addition to having a long enough span to capture at least one period. The $4.5 \mathrm{~mm}$ crossflow spacing is measured parallel to the leading edge. When each hotfilm sensor is placed perpendicular to the inviscid streamline, the crossflow spacing at the hotfilm is approximately $3.9 \mathrm{~mm}$, resulting in an even finer spacing required for the hotfilm design.

The Cessna O-2A aircraft could only carry an additional 100 lbs of instrumentation, at the expense of performing one dive per flight. Flights prior to these receptivity measurements always had enough fuel to perform two dives per flight. The FRL has 30 channels of constant temperature anemometers (CTAs) divided between three chassis. Each chassis weighs $50 \mathrm{lbs}$ so only two chassis could be installed in the 
aircraft. Therefore, the goals set for measuring the crossflow vortices needed to be met with a maximum of 20 channels.

The multi-element hotfilm used in these experiments was constructed by Tao Systems. The design utilized the smallest sensors and lead widths that the manufacturer had confidence in constructing. Sensors with a 0.002" $(50 \mu \mathrm{m})$ diameter and a length of $0.02 "(0.51 \mathrm{~mm})$ were used with the smallest lead widths available. Figure 65 shows the details of the hotfilm sensor designed for these experiments. The smallest dimensions available for the design resulted in a staggered hotfilm where the line intersecting the center of each sensor was swept back 42 degrees. If this line where swept back at 30 degrees, then the sweep of each sensor would have matched the $\Lambda_{L E}$, and each sensor would be located at the same $x / c$ location on the model. However, the limitations in manufacturing tolerances forced the sensors to be along a 42 degree swept line resulting in a change in $x / c$ location of $0.2 \%$ for 20 channels. The change in disturbance growth over $0.2 \% x / c$ is calculated to be negligible, therefore the change in $x / c$ location for the hotfilm is assumed to be zero. 


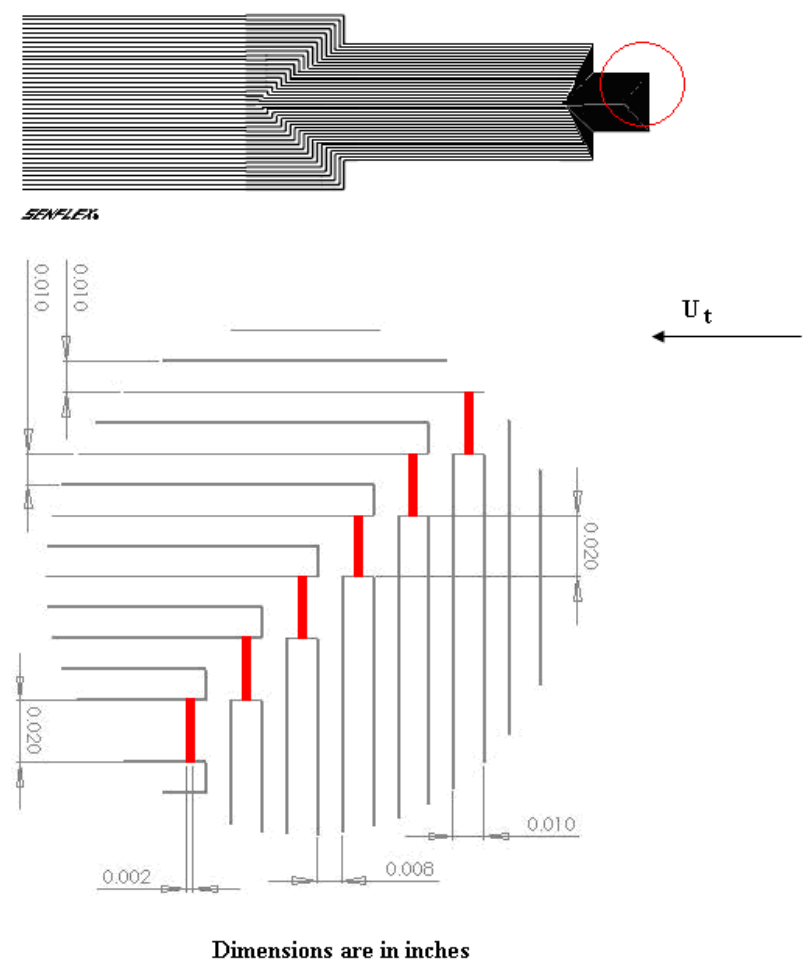

Figure 65: Custom hotfilm design for measuring disturbance amplitudes and crossflow vortex spacing.

It was desired to use the $2 \mathrm{~mm}$ diameter, variable-height DREs centered on the root pressure port row for the hotfilm calibration and receptivity measurements. The larger-diameter, variable-height DREs were more effective at introducing a disturbance than the smaller $1.1 \mathrm{~mm}$ diameter variable-height DREs located lower on the DRE insert. Therefore, the hotfilm would be mounted somewhere along the root pressure port row. Despite showing zero change in transition location with the variable-height DREs, an active control system was needed in order to turn off the DREs for hotfilm calibration. A uniform shear stress was needed across the hotfilm to calibrate each sensor. This constraint was met by calibrating at a stable $A o A$ demonstrating little 
crossflow growth, (-2.61 degrees $A o A)$ with the critically-spaced DREs turned off. This idea was adopted from the calibration suggestions made by Chapman (1996). There is no way of "turning off" the appliqué DREs, therefore variable-height DREs were needed for the hotfilm calibration.

Provided the boundary layer is still laminar, the Branch II point, or location of highest disturbance growth, is the preferred location for the hotfilm. However, since the $2 \mathrm{~mm}$ diameter DREs were only drilled along a 12-inch span centered on the root pressure port row, there was a limit on how far aft the hotfilm could be mounted. Figure 66 shows the hotfilm located at $34 \% x / c$, which proved to be the farthest aft mounting location for the hotfilm based on a 12-degree half-angle wedge generated by the first and last DRE, as suggested by Saric (2007). Furthermore, the model is still laminar at $34 \%$ $x / c$ at the two AoAs of interest, which is a necessary requirement for measuring the crossflow vortices.

The spacing between the surface-mounted thermocouple, static pressure port, Preston tube, and first hotfilm channel were all $0.9 "$ along a line of constant $x / c$. Prior to mounting the hotfilm, which is a pseudo-permanent attachment, a wind tunnel test was conducted to see how close each of these components could be displaced from each other. At the tunnel's maximum speed of $40 \mathrm{~m} / \mathrm{s}$, it was found that the Preston tube could be displaced 0.5 inches from the static pressure port. Similarly, the Preston tube had to be 0.8 inches from the first hotfilm channel before the shear stress measurement 
was affected by the presence of the Preston tube. The 0.9 inches was selected as the appropriate distance in order to fair in the edges of the hotfilm and surface-mounted thermocouple with Bondo to avoid any forward facing steps. Any closer and it would have been difficult to sand down the faired in edges of the hotfilm and surface-mounted thermocouple. The spacing between each component is assumed to be small enough that each component experiences the same shear stress, provided the crossflow growth is minimal during the calibration.
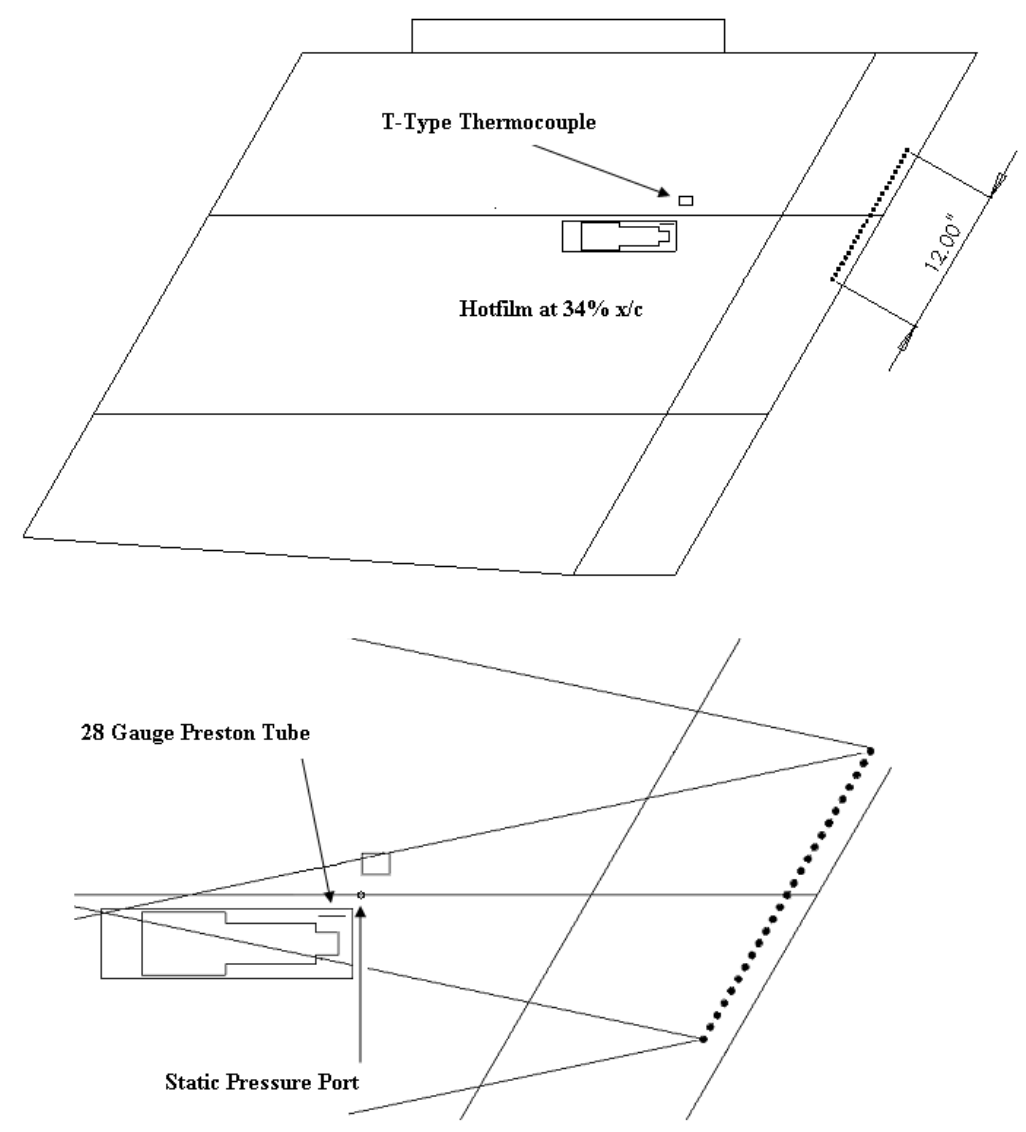

Figure 66: Schematic of hotfilm, Preston tube, surface-mounted thermocouple, and static pressure port on the SWIFT model. 


\subsection{Preston Tube}

The flight experiments here are subject to large temperature excursions during the dive profile. As a result, a comparison of raw voltages from each sensor would be meaningless. A Preston tube was placed next to the hotfilm to provide the known measure of shear stress for the calibration scheme. Preston (1954) first developed the procedure for using a surface-mounted Pitot-tube for measuring shear stress at the surface of external and internal flows. Patel (1965) re-addressed the calibration from Preston's original paper and has developed the most widely used set of equations for measuring shear stress with a Preston tube. Patel also addressed the problem of operating a Preston tube in pressure gradients, where the accuracy of the Preston tube decreases in favorable pressure gradients as compared to adverse pressure gradients.

Most of the literature deals with Preston tubes mounted in either external or internal turbulent boundary layers. However, Bechert (1995) provides an asymptotic equation which combines the three pressure ranges covered by Patel (1965), in addition to providing an equation for use of a Preston tube in laminar flow, which is used here. Bechert's equation in laminar flow proved to be the most accurate based on a comparison between experimental measurements of wall shear stress, $\tau_{w}$, and computations. Equations 8 and 9 were used for calculating $\tau_{w}$ in the laminar boundary layer on the SWIFT model. 


$$
\begin{gathered}
\tau^{+}=4 \sqrt{\frac{\Delta p^{+}}{3}} \\
\tau^{+}=\frac{\tau_{w} d_{\text {prest }}^{2}}{\rho v^{2}}, \Delta p^{+}=\frac{\Delta p d_{\text {prest }}^{2}}{\rho v^{2}}
\end{gathered}
$$

In Equation 8, $\Delta p$ is the differential pressure measured between the Preston tube and the nearby static pressure port. $d_{\text {Prest }}$ is the outer diameter of the Preston tube. $\rho$ and $v$ are the fluid density and kinematic viscosity of the freestream, respectively. Bechert (1995) specifies limits for $\Delta p^{+}$in which Equation 8 is valid. The $\Delta p^{+}$values recorded in these experiments range from 50 to $300 \mathrm{kPa}$, which extend to slightly outside the range specified by Bechert.

The requirements for using a Preston tube to measure $\tau_{w}$ are that the tube must sit within the viscous sub-layer of the boundary layer, with a general rule of thumb that the diameter of the Preston tube must be a small percentage of the boundary layer height. This requirement becomes even more difficult to fulfill with a laminar boundary layer, requiring an even smaller Preston tube. The Preston tube used here was a 28-gauge hypodermic stainless steel needle with a flat, round mouth. The outer diameter was $0.014 "$ which was $36 \%$ of the boundary layer height at the $34 \% x / c$ location. Smaller hypodermic needles were available, but the time response became too slow to be useable in the dive attitude where conditions were constantly changing. As a result, a 
compromise was made to find a Preston tube small enough to meet the requirements set by Patel (1965) on the percentage of boundary-layer height, but to be large enough to have a sufficient response time to provide time-accurate data in the dive. The 28 -gauge hypodermic needle was a good compromise because the time lag in this tube was small enough to be useful in the dive, but the diameter was not excessively large compared to $\delta$.

Two Honeywell Sensotech FP2000 pressure transducers were mounted inside the internal cavity of the SWIFT model to reduce the tube length to the transducers. Furthermore, the 28-gauge Preston tube was only one inch long. The Preston tube was quickly stepped up to a two-inch long, 0.040" outer diameter, stainless-steel tube. This was followed by six inches of 0.040 " inner diameter polyurethane tubing. Lastly, a fivefoot section of 0.063 " inner diameter polyurethane tubing led to the pressure transducers inside the model. Furthermore, the tubing for the static pressure port was purposely extended to match the time lag of the smaller-diameter Preston tube. In addition to the relatively large diameter of the Preston tube used here, there is a question regarding the accuracy of the Preston tube in the favorable pressure gradient tested here. However, the goal of the current experiments is to find a difference in shear stress between the peaks and valleys generated by the crossflow vortices. Therefore, any error in the absolute calibration of the hotfilm is subtracted out when taking the difference. With this overall goal in mind, the decrease in accuracy with the relatively large Preston tube and use in a favorable pressure gradient was acceptable. 


\subsection{Temperature Compensation}

Prior to flight, several measurements of the voltage output of each hotfilm channel were made. These measurements were made in the FRL's un-insulated hangar, subject to temperature changes as much as $2{ }^{\circ} \mathrm{C} /$ hour. Each channel on the hotfilm array was adjusted with a gain of 10 and an overheat ratio of 1.2 to provide sufficient sensitivity during flight. With this gain, the zero flow voltage, $E_{o}$, of each channel would change by approximately $-0.45 \mathrm{~V} /{ }^{\circ} \mathrm{C}$, as depicted in Figure 67 . Note that in all of the following figures displaying voltages from the CTAs, an offset of 5 volts was added to each channel. The analog input channels on the DAQboards used in these experiments are all bipolar. In order to get the highest resolution out of these 12-bit DAQboards, the DC offset of each channel has been set to an average of 0 volts. To avoid sign changes after acquisition, an offset of 5 volts was added to each channel. 


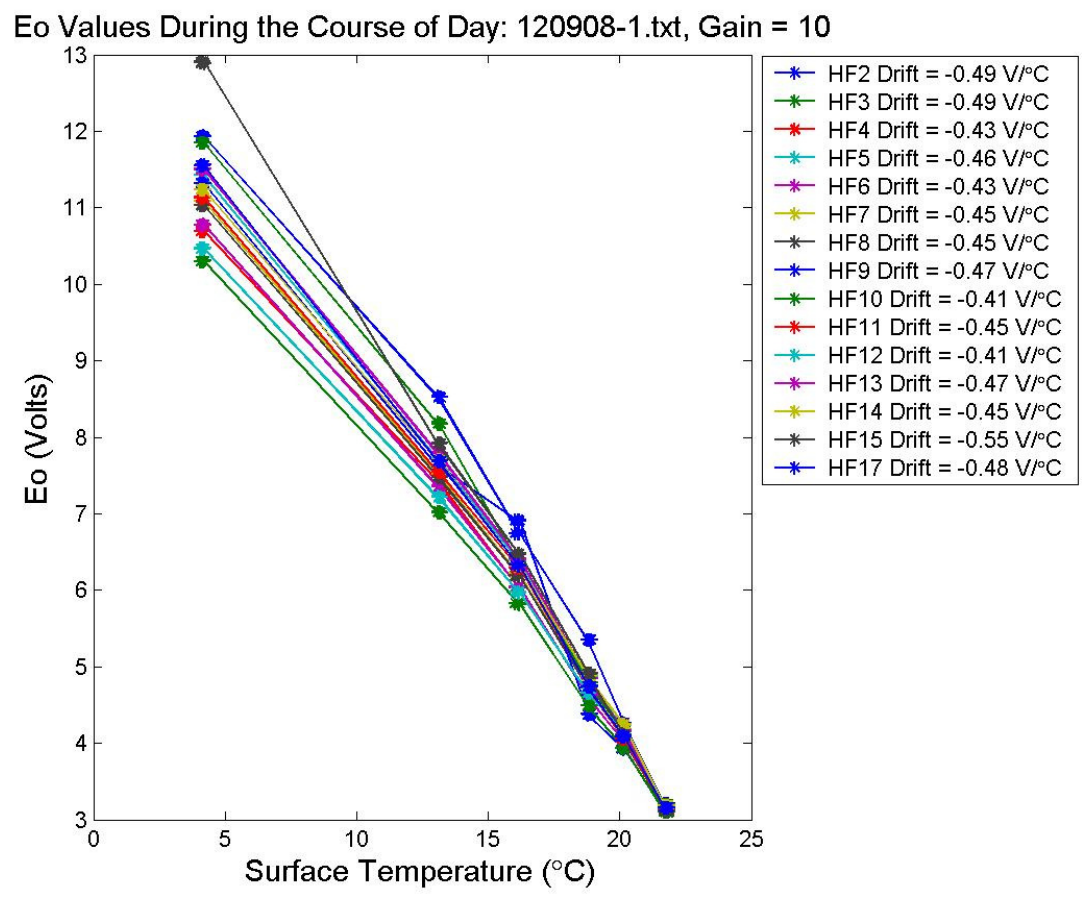

Figure 67: $E_{o}$ measurements recorded in the FRL hangar.

The temperature drifts displayed in Figure 67 agree with the measurements made by Radeztsky et al. (1993b), where they observed temperature drift with CTAs and hotwires and found a linear relationship between the voltage and temperature. Based on the findings in the hangar, prior to flight, it was obvious that temperature compensation would be the most important problem to address in order to resolve any useful data. Furthermore, the temperature drifts reported in Radeztsky et al. (1993b) were recorded at constant velocity as the un-cooled tunnel heated up. This constant velocity was not feasible because the aircraft would run out of fuel before a large temperature difference, at constant attitude, could be achieved in flight. So, the temperature drift had to be measured on the ground with zero air flow over each channel. To prevent sensor 
burnout, each hotfilm channel was disconnected from the CTA circuit in between each recording, which were typically spaced one hour apart.

Preston tube data were recorded during three phases of each flight. During the cold soak, while the crew was waiting for the model to cool down uniformly, the pilot increased the airspeed at five-knot increments holding constant $A o A$, while the FTE recorded data. Once the model was at a uniformly cold temperature, the dive was initiated, again at a constant $A o A$. The FTE recorded data continuously during the dive at $20 \mathrm{~Hz}$. At the end of the dive the pilot decelerated at constant AoA, while the FTE continued to record the slow-down period. During the return to base, the pilot performed another calibration run, but this time the temperature was higher at the lower altitude. Figure 68 shows the Preston tube $\tau_{w}$ plotted against the true airspeed recorded with the five-hole probe. In Figure 68, data points outside the targeted $\beta$ range of $2.61 \pm 0.10$ degrees have been deleted from the plot. Additionally, Figure 69 shows the calibration curve needed to correlate $\tau_{w}$ measured from the Preston tube and each of the 17 hotfilm voltages, $E$, recorded during the dive. 


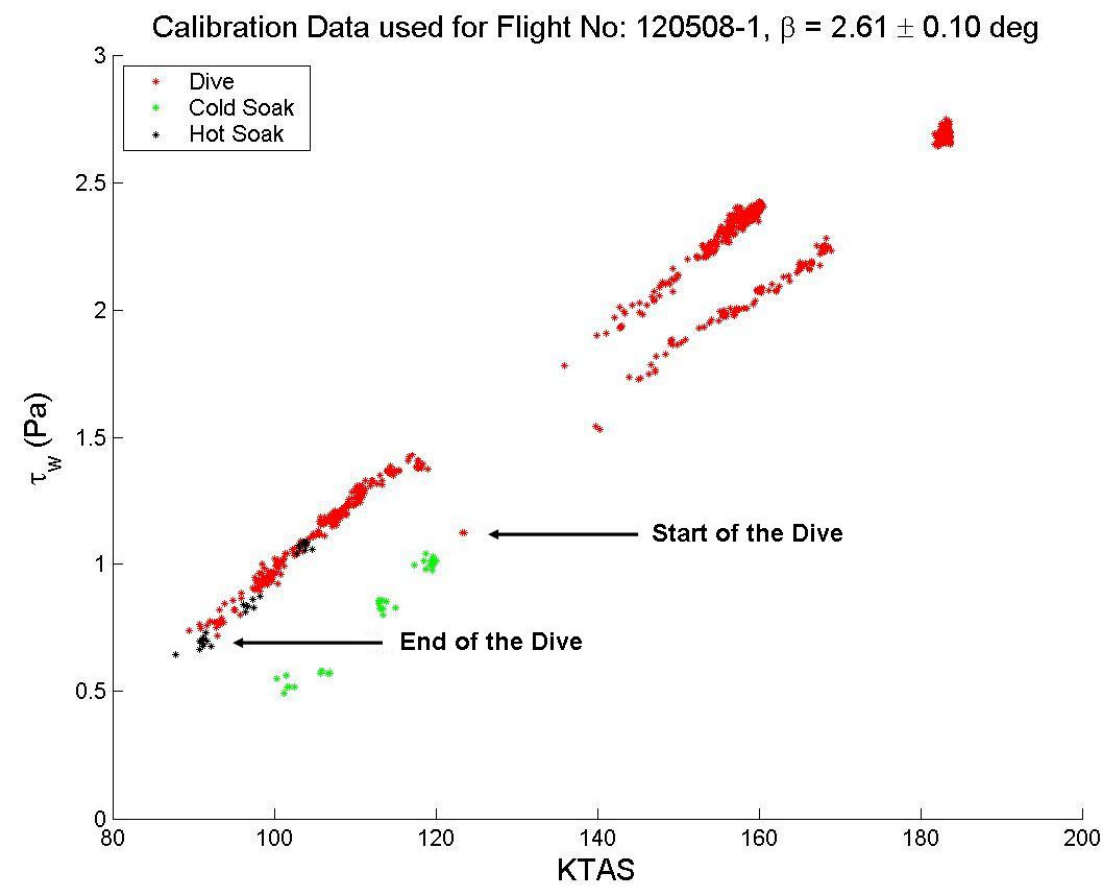

Figure 68: Filtered Preston tube data during the cold soak, dive, and hot soak.

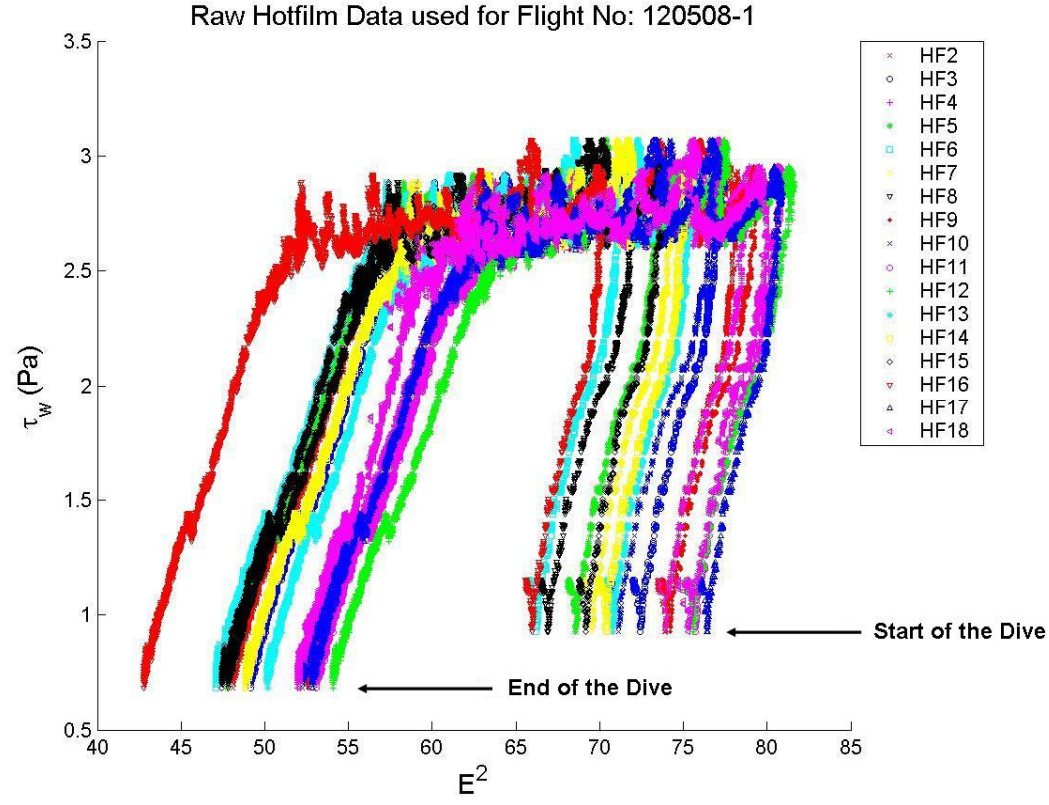

Figure 69: Wall shear stress and recorded hotfilm voltages during the dive. 
In both Figures 68 and 69, there are obvious hysteresis loops which need to be corrected before a calibration can be attempted. In Figure $68, \tau_{w}$ is higher at the bottom of the dive because the dynamic viscosity is higher at the warmer temperatures at low altitude. In Figure 69, the hotfilm voltages are lower at the bottom of the dive because less voltage is required at the warmer temperatures to maintain the overheat ratio in each hotfilm channel. In light of Figures 68 and 69, a temperature compensation scheme was required to generate a calibration curve that was independent of temperature.

Radeztsky et al. (1993b) suggest that the temperature drifts recorded for each hotfilm channel can be used to correct the measured voltages to a compensated voltage based on a standard temperature. Equation 10 has been proposed here to calculate a temperature compensated voltage for each hotfilm channel.

$$
E_{\text {comp }}=E_{\text {measured }}+m\left(T_{\text {nom }}-T_{s}\right)
$$

In Equation $10, E_{\text {measured }}$, is the recorded voltage subject to temperature changes and $m$ is the temperature drift for each hotfilm channel with each initial value displayed in Figure 67. $T_{n o m}$ is a nominal temperature to which all voltages are corrected. In these experiments, the ground temperature during takeoff was used. $T_{s}$ is the surface temperature of the model next to the hotfilm. The surface temperature was selected as the more appropriate temperature to use because shadows and solar radiation from direct sunlight affect the measured voltage of each hotfilm channel. Since the surface-mounted 
thermocouple is mounted next to the hotfilm, the surface-mounted thermocouple experiences the same solar radiation and be more representative of the actual temperature experienced by each hotfilm sensor.

After plugging in the temperature drift values recorded in Figure 67 for each individual channel and re-plotting in the manner of Figure 69, the calibration curves still did not collapse on top of each other. $m$ was then adjusted for each channel until the calibration curve for the top and bottom of the dive eventually collapsed on top of each other into one curve, indicating a temperature invariant calibration curve. After doing this for each channel, the temperature drift for 17 hotfilm channels averaged to $m=-0.28$ $\mathrm{V} /{ }^{\circ} \mathrm{C}$. This result compares with the average temperature drift recorded in the hangar of $\mathrm{m}=-0.45 \mathrm{~V} /{ }^{\circ} \mathrm{C}$. The in situ adjustment of $m$ for each flight was expected because the heat conduction loss to the model is different for each flight. Therefore, it cannot be expected that the temperature drift recorded on the ground, in the hangar, would be universally applicable to all flights. This empirical observation is in agreement with Sarma \& Moes (2005), where heat conduction to the substrate is the principal problem in calibrating surface-mounted hotfilms, and must be accounted for on each model for every test. Once each temperature drift value is finalized for each flight, a calibration curve (like the one depicted in Figure 70) is generated, that is now temperature invariant. 


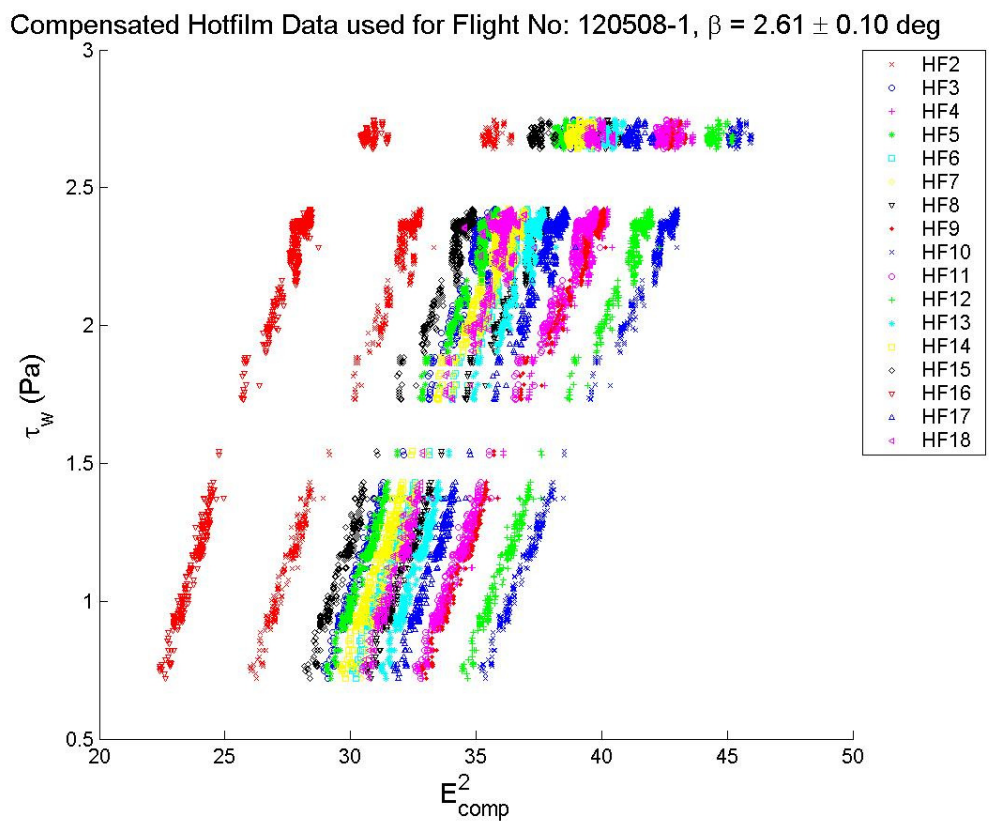

Figure 70: Example of finalized calibration curves after applying temperature compensation.

\subsection{Hotfilm Calibration}

Bellhouse \& Schultz (1966) were the first to address the relationship between the voltage output of a CTA and the $\tau_{w}$. Equation 11 shows the relationship that was derived between the two variables of interest.

$$
E_{\text {comp }}{ }^{2}=A \tau_{w}{ }^{1 / n}+E_{o}{ }^{2}
$$

Now $\tau_{w}$ is solved for as the dependent variable, 


$$
\tau_{w}=\left(\frac{E_{\text {comp }}^{2}}{A}-\frac{E_{o}^{2}}{A}\right)^{n}
$$

The constant $n$ is typically $\geq 3$. If $n$ is assumed to be 3 , then equation 12 can be expanded using the Binomial Theorem using $E_{\text {comp }}{ }^{2}$ as the independent variable calculated from Equation 10,

$$
\tau_{w}=a\left(E_{\text {comp }}^{2}\right)^{3}+b\left(E_{\text {comp }}^{2}\right)^{2}+c\left(E_{\text {comp }}^{2}\right)+d
$$

A Least Squares third-order polynomial curve fit can be applied to find the constants $a$, $b, c$, and $d$ in Equation 13 for each channel. The constants $A$ and $E_{o}$ have been absorbed into the constants $a, b, c$, and $d$. Figure 71 shows a curve fit using a third-order polynomial equation which fits nicely with the temperature-compensated data recorded during the dive. This procedure is then repeated for each hotfilm channel. 


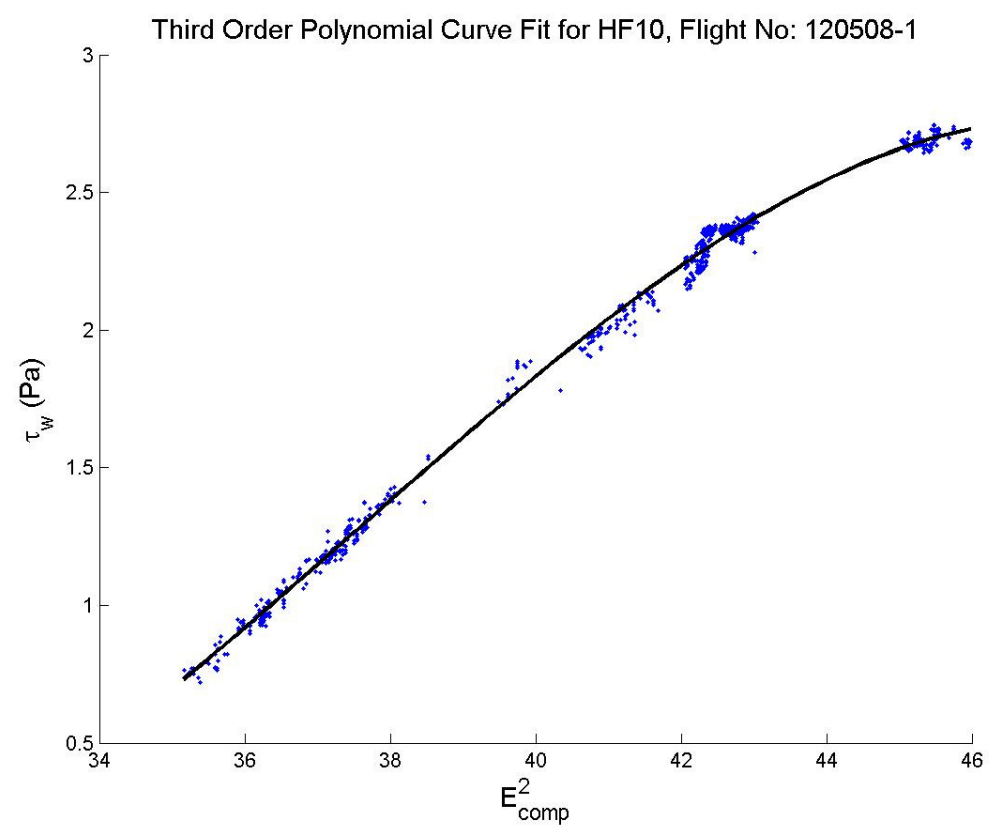

Figure 71: Example calibration curve for one of the hotfilm sensors.

Each calibration curve is subjected to two filters. The DREs were turned off, and the $A o A$ on the model was held within a \pm 0.1 degree band about the targeted $A o A$ for that flight. Using this procedure to eliminate data with strong crossflow growth with the DREs on, and bad data points away from the targeted AoA, clean calibration curves were generated for all hotfilm channels and all flights tested so far.

\subsection{Receptivity Results}

After installing the hotfilm, Preston tube, and surface-mounted thermocouple, a 20-degree lens on the IR camera was installed to get a close image of this area. The intent here was to confirm that the boundary layer over the hotfilm was still laminar after 
installation and fairing in the edges with Bondo. Figure 72 shows an IR image with the 20-degree lens confirming that the boundary layer in this area remained laminar. Figures 73 and 74 show representative IR images with the 60-degree lens for the two AoAs tested here. Figure 73 shows the hotfilm located just upstream of transition at -4.76 degrees $A o A$. Figure 74 shows laminar flow back to $80 \% x / c$ at the $A o A$ of -2.59 degrees. At this $A o A$ the hotfilm is located in a stable region with little crossflow growth. In both Figures 73 and 74 the variable-height DREs were pressurized up to $50 \mu \mathrm{m}$, but like before, no change in transition was observed with the IR camera. The transition results with the IR camera were also confirmed with Power Spectral Density (PSD) plots of one of the hotfilm channels, also confirming a laminar boundary layer. Figure 75 shows a PSD plot for hotfilm \#2 at the two AoAs of interest. Note that hotfilm \#2 is the furthest upstream sensor on the hotfilm array. Figure 75 shows two laminar PSD plots, confirming that even at the less stable $A o A$ of -4.69 degrees, the boundary layer is still laminar. 


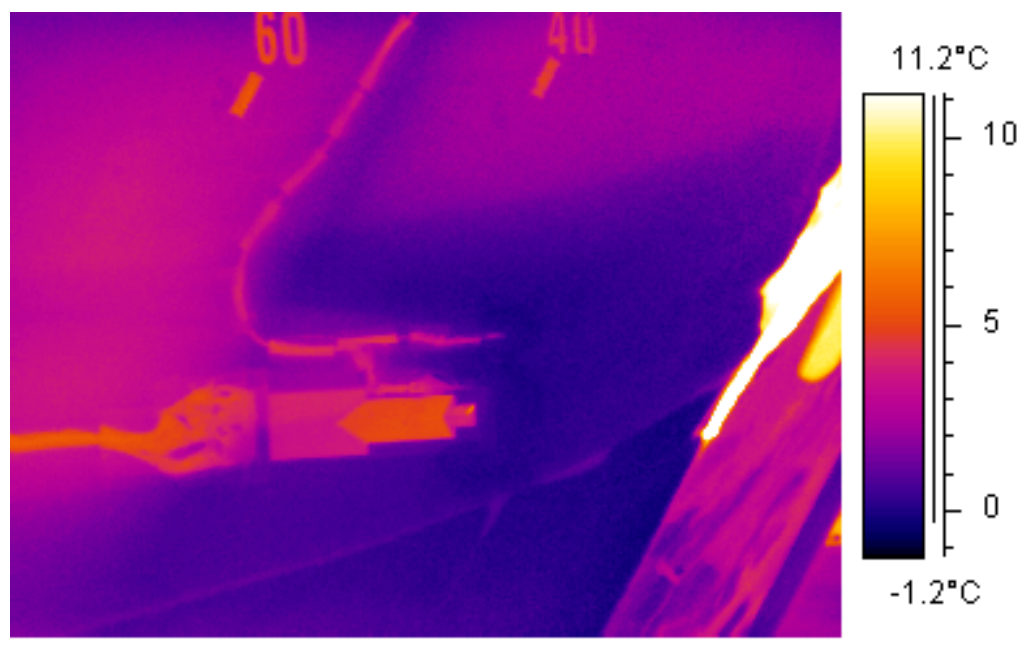

$$
\begin{array}{ll}
\mathrm{AoA}=-2.88 \mathrm{deg} & \text { Flight No: 112108-1 } \\
\operatorname{Re}_{c}=7.36 \text { million } & \text { Time: } 17: 17: 54
\end{array}
$$

Figure 72: IR image showing a laminar boundary layer over the hotfilm, Preston tube, static pressure port, and surface-mounted thermocouple.

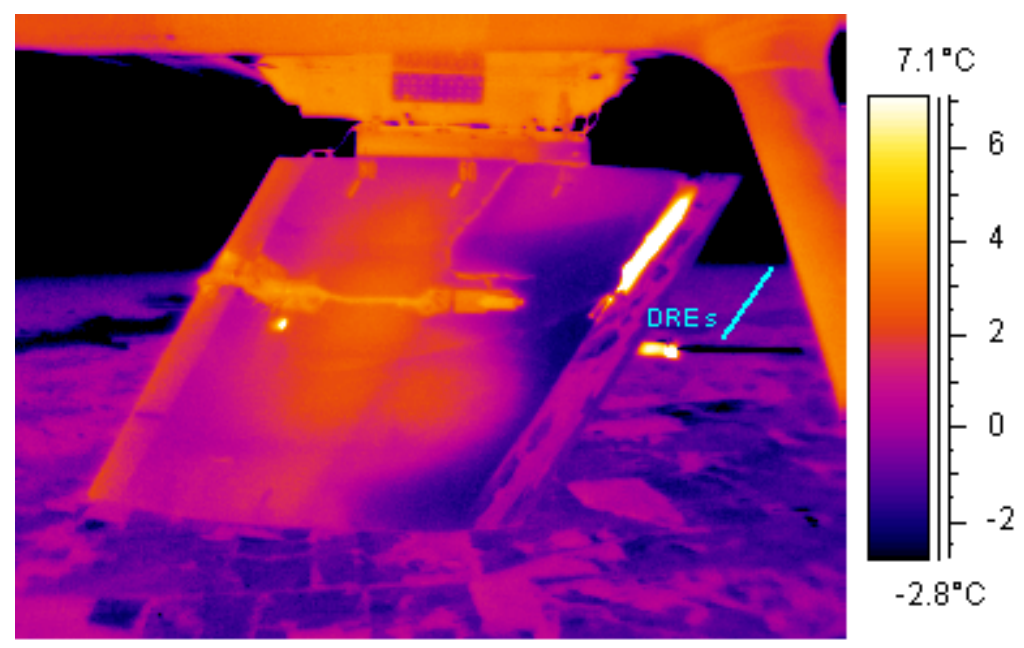

$$
\begin{array}{ll}
\mathrm{AoA}=-4.76 \mathrm{deg} & \text { Flight } \mathrm{No}_{0}: 120408-1 \\
\operatorname{Re}_{c}=7.64 \text { million } & \text { Time: 17:05:26 }
\end{array}
$$

Figure 73: IR image of the hotfilm located just upstream of transition. 


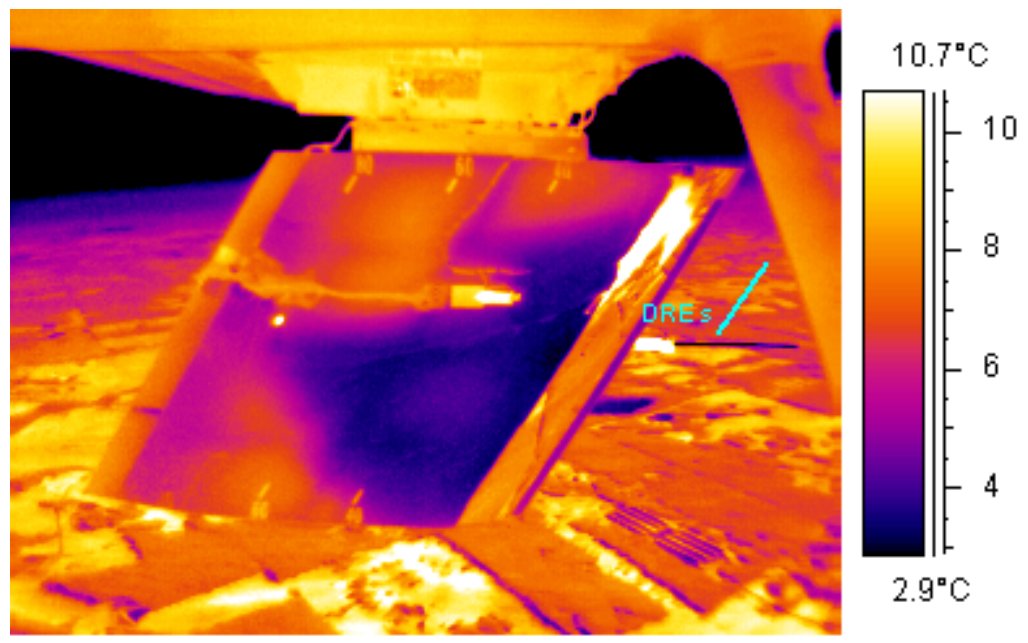

$$
\begin{array}{ll}
\mathrm{A} 0 \mathrm{~A}=-2.59 \mathrm{deg} & \text { Flight No: 120708-1 } \\
\mathrm{Re}_{\mathbf{c}}=7.61 \text { million } & \text { Time: 09:56:24 }
\end{array}
$$

Figure 74: IR image of the hotfilm located in a stable laminar boundary layer.

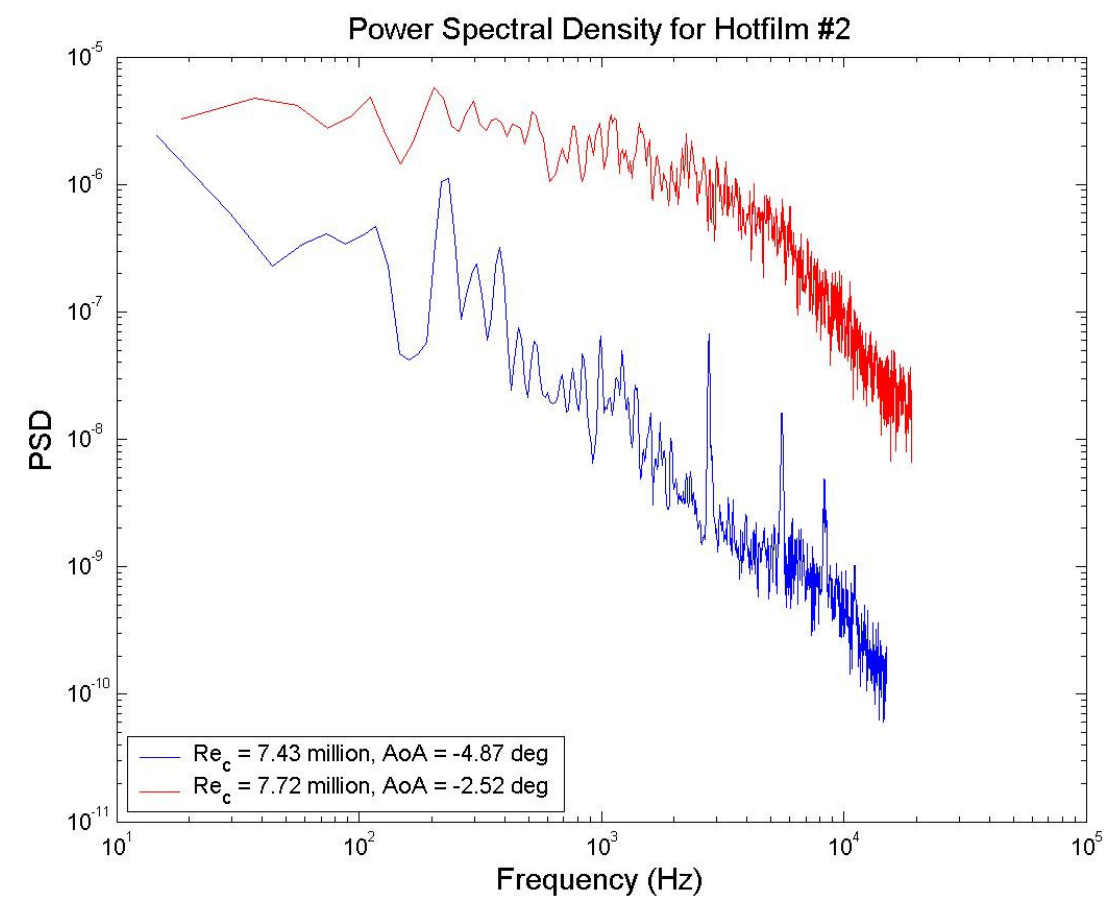

Figure 75: Power Spectral Density plot for hotfilm \#2 at two different AoAs. 
Two $A o A$ conditions have been tested thus far. -2.61 degrees $A o A$ proved to have very little crossflow growth at the $34 \% x / c$ location. Even with the variable height DREs pressurized to $50 \mu \mathrm{m}$, no crossflow structure is apparent in the hotfilm signal. Vacuum was also tested with the DREs drawn down to $-50 \mu \mathrm{m}$, but with similar results with no signs of crossflow. For this $A o A$, the hotfilm is probably mounted too close to the leading edge and the crossflow vortices have not grown enough to be recorded with the hotfilm. On the other hand, the test point at -4.69 degrees $A o A$ shows strong signs of crossflow, even without the DREs pressurized or evacuated. A definite structure spaced $4.5 \mathrm{~mm}$ is apparent in the signal. Furthermore, the signal gets stronger as the DREs are pressurized. The same is true during the evacuated flights where the DREs were drawn down to $-50 \mu \mathrm{m}$. A strong $4.5 \mathrm{~mm}$ structure was also observed. Figure 76 shows a comparison between the four flights conducted with the pneumatic DREs. 


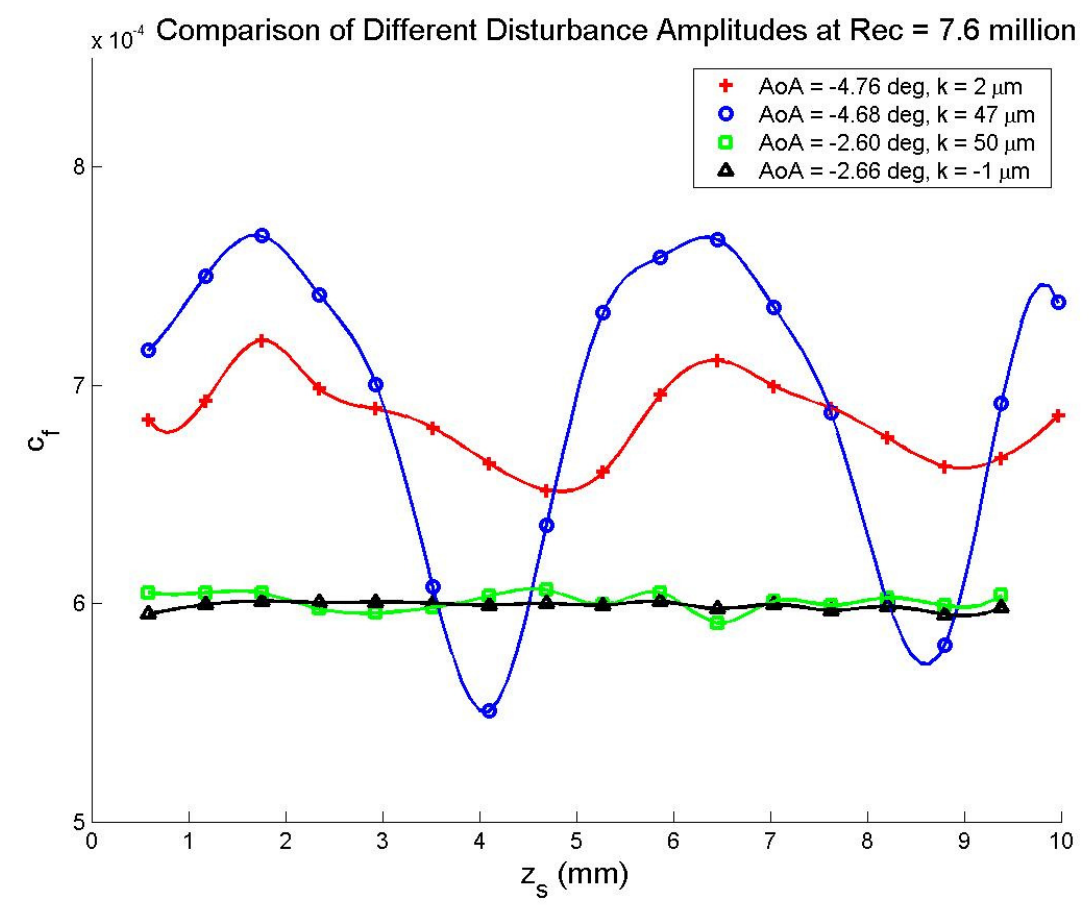

Figure 76: Comparison of different disturbance amplitudes with DREs spaced $4.5 \mathrm{~mm}$ apart.

Figure 76 shows that the hotfilm covered two crossflow vortices. However, videos created after each flight showed as much as four different crossflow vortices being captured by the hotfilm, but still at the $4.5 \mathrm{~mm}$ spacing. As $\alpha$ fluctuates during the dive, four crossflow vortices move in and out of the frame, with only two vortices captured during a given time. This small spanwise modulation of the stationary crossflow vortices explains why the heat generated by the crossflow vortices does not appear in the IR images. 
In Figure 76 , the first curve at an $A o A$ of -4.76 degrees is in phase with the second curve with the DREs pressurized. The electronic pressure regulator used in these experiments has difficulty holding zero differential pressure. There is always some residual pressure in the system on the order of 0.05 psid, resulting in DRE heights of less than $\pm 2 \mu \mathrm{m}$. This small roughness height appears to be strong enough to force a spanwise invariant structure, which is not expected for naturally occurring crossflow vortices due to random surface roughness. Naturally occurring crossflow vortices would not be in phase with vortices generated by excited roughness elements. However, all test points at the -4.69 degree $A o A$ test condition show a strong $4.5 \mathrm{~mm}$ structure that is always in phase with the DREs on and off. Thus, a comparison can now be made between disturbances generated by a $2 \mu \mathrm{m}$ bump versus a $50 \mu \mathrm{m}$ bump. It appears as though there is a non-linear relationship between the roughness height and the disturbance amplitude measured downstream of the roughness elements. The signal generated by the $2 \mu \mathrm{m}$ roughness element is close to the signal generated by the $50 \mu \mathrm{m}$ bump, despite having a much smaller height. However, the signals measured at -4.69 degrees $A o A$ are measured just upstream of transition, so the amplitudes may have already saturated as measured by Reibert et al. (1996) and calculated by Haynes \& Reed (2000). A comparison between the range of disturbance-amplitude inputs used in Figure 44 and the crossflow $C_{f}$ amplitudes measured in Figure 76 can now be made. This comparison is the last remaining link between the DREs and the NPSE calculations. Now a technique is available for performing this comparison. 


\section{SWEPT-WING LAMINAR FLOW CONTROL WITH DRES}

The polished leading edge demonstrated that large amounts of NLF were achievable at moderately negative AoAs. The painted leading edge showed the same trends for AoAs greater than -3.5 degrees. Below an AoA of -3.5 degrees the baseline IR images with the painted leading edge, presented in Section 5, showed early transition with characteristic saw-tooth transition patterns, indicating a crossflow-dominated transition. The receptivity measurements confirmed this by measuring strong crossflow growth even with the DREs at very low heights. Under these conditions, DREs with a spacing less than $\lambda_{\text {crit }}$ can be applied to the painted leading edge to prevent the growth of the crossflow instability, thereby delaying transition.

Reibert et al. (1996) demonstrated that DREs with a spacing equal to $\lambda_{\text {crit }}$, or a multiple of $\lambda_{\text {crit }}$, could be used to promote early transition. In these experiments, multiple-mode crossflow waves were produced by increasing the spacing between the roughness elements. In those experiments, the most unstable crossflow wavelength was calculated to be $12 \mathrm{~mm}$. When roughness spaced $36 \mathrm{~mm}$ apart was applied to the leading edge, amplified modes with wavelengths of $36 \mathrm{~mm}, 18 \mathrm{~mm}, 12 \mathrm{~mm}, 9 \mathrm{~mm}, 7.2 \mathrm{~mm}, 6$ $\mathrm{mm}, 5.1 \mathrm{~mm}, 4.5 \mathrm{~mm}$, and $4 \mathrm{~mm}$ all appeared in the PSD calculations from data collected from 2-D hotwire scans. Hence superharmonics of the primary wavenumber were present, but subharmonics (higher wavenumbers) were not. Based on this remarkable discovery, DREs whose primary disturbance wavenumber does not contain a 
harmonic of the most unstable wavelength, can be applied to the swept-wing to delay transition. For this case, transition was delayed because the subcritical roughness prevented the growth of $\lambda_{\text {crit. }}$ (Saric et al. 1998). This same technique was applied to the SWIFT painted leading edge to delay transition back to the polished leading edge conditions. This Section focuses on the -4.69 degree AoA condition for SWLFC, for it has the most potential for dramatic increases in laminar flow.

\subsection{Linear Stability Theory Calculations}

Linear stability calculations were performed after completing the $C p$ measurements. Section 6 focused on wavelengths exhibiting the highest growth rate. The $4.5 \mathrm{~mm}$ wavelength proved to be the most unstable. When examining the $N$-factor plots in Figures 42 and 43, the shorter wavelengths exhibit stable behavior. In Figure 42 the wavelengths of $3 \mathrm{~mm}, 2.5 \mathrm{~mm}, 2.25 \mathrm{~mm}$, and $2 \mathrm{~mm}$ all grow initially, saturate, but then decay. Furthermore it is observed that these smaller wavelengths grow sooner; hence, the neutral point for these wavelengths are closer to the attachment line. Following the procedures employed in Saric et al. (1998), the same technique is applied to the SWIFT model to perform SWLFC. Considering both Figures 42 and 43, the $2.25 \mathrm{~mm}$ wavelength appears to be the best candidate for a SWLFC scheme. The $2.25 \mathrm{~mm}$ wavelength grows before the $4.5 \mathrm{~mm}$ wave. It appears to grow to a sufficient amplitude, enough to modify the mean flow, and then decays to nothing. Furthermore, the growth of the $2.25 \mathrm{~mm}$ wave is enough to modify the mean flow, but should not be enough to lead to transition. Its highest $N$-factor is just below 6. Baseline IR images with the polished 
leading edge showed that transition occurred near $N$-factors of 10 at the -4.69 degrees AoA condition. Figures 77 and 78 repeat the $N$-factor plots in Figures 42 and 43, with the exception that only the $4.5 \mathrm{~mm}$ and $2.25 \mathrm{~mm}$ wavelengths are isolated to focus on the behavior of these two wavelengths.

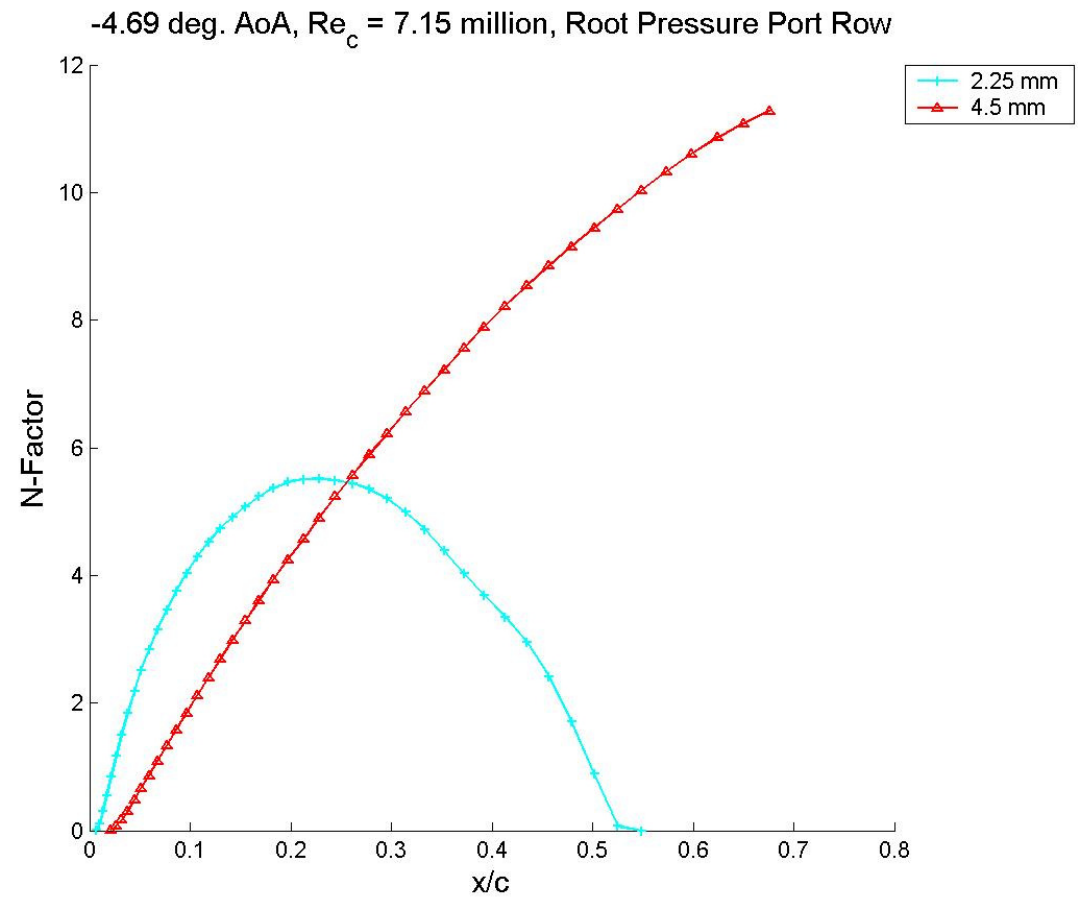

Figure 77: $N$-factor plot isolating the $4.5 \mathrm{~mm}$ and $2.25 \mathrm{~mm}$ wavelengths for the root pressure port row. 


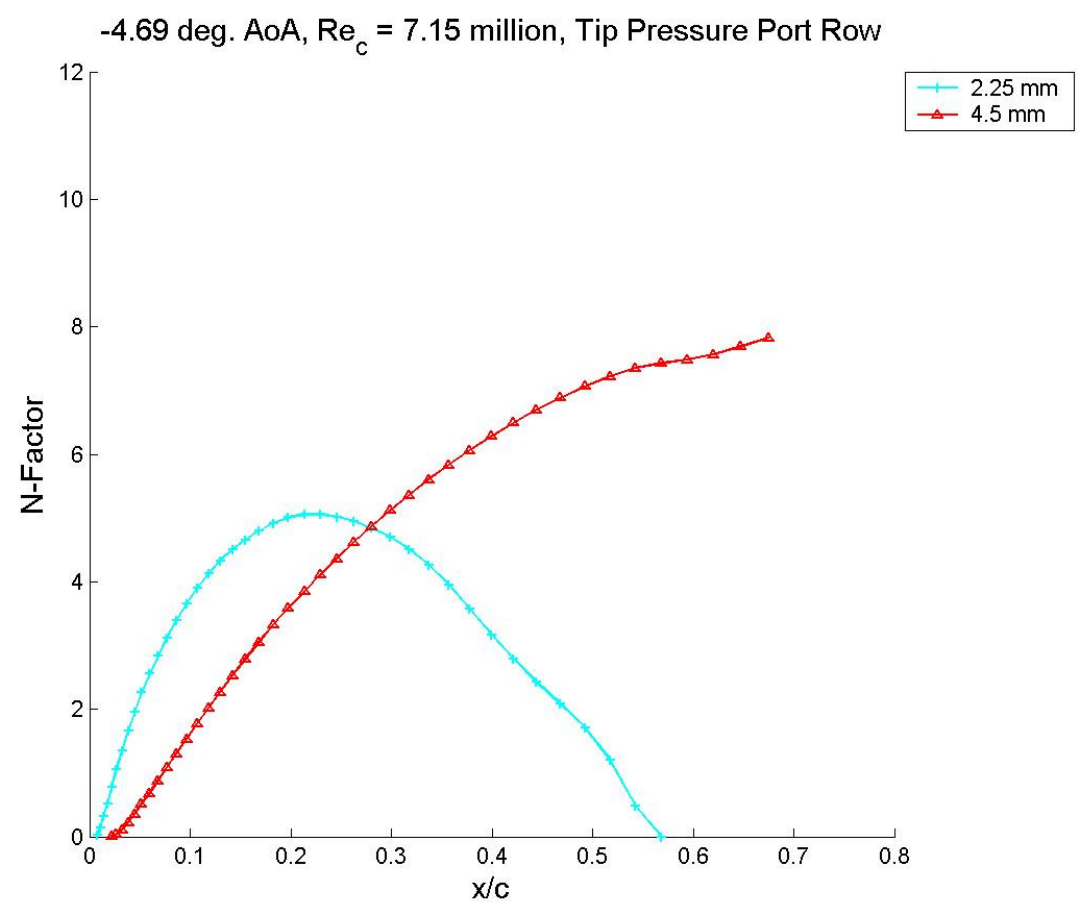

Figure 78: $N$-factor plot isolating the $4.5 \mathrm{~mm}$ and $2.25 \mathrm{~mm}$ wavelengths for the tip pressure port row.

Figures 77 and 78 show the $2.25 \mathrm{~mm}$ wavelength as a possible control wavelength. If roughness is applied at the $2.25 \mathrm{~mm}$ wavelength, measured parallel to the leading edge, then transition might be delayed. Unlike the experiments in Saric et al. (1998), where boundary-layer profiles were available with a 3-D hotwire traverse, these experiments only succeeded based on modification of the transition location. The technique is similar to the flight experiments of Saric \& Reed (2002). Furthermore, roughness height required to generate the growth exhibited in Figures 77 and 78 is unknown. As a result, roughness height has to be systematically increased until results, either positive or negative, are obtained. 


\subsection{Appliqué DREs Forward of Critical Neutral Point}

Prior to discovering the discrepancy with the air data boom on the starboard wing, the neutral point for the $4.5 \mathrm{~mm}$ wavelength was incorrectly calculated. This resulted in a neutral point for the $4.5 \mathrm{~mm}$ wavelength to be calculated as $1 \% \mathrm{x} / \mathrm{c}$ at the root and $1.3 \% \mathrm{x} / \mathrm{c}$ at the tip pressure port row. With these neutral point calculations, control appliqué DREs were placed at these locations. The diameter of the control DREs were $1 \mathrm{~mm}$ with a spacing of $2.25 \mathrm{~mm}$, resulting in a $d / \lambda$ ratio of 0.44 .

As discussed in Section 5, the polished leading edge demonstrated large amounts of NLF and did not need the control DREs. As a result control DREs were only demonstrated on a painted leading edge with early transition. However, the painted leading edge presented in Section 5 is not equivalent to the one presented here. The surface roughness of the painted leading edge presented in this section was measured to be $1.15 \mu \mathrm{m}$ rms with $3.63 \mu \mathrm{m}$ average peak-to-peak values.

Figure 79 shows IR images of two different flights. The image on the left shows early transition at $30 \% x / c$ with the painted leading edge. Subcritical spacing at $2.25 \mathrm{~mm}$ was applied on the leading edge at a root neutral point of $1 \% x / c$ and a tip neutral point of $1.3 \% x / c$. First roughness heights of $6 \mu \mathrm{m}$ were attempted. However, no change in the baseline transition was observed. Another layer of appliqué DREs was applied, but this time transition moved back to $60 \% x / c$, demonstrating a $100 \%$ increase in laminar flow. 
These results were very promising. For the next dive the $A o A$ was decreased to -2.5 degrees. However, at this condition improvement in the baseline IR image was not observed and the IR image looked much like the baseline IR image at that AoA. Furthermore, the roughness height was increased to $k=18 \mu \mathrm{m}$, but the transition location looked worse than the baseline.

$\underline{\mathrm{k}=0 \mu \mathrm{m}}$

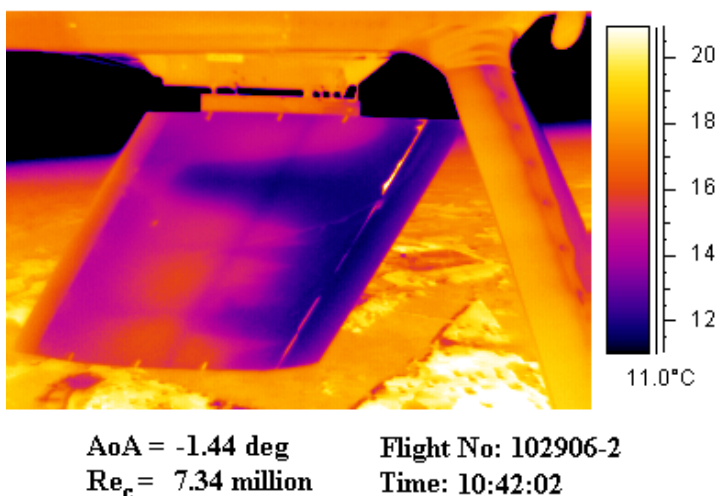

$\underline{k}=12 \mu \mathrm{m}$

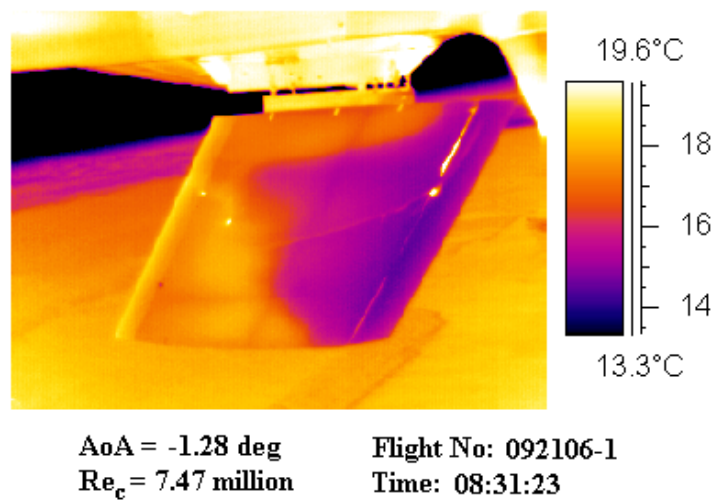

Figure 79: Demonstration of SWLFC with [12/2.25|1] (Root $R e_{k}=1.9$, Tip $R e_{k}=2.1$, $\left.k / \delta_{\text {root }}=0.05, k / \delta_{\text {itp }}=0.05\right)$ control DREs.

The baseline IR image on the left in Figure 79 shows transition at $30 \% x / c$ at a stable $A o A$ of only -1.44 degrees. Based on the transition Reynolds number plot given in Figure 39, it appears that this leading edge must have extremely high roughness compared to the different paint technique presented in Section 5. However, the surface roughness of the leading edge presented in Figure 79 is not substantially higher than that described in Section 5; in fact the surface roughness is slightly lower. This discrepancy in the data can be explained by examining AoA errors. The flights performed in 2006 
were all conducted with the air data boom measuring $A o A$ on the starboard wing. It was shown in Rhodes et al. (2008) that the air data boom had large positioning error. Despite correcting the AoAs to the values presented in Figure 79, there is probably still a 2degree error in the data. The baseline image in Figure 79 looks more representative of a 3.5 degree $A o A$ condition, based on Figure 39. Despite being unclear on the actual $A o A$, the two IR images in Figure 79 can still be compared because both should have similar $A o A$ errors, which can be neglected when performing a side-by-side comparison. In light of the large uncertainty in the $A o A$, decisive conclusions can not be inferred from these results. The only conclusion worth mentioning is that the $2.25 \mathrm{~mm}$ spacing of the DREs and the $x / c$ location appear to be effective at delaying transition. However, the results should be repeated with correct measurements of $A o A$ with the port-mounted five-hole probe in order to report definitive results.

\subsection{Variable Height DREs}

Based on the promising SWLFC results displayed in Figure 79, the leading edge was modified to accept the DRE insert first described in Section 6. However, for the control case, holes were drilled into the insert at a spacing of $2.25 \mathrm{~mm}$ with a diameter of $1 \mathrm{~mm}$, resulting in twice as many variable-height DREs. The reasons for the leadingedge modification were twofold. First, it is difficult to compare transition data taken from one flight and compare it with transition data for another flight with DREs added. Boundary-layer transition is dependent on too many variables which cannot be perfectly matched for each flight. Therefore, it is advantageous to have an active control system 
where both the baseline and artificial roughness conditions can be tested during the same flight. Variable height DREs allow such measurements because pressure is turned off for the baseline image, and then the DREs are pressurized for a real-time comparison. Secondly, the receptivity measurements described in Section 7 were conducted with the variable-height roughness setup.

A second DRE insert was machined with the holes drilled at the $1.3 \% \mathrm{x} / \mathrm{c}$ location at the tip and $1 \% x / c$ location at the root. Like the results presented in Figure 79, the hole diameters were $1 \mathrm{~mm}$ and the roughness spacing was $2.25 \mathrm{~mm}$. Figure 80 shows one flight demonstrating success with this setup. The reader is reminded that the variable-height DREs have a different cross section compared to the appliqué DREs. Figure 57 shows a cross-sectional comparison between the two types of roughness. 


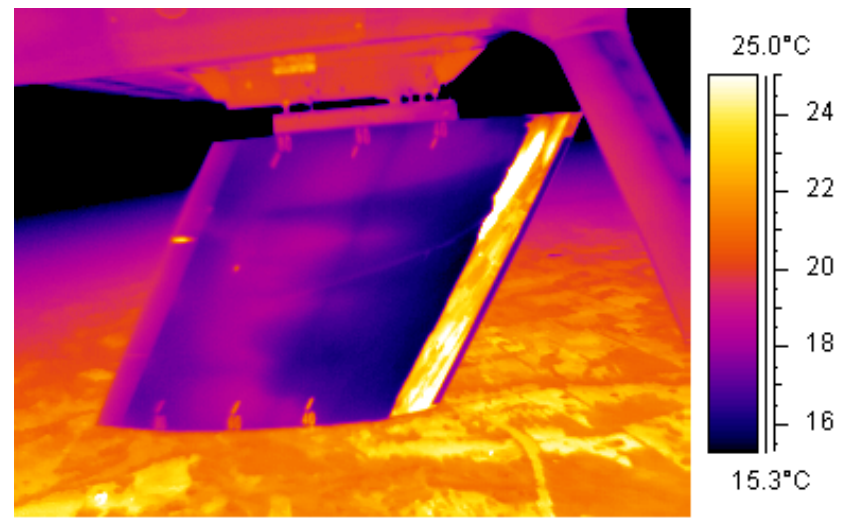

$$
\begin{array}{ll}
\mathrm{AoA}=-2.56 \mathrm{deg} & \mathrm{k}=25 \mu \mathrm{m} \\
\mathrm{Re}_{\mathrm{c}}=7.45 \text { million } & \text { Time: } 10: 42: 55
\end{array}
$$

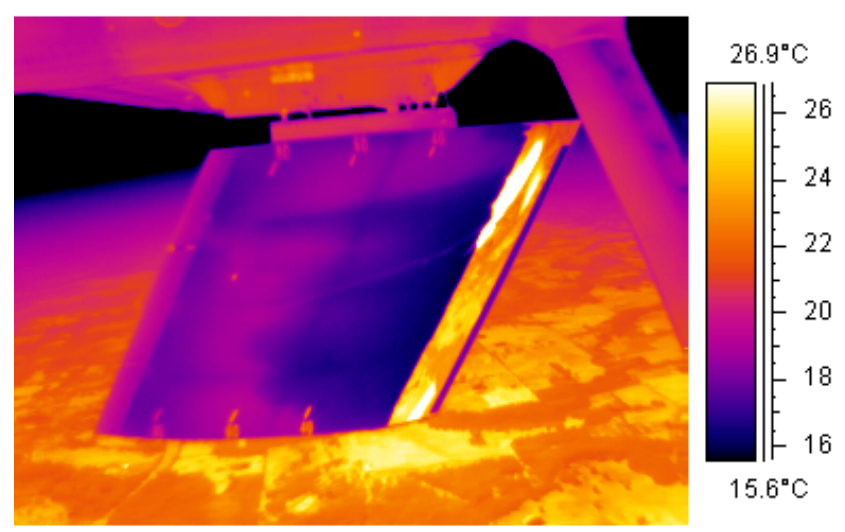

Freestream conditions unknown $\mathrm{k}=25 \mu \mathrm{m}$

Time: 10:43:11

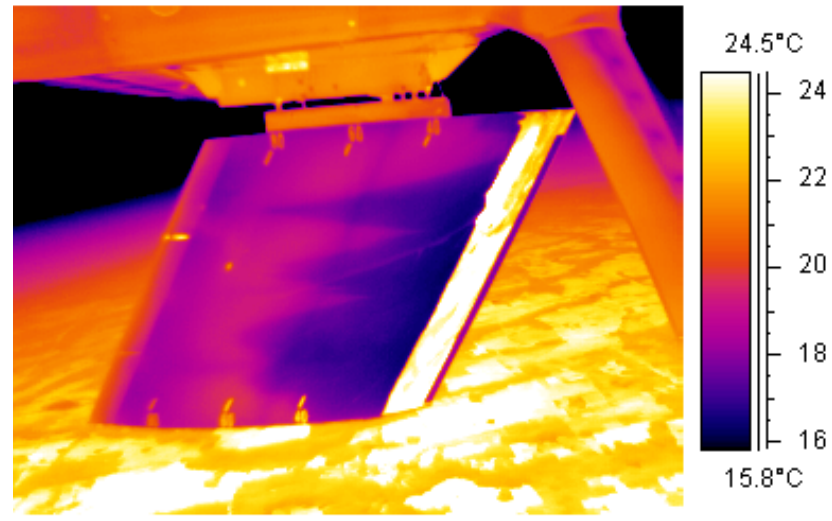

$$
\begin{array}{ll}
\mathrm{AoA}=-2.49 \mathrm{deg} & \mathrm{k}=0 \mu \mathrm{m} \\
\operatorname{Re}_{\mathrm{c}}=7.47 \text { million } & \text { Time: } 10: 43: 22
\end{array}
$$

Figure 80: Demonstration of SWLFC with [25|2.25I1] (Root $R e_{k}=9.0$, Tip $R e_{k}=10.3$, $\left.k / \delta_{\text {root }}=0.10, k / \delta_{\text {tip }}=0.10\right)$ control, variable-height DREs. 
In Figure 80, the two large wedges in the bottom IR image were generated by bolts on the back side of the model being torqued too high. These bolts were added to hold the DRE insert after modifying the leading edge for variable-height DREs. At the time, the source of the two wedges was unknown, but after post processing the data it was clear that these two wedges were created by bolts on the back side of the leading edge, closest to the attachment line. The spacing and location of these two wedges are directly in line with two of the bolts on the non test side of the model. However, the top two images show that the wedges disappeared when the variable-height DREs were pressurized up to $25 \mu \mathrm{m}$. Prior to reaching roughness heights of $25 \mu \mathrm{m}$, the DRE insert was slowly pressurized from $k=0 \mu \mathrm{m}$. Below $k=25 \mu \mathrm{m}$, the two wedges were present. Once the roughness heights were increased to $k=25 \mu \mathrm{m}$ the two wedges disappeared. The top two images in Figure 80 still show residual heat left from the turbulent wedges. The high thermal inertia of the SWIFT model means changes observable with the IR camera are slow. However, once the DREs were turned off, the two turbulent wedges reappeared and are much more visible in the third image of Figure 80, thereby providing more evidence that the boundary layer was laminar with the DREs pressurized. Note that the second IR image shows the most improvement, but the details of the freestream conditions are unknown. Only the time and the roughness height are known for that image.

The two wedges displayed in the bottom image in Figure 80 appear to be similar to the wedges generated by the isolated roughness elements experienced in the 
Radeztsky et al. (1993a) experiments. However, in this case, the two wedges were not created by cylindrical roughness elements but were created by bolts warping the aluminum leading edge. Upstream of the two wedges, $\lambda_{\text {crit }}$ is excited in the form of a wave packet. With the control DREs pressurized, the growth of the $2.25 \mathrm{~mm}$ wavelength prevents the growth of $\lambda_{\text {crit }}$, thereby delaying transition. To prevent similar wedges in the future, all leading edge attachment bolts and DRE insert bolts were never torqued above 25 in-lb. This procedure solved the problem.

Once the wedges were eliminated due to the lower torque values, SWLFC was performed on more uniform transition fronts, and not just turbulent wedges. However, no signs of a delayed transition were observed. Roughness heights were pushed well beyond the elastic limit of the tape, but no changes in transition were observed, not even a boundary-layer trip was recorded. Vacuum was also attempted, but still no results. Furthermore, the results presented in Figure 80 were conducted with the polished leading edge with the DRE insert. However, this leading edge was also painted in order to control more uniform transition fronts, but no results were achieved with this case either.

The IR images in Figure 80 were recorded with the air data boom on the starboard side. After realizing the error in the AoA measurements, and correcting them with the five-hole probe, the stability calculations were repeated with new experimental $C p$ measurements. The calculations presented in Figures 40 through 43, 77, and 78 were made using the $C p$ measurements with correct $A o A$ values. Based on these figures, the neutral point for the $4.5 \mathrm{~mm}$ wavelength is calculated to be $2.07 \%$ at the root and $2.41 \%$ 
$x / c$ at the tip. The receptivity measurements presented in Section 7 confirmed that critically-spaced roughness placed at this location excited the crossflow instability. As a result, this newly calculated neutral point was tested to demonstrate more convincing results than those presented in Figures 79 and 80.

\subsection{Appliqué DREs at the Critical Neutral Point}

Control DREs, equivalent to those tested in Figure 79, were applied to the painted leading edge at the $2.07 \% x / c$ root and $2.41 \% x / c$ tip location. For this case [12/2.25|1] appliqué DREs were applied on top of the unpainted DRE insert tape with zero differential pressure applied. Figure 81 shows two IR images taken during the same dive. Below $R e_{c}=7.2$ million, three distinct wedges are observed, in addition to a bug strike wedge which is constant for both images. Above $R e_{c}=7.2$ million, the three wedges disappear, which was unlike anything seen before. If a few of the DREs were tripping the boundary layer, an increase in the $R e_{c}$ would make it worse, not better.

It is counter intuitive that an increase in $R e_{c}$ would increase the amount of laminar flow. The IR images taken in Figure 81 were taken at a stable $A o A$ near -2.61 degress. Without these three wedges and the bug strike, the transition location would be near $80 \% x / c$. The $C p$ measurements, and therefore the stability calculations, for this test

point were made at $R e_{c}=7.08$ million. It is possible that below this $R e_{c}$ the appliqué DREs had a destabilizing effect. Above the calculated $R e_{c}$ the $2.25 \mathrm{~mm}$ wavelength is 
calculated to be stabilizing, resulting in more laminar flow. The results of this test point are still not entirely understood.

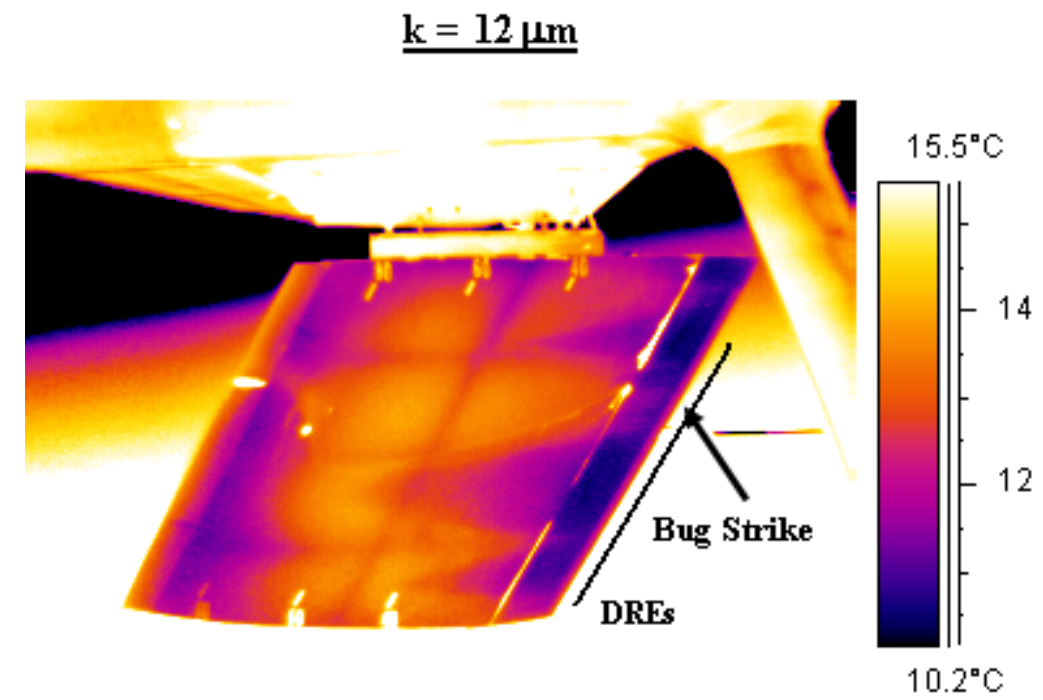

$$
\begin{array}{ll}
\text { AoA }=-2.40 \text { deg } & \text { Flight No: } 083008-1 \\
\operatorname{Re}_{c}=7.10 \text { million } & \text { Time: } 10: 35: 35
\end{array}
$$

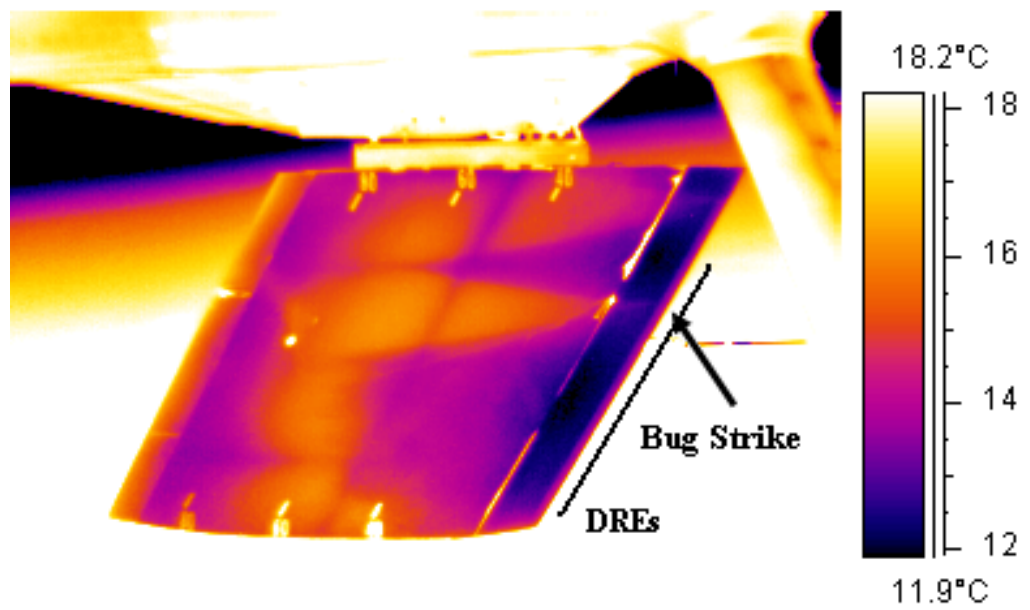

$$
\begin{array}{ll}
\text { AoA }=-2.52 \text { deg } & \text { Flight } N_{0}: 083008-1 \\
\operatorname{Re}_{c}=7.29 \text { million } & \text { Time: } 10: 36: 11
\end{array}
$$

Figure 81: Demonstration of SWLFC with [12|2.25|1] (Root $\operatorname{Re}_{k}=1.9, \operatorname{Tip} \operatorname{Re}_{\mathrm{k}}=2.1$, $\left.\mathrm{k} / \delta_{\text {root }}=0.05, \mathrm{k} / \delta_{\text {tip }}=0.05\right)$ control, appliqué DREs. 


\subsection{Appliqué DREs at the Control Neutral Point}

Control roughness applied at the critical neutral point did not produce any results beyond those presented in Figure 81. Roughness heights up to $k=30 \mu \mathrm{m}$ were tested, but no laminarization was observed. It was assumed that the $2.25 \mathrm{~mm}$ wavelength was not growing early enough to prevent the growth of the $4.5 \mathrm{~mm}$ wavelength. Figures 77 and 78 show that the $2.25 \mathrm{~mm}$ wavelength grows prior to the $4.5 \mathrm{~mm}$ wavelength, however, it might be beneficial to place the control DREs at the control neutral point to guarantee that that wavelength grows prior to the $4.5 \mathrm{~mm}$ wavelength. The neutral point for the $2.25 \mathrm{~mm}$ wavelength is calculated to be $0.6 \% x / \mathrm{c}$ for the -4.69 degree $A o A$ condition. Applique DREs were placed at this location to further demonstrate SWLFC with control DREs. The painted leading edge for this case is equivalent to the one presented in Section 5 with the baseline IR, therefore the transition location over a wide range of AoAs is well known. Furthermore, the $A o A$ measurements were correctly recorded with the five-hole probe so no corrections were required.

During these experiments, the flight profile included a dive testing all AoAs of interest. First the dive was initiated at -2.61 degrees $A o A$ until the targeted $R e_{c}$ of 7.5 million was recorded and displayed for the pilot. Then the pilot would roll in the rudder trim wheel slowly until the $A o A$ was -4.69 degrees. In this manner, all AoAs could be tested during a single dive. 
First [6/2.25|1] appliqué DREs were applied at the $0.6 \% x / c$ location but no change from the baseline image was observed. The roughness height was then increased to $k=12 \mu \mathrm{m}$. For this case transition was delayed from AoAs of -3.53 degrees out to 4.56 degrees AoA. At -5.21 degrees AoA transition moved forward very quickly, catching up to the baseline painted transition location. Figure 82 shows a comparison between the AoAs tested in Section 5 with no roughness and the flight with [12|2.25|1] roughness, showing an increase in the amount of laminar flow for three of the four test conditions.

There is a substantial increase in the amount of laminar flow above mid-span. As much as $100 \%$ more laminar flow is recorded at mid-span. The region near the tip pressure port row shows early transition, and not any real improvement over the baseline image. The roughness height was then increased to $k=18 \mu \mathrm{m}$ in an attempt to stabilize the tip pressure port region. However, for this case there was no improvement in that region, and even the mid-span region got worse near the -4.69 degree $A o A$ condition. 
Flight No: 090408-1, k=0 $\mu \mathrm{m}$

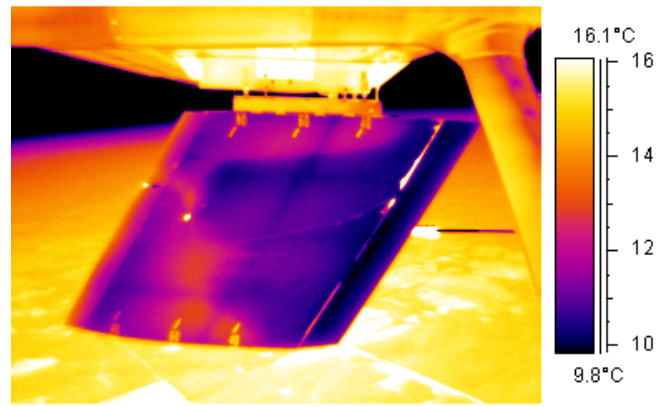

AoA $=-3.55 \mathrm{deg}, \operatorname{Re}_{\mathrm{c}}=7.22$ million, Time: 09:25:25

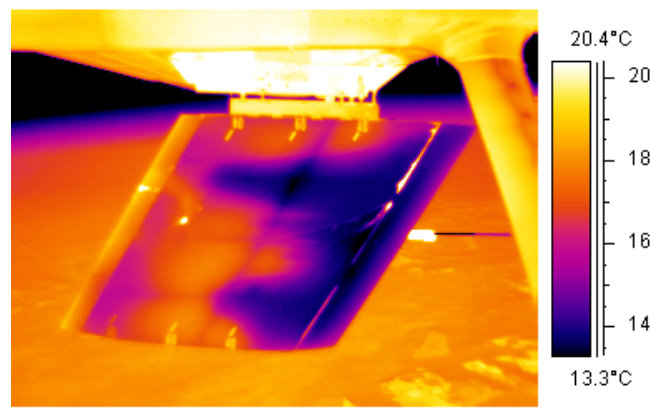

AoA $=-3.96 \mathrm{deg}, \mathrm{Re}_{\mathrm{c}}=7.47$ million, Time: 09:27:15

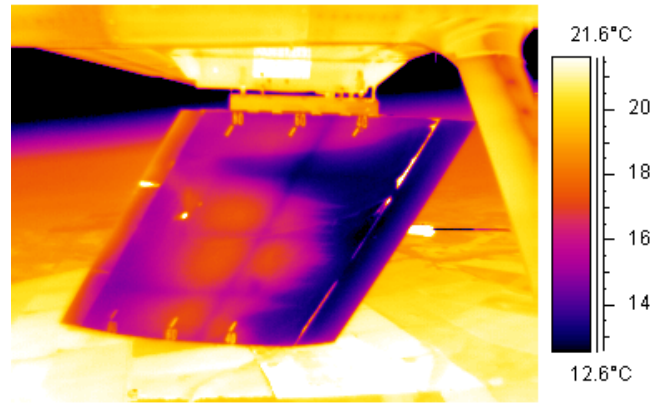

AoA $=-4.61$ deg, $\operatorname{Re}_{c}=7.47$ million, Time: 10:08:26

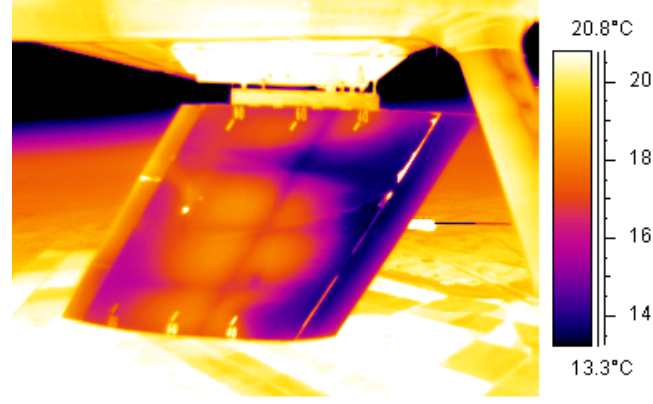

AoA $=-5.07$ deg, $R_{c}=7.38$ million, Time: 10:08:30
Flight No: 101808-1, k=12 $\mu \mathrm{m}$

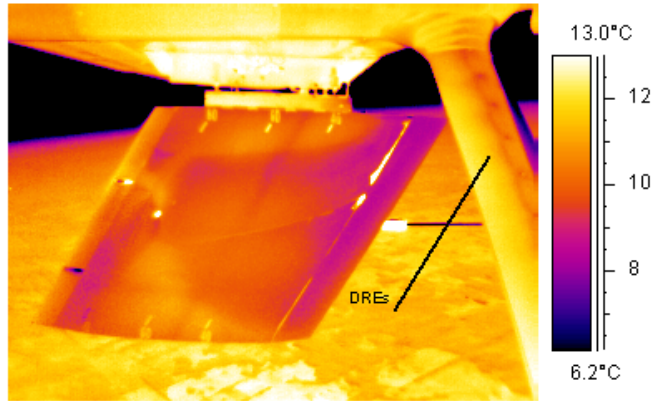

AoA $=-3.53 \mathrm{deg}, \operatorname{Re}_{\mathrm{c}}=7.32$ million, Time: 09:29:10

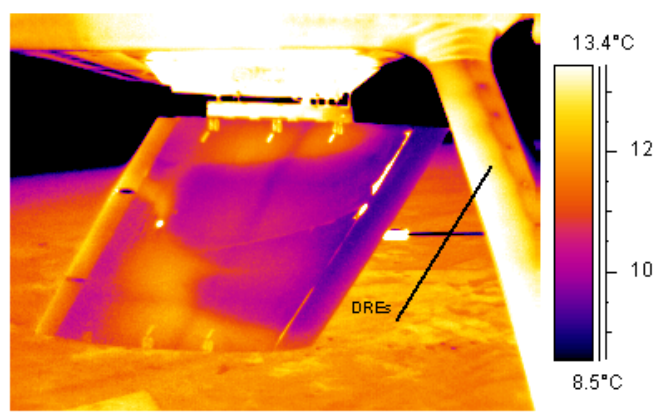

AoA $=-4.12 \mathrm{deg}, \mathrm{Re}_{\mathrm{c}}=7.44$ million, Time: 09:29:41

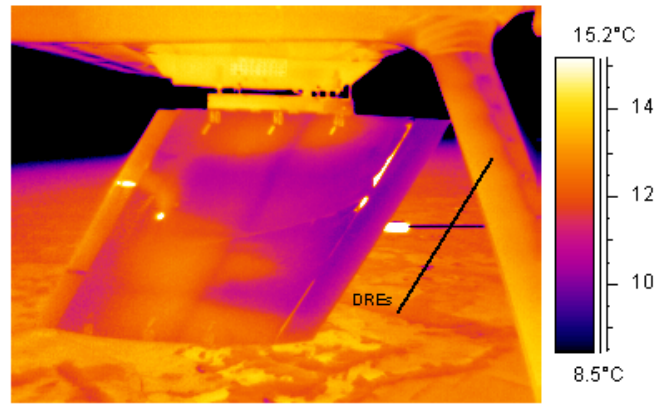

AoA $=-4.56$ deg, $\operatorname{Re}_{c}=7.51$ million, Time: 09:30:24

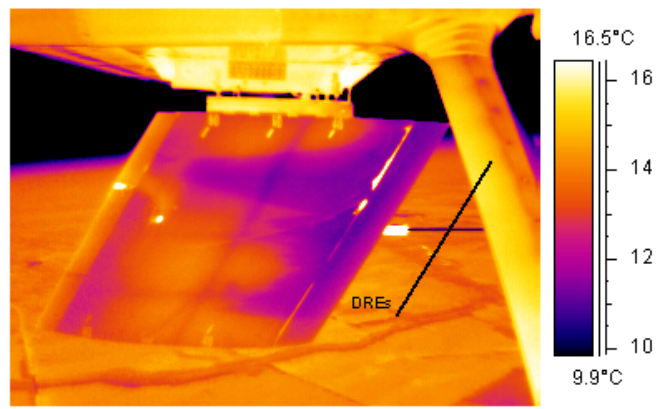

AoA $=-5.21$ deg, $\operatorname{Re}_{c}=7.49$ million, Time: 09:31:02

Figure 82: Demonstration of SWLFC with [12/2.25l1] (Root $R e_{k}=1.9$, Tip $R e_{k}=2.1$, $\left.k / \delta_{\text {root }}=0.05, k / \delta_{\text {tip }}=0.05\right)$ control, appliqué DREs at the control neutral point. 
After demonstrating promising results in Figure 82, the leading edge was removed to repeat the surface roughness measurements. Particularly, more attention was paid towards measuring the surface roughness at the tip pressure port row and comparing it with the surface roughness at the root pressure port row. The average surface roughness of this painted leading edge measured along the entire span at $1 \% x / c$ was $1.66 \mu \mathrm{m}$ rms with $7.69 \mu \mathrm{m}$ average peak-to-peak values. Surface roughness measurements were then repeated forward and aft of the DREs located at $0.6 \% x / c$. The root surface roughness measured forward and aft of the DREs and then averaged was recorded to be $0.45 \mu \mathrm{m}$ rms with $0.94 \mu \mathrm{m}$ average peak-to-peak values. This procedure was then repeated at the tip pressure port row with average values recorded to be 1.12 $\mu \mathrm{m}$ rms with $2.12 \mu \mathrm{m}$ average peak-to-peak values. The roughness at the tip was approximately twice as high as the roughness at the root, possibly explaining why the DREs at the tip appeared to be ineffective at delaying transition.

Figure 83 shows surface scans over the DREs with the surface roughness tester. The top figure shows DREs applied to the polished aluminum leading edge. The data in the middle are for the root pressure port row with the painted leading edge, and the bottom image is for the tip pressure port row also for the painted leading edge depicted in Figure 82. There is a distinct difference between DREs applied to a polished surface versus a rough painted surface. There is an even larger difference between the scans performed near the root versus the tip corresponding to the IR image in Figure 82. Note that the region tested with the painted leading edge near the root pressure port actually 
had lower average peak-to-peak roughness values than the polished leading edge. However, the rms roughness was still lower for the polished leading edge. Figure 84 shows the IR image in Figure 82 at -4.12 degrees $A o A$ identifying where the two roughness scans were performed depicted in Figure 83.
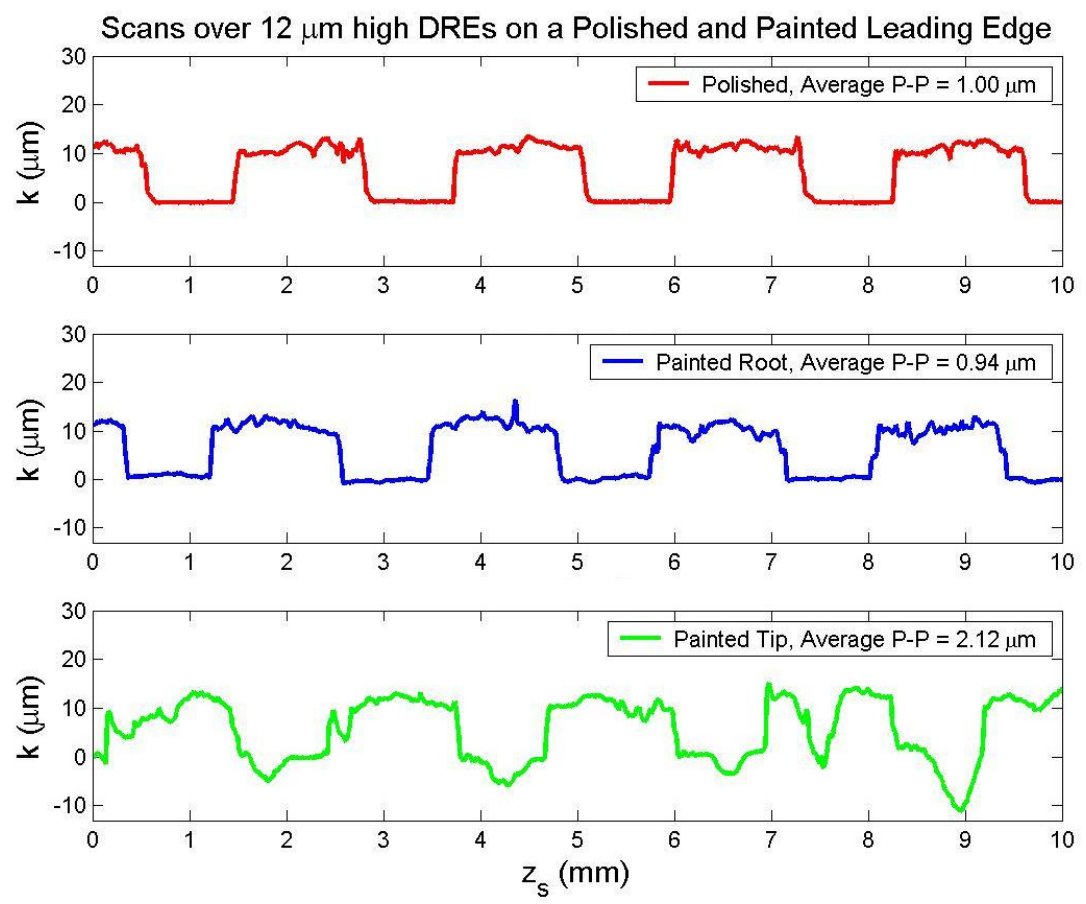

Figure 83: Surface scans over the appliqué DREs applied to the polished leading edge, painted leading edge near the root, and the painted leading edge near the tip. 


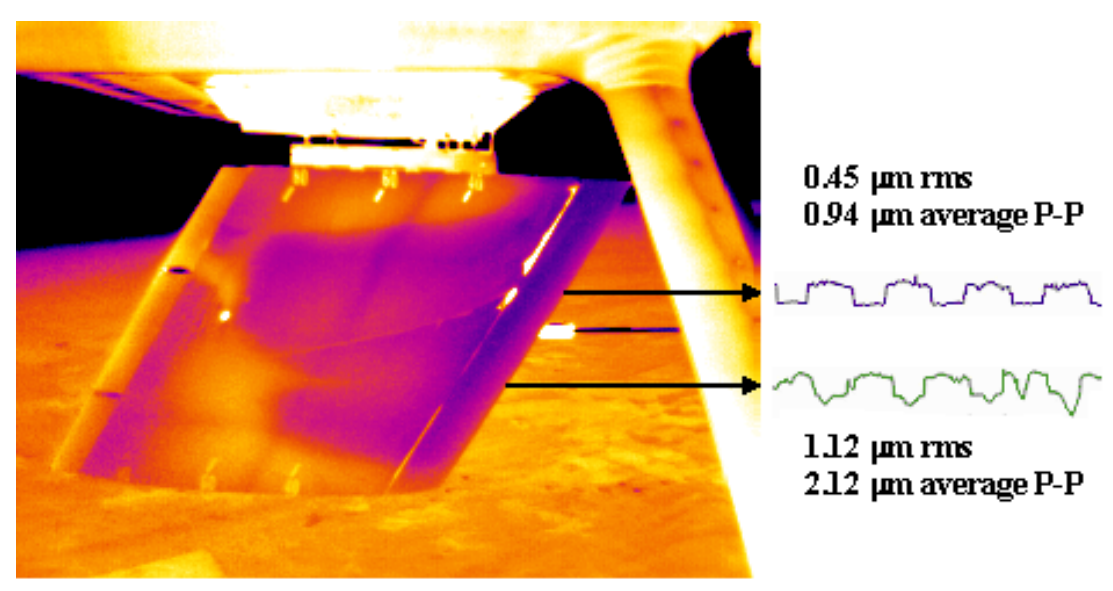

AoA $=-4.12 \mathrm{deg}, \operatorname{Re}_{c}=7.44$ million, Time: 09:29:41

Figure 84: Schematic showing where the two different roughness scans were performed for Figure 83.

Visually there is a significant difference between the tip and root DRE scans depicted in Figure 83. Particularly at $z_{s}=7.5 \mathrm{~mm}$ and $z_{s}=9 \mathrm{~mm}$ there are large valleys in the paint. The ink-like consistency of the appliqué DREs is soft enough to fill in large valleys and not span across them, as a rigid roughness element would do. As a result, the substrate that the appliqué DREs are applied to must be very uniform if any significant SWLFC is desired.

Based on the surface scans measured in Figure 83, there could possibly be even more promising results with a polished leading edge at an unstable negative $A o A$ condition. During the receptivity measurements described in Section 7, appliqué control DREs were applied at the bottom section of the model, which was not being used for those measurements. [12/2.25I1] appliqué DREs were placed over the tape of the DRE 
insert at the $0.6 \% x / c$ location. However, transition was not delayed at this roughness height, contrary to the results obtained in Figure 82.

\subsection{Swept Wing Laminar Flow Control Summary}

A comparison of the transition Reynolds number can be made for the polished leading edge, painted leading edge, and now the two control cases demonstrating promising results. Figure 85 shows this plot over a wide range of AoAs. The [12/2.25|1] control DREs placed at the control neutral point of $0.6 \% x / c$ were effective at delaying transition from -3.5 degrees $A o A$ until -4.56 degrees $A o A$. At -5.21 degrees $A o A$, the control DREs did nothing to increase the amount of laminar flow and transition moved quickly forward to the painted baseline condition. Thus the control DREs appear to be effective to a certain point, but then the neutral point changes enough to where the DREs cannot prevent the early transition. The control neutral point of $0.6 \% x / c$ was calculated for the -4.69 degrees $A o A$ condition, therefore the substantial change in transition location above this $A o A$ confirms the stability calculations. 


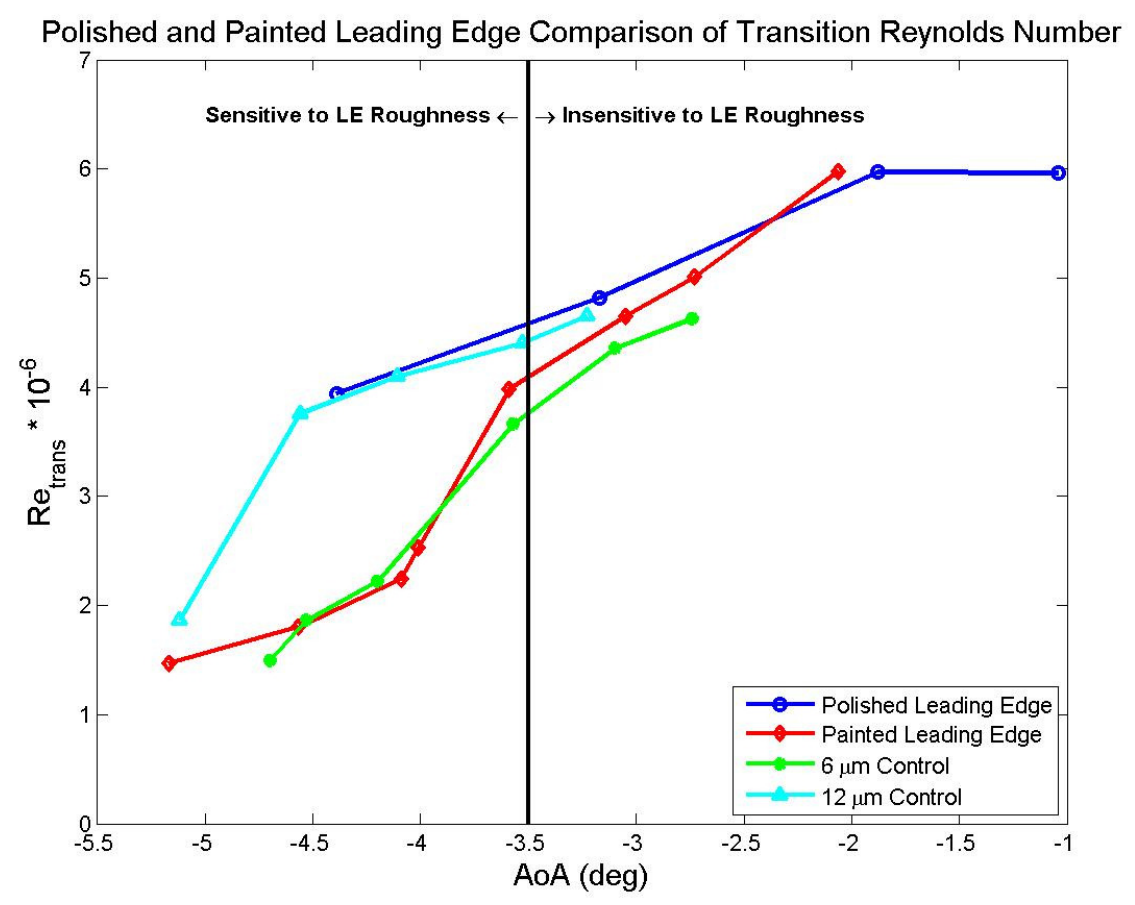

Figure 85: Plot of transition Reynolds number for the polished leading edge and painted leading edge with and without [k|2.25|1] control, appliqué DREs.

After reviewing all flights demonstrating successful SWLFC, the appliqué DREs were only effective at $k=12 \mu \mathrm{m}$ with a painted leading edge. The variable-height DREs were only effective at $k=25 \mu \mathrm{m}$ with a polished leading edge. Based on these statistics, the appliqué DREs appear to be effective at delaying transition with half the roughness height. Furthermore, successful flights with control DREs were few and far between. 112 flights were dedicated towards demonstrating SWLFC with control DREs. Out of these 112 flights only six of them successfully demonstrated SWLFC. However, the conditions displayed in Figure 82 were repeated on a second flight with [12|2.25|1] appliqué DREs. This leading edge also demonstrated some success with the [18|2.25|1] 
appliqué DREs. Therefore the leading edge in Figure 82 shows promise for future tests, especially if the tip pressure port region at $0.6 \% x / c$ is sanded down with 1000 -grit sandpaper to match the surface roughness at the root pressure port row. 


\section{OPERATIONAL RECOMMENDATIONS}

Inevitably operational vehicles employing LFC will need a system that is optimized for various AoAs. During the cruise portion of flight the gross weight of the vehicle decreases as fuel is burned, resulting in a lower $A o A$ on the wing. As a result, for the case of DREs, the system would require a dynamic system to move or modulate both location and height of the DREs as flight conditions change. Figure 85 showed that the location and height of the control DREs were only effective up to a point. Beyond -4.5 degrees $A o A$ the DREs were no longer effective. There are operational difficulties in the pneumatic system. It is feasible that there can be several rows of pneumatic DREs and a set of diverting valves can be used to move one pressure chamber to another. However, some work is required to make the flexible membrane robust enough to be used in an operational environment.

Currently the effectiveness of the control DREs over a large range of AoAs is unknown. If future experiments show that the control DREs are optimized for one $A o A$, but are also effective over a large range of AoAs, then fixed roughness could be applied to an operational vehicle. Roughness of a fixed shape, height, and location would be more robust for an operational aircraft. Furthermore, mission profiles can be tailored for maintaining a constant $A o A$ on the vehicle resulting in a slight climb throughout the mission as fuel is burned. This type of profile lends itself for more promising results with DREs. 


\subsection{Insect Contamination}

No attempt was made to eliminate bug strikes during these experiments. However, the number and location of bug strikes was recorded for every flight to provide statistics for the flight-testing community. A 56-inch segment of the O-2A wing above the SWIFT model was cleaned prior to each flight and the number and location of bug strikes was documented. The number and location of bug strikes was also documented for the SWIFT model. The bug strike results over the course of three years and 240 flights are presented here. Figures 86 through 91 present the bug strikes for both the O-2A wing and the SWIFT model as functions of different atmospheric variables. The bug strike totals have been normalized by the length of the test section in order to provide a meaningful comparison. The normalizing length for the SWIFT model is the span at 42 inches, and not the longer length measured parallel to the leading edge. The data show that the O-2A wing typically has a slightly higher number of bug strikes per linear foot. This could possibly be attributed to attachment line flow on the SWIFT model. Insects approaching the leading edge might be forced down the attachment line of the SWIFT model, thereby reducing the likelihood of an impact. This conjecture is purely speculation, so further investigation is required. However, this set of data may be the only one comparing the number of bug strikes on a straight wing compared to a swept-wing. The data here suggest that there is a $33 \%$ reduction in the number of bug strikes on the swept-wing when compared to a straight wing. Furthermore, it is unlikely that the reduced surface roughness on the highly-polished SWIFT leading edge is the source of the reduction. The flight experiments of Peterson \& Fisher (1978) 
demonstrated that super-slick and hydrophobic materials did little to prevent bug remnants from sticking to the surface.

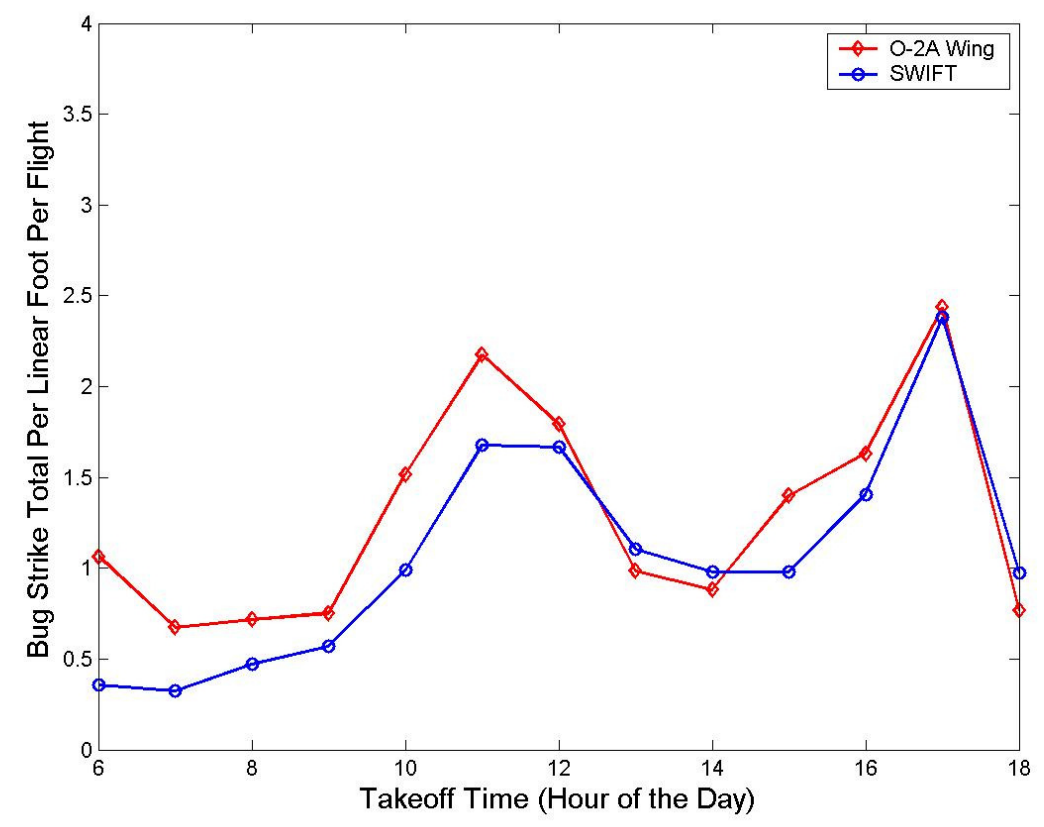

Figure 86: Bug strikes as a function of takeoff time. 


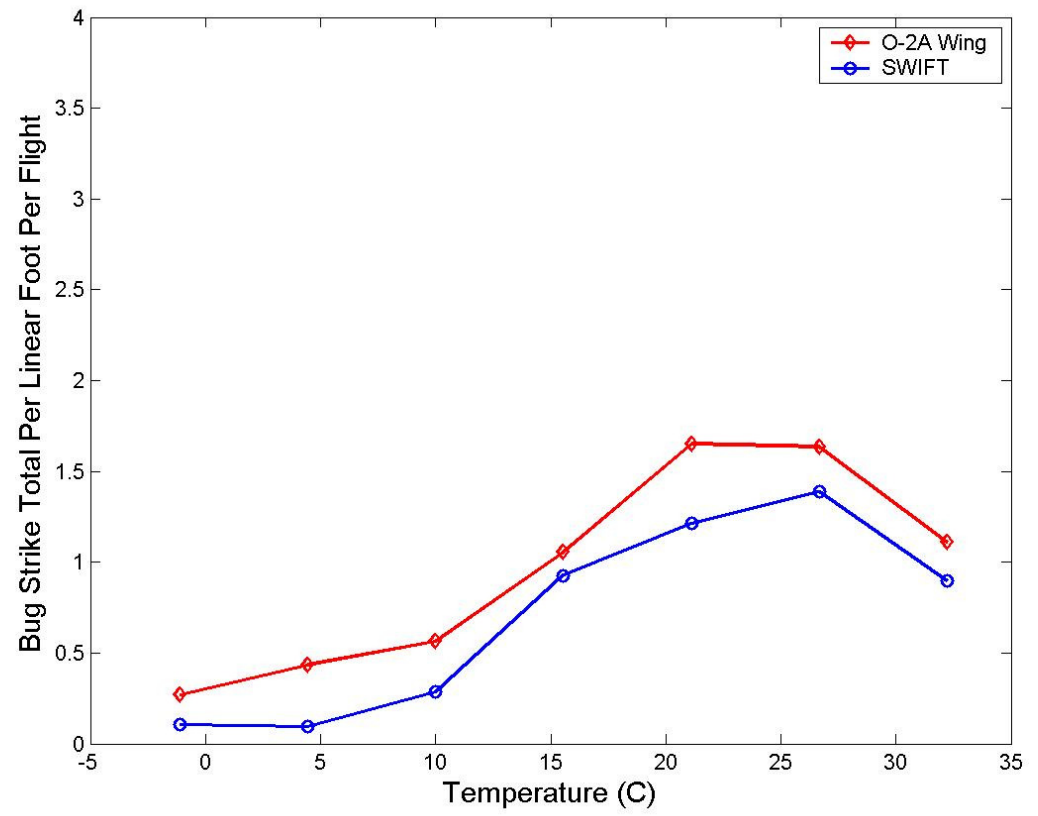

Figure 87: Bug strikes as a function of ground temperature.

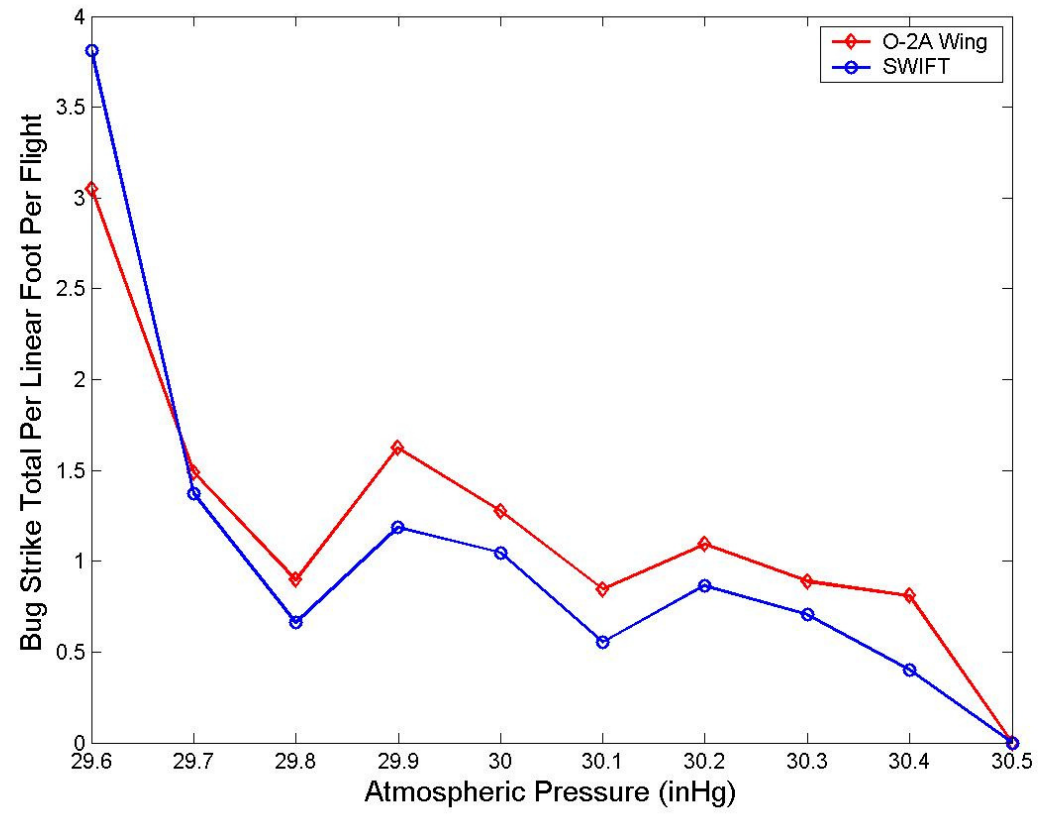

Figure 88: Bug strikes as a function of ground atmospheric pressure. 


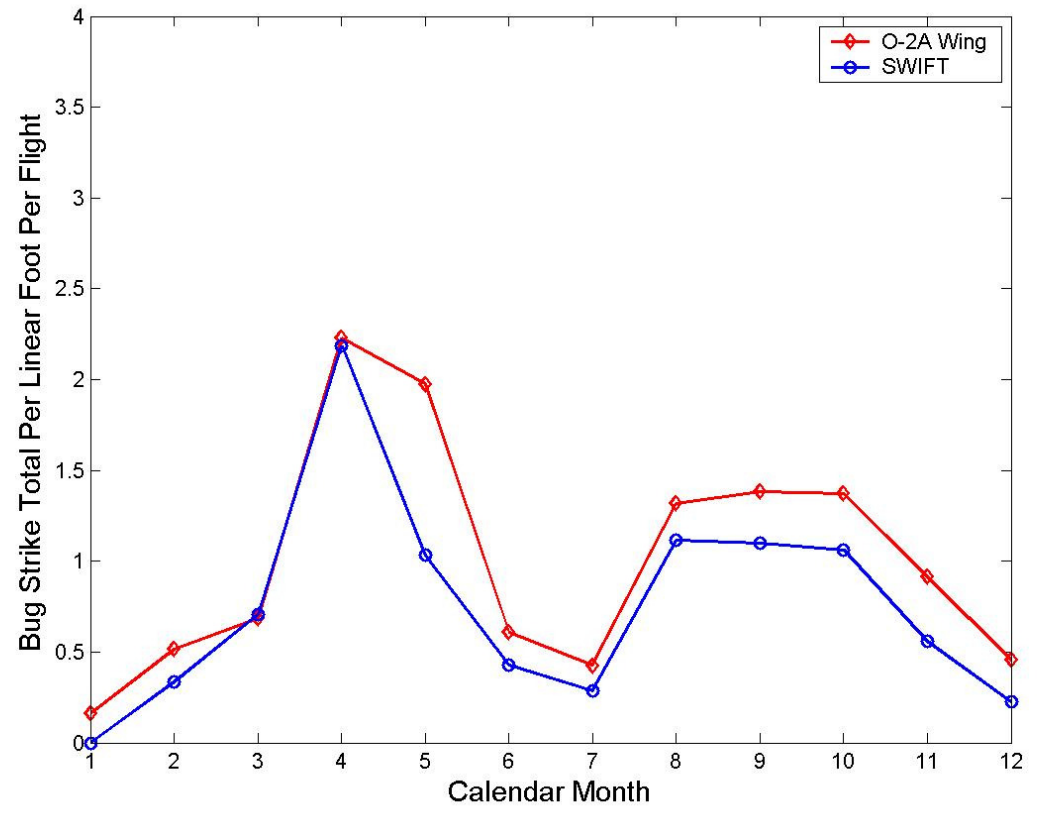

Figure 89: Bug strikes over the course of a calendar year.

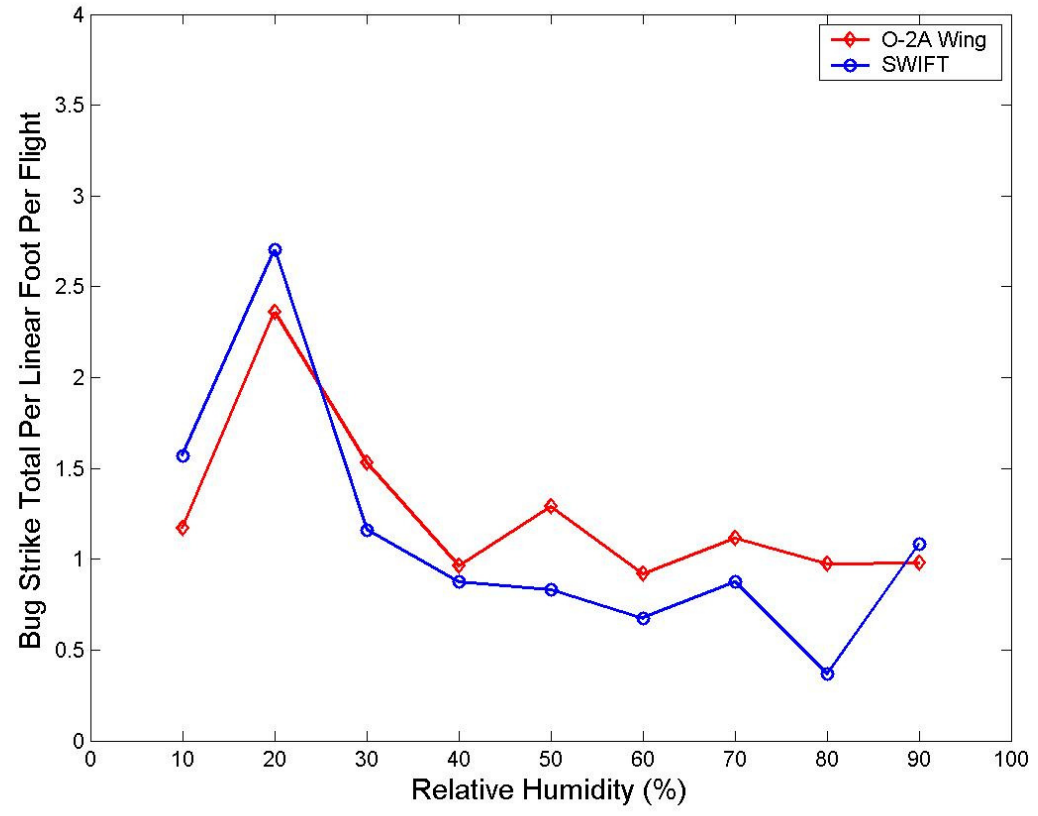

Figure 90: Bug strikes as a function of relative humidity. 


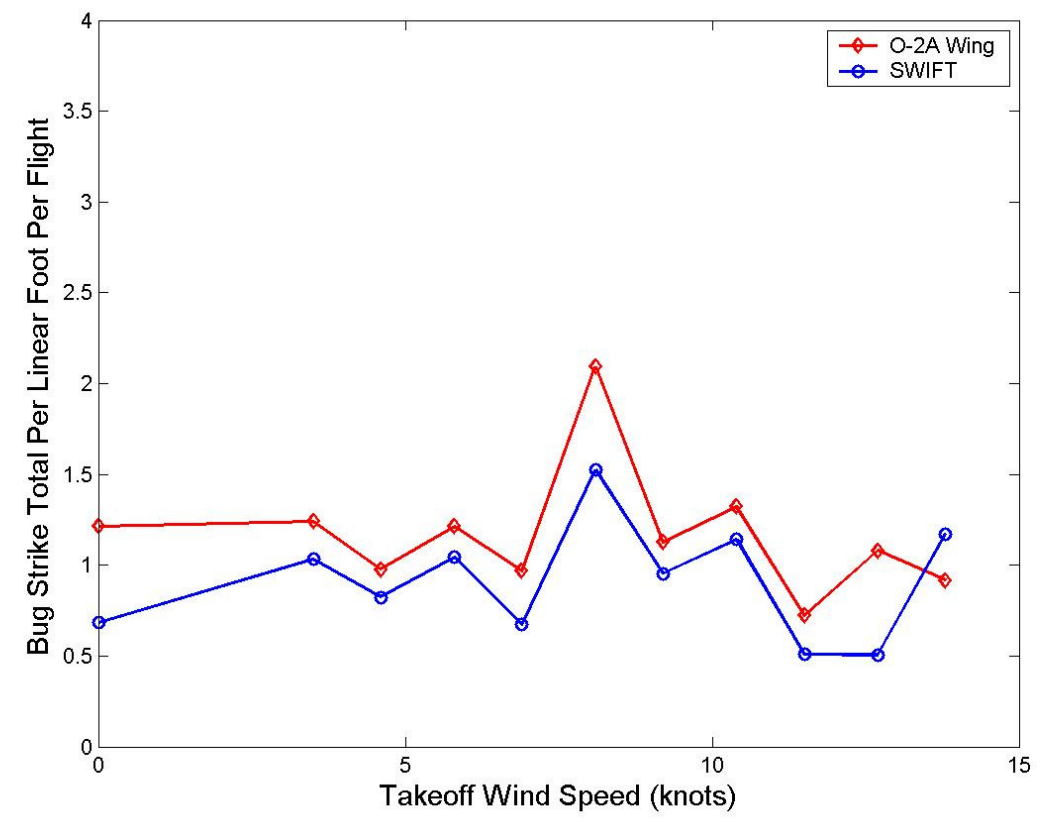

Figure 91: Bug strikes as a function of wind speed.

The data presented here agree well with the literature. Croom \& Holmes (1986) and Coleman (1961) both report the same trends observed here. The relative insect population density is a strong function of temperature, wind speed, and altitude. Past experiments showed that bug strikes were highest between the temperatures of $21^{\circ} \mathrm{C}$ and $27^{\circ} \mathrm{C}$ with a maximum at $25^{\circ} \mathrm{C}$. It was reported that below $21^{\circ} \mathrm{C}$ insect contamination was not a problem. The same trends were observed here, with the highest number of bug strikes collected around $24^{\circ} \mathrm{C}$ and $25^{\circ} \mathrm{C}$. The data collected based on the wind speed also compare with the literature. Here bug strikes are maximum when the wind speed was 8 knots. The literature quotes wind speeds from 3 to 8 knots as conditions for high insect population density. Lastly, there is a strong relationship between bug strikes and 
altitude. The majority of the bugs reside at altitudes below 250 feet, and above 500 feet, insect protection is not needed (Coleman 1961).

Data are also presented here on bug strikes as a function of takeoff time, month of the year, atmospheric pressure, and humidity. Takeoff time and month of the year show obvious trends, but both of these variables are temperature dependent. Bug strikes are minimized in the morning and in the early afternoon. These times correspond to the temperature lows and highs during the day, so temperature is really driving the data trends. Similarly bug strikes are minimized during the winter and summer months. This fact also is related to the temperature. However, Coleman (1961) reported no consistent correlations for insect population density with respect to barometric pressure or humidity. The data here appear to demonstrate a strong relationship between the number of bug strikes and atmospheric pressure. Bug strikes are minimized during high atmospheric pressure, as seen in Figure 88. Furthermore, bug strikes were maximized when the relative humidity was $20 \%$, but no strong trends appear to be present.

\subsection{Effect on Laminar Flow}

The heights of the bug strikes were never recorded, but a correlation between bugs with heights large enough to trip the boundary layer and those too small to generate a turbulent wedge was performed. Over the course of the 240 flights, the SWIFT model collected a total of 699 bug strikes on the leading edge. Out of the 699 bug strikes, it can be assumed that half of them were picked up during takeoff and the other half were 
collected on landing. Out of the 350 bug strikes assumed to be collected during takeoff, only 28 of these generated turbulent wedges on the SWIFT model. Thus there was an $8 \%$ chance of hitting a bug of large enough size to produce a turbulent wedge, based on a $R e_{c}$ of 7.5 million. Assuming a test condition where the model was laminar back to $80 \% x / c$, a bug strike large enough to produce a turbulent wedge reduces the percentage of laminar flow by $17.8 \%$. This calculation is based on the suggestions of Saric (2007), that a turbulent wedge propagates downstream taking the form of a 12-degree half-angle wedge. Figure 92 shows the percentage loss of laminar flow due to a bug strike. Based on these assumptions, the data can be re-plotted in Figures 93 through 96, now with the percentage loss of laminar flow displayed, rather than bug-strike totals. Plots with strong relationships to temperature have been eliminated to reduce redundancy.

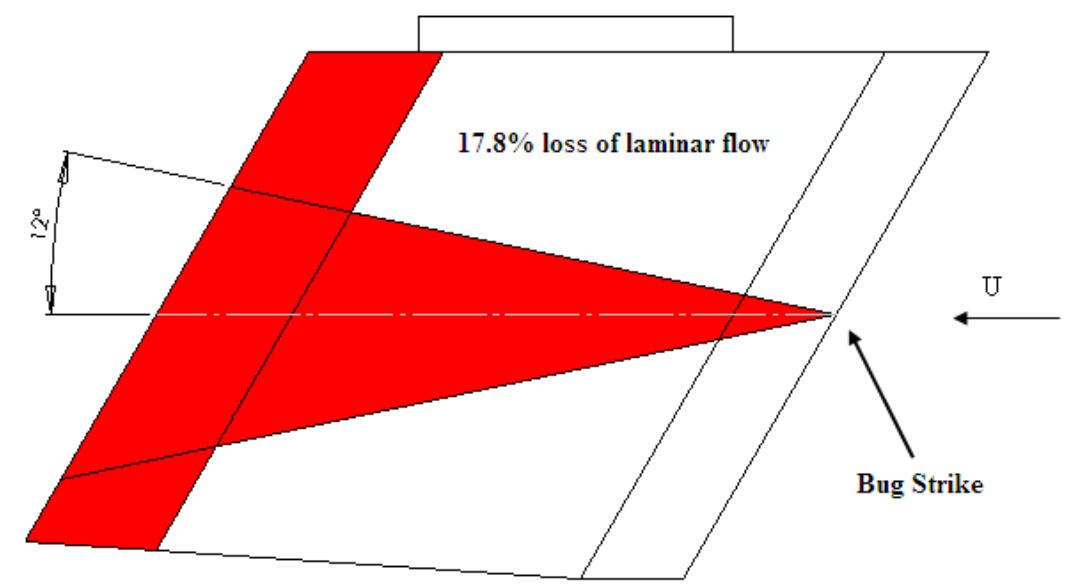

Figure 92: Percentage loss of laminar flow due to a bug strike and assuming transition at $80 \% x / c$. 


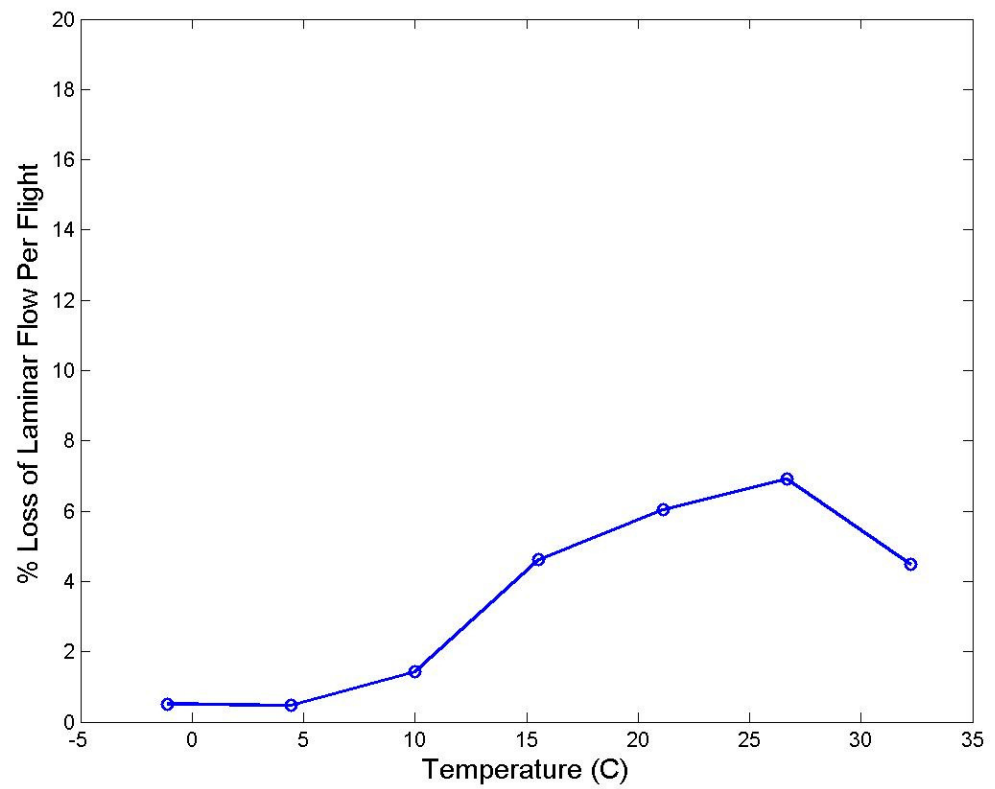

Figure 93: Percentage loss of laminar flow on the SWIFT model as a function of ground temperature.

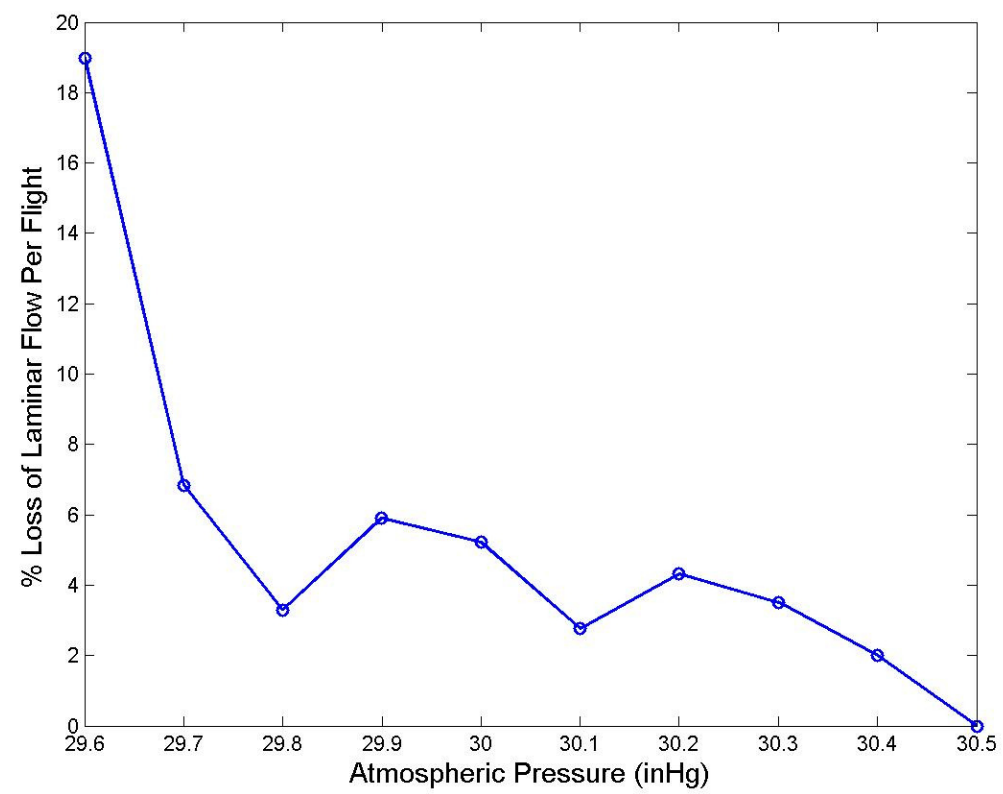

Figure 94: Percentage loss of laminar flow on the SWIFT model as a function of atmospheric pressure. 


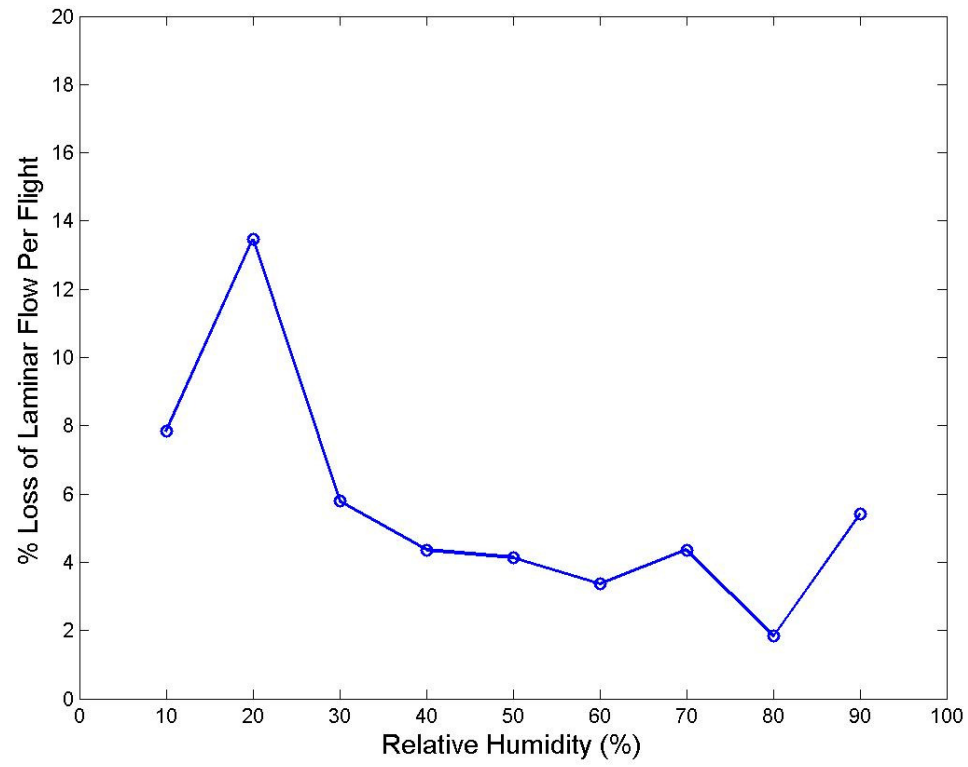

Figure 95: Percentage loss of laminar flow on the SWIFT model as a function of relative humidity.

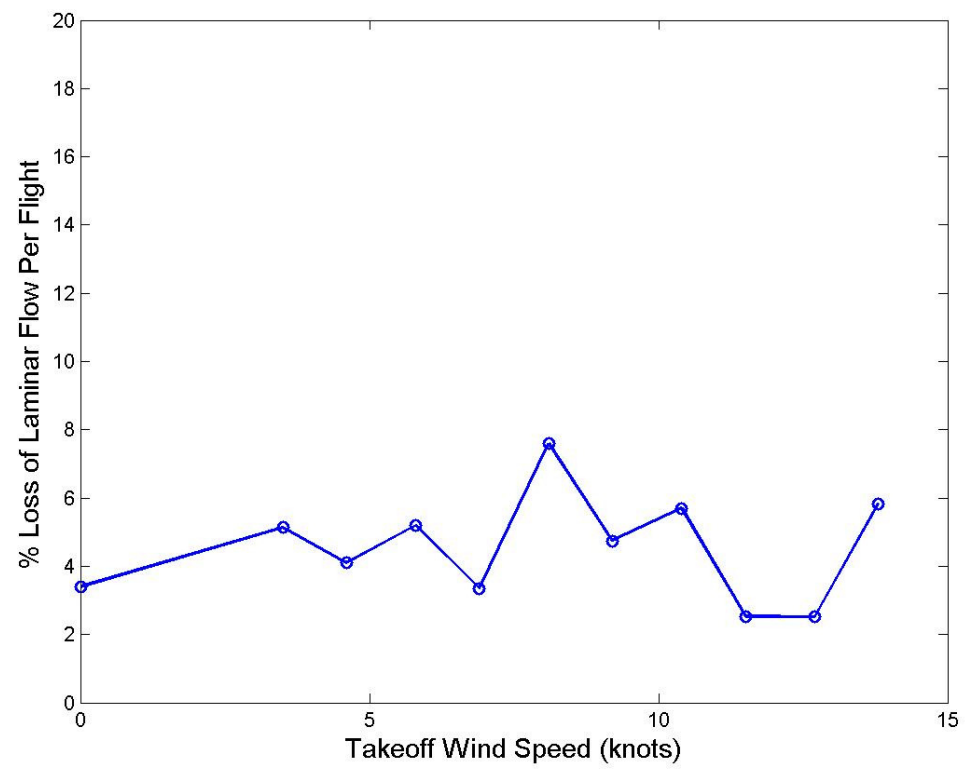

Figure 96: Percentage loss of laminar flow on the SWIFT model as a function of wind speed. 
In some cases the percentage loss of laminar flow is significant. Flights conducted with ground temperatures at $25{ }^{\circ} \mathrm{C}$ with ground winds around 8 knots are at the highest risk of insect contamination. In a flight-test environment, where the takeoff time can revolve around the weather, the risk associated with insect contamination can be mitigated by not flying during peak insect-activity times. Furthermore, the time at the lower altitudes are very short, reducing the possibility of bug strikes. An aircraft capable of 1000 feet per minute climb rates will only be exposed to a high density of bugs during the first 30 seconds of the flight. Lastly, these statistics were collected in Eastern Texas where bug population densities are high. Flight tests conducted in the desert are expected to show similar trends, but the amount of bug strikes and percentage loss of laminar flow is expected to be lower.

On the other hand, an operational airline with a laminar-flow wing will require an insect protection device. The amount of bugs collected during takeoff on any given flight may be low, but it is unlikely that the maintenance crew will wipe down the leading edge prior to the next flight, resulting in an unacceptable level of bug-remnant accumulation. The NASA Jetstar experiments showed two different techniques for protecting the leading edge from bug strikes (Maddalon \& Braslow 1990). Both designs showed very promising results in simulated airline service and can be incorporated into the design of future laminar-flow wings. 


\section{CONCLUSIONS}

A flight-test platform has been created for studying boundary layer transition on swept-wings. The Cessna O-2A aircraft is capable of carrying large external stores and has plenty of cabin space and electrical power available for large instrumentation packages. Although there are substantial changes in the static stability of the aircraft with the SWIFT model, the dynamic stability changes little with the model. As a result, the Cessna O-2A is a safe and valuable aircraft for conducting such flight tests.

Freestream disturbance levels underneath the outboard, port pylon location have been measured to be $0.05 \%$ to $0.07 \%$ of the freestream velocity. Freestream conditions have been calculated and verified experimentally to differ between the port wing and starboard wing. As a result, all freestream measurements must be conducted locally around the test article of interest for these tests and all future experiments.

$C p$ measurements on the SWIFT model have demonstrated large spanwise variations between the two pressure port rows. However, good agreement has been achieved between experimental $C p$ measurements and computations, providing confidence that the mean-flow calculations in the CFD are correct. The short span of the SWIFT model, combined with end effects have resulted in a useable test area of approximately 16 inches in between the pressure port rows. This space is sufficient span to conduct these experiments. 
Large amounts of NLF have been recorded on this model with both a polished and painted leading edge. This fact confirms the low freestream turbulence measurements and the general assumption that flight is a low-disturbance environment. At AoAs greater than -3.5 degrees the model generates significant NLF and transition is independent of leading edge roughness. It is assumed that transition is T-S dominated at AoAs greater than -3.5 degrees. Below -3.5 degrees AoA, the model is crossflow dominated because a high sensitivity to leading edge roughness is observed. Furthermore a characteristic saw-tooth pattern, typical of a crossflow instability, starts to emerge in the IR images.

Two entirely different behaviors were recorded with critically-spaced roughness. The appliqué DREs with cylindrical cross sections tripped the boundary layer at heights calculated to be subcritical. No change in transition was observed with the variableheight DREs, despite pressurizing the system to roughness heights more than 2.5 times those tested with the appliqué DREs.

A new technique of calibrating hotfilms with a Preston tube was developed. A simple algebraic equation was created for taking into account temperature compensation over large temperature excursions. This equation was also used to account for the heatconduction loss in the model, which has historically been the most difficult parameter to account for when calibrating surface-mounted hotfilms. After calibrating the hotfilm, disturbance amplitudes were measured downstream of modulating roughness heights and 
appliqué DREs. There appears to be no significant difference between the disturbances generated by an appliqué DRE versus a variable-height DRE, confirming the idea that the applique DREs were tripping the boundary layer in some of the early tests. Additionally, hotfilm measurements have shown that little crossflow growth occurs at the -2.61 degrees $A o A$ condition. However, strong crossflow growth occurs at -4.69 degrees AoA, and a difference in disturbance amplitudes is observed as roughness height is increased. These data can now be used to correlate roughness height to initial disturbance-amplitude inputs in the NPSE calculations. However, additional measurements need to be made in the linear growth regime in order to complete the receptivity picture.

Swept-Wing Laminar Flow Control was demonstrated with 12- $\mu \mathrm{m}$ high roughness elements on a painted leading edge. Despite demonstrating the importance of having a uniform base to apply DREs, no results were obtained with the polished leading edge. However, a 100\% increase in laminar flow was achieved for one of the test points with the painted leading edge.

Lastly, a comparison between the bug strikes collected on these flights and the literature has been made. There is good agreement between the insect population density as functions of wind speed, temperature, and altitude. Furthermore, a correlation between the number of bug strikes on a straight wing versus a swept-wing was made. In these experiments the swept-wing picked up 33\% less bug strikes compared to the 
straight wing. Lastly, recommendations were made for operating a laminar-flow wing in both a flight-test environment and an operational airline. 


\section{REFERENCES}

Agarwal N.K., Maddalon D.V., Mangalam S.M. \& Collier F.S. 1992 Crossflow vortex and transition measurements by use of multi-element hotfilms. AIAA J. 30(9):2212-18.

ARnAL D. 1994 Predictions based on linear theory. Progress in transition modeling, AGARD Rep. No. 709.

Banks D.W., VAn Dam C.P., Shiu H.J. \& Miller G.M. 2000 Visualization of in-flight flow phenomena using infrared thermography. NASA TM 209027.

BECHERT D.W. 1995 Calibration of preston tubes. AIAA J. 34(1):205-6.

Bellhouse B.J. \& SchulTz DL. 1966 Determination of mean and dynamic skin friction, separation and transition in low-speed flow with a thin-film heated element. J. Fluid Mech. 24:379-400.

BIPPES H. 1997 Environmental conditions and transition prediction in 3-D boundary layers. AIAA Paper 97-1906.

Brandon J.M., Manuel G.S., Wright R.E. \& Holmes BJ. 1990 In-flight flow visualization using infrared imaging. Journal of Aircraft 27:612-8.

CARPENTER A.L. 2009 In-Flight Receptivity Experiments on a 30-Degree Swept-Wing using Micron-Sized Discrete Roughness Elements. AIAA Paper 2009-590.

CARMichael B.H. 1958 Critical Reynolds numbers for multiple three-dimensional roughness elements. Northrop Aircraft, Inc. Report No. N.A.I.-58-589(BLC-112).

CHAPMAN K.L. 1996 Structure identification within a transitioning swept-wing boundary layer. PhD thesis, Clarkson University.

Chapman K., Reibert M., Saric W. \& Glauser M. 1998 Boundary-layer transition detection and structure identification through surface shear stress measurements. AIAA Paper 98-0782.

Choudhari M. \& STREetT C. 1990 Boundary layer receptivity phenomena in threedimensional and high-speed boundary layers. AIAA Paper 90-5258.

Coleman W.S. 1961 Roughness due to insects. In Boundary Layer and Flow Control, Vol. 2 (Ed. G.V. Lachmann) Pergammon Press, pp. 682-747.

COLLIER F.S. Jr. 1993. An overview of recent subsonic laminar flow control flight experiments. AIAA Paper 93-2987.

Croom C.C. \& Holmes B.J. 1986 Flight evaluation of an insect contamination protection system for laminar flow wings. SAE Paper 850860. 4486-94.

Dagenhart J.R., Saric W.S., Mousseux M.C. \& Stack JP. 1989 Crossflow-vortex instability and transition on a 45-degree swept wing. AIAA Paper 89-1892. 
DAVIS A.L. 1968 Model O-2 pylon fatigue test. Cessna Aircraft Company, Model M337, Report No. S-M337-45.

DEYHLE H. \& BIPPES H. 1996 Disturbance growth in an unstable three-dimensional boundary layer and its dependence on initial conditions. J. Fluid Mech. 316:73-113.

Fischer M.C., Wright A.S. JR \& WAGNER R.D. 1983 A flight test of laminar flow control leading-edge systems. NASA TM 85712.

Fowell L.R. \& Antonatos P.P. 1965 Some results from the X-21A program. Part 2. Laminar flow flight test results on the X-21A. AGAR-Dograph 97.

GREEN J.E. 2008 Laminar flow control - back to the future? AIAA Paper 2008-3738.

HAYNES T.S. \& REED H.L. 2000 Simulation of swept-wing vortices using nonlinear parabolized stability equations. J. Fluid Mech. 405:325-49.

Herbert T. 1997 Parabolized stability equations. J. Fluid Mech. 29:245-83.

MACK LM. 1984 Boundary-layer linear stability theory. Spec. Course Stab. Transit. Laminar Flows, AGARD Rep. 709, pp. 3.1-81.

MADDALON M.D. \& BRASLOW A.L. 1990 Simulated-airline-service flight tests of laminar-flow control with perforated-surface suction system. NASA TP 2966.

Mangalam S.M., Maddalon D.V., SARIC W.S. \& Agarwal N.K. 1990 Measurement of crossflow vortices, attachment-line flow, and transition using microthin hotfilms. AIAA Paper 90-1636.

Martin M.L., Carpenter A.L. \& SARIC W.S. 2008. Swept-wing laminar flow control studies using Cessna O-2A test aircraft. AIAA Paper 2008-1636.

Martinez J.R., Flick P., Perdzock J. \& Dale G. 2008 An overview of sensorcraft capabilities and key enabling technologies. AIAA Paper 2008-7185.

MCKNIGHT C.W. 2006 Design and safety analysis of an in-flight, test airfoil. MS thesis, Texas A\&M University.

Miley S.J., VAn Dam C.P., YiP L.P., Willard P.E., Crowder J.P. \& WaZlavick R.L. 1997 Slat transition characteristics on the NASA B737-100 aircraft using infrared imaging and hot-film anemometry. In Flow Visualization, Vol VII (Ed. J.P. Crowder) Begell House, New York, pp. 950-6.

Moes T.R., Sarma G.R., Mangalam S.M. 1997 Flight demonstration of a shock location sensor using constant voltage hotfilm anemometry. NASA TM 4806.

PATE C. 1967. Vibration and flutter analysis. Cessna Aircraft Company, Model M337, Report No. S-M337-27-3.

PATEL V.C. 1965 Calibration of the preston tube and limitations on its use in pressure gradients. J. Fluid Mech. 35:185-208. 
PETERSON J.B. JR \& FISHER D.F. 1978 Flight investigation of insect contamination and its alleviation. NASA CP 2036.

PRESTON J.H. 1954. The determination of turbulent skin friction by means of pitot tubes. J. Roy. Aero. Soc. 58:109-21.

RADEZTSKY R.H. JR, REIBERT M.S., SARIC W.S. \& TAKAGI S. 1993a Effect of micronsized roughness on transition in swept-wing flows. AIAA Paper 93-0076.

RADEZTSKY R.H., REIBERT M.S. \& TAKAGI S. 1993b A software solution to temperature-induced hot-wire voltage drift. In Proc. of the Third International Symposium on Thermal Anemometry.

REED H.L. \& SARIC W.S. 1989 Stabilty of three-dimensional boundary layers. Annu. Rev. Fluid Mech. 21, 235-284.

REED H.L. \& SARIC W.S. 2008 Transition mechanisms for transport aircraft. AIAA Paper 2008-3743.

ReIBert M.S., SARIC W.S., CARrillo R.B. JR \& ChAPMAN K.L. 1996 Experiments in nonlinear saturation of stationary crossflow vortices in a swept-wing boundary layer. AIAA Paper 96-0184.

RESHOTKO E. 1994 Boundary-layer instability, transition and control. AIAA Paper 940001.

Rhodes R.G., CARPenter A.L., ReEd H.L. \& SARIC W.S. 2008 CFD analysis of flighttest configuration for LFC on swept wings. AIAA Paper 2008-7336.

Runyan L.J., Bielak G.W., Rehbahani R., CHEN A.W. \& RozendaAl R. 1987757 NLF glove flight test results. NASA CP 2487, pp. 795-818.

RYDMAN A.G. \& PALMER R.E. JR. 1969 Category II performance and qualitative stability and control tests of the O-2A aircraft. Air Force Flight Test Center, Technical Report No. pp. 68-15.

SARIC W.S. 2007 Boundary-layer stability and transition. In Springer Handbook of Experimental Fluid Mechanics (Ed. Cameron Tropea, Alexander Yarin, John F. Foss) Springer-Verlag Berlin Heidelberg, pp. 886-896.

Saric W.S., Carpenter A.L., Hunt L.E., McKnight C.W. \& Schouten S.M. 2006 SWIFT - safety analysis for swept-wing experiments. TAMUS-AE-TR-06-002, Technical Report.

SARIC W.S., CARriLlo R.B. JR, REIBERT M.S. 1998 Leading-edge roughness as a transition control mechanism. AIAA Paper 98-0781.

SARIC W.S. \& REED H.L. 2002 Supersonic laminar flow control on swept wings using distributed roughness. AIAA Paper 2002-0147.

SARIC W.S., REED H.L. \& BANKS D.W. 2005 Flight testing of laminar flow control in high-speed boundary layers. NATO-RTO-MP-AVT-111/RSM. 
SARIC W.S., REED H.L. \& KERSCHEN E.J. 2002 Boundary-layer receptivity to freestream disturbances. Annu. Rev. Fluid Mech. 34:291-319.

SARIC W.S., ReED H.L. \& WhITE E.B. 2003 Stability and transition of threedimensional boundary layers. Annu. Rev. Fluid Mech. 35:413-40.

SARMA G.R. \& MoES TR. 2005 Demonstration of skin friction measurements featuring in situ estimation of conduction loss using constant voltage anemometers and surface hotfilms. Rev. Sci. Instruments 76:055102.1-.6

SMITH A.M.O. 1956 Transition, pressure gradient, and stability theory. Proc. 9th Int. Congress of App. Mech., Brussels, 4:234-44.

SMith A.M.O \& GAMBERONi N. 1956 Transition, pressure gradient, and stability theory. Douglas Aircraft Co. Rep. ES-26388.

U.S. Standard Atmosphere. 1976 Government Printing Office, Washington, D.C.

VAN Dyke L.P. 1970 M337 pylon test. Cessna Aircraft Company, Model M337, Report No. S-000-195.

VAN INGEN J.L. 1956 A suggested semi-empirical method for the calculation of the boundary layer transition region. Univ. Delft Rep. VTH-74.

Von Doenhoff A.E. \& BRAslow A.L. 1961 The effect of distributed surface roughness on laminar flow. Boundary Layer Control. Lachmann V (ed). Pergamon, Oxford, 2:657-81.

White E.B. 2000 Breakdown of crossflow vortices. PhD thesis, Arizona State University.

WHITE E.B. \& SARIC WS. 2000 Application of variable leading-edge roughness for transition control on swept wings. AIAA Paper 2000-0283.

WhIte E.B., SARIC W.S. 2005 Secondary instability of crossflow vortices. J. Fluid Mech. 525:275-308.

WoAn C.J., Gingrich P.B. \& GEORGE M.W. 1991 CFD validation of a supersonic laminar flow control concept. AIAA Paper 91-0188.

ZUCCHER S. \& SARIC W.S. 2007 Infrared thermography investigations in transitional supersonic boundary layers. Experiments in Fluids. 44:145-57. 


\section{VITA}

Name: $\quad$ Andrew Lee Carpenter

Address: $\quad$ Texas A\&M University, Department of Aerospace Engineering H.R. Bright Building, Rm. 701, TAMU 3141

College Station TX 77843-3141

Email Address: andrewleecarpenter@gmail.com

Education: $\quad$ B.S., Aerospace Engineering, Arizona State University, 2003

Ph.D., Aerospace Engineering, Texas A\&M University, 2009 